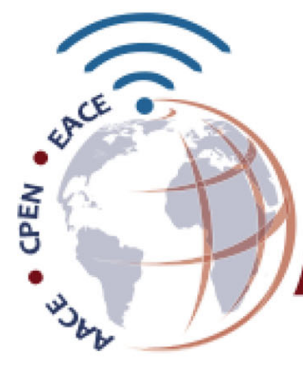

A VIRTUAL EDUCATION EVENT

\title{
2020 International Cancer Education Conference Program and Abstracts
}

\section{Program Co-Chairs:}

Clement Gwede PhD, MPH, RN, FAAN Jila Tanha MPH, CHES

\section{Program Vice-Chairs:}

Faith Addiss RN

Jill Hamilton PhD, RN, FAAN
Jointly Provided By:

Amedco LLC and the American

Association for Cancer Education
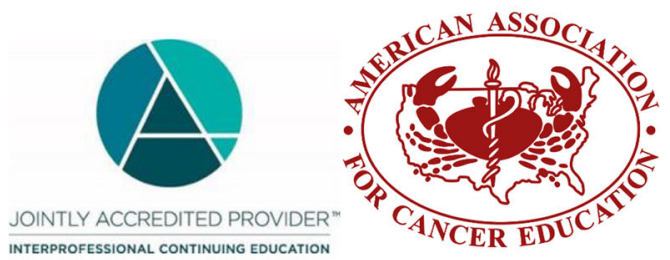

Jointly Organized by:
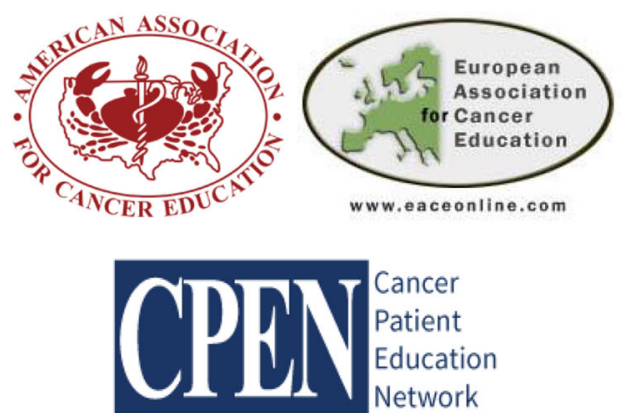


\title{
2020 International Cancer Education Conference Program and Abstracts
}

\author{
Published online: 26 February 2021 \\ (C) American Association for Cancer Education 2021
}

The American Association for Cancer Education (AACE), the Cancer Patient Education Network (CPEN), and the European Association for Cancer Education (EACE), in conjunction with our co-provider, the University of South Florida (USF) Health, will sponsor the premier meeting in North America dedicated to cancer education, the International Cancer Education Conference (ICEC). The meeting is designed to support cancer educators to learn best practices for cancer prevention, diagnosis, treatment and survivorship. Our overarching goal is to increase positive outcomes for cancer patients/survivors and their families and the general public. Effective evidence-based education is pivotal in developing cancer scientists, advancing cancer curriculum and therapies in professional health education programs and in achieving optimal outcomes for cancer patients/survivors. The use of best practices in professional, patient and family, and public education contributes significantly to the ongoing national and international efforts to reduce cancer morbidity and mortality.

The goal of the ICEC is to facilitate interdisciplinary research and practice/program collaborations among cancer researchers and educators on a national and global level. Educational models, programs and research strategies will be presented to support the development of evidence-based practices in the field of cancer education.

\section{NEEDS ASSESSMENT}

Nearly $39 \%$ of the US population will be diagnosed with cancer in their lifetime, and the number of people living beyond a cancer diagnosis is expected to rise to almost 19 million by 2024 (NCI, 2017). Beyond the US, cancer remains one of the leading causes of death in the world (WHO, 2018).

Action and education of providers and adoption of evidence-based practices are two key methods to accelerate improvements in cancer outcomes (Spinks et al., 2012). In order to support reductions in cancer death rates and focus attention on reducing cancer-related health disparities (including prevention, early detection, and treatment of less common cancers), the professional workforce must be educated and well-informed about cutting-edge research and evidence-based practices. A key challenge in the oncology field is timely dissemination and implementation of scientific discoveries/new treatments in clinical practice and training programs. Cancer educators play a crucial role in the global setting to increase the quality of cancer care and positive outcomes for clinicians, cancer patients/survivors and their families.

\section{Clinical Practice Learning Gaps}

This conference will address the following learning gaps among cancer education professionals, and students:

Up-to-date information on evidence-based practices related to alleviating disparities, cancer diagnosis, treatment and survivorship care.

New knowledge on innovative delivery models (including information technology, mobile technology, telehealth, and social media) to address the education, information, and resource needs of patients/survivors and families. Evidence shows that people are using technology to access care and treatment information (Pew Research Center, 2014).

Skills related to health literacy, communication, forming community partnerships, and psychosocial education strategies with a specific focus on community outreach to special populations.

How genomics are integrated into decision making across the cancer continuum from prevention, screening/early detection, diagnosis, treatment, survivorship and beyond.

\section{LEARNING OBJECTIVES}

The International Cancer Education Conference explores new and creative education models that support best practice developments in the field and facilitate interdisciplinary research across the cancer care continuum. This year's conference will have an extensive focus on the use of effective and innovative formats and technologies; genetics, genomics, and precision medicine; disparities; special populations; global cancer education initiatives; and advocacy.

After attending this conference, the participant shall be able to:

Healthcare Professional Education

- Develop strategies that explore and address social determinants of health to alleviate cancer disparities.

- Identify and support training, interdisciplinary collaboration, and mentorship opportunities for junior investigators and practitioners to support their development as cancer researchers and educators.

- Plan and advocate for interdisciplinary programs that promote patient-centered care and equity in diagnosis, prevention, treatment, and survivorship of cancer for patients, their families, communities, and society.

Patient, Family, and Public Education

- Identify and implement innovative and evidence-based cancer education strategies, including culturally, linguistically, and literacy relevant approaches.

- Discuss creative and effective approaches to incorporating multimedia and technology in cancer education, including in the design, implementation, and evaluation of programs.

- Promote best practices of patient-centered care to improve social, economic and environmental conditions, promote health equity and engage and educate patients, families, and survivors.

- Discuss new technologies in genetics, genomics, and precision medicine across the continuum of cancer care (from prevention to survivorship).

Global Outreach

- Enhance collaborations to enable participants across the care continuum to foster new discoveries and lead to lowering the global burden of cancer.

- Identify opportunities to support and promote biomedical, clinical, behavioral, and health services research that is culturally competent and responsive to the needs of underserved populations.

- Support activities that establish and promote global cancer education initiatives among national and international cancer education organizations. 


\section{TARGET AUDIENCE}

Oncologists, primary care physicians, surgeons, radiologists, ministries of health, cancer experts (government and association), researchers, nurses, dentists, geneticists, epidemiologists, behavioral scientists, pharmacists, pharmacologists, health educators, librarians, social workers, allied and public health professionals, students, and patient advocacy groups will be in attendance.

Past participants have included attendees from the world's medical centers, cancer care organizations, and academic institutions responsible for developing, implementing, and evaluating cancer education curricula, research, and programs.

\section{CONFERENCE GOALS}

ICEC attendees will engage in professional development through educational opportunities, including:

- Expert plenary sessions and abstract presentations addressing topics of disparities in cancer care and research, precision medicine, genomics, survivorship, professional education (medical, nursing, and allied health) and global innovations in cancer education.

- Skill-based workshops addressing cancer education including grant writing; publishing; utilizing a translator or interpreter; survivorship; health literacy; working with underserved populations; genetics and cancer; palliative care; and more.

- Poster presentations featuring over 100 research studies, evidencebased practices in oncology, innovative education programs and quality improvement projects.

- Forums focused on ongoing multi-institutional programs on current topics in cancer education including targeted therapies, survivorship, health literacy, social media, professional training and international outreach with disparate populations.

- Networking opportunities and scheduled mentorship with international leaders and researchers in cancer education to enable collaborations that address common cancer education challenges and share new and existing resources.

\section{ACCREDITATIONS}

In support of improving patient care, this activity has been planned and implemented by Amedco LLC and the American Association for Cancer Education. Amedco LLC is jointly accredited by the Accreditation Council for Continuing Medical Education (ACCME), Accreditation Council for Pharmacy Education (ACPE), and the American Nurses Credentialing Center (ANCC), to provide continuing education for the healthcare team.

\section{Physicians:}

Amedco LLC designates this live and enduring material for a maximum of 21.00 AMA PRA Category 1 CreditsTM. Physicians should claim only the credit commensurate with the extent of their participation in the activity.
Nurses:

Amedco LLC designates this activity for a maximum of 21.00 ANCC contact hours.

Certified Health Education Specialists (CHES):

This program is designated for Certified Health Education Specialists (CHES) and/or Master Certified Health Education Specialists (MCHES) to receive up to 21.00 total Category I continuing education contact hours. Maximum advanced-level $\mathrm{CECH}$ available are 21.00. Advanced-level contact hours earned Provider ID \# 98001. Program number is 36711 .

Social Workers (application by AACE alone, not jointly with Amedco) This program is Approved by the National Association of Social Workers (Approval \# 886679014-2245) for 22 continuing education contact hours.

\section{DISCLOSURES}

Disclosure of Relevant Financial Relationships with Commercial Interests

Amedco endorses the standards of the ACCME and ANCC that require everyone in a position to control the content of accredited educational activity to disclose all financial relationships with commercial interests that are related to the content of the educational activity. All accredited activities must be balanced, independent of commercial bias and promote improvements or quality in healthcare. All recommendations involving clinical medicine must be based on evidence accepted within the medical profession.

A conflict of interest is created when individuals in a position to control the content of an accredited educational activity have a relevant financial relationship with a commercial interest which therefore may bias his/her opinion and teaching. This may include receiving a salary, royalty, intellectual property rights, consulting fee, honoraria, stocks or other financial benefits.

Amedco will identify, review and resolve all conflicts of interest that speakers, authors or planners disclose prior to an educational activity being delivered to learners. Disclosure of a relationship is not intended to suggest or condone bias in any presentation but is made to provide participants with information that might be of potential importance to their evaluation of a presentation. Amedco does not endorse any products or services.

The following speakers, authors and planners have provided Amedco with disclosures of relevant financial relationships that exist and may be considered a potential conflict of interest. Presentations of these individuals have been peer-reviewed and were found to be balanced, to be free of commercial bias, and to promote improvements or quality in healthcare:

Presenter: Nicole Stout, West Virginia Cancer Institute

Potential Conflict: Dr. Stout provides educational lectures for Medbridge Inc.

All other speakers, authors and planners have disclosed no potential conflicts of interest as of 30 September 2020. 


\section{EXECUTIVE PLANNING COMMITTEE}

*Faith Addiss RN, Roswell Park Cancer Institute

Beth Bartholomew, St. Jude Children's

Research Hospital

Chesley Cheatham MEd, MCHES, University Hospitals Seidman Cancer Center

Katie Cueva ScD, MAT, MPH, Institute of Social and Economic Research, University of Alaska

${ }^{*}$ Clement Gwede PhD, MPH, RN, FAAN, H. Lee Moffitt Cancer Center \& Research Institute

*Jill Hamilton PhD, RN, FAAN, Emory University

Bret Hassel PhD, University of Maryland School of Medicine

*Conference Nursing Content Expert and Reviewer
John Luque PhD, MPH, Florida A\&M University

James Marmion, St. Jude Children's Research

Hospital

Liliana Mulato, Huntsman Cancer Institute

Siddhartha Roy DrPH, MPH, Pennsylvania State University

Renee Siegel MSW, LCSW, Dana-Farber Cancer Institute

Jila Tanha MPH, CHES, MD Anderson Cancer Center

\begin{tabular}{l} 
Abstracts Subcommittee \\
\hline Co-Chair: Juan Luque \\
Co-Chair: Liliana Mulato \\
Ana Barros \\
Debbie Cadet \\
Joycelyn Cudjoe \\
Stacy Davis \\
Clement Gwede \\
Meg McCallum \\
Devon Poznanski \\
Saima Saleem \\
Bethany Sanborn \\
Kay See Tan \\
Cheri Tolle \\
Pamela Valera \\
Chasity Walters \\
Gerard Zambetti
\end{tabular}

\section{Fundraising and Marketing Subcommittee Co-Chair: Katie Cueva Co-Chair: Renee Siegel Jerone Farley Clement Gwede Bret Hassel}

\section{Roundtables Subcommittee}

Co-Chair: Faith Addiss

Co-Chair: James Marmion

Angela Adjetey Appiah

Chesley Cheatham

Louise Cunningham-Rambo

Aldenise Ewing

Carolyn Messner

Maureen Rigney

Lisa Wigfall

Michelle Williams

\author{
Poster Judging \\ Subcommittee \\ Co-Chair: Siddhartha Roy \\ Co-Chair: Jila Tanha \\ Angela Adjetey Appiah \\ Aldenise Ewing \\ Maria Jibaja-Weiss \\ Carolyn Messner \\ Maureen Rigney \\ Lisa Wigfall \\ Michelle Williams
}

Local Subcommittee

Chair: Bret Hassel 


\section{CONFERENCE PROGRAM}

\begin{tabular}{|c|c|c|c|}
\hline \multirow{6}{*}{ 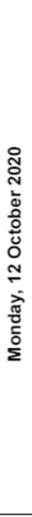 } & $\begin{array}{l}11: 00 \mathrm{AM}- \\
\text { 12:30 PM } \\
\text { EDT }\end{array}$ & $\begin{array}{c}\text { WORKSHOP } \\
\text { Essential Skills in Cancer Education: Leadership, Leading and Influencing Change in Cancer Education - } \\
\text { A Comprehensive Interactive Hands-On Workshop } \\
\text { Ewa Szumacher (Sunnybrook Health Sciences Centre): Krista Dawdy (Odette Cancer Centre); } \\
\text { Maria Bishop (University of A Aizona College of Medicine); Gilad Amiel (RAMBAM Healthcare Campus); } \\
\text { David Wijer (University Health Network); Kathleen Heneghan (American College of Surgeons); } \\
\text { Jamal Khadar (King Hussein Cancer Centre) }\end{array}$ & $\begin{array}{l}\text { WORKSHOP } \\
\text { Best practices in the development of web-enabled cancer patient education tools for improved } \\
\text { decision making } \\
\text { Sarah Bass (Temple University); Linda Fleisher (Fox Chase Cancer Center); } \\
\text { Cassidy Kenny (Fox Chase Cancer Center) }\end{array}$ \\
\hline & $\begin{array}{c}12: 30-1: 30 \\
\text { PM EDT }\end{array}$ & \multicolumn{2}{|c|}{ BREAK (visit posters/exhibits) } \\
\hline & $\begin{array}{l}\text { 1:30-3:00 } \\
\text { PM EDT }\end{array}$ & $\begin{array}{c}\text { WORKSHOP, continued } \\
\begin{array}{c}\text { Essential Skills in Cancer Education: Leadership, Leading and Influencing Change in Cancer Education - } \\
\text { A Comprehensive Interactive Hands-On Workshop }\end{array}\end{array}$ & $\begin{array}{c}\text { WORKSHOP } \\
\begin{array}{c}\text { Advancing Antiracist Psychosocial Care to Address Financial Hardship in Adolescent \& Young Adult } \\
\text { Oncology }\end{array} \\
\begin{array}{c}\text { Christabel K. Cheung (University of Maryland School of Social Work and University of Maryland } \\
\text { Greenebaum Comprehensive Cancer Center) }\end{array}\end{array}$ \\
\hline & $\begin{array}{l}\text { 3:00-3:30 } \\
\text { PM EDT }\end{array}$ & \multicolumn{2}{|c|}{ BREAK (visit posters/exhibits) } \\
\hline & $\begin{array}{l}\text { 3:30-5:00 } \\
\text { PM EDT }\end{array}$ & \multicolumn{2}{|c|}{$\begin{array}{l}\text { Cancer Education in the Age of COVID-19: Disparities, Pandemics, and Infodemics } \\
\text { Monica Webb Hooper, National Institute on Minority Health and Health Disparities }\end{array}$} \\
\hline & $\begin{array}{l}\text { 5:00-5:30 } \\
\text { PMEDT }\end{array}$ & \multicolumn{2}{|c|}{ AACE New Member Meet-and-Greet } \\
\hline
\end{tabular}

\begin{tabular}{|c|c|c|c|c|c|}
\hline \multirow{8}{*}{ 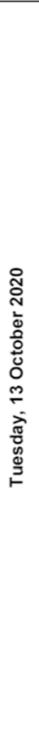 } & $\begin{array}{c}10: 00-11: 00 \\
\text { AM EDT }\end{array}$ & \multicolumn{2}{|l|}{ No program } & \multicolumn{2}{|c|}{$\begin{array}{ll}\text { WORKSHOP } \\
\end{array}$} \\
\hline & \multirow[t]{3}{*}{$\begin{array}{c}\text { 11:00 AM - } \\
\text { 1:00 PM EDT }\end{array}$} & \multirow{3}{*}{\multicolumn{2}{|c|}{$\begin{array}{c}\text { NCI TRAINING WORKSHOP } \\
\text { NCl's Support for Cancer Education Across the Academic Lifespan: R25 and UE5 Programs } \\
\text { - NCl supported R25 Cancer Research Education Grants Program (Radaev) } \\
\text { - Enhancing Diversity through Early Intervention: the NCI R25 Youth Enjoy Science (YES) Research Education } \\
\text { Program (Lin) } \\
\text { - NCI Awardee Skills Development Consortium (NASDC): Helping NCI-Funded Junior Faculty Succeed in } \\
\text { Academic Cancer Research Careers (Korczak) } \\
\text { Jeannette Korczack (National Cancer Institute), Sergey Radaev (National Cancer Institute), } \\
\text { Alison Lin (National Cancer Institute) }\end{array}$}} & \multicolumn{2}{|c|}{$\begin{array}{l}\text { Difficult Conversations in Health Literate Care } \\
\text { (sponsored by Cancer Education at the Princess Margaret Cancer Centre) } \\
\text { akos (Princess Margaret Cancer Centre), Meredith Giuliani (Princess Margaret Cancer Centre), } \\
\text { ger (Princess Margaret Cancer Centre), Janet Papadakos (Princess Margaret Cancer Centre) }\end{array}$} \\
\hline & & & & \multicolumn{2}{|r|}{ 11:30 AM-12:30 PM: BREAK } \\
\hline & & & & \multirow{2}{*}{\multicolumn{2}{|c|}{$\begin{array}{c}\text { WORKSHOP, continued } \\
\text { Difficult Conversations in Health Literate Care } \\
\text { (sponsored by Cancer Education at the Princess Margaret Cancer Centre) }\end{array}$}} \\
\hline & \begin{tabular}{c|}
$1: 00-2: 00$ \\
PM EDT
\end{tabular} & \multicolumn{2}{|l|}{ BREAK (visit posters/exhibits) } & & \\
\hline & $\begin{array}{c}\text { 2:00-3:30 } \\
\text { PM EDT }\end{array}$ & 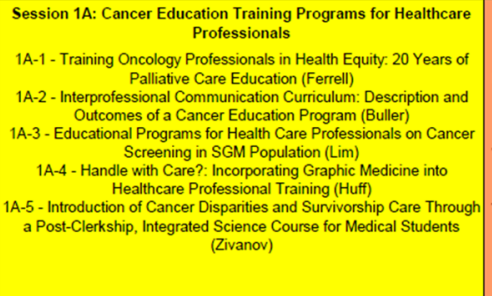 & \multicolumn{2}{|c|}{$\begin{array}{c}\text { Session 1B: Determinants of Cancer Prevention and Control } \\
\text { Behaviors } \\
\text { 18-1 - Using Culturally-Focused Stortelling to Empower Appalachian } \\
\text { Kentucky Youth to Understand and Address Cancer Disparties in Their } \\
\text { Communities (Nanderford) } \\
\text { 1B-2- Focus Group Discussions Among African Americans on } \\
\text { Colorectal Cancer Screening in the Southem US (Matthew) } \\
\text { 1B-3 - Application of the Integrated Behavior Model to non-theory-based } \\
\text { studies to identify determinants influencing catch-up HPV vaccination } \\
\text { outcomes (Olusanya) } \\
\text { 1B-4 - Social Determinants as Barriers to Mammography Screening at a } \\
\text { Community Cancer Screening Clinic (Hooper) } \\
\text { 18-5 - Using Geolocation Analysis to ldentify Gaps in Gynecological } \\
\text { Supportive Care Services and Enable Targeted Educational Efforts in } \\
\text { Rural Settings (Bhatia) }\end{array}$} & 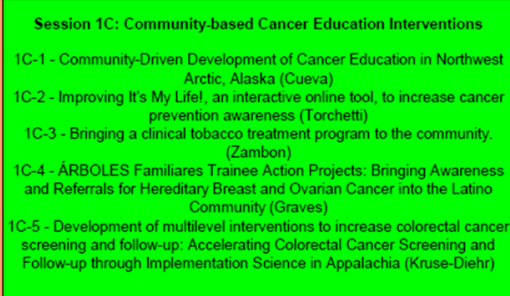 \\
\hline & $\begin{array}{c}3: 30-4: 00 \\
\text { PM EDT }\end{array}$ & \multicolumn{4}{|c|}{ BREAK (visit posters/exhibits) } \\
\hline & $\begin{array}{l}\text { 4:00-5:00 } \\
\text { PM EDT }\end{array}$ & \multicolumn{4}{|c|}{$\begin{array}{l}\text { Learning Conversations - a window to a world of enhanced Cancer Education } \\
\text { Ivan Silver, Centre for Addiction \& Mental Health, University of Toronto }\end{array}$} \\
\hline
\end{tabular}

\begin{tabular}{|c|c|c|c|c|}
\hline \multirow{7}{*}{ 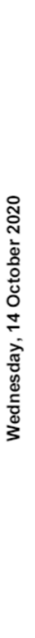 } & $\begin{array}{c}9: 30-10: 30 \\
\text { AM EDT }\end{array}$ & \multicolumn{3}{|c|}{ Roundtable Discussions (with breakouts in Zoom) } \\
\hline & $\begin{array}{c}\text { 10:30-11:00 } \\
\text { AM EDT }\end{array}$ & \multicolumn{3}{|c|}{ BREAK (visit posters/exhibits) } \\
\hline & $\begin{array}{l}11: 00 \text { AM - } \\
\text { 12:00 PM } \\
\text { EDT }\end{array}$ & & $\begin{array}{c}\text { PLENARY PRESENTATION } \\
\text { Diversity in cancer training - transforming challenges into opportuni } \\
\text { Alison Lin, National Cancer Institute }\end{array}$ & \\
\hline & $\begin{array}{c}12: 00-1: 30 \\
\text { PM EDT }\end{array}$ & & AACE Annual Business Meeting and Award Ceremony & \\
\hline & $\begin{array}{c}\text { 1:30 - } 3: 00 \\
\text { PM EDT }\end{array}$ & $\begin{array}{l}\text { Session 2A: Barriers to Cancer Care among Vulnerable } \\
\text { Populations } \\
\text { 2A-1 - A Literature Review of the Barriers to End-of-Life Care among } \\
\text { Hispanics with Advanced Cancer N Negative Connotations and Cost } \\
\text { Fears (Padilla) } \\
\text { 2A-2 - The Unique Challenges and Barriers to Cancer Treatment for } \\
\text { individuals with Autism: A review of the Literature and a Proposal for } \\
\text { Future Modifications (Cancino) } \\
\text { 2A-3 - Colorectal Cancer Risk Perception and Knowledge among } \\
\text { Caribbean University Students: A Cross-Sectional Study (Rocke) } \\
\text { 2A-4 - Acceptance of HPV Vaccination Myths Among Hispanics in a } \\
\text { US/Mexico Border Region: Literature review of Potential Interventions } \\
\text { (Chagolla) } \\
\text { 2A-5 - Primary care provider perceptions of multi-level barriers to } \\
\text { colorectal cancer screening and follow-up in Appalachian Kentucky } \\
\text { (Kruse-Diehr) }\end{array}$ & $\begin{array}{c}\text { Session 2B: Health Literacy Across the Cancer Control Continuum } \\
\text { 2B-1 - Difficult Conversations in Health Literate Care (Papadakos) } \\
\text { 2B-2 - Health Literacy Assessment of Cancer-Related Whiteboard } \\
\text { Animations for Patients (Quartey) } \\
\text { 2B-3- Evaluating the Feasibility and Utility of Machine Translation for } \\
\text { Patient Education Materials Written in Plain Language to Increase } \\
\text { Accessibility for Populations with Limited English Proficiency } \\
\text { (Umakanthan) } \\
\text { 2B-4 - COVID-19 \& Cancer Communication: An Evaluation of the } \\
\text { Literacy Demand of Consumer Health Resources (Reznikov) } \\
\text { 2B-5 - Establishing a framework for incorporating regular assessments } \\
\text { of Organizational Health Literacy in cancer centres across Ontario } \\
\text { (Giannopoulos) }\end{array}$ & $\begin{array}{c}\text { Session 2C: Using Technology to Expand the Reach of Cancer } \\
\text { Education to Healthcare Professionals } \\
\text { 2C-1 - Inuusinni Aqqusaaqtara: Using e-learning modules to engage Inuit } \\
\text { patients and healthcare providers (Torchetti) } \\
\text { 2C-2 - Educating Massage Therapists to Engage in Skin Cancer Primary } \\
\text { Prevention Conversations with Clients (Loescher) } \\
\text { 2C-3 - Princess Margaret Global Oncology Leadership Development } \\
\text { Program (Giuliani) } \\
\text { 2C-4 - The HPV Vaccine Project ECHO Program: A pilot study of a } \\
\text { telehealth program aimed at building clinician capacity to increase HPV } \\
\text { vaccine coverage among adolescents (Williams) } \\
\text { 2C-5 - Transition from In Person workshops to Online Learning for Patient } \\
\text { Navigation Training for Appalachia (Dignan) }\end{array}$ \\
\hline & \begin{tabular}{|c|}
$3: 00-3: 30$ \\
PM EDT \\
\end{tabular} & \multicolumn{3}{|c|}{ BREAK (with Wellness Activity, sponsored by University of Maryland Greenebaum Comprehensive Cancer Center) } \\
\hline & \begin{tabular}{|c|}
$3: 30-4: 30$ \\
PM EDT
\end{tabular} & \multicolumn{3}{|c|}{ Roundtable Discussions (with breakouts in Zoom) } \\
\hline
\end{tabular}




\section{CONFERENCE PROGRAM, continued}

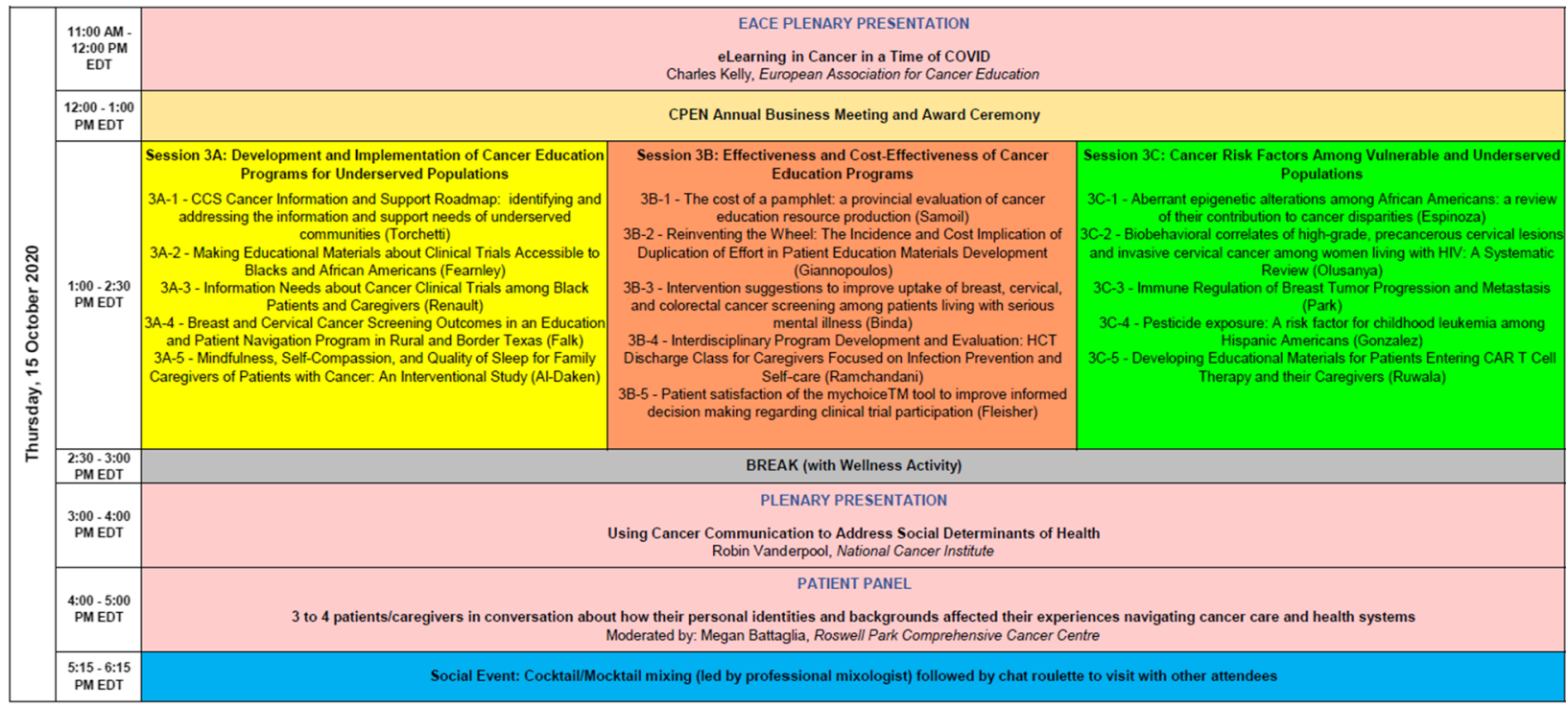

\begin{tabular}{|c|c|c|c|c|}
\hline \multirow{6}{*}{ 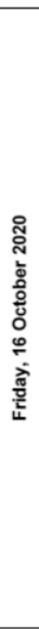 } & \multirow{2}{*}{\begin{tabular}{|c|}
$\begin{array}{c}11: 00 \mathrm{AM}- \\
12: 00 \mathrm{PM} \\
\text { EDT }\end{array}$ \\
$\begin{array}{c}12: 00-1: 00 \\
\text { PM EDT }\end{array}$ \\
\end{tabular}} & \multicolumn{3}{|c|}{$\begin{array}{c}\text { PLENARY PRESENTATION } \\
\text { Cancer Disparities and Treatment Equity: Removing Barriers to Cancer Care in our Community } \\
\text { Chris Lathan, Dana-Farber Cancer /nstitute }\end{array}$} \\
\hline & & \multicolumn{3}{|c|}{ BREAK (sponsored by POINT Biopharma) } \\
\hline & $\begin{array}{c}1: 00-2: 30 \\
\text { PM EDT }\end{array}$ & 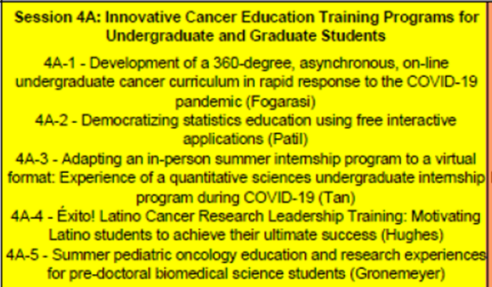 & $\begin{array}{c}\text { Session 4B: Late-Breaking Abstracts } \\
\text { 4B-1 - withdrawn } \\
\text { 4B-2 - Does Race and Medical Mistrust matter in Breast Cancer } \\
\text { Survivors' Health Beliefs? (Edmonds) } \\
\text { 4B-3 - Adult Cancer Survivorship eLeaming: Adapting high quality } \\
\text { nursing education for patients and families (Lawrie) } \\
\text { 4B-4 - Blending Cancer Prevention Science and Professional } \\
\text { Development: Communication Skills for Scientists-in-training (Monberg) } \\
\text { 4B-5 - Diamond Anniversary for the AACE (Hill) }\end{array}$ & $\begin{array}{l}\text { Session 4C: Cancer Education Programs for Patients and Caregivers } \\
\text { 4C-1 - Development and Testing of an Adjuvant Raciotherapy Decision Aid } \\
\text { for Older Women Diagnosed with Stage I Breast Cancer. A Pilot Study } \\
\text { (Szumacher) } \\
\text { 4C-2- Caregiver skills training programs in cancer care: a scoping review } \\
\text { (Samoil) } \\
\text { 4C-3 - An inner-city smoking cessation program for adults with low } \\
\text { socioeconomic status: a closer look at factors that predict successful } \\
\text { tobacco cessation (Levine) } \\
\text { 4C-4 - Group--Based Smoking Cessation Treatment Program for } \\
\text { Incarcerated Smokers (Valera) } \\
\text { 4C-5- Developing and Implementing an Effective New Cancer Patient } \\
\text { Resource (Huff) }\end{array}$ \\
\hline & $\begin{array}{c}2: 30-3: 00 \\
\text { PM EDT } \\
\end{array}$ & \multicolumn{3}{|c|}{ BREAK (with Wellness Activity) } \\
\hline & $\begin{array}{c}\text { 3:00 - } 4: 00 \\
\text { PM EDT }\end{array}$ & \multicolumn{3}{|c|}{$\begin{array}{l}\text { The Colors of CoviD-19: Confronting Health Disparities During a Global Pandemic } \\
\text { Stephen Thomas, Univeristy of Maryland School of Public Heaith }\end{array}$} \\
\hline & $\begin{array}{c}\text { 4:00 - 4:30 } \\
\text { PM EDT }\end{array}$ & \multicolumn{3}{|c|}{ CLOSING REMARKS, POSTER AWARDS, AND RAFFLE PRIZE } \\
\hline
\end{tabular}




\section{PRESENTING AUTHOR LIST}

Bidemi Adedokun (New Mexico State University)

P9

Laila Al-Daken (Sultan Qaboos University)

$3 \mathrm{~A}-5$

Gilad Amiel (Rambam Healthcare Campus)

Workshop 1

P37

$\mathrm{P} 23$

P21, P60

P49

Workshop 3

Plenary 6

1B-5

3B-3

Workshop 1

P48

1A-2

P55

$2 \mathrm{~A}-2$

$2 \mathrm{~A}-4$

P38

Workshop 4

Christabel Cheung (University of Maryland School of Social Work and University of Maryland Greenabaum Comprehensive Cancer Center)

Anthony Cirilo (University of California, San Diego)

P29

P44

P42

$1 \mathrm{C}-1$

P62

Workshop 1

$2 \mathrm{C}-5$

4B-2

$3 \mathrm{C}-1$

P16

P43

$3 \mathrm{~A}-4$

P56

$3 \mathrm{~A}-2$

1A-1

Workshop 3, 3B-5

4A-1

$\mathrm{P} 22$

P50

2B-5, 3B-2

Workshop 2, 2C-3

3C-4

4A-5

P30

P4

Workshop 1

4B-5

1B-4

1A-4, 4C-5

$4 \mathrm{~A}-4$ 
(continued)

Caitlyn Johnson (University of South Florida, Morsani College of Medicine)

Charles G. Kelly (Newcastle University)

Cassidy Kenny (Fox Chase Cancer Center)

Jamal Khader (King Hussein Cancer Centre)

Dina Koes (San Diego State University)

Jeanette Korczach (National Cancer Institute)

Dalia Koujah (University of California San Diego Moores Cancer Center)

Hatai Kraushaar (Sinai Hospital)

Aaron Kruse-Diehr (University of Kentucky)

Christopher S. Lathan (Dana-Farber/Harvard Cancer Center)

Karen Lawrie (Princess Margaret Cancer Centre)

Nicolette Olivia Le (University of California San Diego Moores Cancer Center)

Juan Andrew Leal, University of California San Diego)

Nicole Lee (University of California San Diego Moores Cancer Center)

Rachel Levine (Dana-Farber Cancer Institute)

Andrew Lim (University of Nebraska Medical Center)

Alison Lin (NCI Center to Reduce Cancer Health Disparities)

Nicole Liscio (Princess Margaret Cancer Centre)

Anni Liu (University of South Florida Morsani College of Medicine)

Jala Lockhart (The University of Alabama)

Lois Loescher (University of Arizona)

Katherine Lopez (Georgetown University)

Gerson Lúcio Vieira (Cancer Hospital Barretos)

Melissa Marzán-Rodríguez

Olayemi Matthew (Florida A\&M University)

Sapna Mehta (University of California San Diego)

Brittany C. Michel (Dana-Farber Harvard Cancer Center)

Maria Monberg (University of Texas MD Anderson Cancer Center)

Joseph Morales (University of California San Diego)

Olufunto Olusanya (Texas A\&M University)

Andrea Padilla (University of California San Diego Moores Cancer Center)

Luz Padilla (University of Alabama School of Public Health)

Janet Papadakos (Princess Margaret Cancer Centre)

Tina Papadakos (Princess Margaret Cancer Centre)

Ryan Park (University of California San Diego)

Sujata Patil (Memorial Sloan Kettering Cancer Center)

Robyn Pennella (St. Jude Children's Research Hospital)

Hao Pham (University of California San Diego)

Misty Pocwierz-Gaines (University of Nebraska Medical Center)

Naa Kwarley Quartey (Princess Margaret Cancer Centre)

Jocelyn Quiroz (University of California San Diego)

Sergey Radaev (National Cancer Institute)

Dhruti Ramchandani (City of Hope National Medical Center)

Owen Renault (Cancer Support Community)

Violetta Reznikov (Princess Margaret Cancer Centre)

Kern Rocke (The George Alleyne Chronic Disease Research Centre, A Unit of the

Caribbean Institute for Health Research)

Mariah Romero (University of California, San Diego)

Siddhartha Roy (Penn State College of Medicine)

Richa Ruwala (Cancer Support Community)

Saima Saleem (The Karachi Institute of Biotechnology and Genetic Engineering

(KIBGE), University of Karachi)

Diana Samoil (Princess Margaret Cancer Centre)

Gina Sanchez-Cruz (PSHU-MCC Partnership)

Ashna Shome (Rutgers New Jersey Medical School)

Ivan Silver (University of Toronto)

Z'Kera Sims (Moffitt Cancer Center)

Michael Skipworth (University of California San Diego)

Tyler Stringer (Princess Margaret Cancer Centre)

Arnethea Sutton (Virginia Commonwealth University School of Medicine)

Ewa Szumacher (Sunnybrook Health Sciences Centre)

Kay See Tan (Memorial Sloan Kettering Cancer Center)

Melissa Thomas (Ohio University Heritage College of Osteopathic Medicine)

Stephen Thomas (University of Maryland School of Public Health)

Aditi Tomar (Texas A\&M University)
P52

Plenary 4

Workshop 3

Workshop 1

$\mathrm{P}-14$

NCI Workshop

$\mathrm{P}-12$

$\mathrm{P} 28$

1C-5, 2A-5

Plenary 7

4B-3, P34

P1

P10

P19

$4 \mathrm{C}-3$

$1 \mathrm{~A}-3$

NCI Workshop, Plenary 3

P31

P53

P64

$2 \mathrm{C}-2$

$1 \mathrm{C}-4$

P35

P51

1B-2

P65

P39

4B-4

P6

1B-3, 3C-2

2A-1

P27

Workshop 2

Workshop 2, 2B-1, P32

3C-3

4A-2

P-13

P-11

P-59

2B-2, P26

$\mathrm{P} 20$

NCI Workshop

3B-4

$3 \mathrm{~A}-3$

2B-4

2A-3

P-18

P-7

3C-5

P2, P3

3B-1, 4C-2

P36

P45

Plenary 2

P40

P25

Workshop 2

P66

Workshop 1, 4C-1

4A-3

P47

Plenary 8

P57 
(continued)

Tracy Torchetti (Canadian Cancer Society)

Sally Trinh (University of California San Diego Moores Cancer Center)

1C-2, 2C-1, 3A-1, P5

Cindy Tsai (University of California San Diego Moores Cancer Center)

P61

Ben Umakanthan (Princess Margaret Cancer Centre)

P41

Rowan Ustoy (University of California San Diego Moores Cancer Center)

2B-3

P54

Pamela Valera (Rutgers State University of New Jersey)

$4 \mathrm{C}-4$

Nathan Vanderford (University of Kentucky)

Robin C. Vanderpool (NCI Health Communication and Informatics Research Branch)

1B-1

Milkie Vu (Emory University)

Monica Webb Hooper (National Institute on Minority Health and Health Disparities)

Lisa Tisdale Wigfall (Texas A\&M University)

Michelle Williams (George Mason University)

David Wiljer (University Health Network)

Yilin Xu (University of California San Diego Moore Cancer Center)

Allison Zambon (Fox Chase Cancer Center)

Xiao Zhang (Florida A\&M University College of Pharmacy-Economic, Social, and

Plenary 5

P15, P46

Plenary 1

P33

$2 \mathrm{C}-4$

Workshop 1

P58

$1 \mathrm{C}-3$

$\mathrm{P} 24$

Administrative Pharmacy (ESAP)

Ethan Yi Zhu (University of California, San Diego)

P17

Catherine Zivanov (Vanderbilt University School of Medicine)

$1 \mathrm{~A}-5$ 


\section{Workshops and Plenary Presentations}

\section{WORKSHOP 1}

Essential Skills in Cancer Education: Leadership, Leading and Influencing Change in Cancer Education - A Comprehensive Interactive Hands-On Workshop

Ewa Szumacher ${ }^{1}$, Krista Dawdy ${ }^{2}$, Maria Bishop ${ }^{3}$, Gilad Amiel ${ }^{4}$, David Wiljer $^{5}$, Kathleen Heneghan ${ }^{6}$, Jamal Khadar ${ }^{7}$

${ }^{1}$ Sunnybrook Health Sciences Centre, Toronto, ON, Canada, ${ }^{2}$ Odette Cancer Centre, Toronto, ON, Canada, ${ }^{3}$ Department of Medicine, the University of Arizona College of Medicine Program Director Section of Hematology/Oncology at the Southern Arizona Veterans Administration Health Care System (SAVAHCS) Chair of the Ethics Committee at the Southern Arizona Vetera, Tuscon, AZ, USA, ${ }^{4}$ Department of Urology, RAMBAM Healthcare Campus, Haifa, Israel, ${ }^{5}$ University Health Network, Toronto, ON, Canada, 'American College of Surgeons, Chicago, IL, USA, ${ }^{7}$ King Hussein Cancer Centre, Aman, Jordan

Abstract: Sound leadership skills in cancer education are required to ensure health care educators the necessary skill sets to shape the future of cancer education globally. This intensive 3-hour workshop will aid in the development of core leadership skills for interprofessional health care educators and trainees. Through in-course and post-course mentoring, participants will have an opportunity to develop leadership projects, implement them in their own educational practices and submit them for publication in the JCE. The overall goals of the course are to translate the principles of leadership into action; utilize change, networking and consensus building to set, align and achieve goals in an interprofessional setting; and engage the participants in leadership in cancer education and inspire initiative for change. The workshop design provides 1.5 hours for foundational learning on leadership in medicine drawing upon an international perspective. The course commences with an introduction, overview of objectives, presentation on the importance of leadership in medicine, and review of leadership styles, followed by 10 minute presentations facilitated by international leaders in medical education. A short breakout session will provide participants time to engage with each other, share knowledge and develop content learned through interactive participation of a provided case studies. The remaining time of the workshop allows for interactive work with faculty mentors on individual leadership projects the participants plan to introduce and implement in their own institutions. Participants will be encouraged to bring their own projects to work on to and will be mentored by the workshop leaders. Allied Health Professionals in Oncology and Trainees, medical surgical and radiation oncologists and are invited to attend.Objectives: At the end of the course the participants will be better able to: describe different leadership styles and how they are used in different situations; define the steps needed to initiate change in practice; address the challenges and communication gaps; develop productive/effective mentoring relationships and engage in scholarly projects to develop leadership skills necessary for oncology professionals. References: Bass, B. M., Avolio, B. J., Jung, D. I., \& Berson, Y. (2003). Predicting unit performance by assessing transformational and transactional leadership. Journal of Applied Psychology, 88(2), 207-218. Pype, P., Mertens, F., Helewaut, F., \& Krystallidou, D. (2018). Healthcare teams as complex adaptive systems: understanding team behaviour through team members' perception of interpersonal interaction. BMC Health Services Research, 18(1), 1-13. Sultan, N., Torti, J., Haddara, W., Inayat, A., Inayat, H., \& Lingard, L. (2019). Leadership development in postgraduate medical education: a systematic review of the literature. Academic Medicine, 94(3), 440-449.

\section{WORKSHOP 2}

\section{Difficult Conversations in Health Literate Care}

Tina Papadakos, Meredith Giuliani, Tylar Stringer, Janet Papadakos Princess Margaret Cancer Centre, Toronto, ON, Canada

Abstract: Health care providers (HCPs) often face difficult conversations. These conversations can have profound effects on both the HCP and the patient or caregiver. When delivered poorly, bad news can generate feelings of anger, fear, and mistrust in the recipient. Breaking bad news well is associated with positive patient health outcomes and can help reduce provider burnout and increase resilience.This workshop aims to provide theory and practice opportunities to support HCPs through difficult conversations. This course includes a pre-course online component (4 hours to complete over 1 month) and a 3 hour workshop during the ICEC conference. The online component includes 4 modules where participants have 1 month to complete videos, eLearning, reflections and group discussions in advance through the Princess Margaret Cancer Centre's online Learning hub Cancer Campus. The online modules include: (1) Health Literacy and Communication Strategies; (2) The Art and Science of Plain Language; (3) Seamlessness and Teams; (4) Resilience. The in-person workshop includes facilitated activities to reinforce online learning, an interactive exploration of communication styles and reflections, and role-play with video-recorded simulations with standardized patient actors. Objectives: The Difficult Conversations in Health Literate Care course aims to provide HCPs with health literacy and clear communication strategies to support patients and families including: Negotiating a mutual agenda with patients at the outset of encounters. Using communication related protocols and tools to support patients through difficult conversations. Eliciting questions from patients through a "patient-centered" approach. Avoiding using medical "jargon" in communication with patients. Taking a universal precautions approach to oral and written communication with patients. References: G. Hider and D. Hoepfer, Ethical Competency in Nursing and Allied Health, Kendall Hunt Pub Co, 2018. S. M. Alelwani and Y. A. Ahmed, "Medical training for communication of bad news: A literature review.," Journal of education and health promotion, vol. 3, p. 51, 2014.

\section{WORKSHOP 3}

Best practices in the development of web-enabled cancer patient education tools for improved decision making

Sarah Bass ${ }^{1}$, Linda Fleisher ${ }^{2}$, Cassidy Kenny ${ }^{2}$

${ }^{1}$ Temple University, Philadelphia, PA, USA, ${ }^{2}$ Fox Chase Cancer Center, Philadelphia, PA, USA

Abstract: Patient engagement is a "multi-dimensional psychosocial process resulting from a conjoint cognitive, emotional and behavioral enactment of individuals toward their health condition and management" including: what the patient does; what the patient thinks and knows; and what the patient feels. This workshop focuses on best practice approaches to the development of digital health tools for patient engagement, using case examples from the faculty's research (colorectal cancer screening, treatment decision making and clinical trial participation). Access to the Internet and mobile technologies are ubiquitous and e-health tools are widely available, yet few are related to cancer care and more specifically to clinical trials and most have not undergone rigorous development or evaluation. Designing salient digital health interventions (including web, mobile and device), is essential to high quality patient education and requires theoretically based formative research and user-center design with stakeholder input throughout impacting both content and technology design. This workshop will educate participants about the principles (theory-based, data and stakeholder-driven) and techniques (formative research methods and user-center design) to design digital health tools for 
patient activation. We will highlight these principles and techniques through 2-3 case examples, highlighting lessons learned. Questions (topics of interest, previous experience, challenges they are having in developing digital health interventions) will be asked at registration to guide the focus of the presentation and the development of case studies that participants will work on in small online groups applying these learnings. Outcomes include enhanced best practice knowledge and skills in digital health intervention development. Objectives: The participant shall be able to identify two lessons learned about digital health development. The participant shall be able to identify two barriers to patient accessibility to patient education. The participant shall be able to discuss how digital health can be used to promote informed decision making and patient activation. References: Kessel KA, Vogel MM, Kessel C, et al. Mobile Health in Oncology: A Patient Survey About App-Assisted Cancer Care. JMIR Mhealth Uhealth. 2017;5(6):e81. Published 2017 Jun 14. doi:10.2196/mhealth.7689. Kim H, Goldsmith J, Sengupta S, Mahmood, Powell P, Bhatt J, Chang C, Bhuyan S. (2017). Mobile Health Application and e-Health Literacy: Opportunities and Concerns for Cancer Patients and Caregivers. Journal of Cancer Education. 10.1007/s13187-017-1293-5.

\section{WORKSHOP 4}

\section{Advancing Antiracist Psychosocial Care to Address Financial} Hardship in Adolescent \& Young Adult Oncology

\section{Christabel Cheung}

University of Maryland School of Social Work and University of Maryland Greenebaum Comprehensive Cancer Center, Baltimore, MD, USA

Abstract: In recent decades, the oncology community has increasingly focused on adolescent and young adult (AYA) patients, diagnosed with cancer between ages 15-39 years, who are challenged to cope with lifethreatening illness, while navigating transitions from childhood to adulthood that are already characterized by tremendous growth, change, and stress. In particular, racial minority AYAs are known to simultaneously experience racism that leads to disparities. Antiracist, developmentallyappropriate approaches are needed to achieve equitable psychosocial care for AYAs. Furthermore, it is known that being young and a racial minority are both risk factors for financial hardship following a cancer diagnosis. Through presentation of didactic content, videos, case examples, and interactive exercises, participants will learn practical knowledge and skills towards advancing equitable psychosocial care for AYA cancer patients. Content will emphasize how to effectively employ an antiracist lens and conceptual framework for financial hardship, in order to implement psychosocial support that effectively combines knowledge and strategies from pediatric and adult settings, with uniquely AYA strategies that reflect their sociodemographic and contextual complexity. Objectives: At the end of the course the participants will be better able to: identify at least two biomedical challenges and two corresponding psychosocial/ contextual issues that are uniquely experienced among the AYA patient care population following a cancer diagnosis; and identify an antiracist approach (e.g., research practice or clinical skill) and a conceptualization of financial hardship that addresses the aforementioned biomedical and psychosocial issues/contextual social determinants of health for racial minority AYAs, who may be particularly vulnerable.

NCI TRAINING WORKSHOP: NCI'S SUPPORT FOR CANCER EDUCATION ACROSS THE ACADEMIC LIFESPAN: R25 AND UE5 PROGRAMS

NCI Workshop, Part 1: NCI supported R25 Cancer Research Education Grants Program
Sergey Radaev

National Cancer Institute, National Institutes of Health, Rockville, MD, USA

Abstract: This Workshop will provide information on the NCI Center for Cancer Training (CCT) R25 funding opportunities that support various types of cancer educational activities that complement and/or enhance the training of a workforce to meet the nation's biomedical, behavioral and clinical research needs. The NCI CCT has supported cancer education projects through the R25 funding mechanism for over 40 years. This Workshop will describe the current priorities of the NCI CCT R25 program and provide guidance to prospective applicants to help them prepare competitive grant submissions. The Workshop will provide an overview of the current NCI CCT R25 portfolio, which in Fiscal Year 2019 included 60 competing and non-competing awards with a total cost of \$14 million. The three current R25 funding opportunity announcements, namely, Courses for Skills Development (PAR-18-477), Research Experiences (PAR-18-478), and Curriculum or Methods Development (PAR-18-476) will be described in detail, including useful tips for prospective R25 applicants. Examples of ongoing successful R25 programs that have been competitively renewed will be showcased in separate R25 Workshop presentations by their Principal Investigators. Objectives: At the end of the Workshop the participants should be able to distinguish among the different types of the current NCI R25 funding opportunity announcements (FOAs) and select the most appropriate FOA for their cancer education projects as well as to apply the discussed tips and suggestions to prepare competitive R25 applications. References: Compaore, S., Ouedraogo, C.M., Koanda, S., Haynatzki, G., Chamberlain, R.M., Soliman, A.S. (2016). Barriers to Cervical Cancer Screening in Burkina Faso: Needs for Patient and Professional Education. Journal of Cancer Education, 31(4):760-766. PubMed PMID:26336956; PubMed Central PMCID: PMC4779069. Korczak, J.F., Chung, D.W., Rosemond, E., Von Hoff, D.D., Haspel, R.L., Waterbor, J.W., Chang, S., Ramirez, A.G., Perkins, S., Wiest, J., Lei, M. (2017). The National Cancer Institute R25 Cancer Education Grants Program: A Workshop Report. Journal of Cancer Education, 32(1): 3-10. PMID: 28064401; PMCID: PMC5290060.

NCI Workshop, Part 2: Enhancing Diversity through Early Intervention: the NCI R25 Youth Enjoy Science (YES) Research Education Program

Alison Lin

NIH/National Cancer Institute, Rockville, MD, USA

Abstract: As part of the NCI-supported R25 Cancer Research Education Grants Program workshop, I will present the diversity-focused R25 Youth Enjoy Science (YES) program. The presentation will describe the YES program's vision and goals, as well as provide information on current program status and application criteria. The YES program supports critical early intervention research educationthat engages underrepresented middle school, high school and undergraduate students and their teachers. The workshop allows attendees to become aware of NCI research education programs and learn about the various funding opportunities. The workshop will highlight different NCI R25s and describe the common themes as well as distinct goals of each program. The R25 YES program portion of this workshop will address questions about the unique YES program and highlight NCI support for diversity-focused training and education in cancer research. The YES portion of the workshop will include a presentation on the program goals, highlights and accomplishments, as well as identify important components of a successful application, review criteria and budget information. The attendees will be given an overview of how the YES program serves a critical role in generating a pool of well-prepared and enthusiastic young students who can move forward into the academic pipeline toward a cancer research career. The YES presentation will also address the urgent need to focus efforts and 
resources in enhancing workforce diversity, which necessarily starts with preparing young students from populations underreprented in biomedical research today. Objectives: The participant shall be able to understand the NCI Research Education Program opportunities, learn about the YES program requirements, become more aware of the importance of diversity-focused research education efforts, particularly in early intervention efforts, and collect a number of tips on formulating a successful application. The participant will also be provided with program contact information and be encouraged to contact the program officer for further conversation. References: Qua K, Papp KK, Junk DJ, Webb Hooper M, Berger NA. Youth Enjoy Science Program at the Case Comprehensive Cancer Center: Increasing Engagement and Opportunity for Underrepresented Minority Students. Ethn Dis. 2020;30(1):15-24. Published 2020 Jan 16. doi:10.18865/ed.30.1.15. Hudson L, Samons KM, Dicken HE, et al. A Brief Educational Intervention Enhances Basic Cancer Literacy Among Kentucky Middle and High School Students J Cancer Educ. 2020;10.1007/s13187-020-01696-3. doi:10.1007/s13187-020-01696-3

NCI Workshop, Part 3: NCI Awardee Skills Development Consortium (NASDC): Helping NCI-Funded Junior Faculty Succeed in Academic Cancer Research Careers

Jeannette Korczak

National Cancer Institute, Bethesda, MD, USA

Abstract: NASDC was funded by the NCI Center for Cancer Training (CCT) in 2020 as a pilot collaborative educational initiative to offer a suite of short courses to NCI-funded junior faculty investigators holding various types of "K", R00, R21, and first R01-equivalent awards. NASDC courses are focused on helping NCI grantees develop essential professional, research, and/or clinical skills that enhance their ability to establish and maintain successful independent academic cancer research careers. NCI-funded junior faculty investigators are at a critical career juncture where they are expected to successfully compete for additional grant support, as well as become academic leaders who teach, mentor, and manage a thriving research program in a hypercompetitive funding climate. This requires skills beyond those typically taught during graduate/medical or postdoctoral training. NASDC courses are intended to enhance such skills and help retain these investigators in cancer research through long, productive careers. The presentation will provide an overview of the cooperative agreement, consortium structure of NASDC, which is a unique collaborative educational endeavor undertaken by NCI CCT that is governed by a Steering Committee of NASDC PIs and NCI Project Scientist(s), who support and stimulate the awardees' activities by serving in a partnership capacity. Highlights of the four NASDC UE5 (cooperative agreement version of R25) course offerings and mentorship activities, as well as management of logistics and evaluation by the U24 Coordinating Center will be presented. Workshop attendees will become well-positioned to serve as NASDC ambassadors by promoting the UE5 courses to their NCI-funded junior faculty colleagues who can benefit from participating in them. By working together to assure the success of NASDC, it is hoped that this pilot initiative will be reissued so that Workshop attendees are able to propose additional needed programs and compete for future NASDC awards. Objectives: The Workshop participant shall be able to identify the NASDC goal of helping NCI-funded junior faculty succeed in their academic careers. The Workshop participant shall be able to identify the topics of the courses supported through the NASDC initiative. The Workshop participant shall be able to identify the eligibiity criteria to take NASDC courses, which depend on the type of NCI funding and current academic position of the course participant. References: Loyal J, Porto A, Camenga D. Creating a Program for Junior Faculty Professional Development: A Tool Kit. 2018 Med Ed Portal 14:10703. Pate A, Smith J, Caldwell D, Horace A, Zagar M. Development, Implementation, and Impact of a Collaborative Junior Faculty Engagement and Professional Growth Program: The Young
Faculty Leadership Initiative. 2018. Curr Pharm Teach Learn 10:352359.

\section{PLENARY PRESENTATION (Plenary 1 - Keynote Presenter)}

Plenary Presentation (Opening): Cancer Education in the Age of COVID-19: Disparities, Pandemics, and Infodemics

Monica Webb Hooper

National Institute on Minority Health and Health Disparities, Bethesda, MD, USA

Abstract: Community-engaged approaches to address unmet cancerrelated needs among underserved populations have never been more important. The unfortunate collision of two U.S. pandemics (namely COVID-19 and systemic racism) and a permeating infodemic, has exacerbated racial/ethnic disparities in preventable illness, heightened community distrust, and has the potential to reduce individual prioritization on reducing cancer risk. Authentic community-engaged approaches that seek to understand and address the social determinants of health are especially vital in the age of COVID-19, and are needed to dispel myths, increase trust, and reduce barriers to care. First, this presentation will discuss a three-phase community-engaged research effort as an exemplar of methods to understand multilevel determinants of distrust, unmet cancer-related needs, and health disparities in diverse populations. This project, completed just before the pandemic arose, has implications for ensuring that populations disproportionately affected are not further left behind and receive the needed culturally and community competent care. Second, the presentation will highlight the NIH scientific response and large-scale community engagement initiatives to address COVID-19 health disparities. Finally, overall conclusions and recommendations for the path forward will be discussed. Objectives: The participant shall be able to:Summarize research methods to understand multilevel determinants of distrust, unmet cancer-related needs, and health disparities in diverse populations. List ways to ensure that populations disproportionately affected are not further left behind and receive the needed culturally and community competent care. Describe the NIH scientific response and large-scale community engagement initiatives to address COVID-19 health disparities References: Guerrero N., De Leon C.F.M., Evans D.A., Jacobs E.A. Determinants of Trust in Health Care in an Older Population. J. Am. Geriatr. Soc. 2015;63:553\&ndash;557. doi: 10.1111/ jgs.13316. - DOI - PMC - PubMed. Singu S, Acharya A, Challagundla K, Byrareddy SN. Impact of Social Determinants of Health on the Emerging COVID-19 Pandemic in the United States. Front Public Health. 2020 Jul 21;8:406. doi: 10.3389/fpubh.2020.00406. PMID: 32793544; PMCID: PMC7385373.

PLENARY PRESENTATION (Plenary 2 - Samuel C. Harvey Lecture)

Learning Conversations - a window to a world of enhanced Cancer Education

Ivan Silver

Centre for Addiction \& Mental Health, University of Toronto, Toronto, ON, Canada

Abstract: Learning conversations are deep mutually inclusive and cocreated discussions between learners and teachers that enhance the context and understanding of experiential learning. This concept is derived from K-12 education and has influenced health professional education primarily with debriefing critical incidents, simulation learning and feedback. The concept has the potential for doing much more. Using a case study embedded in the care of a cancer patient where the social determinants of health are critical features, Dr. Silver will illustrate how learning conversations can deepen learning. Using contemporary learning theories 
and imagining the future of medical education in a Covid-19 world, he will recommend some practical pearls for cancer educators. Objectives: At the end of this presentation, participants will be able to: Describe learning conversations and their current impact on learning and teaching in the health professions. Discuss how learning conversations can deepen learning with complex topics and within organizations. Apply the learning conversation concept to your daily teaching, and use of feedback and debriefing. References: Learning Conversations: An Analysis of Their Theoretical Roots and Their Manifestations of Feedback and Debriefing in Medical Education. Tavares W, Eppich W, Cheng A, Miller S, Teunissen PW, Watling CJ, Sargeant. J.Acad Med. 2019 Jul 30. Online ahead of print. Reframing Professional Challenges Through Action Learning Conversations in Medical Organizations. Maura Striano. In European Perspectives on Transformation Theory pp 241-254. 2019.

\section{PLENARY PRESENTATION (Plenary 3)}

Diversity in cancer training - transforming challenges into opportunities

Alison Lin

NIH/National Cancer Institute, Rockville, MD, USA

Abstract: Building a diverse cancer research community and biomedical workforce depends on a strong foundation in training and education. Diversity in cancer training helps ensure the inclusion of new viewpoints, enhances innovation and creativity, and can assist in advancing cancer health equity. Over the past two decades, many diversity-focused training and career development programs (both federally and privately funded) have been instituted in response to the urgent need to enhance workforce diversity. These programs have been impactful in advancing diversity in cancer training, while also identifying areas for improvement. The National Cancer Institute's Center to Reduce Cancer Health Disparities developed and implemented the Continuing Umbrella of Research Experiences (CURE) program. N/A CURE, which supports underrepresented students and scientists, employs a holistic approach that promotes mentoring, professional support, and career skills building, all surrounding the centerpiece of individually mentored research experience. To date, CURE has trained over 4,000 students and scientists from middle school to the early investigator level. Objectives: Using CURE as an example, this presentation will explore the current landscape of diversity in cancer training and envision how to best achieve a cancer research training pool that mirrors the diversity of our communities. The participants will be able to identify common challenges in enhancing diversity as well as potential approaches to meet the challenges. The participants will also learn about diversity-focused training opportunities supported by NCI. References: The Condition of Education - Population Characteristics and Economic Outcomes - Population Characteristics Educational Attainment of Young Adults - Indicator May (2019) [Internet]. [cited 2019 Dec 18]. Available from: https:// nces.ed.gov/programs/coe/indicator_caa.asp. Carethers JM. Facilitating minority medical education, research, and faculty. Dig Dis Sci 2016;61:1436-1439. Rock D, Grant H. Why Diverse Teams Are Smarter. Harvard Bus Rev Digit Artic. 2016;p2-4. 3p. Valantine HA, Lund PK, Gammie AE. From the NIH: A Systems Approach to Increasing the Diversity of the Biomedical Research Workforce. CBE Life Sci Educ [Internet]. American Society for Cell Biology; 2016 [cited 2020 Jan 27];15.

\section{PLENARY PRESENTATION (Plenary 4 - EACE Plenary)}

\section{Training and Learning in Cancer in a Time of COVID}

Charles Kelly

European Association for Cancer Education, Newcastle upon Tyne, Tyne \& Wear, United Kingdom
Abstract: Education, both campus-based and online has changed in form and process dramatically and extensively since the onset of the COVID emergency. This has affected undergraduate, postgraduate, vocational and patient education, presenting multiple challenges to educators, not all negative as in some cases the emergency has facilitated an already initiated move to e-learning with increases in both motivation and resources. This presentation looks at how teaching and learning has changed in these different teaching scenarios and how patient education and opportunities for shared decision-making have been changed and what the educational future may bring. Objectives: The participant shall be able to be aware of the changes in educational processes and outcomes brought on by the COVID emergency at differing cancer education levels. The participant shall be able to measure and compare their own educational position for cancer against the changes which have occurred internationally, and how these may be relevant to their own cancer education model; and how that may change over the short medium and longterm. References: Onesti CE, Rugo HS, Generali D et al. Oncological care organisation during Covid 19 outbreak. ESMO open 5(4), 202008. Hwang ES balch CM, Balch GC et al. Surgical oncologists in the Covid 19 pandemic: Guiding cancer patients effectively through turbulence and change. Annals of Surgical Oncology 27(8) 2600-2613 2020 Aug. Yan F, Rauscher E, Hollinger A. The role of head and neck cancer advocacy organisations during the Covid 19 pandemic. Head \& Neck 42 (7): 1526 - 1532, 2020 Jul. Frey Mk, Blank SV. Coronavirus concerns: What do women with gynaecological cancer need to know during the Covid 19 crisis? Gynaecologic Oncology 158 (1): 32 - 33, 2020 07. Franco P,Kotchbati L, Siano M et al. Suggestions for Radiation Oncologists during the Covid 19 pandemic. BioMed Research International. 2020: 4892382, 2020.

\section{PLENARY PRESENTATION (Plenary 5)}

Using Cancer Communication to Address Social Determinants of Health

Robin Vanderpool

National Cancer Institute, Rockville, MD, USA

Abstract: Cancer communication is a cross-cutting theme of the cancer care contiuum, from prevention through end-of-life. It can improve the public's understanding of cancer information, enhance patient-providercaregiver interactions, bolster team communications within and between health care systems, bridge the gap between clinical and public health, translate research into clinical and public health practice, and promote health equity. Objectives: The participant shall be able to article how health-related communication and information can be used to address social determinants of health. The participant shall be able to identify how cancer communication research can be leveraged across the cancer control continuum to impact quality of life, health equity, and health outcomes. References: Natalie C. Benda, Tiffany C. Veinot, Cynthia J. Sieck, and Jessica S. Ancker, 2020: Broadband Internet Access Is a Social Determinant of Health! American Journal of Public Health 110, 1123_1125, https://doi.org/10.2105/AJPH.2020.305784. CamachoRivera M, Gonzalez CJ, Morency JA, Blake KD, Calixte R. Heterogeneity in Trust of Cancer Information among Hispanic Adults in the United States: An Analysis of the Health Information National Trends Survey. Cancer Epidemiol Biomarkers Prev. 2020;29(7):13481356. doi:10.1158/1055-9965.EPI-19-1375

\section{PLENARY PRESENTATION (Plenary 6)}

Cancer Disparities and Treatment Equity: Removing Barriers to Cancer Care in our Community

Christopher Lathan

Dana-Farber Cancer Institute, Boston, MA, USA 
Abstract: The goal of this talk is to review general concepts of health disparities in cancer care, and to describe the impact of socioeconomic status, and race on outcomes. A discussion of impact of structural racism told through my personal path to medicine, will focus on the barriers to becoming a physician due to race and class. Lastly the talk will describe a community based intervention. Objectives: 1 . Understand socioeconomic status impacts outcomes in cancer care even when access even when there is access to care. 2. Have a representation of other racial ethnic groups in clinical trials and tissue samples is important for the diffusion of precision medicine in cancer treatment. 3. Structural racism and classism prevent many from entering the field of medicine.

\section{PLENARY PRESENTATION (Plenary 7)}

\section{The Colors of COVID-19: Confronting Health Disparities During a Global Pandemic}

\section{Stephen Thomas}

University of Maryland School of Public Health, College Park, MD, USA

\begin{abstract}
A discussion of the history and present day state of structural inequality in healthcare, the excess burden that COVID-19 has placed on communities of color in the U.S., and bioethics in healthcare and research. Objectives: At the completion of the presentation, participants will be able to: 1) Describe the burdens of race and history underlying structural inequality and social determinants of health associated with the excess burden of COVID-19 illness and premature death among people of color in Maryland and around the nation. 2) List examples unconscious bias and how it may impact delivery of medical care and public health services. 3) Describe the difference between institutional racism in health care and tailored solutions with policy implications. 4) Discuss the origins of bioethics and its roots in the history biomedical research abuse of human subjects recruited from communities of color. References: Centers for Disease Control and Prevention, "COVID-19 in Racial and Ethnic Minority Groups,” accessed June 2, 2020, https://www.cdc.gov/ coronavirus/2019-ncov/need-extra-precautions/racial-ethnicminorities.html. Gray, TF, Cudjoe, J, Murphy, J, et al. Disparities in Cancer Screening Practices among Minority and Underrepresented Populations. Seminars in Oncology Nursing 33:2, May 2017, 184-198.
\end{abstract}

\section{Oral Abstract Presentations}

1A-1: Training Oncology Professionals in Health Equity: 20 Years of Palliative Care Education

Betty Ferrell ${ }^{1}$, Rose Virani ${ }^{1}$, Polly Mazanec ${ }^{2}$, Haley Buller ${ }^{1}$

${ }^{1}$ City of Hope, Duarte, CA, USA, ${ }^{2}$ City of Hope Medical Center, Mount Pleasant, SC, USA

Abstract: Culturally respectful care and attention to issues of health equity are important principles of all oncology care. These areas are of particular importance in advanced disease and end of life care when patients are most in need of physical and psychosocial support. This abstract describes the experience of the End of Life Nursing Education Consortium (ELNEC) project in training oncology professionals in areas of diversity and health equity. Since 2001, the ELNEC project was created to provide palliative care training to oncology professionals internationally through various curricula, including Core, Geriatric, Pediatric, Critial Care, and Communication. Over the years, the ELNEC curriculum has been adapted to include one of eight modules devoted to Culture and cultural-related content integrated in all modules. Strategies for teaching have included didactic content, role play, self-assessment, video and other forms of interactive and experiential learning. The ELNEC Project has addressed areas of diversity and health equity in over 250 courses in all 50 US states and 100 countries. Over 28,000 clinicians have been trained using the ELNEC curricula to become trainers to share this work with colleagues. The Cultural content has consistently been rated as $4.2-5$ (5=excellent) in all courses. Evaluation data from participants has included the need for more content on LGBTQ communities, diversity of religious beliefs, economic disparity, and the need for self-assessment. The ELNEC Project continues to provide palliative care training to oncology professionals and has expanded to include training materials focusing on conversations related to specific cultural topics. In addition, the ELNEC curricula continues to provide online education to better serve healthcare professionals in rural or underserving communities. Objectives: The participant shall be able to identify at least two ways that the ELNEC curricula addresses areas of health equity and diversity. References: DeWilde, C., \& Burton, C.W. (2017). Cultural distress: an emerging paradigm. Journal of Transcultural Nursing, 28(4), 334-341. Holguin, E., Hughes, F., \& Shamian, J. (2017). Transnational nursing organizations paving the way for global health: The International Council of Nurses as exemplar. In: W. Rosa (Ed.), A new era in global health: nursing and the United Nations 2030 agenda for sustainable development (Chapter 10, pp. 205-226). New York, NY, Springer Publishing Company.

\section{A-2: Interprofessional Communication Curriculum: Description} and Outcomes of a Cancer Education Program

Betty Ferrell ${ }^{1}$, Haley Buller ${ }^{1}$, Rose Virani ${ }^{1}$, Judith Paice ${ }^{2}$

${ }^{1}$ City of Hope, Duarte, CA, USA, ${ }^{2}$ Northwestern University; Feinberg School of Medicine, Chicago, IL, USA

Abstract: Communication skills are particularly important in culturally diverse populations and those with low health literacy. However, few healthcare providers receive formal communication skills training. The purpose of this abstract is to describe a train-the-trainer course for interprofessional clinician teams to prepare them to provide communication skills training at their home institutions. This abstract will describe the project and provide a summary of course evaluation from the pilot course. Organized by the 8 domains of the National Consensus Project (NCP) Guidelines for Quality Palliative Care, the Interprofessional Communication Curriculum (ICC) course includes skill-building exercises and interactive discussions to assist participants in integrating communication training into their clinical settings. Using a goal-directed method of teaching, faculty assisted teams in developing 3 institutional goals for providing communication skills training to others. Evaluation included an immediate post-course evaluation and follow-up at 9 months to track training efforts. The first interprofessional communication training course took plan in early 2018 with 26 teams consisting of primarily nurses, social workers, chaplains, and physicians. The pre-course survey revealed a lack of knowledge, resources to teach communication, and funding as participants' greatest challenges to improving communication at their institutions. Post-course evaluations demonstrated high satisfaction with the course. On a scale of 1 to 5 (1=lowest), the course met participant's expectations (4.8). A 9-month follow-up course revealed participants trained 1,246 additional healthcare professionals. The teams' pre-course goals focused on 1) staff education, training and mentorship, and 2) institution-wide system changes involving communication. Five ICC courses will be held in the next five years, nationwide, with $\mathrm{NCI}$ grant funding. An online curriculum is in development to supplement the in-person training and provide participants with additional materials to train their colleagues. Objectives: The participant shall be able to identify at least two ways to integrate communication skills training at their institutions. References: Dudley N, Chapman S, Spetz J. Communitybased palliative care leader perspectives on staffing, recruitment, and training. J Hosp Palliat Nurs. 2018;20(2):146-152. Donesky, D., Anderson, W.G., Joseph, R.D., Sumser, B., \& Reid, T.T. (2020). TeamTalk: Interprofessional team development and communication skills training. Journal of Palliative Medicine, 23(1), 40-7. 
1A-3: Educational Programs for Health Care Professionals on Cancer Screening in SGM Population

Andrew Lim, Kristin Dickinson, Robin Lally

University of Nebraska Medical Center, Omaha, NE, USA

Abstract: Sexual and gender minority (SGM) individuals are disproportionately affected by certain cancers. Medical and nursing education rarely includes content on cancer screening and/or prevention in this population. This integrative review aims to synthesize literature on educational programs for health care professionals (HCP) related to cancer screening for SGM. An integrative review methodology was used to guide a systematic search of five databases: CINAHL, PubMed, Embase, PsycInfo, and ERIC. Articles were included in this review if they covered any development, conduct, or evaluation of educational programs targeted at health professionals and/or students regarding cancer screening for SGM. Articles were excluded if they were not primary research, pertained to an educational program but not targeted at HCPs, were not SGM focused, or were not cancer-focused. Articles were analyzed by comparing the educational programs and their outcomes to draw conclusions about the state of the science. Six studies met the inclusion criteria. Three articles were pre- and post-test design, another utilized participatory action research, and the remaining described the development of competency training programs. Programs varied in length from 15 minutes $(n=2), 50$ minutes $(n=1)$, to 2 hours $(n=2)$. Half of the programs were designed to be delivered in person, while the rest utilized asynchronous delivery. Of the programs administered $(n=4)$, the reported outcomes included increased awareness and knowledge of SGM issues including cancer, increased confidence in providing care to SGM patients including cancer screening, and improved attitudes and behaviors towards caring for SGM patients. The dearth of information severely limits the ability to recommend any one educational program. However, existing programs appeared to show promise improving HCP knowledge, attitudes, and cancer screening behaviors in the SGM population. Further studies are needed of programs to educate HCPs on SGM cancer screening. Objectives: The participant will be able to: Identify at least three cancers that disproportionately affect SGM population; Summarize the need for SGM cancer prevention education provision in medical and nursing curricula; Identify at least two benefits of currently existing educational provision on SGM cancer screening References: Cathcart-Rake, E., Breitkopf, C. R., Kaur, J., O'Connor, J., Ridgeway, J. L., \& Jatoi, A. (2019). Teaching health-care providers to query patients with cancer about sexual and gender minority (SGM) status and sexual health. American Journal of Hospice \& Palliative Medicine, 36(6), 533-537. https://doi.org/10.1177/1049909118820874 Oller, D. (2019). Cancer screening for transgender patients: An online case-based module. MedEdPORTAL, 15, 1-5. https://doi.org/10.15766/mep_23748265.10796 Pratt-Chapman, M. L., \& Ward, A. R. (2020). Provider recommendations are associated with cancer screening of transgender and gender-nonconforming people: A cross-sectional urban survey. Transgender Health. https://doi.org/10.1089/trgh.2019.0083

\section{A-4: Handle with Care?: Incorporating Graphic Medicine into Healthcare Professional Training}

Lauren Huff, Natasha Icliates, Meredith Giuliani, Janet Papadakos

Princess Margaret Cancer Centre, Toronto, ON, Canada

\begin{abstract}
A qualitative study was conducted to explore the patient experience of getting a diagnosis of metastatic breast cancer. The study findings were translated into an in-person "reader's theatre" style professional development program, which has received an "overwhelmingly positive" response from participants. We aim to extend the reach of the program beyond the teaching hospitals of Toronto, and into hospitals in underserved communities by digitizing the content in a way that preserves its powerful emotional impact. The nine research-based scripts of the
\end{abstract}

training program were translated into a series of digital comics that can be viewed set to music and voiceover. These graphic medicine installments build compassion and awareness among healthcare practitioners, each addressing a separate issue associated with the patient experience of advanced cancer. The digital program is available online to facilitate wide access to healthcare professionals and can also be incorporated into existing training programs. The original qualitative study evaluated the program through user-feedback surveys. Most $(95 \%)$ agreed that the presentation (or others like it) have a place in hospital rounds. Most also felt that the issues presented were relevant to $(95 \%)$, and useful for thinking about $(93 \%)$, their clinical practice. The feedback included many positive comments such as: "I feel strongly that you should have this drama on tape, so that more patients, relatives and doctors can learn from it." An evaluation is underway to compare the original program and the new innovative digital program. This program has the potential to help patients and healthcare professionals alike by highlighting the emotional and social challenges faced when dealing with advancing disease. By providing free online access, these effects can be amplified to serve a larger demographic of patients and healthcare professionals. Objectives: The participant shall learn about graphic medicine as a medium for health professions education. The participant shall be able consider how to implement graphic medicine into standard education programs. References: Anthony Farthing and Ernesto Priego, "Graphic Medicine' as a Mental Health Information Resource: Insights from Comics Producers' (2016) 6(1): 3 The Comics Grid: Journal of Comics Scholarship, DOI: http:// dx.doi.org/10.16995/cg.74 Nayar, P. K. (2015). Communicable Diseases: Graphic Medicine and the Extreme. Journal of Creative Communications, 10(2), 161-175. doi:10.1177/0973258615597385.

1A-5: Introduction of Cancer Disparities and Survivorship Care Through a Post-Clerkship, Integrated Science Course for Medical Students

Catherine Zivanov ${ }^{1}$, Kaustav Shah ${ }^{1}$, Xuanyi $\mathrm{Li}^{1}$, Lourdes Estrada ${ }^{2}$, William Cutrer ${ }^{3}$, Mary Hooks ${ }^{3}$, Vicki Keedy ${ }^{3}$, Kimberly Dahlman ${ }^{2}$

${ }^{I}$ Vanderbilt University School of Medicine, Nashville, TN, USA, ${ }^{2}$ Vanderbilt University, Nashville, TN, USA, ${ }^{3}$ Vanderbilt University Medical Center, Nashville, TN, USA

Abstract: Despite being a leading cause of death in the United States, cancer receives disproportionately low curricular emphasis in medical education. Many students report low comfort providing oncologic and survivorship care. Additionally, racial and socioeconomic disparities in cancer outcomes have long been reported, yet no standardized cancer health disparities curriculum exists in undergraduate medical education. Post-clerkship integrated electives can be used to introduce concepts related to cancer disparities, culture, and survivorship care. In 2015, a Clinical Cancer Medicine Integrated Science Course (ISC) was launched for post-clerkship medical students at Vanderbilt University. Through this course, students gain exposure to cancer epidemiology, disparities in cancer screening and outcomes, and survivorship care while also learning foundational sciences related to cancer pathophysiology, molecular diagnosis, and precision therapy. Learning modalities include expert seminars, team- and case-based learning, personalized clinical experiences, and student presentations addressing common cancer misconceptions among patients. Since 2015, 72 medical students have enrolled in the Clinical Cancer Medicine ISC. After each week and at the end of the course, students were asked to complete anonymous, electronic, course evaluation surveys. Among 64 students who completed surveys, 92\% $(n=59)$ were satisfied with the overall learning experience and the mix of learning activities, and 91\% ( $\mathrm{n}=58)$ anticipated applying knowledge acquired in their future training and practice. Regarding seminars and activities addressing cancer epidemiology, disparities, survivorship care, and cancer misconceptions, the vast majority of students (>95\%) felt that the information presented was useful and relevant to their professional career. The Clinical Cancer Medicine ISC offers a unique opportunity to 
educate future physicians about disparities in oncologic outcomes, cultural misconceptions, and psychosocial and financial burdens of cancer care. Most students felt as though they benefited from material around cancer disparities, misconceptions, and survivorship care. Objectives: The participant shall be able to: Identify two barriers to the development and implementation of oncology and health disparities curricula for undergraduate medical education.; List three strategies for teaching social determinants of health, disparities in oncology screening and outcomes, psychosocial impacts of cancer, and survivorship care in an undergraduate medical oncology course. References: Siegel, RL et al. CA Cancer J Clin. 2020;70(1). Neeley, BC et al. J Cancer Educ. 2019;34(1):56-58. Mattes, MD et al. J Cancer Educ. 2016;31(4):679-686. Oskvarek, J et al. J Cancer Educ. 2016;31(3):529-532. Franzoi, MA et al. J Racial Ethn Health Disparities. 2019;6(5):909-916. Nazemi, A et al. Urol Oncol. 2019;37(10):784-790. Amini, A et al. Am J Clin Oncol. 2018;41(5):476484.Rhodin, KE et al. J Surg Res. 2020;253:214-223.

\section{B-1: Using Culturally-Focused Storytelling to Empower Appalachian Kentucky Youth to Understand and Address Cancer Disparities in Their Communities}

\section{Nathan Vanderford, Lauren Collett, Lauren Hudson, Chris Prichard} University of Kentucky, Lexington, KY, USA

Abstract: Kentucky has the highest cancer incidence and mortality rates the US with the greatest cancer burden being in the Appalachian region of the state $(1,2)$. These disparities are driven by social determinants of health $(2,3)$. Herein, through storytelling, youth of the region explored their understanding of cancer. We engaged 25 youth participants of the University of Kentucky's Appalachian Career Training in Oncology (ACTION) Program in a cancer storytelling exercise. The participants, all residents of Appalachian Kentucky, published culturally-framed essays describing their experiences with cancer, why they think cancer rates are so high in Appalachian Kentucky, and what they think can be done to lower the rates (4). We used content analysis to identify common themes in the essays regarding the types of cancers youth have observed, their feelings toward the causes of cancer in Appalachian Kentucky, and their proposed solutions that could lower cancer rates. Appalachian Kentucky youth observe occurrences of such cancers as lung, colon, breast and skin, which are known to be elevated in the region. The youth believe that low cancer education levels, tobacco use, and inability to access health care and/or low engagement with health care have the greatest influences on cancer rates. They suggest increased cancer education, tobacco control, and better health care access/engagement as solutions to lower cancer rates in Appalachian Kentucky. These observations and suggestions align with key social determinants of health that drive the high cancer rates in the region. Using storytelling, we empowered youth in Appalachian Kentucky to understand cancer through their personal experiences and their cultural framework. We also prompted youth to envision culturally-tailored community-based intervention strategies that could aid in reducing the cancer burden. Future work will implement and evaluate youth-driven, culturally-tailored intervention strategies in the area. Objectives: Identify Kentucky as ranking first in the nation in overall cancer incidence and mortality rates with the greatest burden of the disease being in the Appalachian region of the state. Describe the key social determinants of health driving Kentucky's cancer disparities. Recognize storytelling as an important method for individuals to conceptualize and communicate the burden of cancer around them as well as to envision possible culturally-tailored interventions. References: Cancer Statistics Center: Kentucky (2019) American Cancer Society; Rodriguez SD, Vanderford NL, Huang B, Vanderpool RC (2018) A social-ecological review of cancer disparities in Kentucky. South Med J 111:213-219. Charlton M, Schlichting J, Chioreso C, Ward M, Vikas P (2015) Challenges of rural cancer care in the United States. Oncology (Williston Park) 29:633-640. Vanderford NL, Hudson L, Prichard C. (2020). The Cancer Crisis in Appalachia: Kentucky Students take ACTION. Lexington, KY: The University Press of Kentucky
1B-2: Focus Group Discussions Among African Americans on Colorectal Cancer Screening in the Southern US

Olayemi Matthew ${ }^{1}$, Alexandria Washington ${ }^{2}$, Askal Ali ${ }^{3}$, Rima Tawk ${ }^{1}$, Gebre-Egziabher Kiros ${ }^{1}$, Cynthia Harris ${ }^{1}$, Kristin Wallace ${ }^{4}$, Clement Gwede $^{5}$, John Luque ${ }^{1}$

${ }^{I}$ Florida A\&M University, Tallahassee, FL, USA, ${ }^{2}$ Florida A\&M University, Institute of Public Health, Tallahassee, FL, USA, ${ }^{3}$ Economic, Social, Administrative Pharmacy-Florida A\&M University, Tallahassee, FL, USA, ${ }^{7}$ Medical University of South Carolina, Charleston, SC, USA, ${ }^{5}$ Moffitt Cancer Center, Tampa, FL, USA

Abstract: African Americans experience colorectal cancer (CRC) related disparities compared to other racial groups in the US. African Americans underutilize screening, are frequently diagnosed with CRC at a later stage, and encounter the highest mortality rates compared to other racial groups. In Florida, whites reported higher adherence to screening with colonoscopy in the last 10 years (69\%) compared to African Americans $(53 \%)$ in 2018. This study reports focus group data about colorectal cancer screening. Formative research was used to design a community health advisor intervention to increase stool-based screening in African Americans. The study team recruited four focus groups of African Americans ages 45 to 64 years old in northern Florida and included 21 women and 8 men. The questions asked participants to comment on knowledge, beliefs and attitudes about CRC and CRC screening, identify CRC screening resources, and provide feedback on an educational brochure. The focus groups generated qualitative data about CRC screening resources, feedback on colorectal cancer screening education, and preferences for different text messages to encourage screening. Participants provided feedback on the brochure and suggestions on improving content. They liked the colors and the graphics on human anatomy and the colon. They wanted a short testimonial on the brochure and photos featuring famous Black people who were CRC survivors. Community members indicated a preference for pictures of well-known community members rather than stock photos. Out of 13 possible text messages participants ranked the message, "screening for colon cancer saves lives" the highest. Limited information about CRC signs/symptoms and cancer prevention was reported. Participants mostly relied on accurate health information coming from their healthcare providers. There were some negative attitudes to CRC screening because of lack of insurance, fear, and embarrassment; however, this was more pronounced among men than women. Objectives: The participant will be able to: Identify preferences for patient education materials on colorectal cancer screening among African Americans in northern Florida. Identify two barriers related to social determinants of health for completing colorectal cancer screening among African Americans. References: Brittain K, Christy SM, Rawl SM. African American patients' intent to screen for colorectal cancer: Do cultural factors, health literacy, knowledge, age and gender matter? J Health Care Poor Underserved. 2016;27(1):51-67. doi:10.1353/ hpu.2016.0022. Coughlin SS, Blumenthal DS, Seay SJ, Smith SA. Toward the Elimination of Colorectal Cancer Disparities Among African Americans. J Racial Ethn Health Disparities. 2016;3(4):555564. doi:10.1007/s40615-015-0174-z

1B-3: Application of the Integrated Behavior Model to non-theorybased studies to identify determinants influencing catch-up HPV vaccination outcomes

Olufunto Olusanya, Jonathan Thomas, Aditi Tomar, Kemi Alonge, Lisa Tisdale Wigfall

Texas A\&M University, College Station, TX, USA

Abstract: Human papillomavirus (HPV) is most prevalent among people ages 15-24 years. Although recommended for heterosexual (ages through 21) and gay/bisexual males (ages through 26), HPV vaccination rates remain substantially lower than HealthyPeople2020 80\% target. In men, 
persistent infections from oncogenic HPV subtypes $16 \& 18$ contribute to anal, penile, and oropharyngeal cancers preventable through administration of HPV vaccines. Five databases - Medline (Ovid), Embase (Ovid), CINAHL (Ebsco), ERIC (Ebsco), and PsycINFO (Ebsco), were searched from January 2009 to July 2019 for empirical, research studies focused on HPV vaccination among young adult males ages 18-26 years from colleges and universities in the United States. The 14 studies incorporated into this investigation were excluded from a previous systematic review, specifically, because these studies did not utilize a psychosocial or motivational theoretical framework. Our analysis applied a comprehensive, multi-theory model - Integrated Behavior Model (IBM) to deductivelyinductively map the predictors for HPV-vaccination outcome measures. HPV vaccination rates were relatively low ranging from $4.3 \%-45.0 \%$. Our 14 studies depict HPV-vaccination outcome measures to include vaccine acceptability, vaccine initiation/completion, vaccine uptake, vaccine readiness, vaccination intention, and support for HPV vaccination mandate. Vaccination-related outcomes were influenced by factors such as knowledge and awareness; perceived susceptibility/severity/risks and barriers; attitudes and beliefs; social/peer influences; sexual habits; time constraints; vaccine cost; healthcare access; concerns about vaccine safety/side effects; as well as psychological traits e.g. managing threat/ uncertainty. Some participants reported lack of knowledge about HPV infection and vaccine, as a result, did not use educational materials and/or HPV vaccination services available at college/university health-centers. Health-centers in colleges/universities are uniquely positioned to share information on HPV vaccinations during wellness checks and/or immunization-scheduled visits. Efforts to mitigate cost barriers could include no-cost/reduced-cost vaccinations through Adult Safety Net programs. Also, study identified factors facilitating catch-up HPV vaccination outcomes that could be useful for specifying performance objectives to systematically plan/implement future programs. Objectives: The participant will be able to identify factors associated with facilitating catch up HPV vaccinations among males ages 18-26 years in United States college/university settings. References: Shannon CL, Klausner JD. The growing epidemic of sexually transmitted infections in adolescents: A neglected population. Curr Opin Pediatr. 2018;30(1):137-143. McQuillan GM, Kruszon-Moran D, Markowitz LE, Unger ER, Paulose-Ram R. Prevalence of HPV in adults aged 18-69: United states, 2011-2014. US Department of Health and Human Services, Centers for Disease Control and ...; 2017.

\section{B-4: Social Determinants as Barriers to Mammography Screening at a Community Cancer Screening Clinic}

Tyra Hooper, Nathaly Gonzalez, Rhonda Hamilton, Mary Mills, Jiachen Lu, Lucile Adams-Campbell, Chiranjeev Dash

Georgetown University/Lombardi Comprehensive Cancer Center, Washington, DC, USA

Abstract: Mammography screening can lead to reduced breast cancer mortality; however, screening disparities remain, and are driven by multiple social determinants of health. Using retrospective data from a community-based cancer screening program in Washington, DC, we examined the number and type of social determinant barriers among women navigated to mammography screening. We abstracted sociodemographic and healthcare access data from 554 women navigated through the Capital Breast Care Center (CBCC) in 2019. CBCC aims to reduce screening disparities through community education and patient navigation, primarily designed to address barriers associated with language, insurance, transportation, and healthcare access among medicallyunderserved women. The following 5 social determinant barriers were defined a priori based on the literature as dichotomous variables: Hispanic ethnicity or non-White race; non-English speaking; uninsured or underinsured; not having a primary care physician; and not having access to public or private transportation. Frequencies of individual barriers and clustering of barriers are reported. In 2019, the average age of women navigated to mammography was 50.35 years with $46 \%$ below the age of 50. A majority (60\%) were of Hispanic ethnicity and $29 \%$ were Black. Frequencies of social determinant barriers are as follows: Non-white/ Hispanic (98\%), Non-English speaking (65\%), uninsured/underinsured $(82 \%)$, no primary care access $(27 \%)$, no access to transportation $(4 \%)$. The frequencies of women in the study sample with $1,2,3,4$, and 5 barriers were $12 \%, 17 \%, 53 \%, 18 \%, 0.3 \%$, respectively. $71 \%$ reported $\geq 3$ barriers with younger women reporting more barriers than those above 50 (p Clustering of social determinant barriers was evident among those screened at an urban safety net clinic with younger women facing more barriers than older women. Our findings validate the importance of community-based outreach/education and patient navigation as important strategies to improve screening rates in health disparity populations facing multiple barriers. Objectives: The patient shall be able to understand the social determinants that can be barriers to breast cancer screening among underserved urban women; and the role of community education and patient navigation in addressing those barriers. References: 1 .Smith RA, Andrews KS, Brooks D, et al. Cancer screening in the United States, 2019: A review of current American Cancer Society guidelines and current issues in cancer screening. CA Cancer J Clin. 2019;69(3):184-210. 2. Coughlin SS. Social determinants of breast cancer risk, stage, and survival. Breast Cancer Res Treat. 2019;177(3):537-548. 3. Thornton RL, Glover CM, Cené CW, Glik DC, Henderson JA, Williams DR. Evaluating Strategies For Reducing Health Disparities By Addressing The Social Determinants Of Health. Health Aff (Millwood). 2016;35(8):1416-1423.

\section{B-5: Using Geolocation Analysis to Identify Gaps in Gynecological Supportive Care Services and Enable Targeted Educational Efforts in Rural Settings}

Henna Bhatia ${ }^{1}$, Andrea Calkins ${ }^{2}$, Brian Hendricks ${ }^{2}$, Nicole Stout ${ }^{3}$ ${ }^{1}$ West Virginia University, Morgantown, WV, USA, ${ }^{2}$ West Virginia University School of Public Health, Morgantown, WV, USA, ${ }^{4}$ West Virginia University Cancer Institute, Morgantown, WV, USA

Abstract: Rurality limits access to health care, a deficit that is magnified when specialty services are needed. This study characterizes existing supportive care services for individuals with gynecological cancer across West Virginia and explores geographic gaps. Findings will inform targeted education for providers and stakeholders to enhance local supportive services. Public records were used to identify state-wide licensed health care professionals in specialty areas of care including fertility management, rehabilitation medicine, sexual counseling and for supportive cancer care services such as cervical cancer screening sites, colposcopy clinics, and other $\mathrm{OB} / \mathrm{GYN}$ services. Visualization and mapping software was used to overlay county-level gynecological cancer incidence rates (cervical, ovarian, and corpus uterine) with geolocated supportive service centers. Cluster analysis was conducted using Local Indicators of Spatial Association (LISA). Bivariate analysis will be used to assess disease outcomes based on access to various supportive services. All statistical analysis is reported using a $95 \%$ confidence interval. A visual overlay of gynecological cancer incidence, by county, and supportive care service locations was created in Tableau (Figure 1 e.g. cervical cancer and screening sites). Preliminary GIS analysis identifies disparities between highincidence counties and prevalence of services including screening, counseling, and rehabilitative services. Preliminary LISA analysis identifies counties of statistical significance in West Virginia ( $p$ Analysis of specific county-level density of supportive care services and providers across the state is in process to evaluate gaps and a bivariate analysis will examine their impact on disease outcomes. As cancer survivorship grows in rural regions it is imperative to enhance local supportive care services. Considering resource constraints, targeted analysis of rural care access and gaps can enable precise deployment of educational interventions that enhance local resources. Future research should identify optimal educational strategies and medium to enhance and extend local, cancer-specific 
resources. Objectives: The participant shall be able to: 1 . Recognize the unique barriers to health care access that exist in rural areas. 2. Describe the ongoing supportive care needs that are anticipated among individuals living with and beyond gynecological cancers and identify providers and stakeholders in the local community that could meet those needs. 3 . Apply the methodology used for geolocation analysis to assess supportive care service gaps in a geographical region of interest. 4. Consider the resources and implementation strategies needed to educate local health care providers and stakeholders regarding the needs of gynecological cancer survivors. References: 1 . Wheeler SB, Davis MM. "Taking the bull by the horns": four principles to align public health, primary care, and community efforts to improve rural cancer control. The Journal of Rural Health. 2017;33(4):345-349. 3. Moss JL, Liu B, Feuer EJ. Urban/rural differences in breast and cervical cancer incidence: the mediating roles of socioeconomic status and provider density. Women's Health Issues. 2017;27(6):683-691. 4. Hung, P., Deng, S., Zahnd, W. E., et al. (2020). Geographic disparities in residential proximity to colorectal and cervical cancer care providers. Cancer, 126(5), 1068-1076.

\section{C-1: Community-Driven Development of Cancer Education in Northwest Arctic, Alaska}

Katie Cueva, Melany Cueva, Jennifer Schmidt

University of Alaska Anchorage, Anchorage, AK, USA

Abstract: Cancer is the leading cause of death for Alaska Native people, who are disproportionately burdened, both by cancer mortality and incidence, as well as associated risk factors. Health promotion efforts have the potential to alleviate disparities and reduce cancer risk factors among Alaska Native people. In response to community concerns about cancer, a project team based at the Institute of Social and Economic Research at the University of Alaska Anchorage traveled to three Alaska Native communities in the Northwest Arctic. Using a community based participatory action research approach, the project team facilitated 13 sharing circles in the communities, with 122 total participants, including high school students, school teachers/staff, health professionals, and community members. Participants overwhelmingly shared that cancer education for youth was a priority. Cancer prevention was identified as a priority for education, including information about smoking (mentioned in 11 of the sharing circles) and healthy foods ( 8 sharing circles). Participants also asked for information on types of cancers (8) and cancer treatments (6). Participants shared that the best ways for youth to learn were stories (11) (of local people, role models, and cancer survivors); visuals (8); and Northwest Arctic data and statistics (8). Participants also said there were no existing resources on cancer education in their communities (4). A community advisory board guided the development of relevant, culturally based online cancer education for use in schools. This presentation will discuss the process of respectfully engaging with three Alaska Native communities on a public health priority. Objectives: The participant shall be able to list culturally relevant engagement strategies with community members in the Northwest Arctic. The participant shall be able to identify ways sharing circle participants described best practices in cancer education with youth. References: I. Blake, P. Holck, and E. M. Provost, "Alaska Native Mortality Update: 2009-2013," Alaska Native Epidemiology Center, Anchorage, Alaska, Jun. 2016. A. M. Carmack, T. L. Schade, I. Sallison, E. M. Provost, and J. J. Kelly, "Cancer in Alaska Native People: 1969-2013, The 45 Year Report," Alaska Native Tumor Registry, Alaska Native Epidemiology Center, Alaska Native Tribal Health Consortium, Jun. 2015. S. H. Nash and D. G. Redwood, "Potentially Preventable Cancers Among Alaska Native People," Cancer Health Disparities, vol. 2, Jan. 2018.

1C-2: Improving It's My Life!, an interactive online tool, to increase cancer prevention awareness

Tracy Torchetti, Elizabeth Holmes

Canadian Cancer Society, Toronto, ON, Canada
Abstract: Research shows that individuals can reduce their cancer risk, but awareness about what causes cancer remains low (1,2). In 2014, the Canadian Cancer Society (CCS) created It's My Life! an interactive, evidence-informed tool to educate Canadians about cancer prevention and empower them to make a lifestyle change. It's My Life! is continuously evaluated to ensure that it is meeting the goal of increasing cancer prevention awareness. It's My Life! is continuously evaluated to ensure that it meets the goal of increasing cancer prevention awareness using qualitative feedback from user surveys as well as feedback from internal CCS stakeholders, a digital agency and software development company, a marketing agency and user testing. User testing was designed to assess whether the material is generally appropriate and easily understood for a diverse array of users including those in underserved populations. The diversity in the user testing sample included English and French users, male and female users, rural and urban users, users from LGBTQ+ community, variety of educational background and ages, and representations from multiple provinces in Canada. The collated feedback on the tool identified four key areas for updates: update statistics, improve usability, improve inclusivity and enhance content. New Canadian cancer statistics were included as well as the number and percentage of cancer cases in Canada due to modifiable risk factors $(3,4)$. Updated coding, new interactive features and revised content improved usability, inclusivity and content. It's My Life! is a tool that increases awareness and encourages behaviour change. Continued time and investment to enhance the tool is worthwhile as a cancer prevention education initiative. A similar model using technology to empower individuals could be explored to increase awareness about other topics along the cancer continuum. Objectives: Participants will learn how CCS continuously evaluates and updates an online prevention tool to ensure it remains effective among increasingly diverse users. References: Colditz GA, Wolin KY, Gehlert S. (2012). Applying what we know to accelerate cancer prevention. Sci Transl Med 4(127):127rv4. Environics Research Group for Canadian Partnership Against Cancer. (2008). The cancer prevention - attitudes, awareness and behaviours survey. Brenner DR et al. (2020). Projected estimates of cancer in Canada in 2020, CMAJ. 192:E199-205. Poirier A et al. (2019). The current and future burden of cancer attributable to modifiable risk factors in Canada: Summary of results. Prev Med 122:140-147.

1C-3: Bringing a clinical tobacco treatment program to the community.

Allison Zambon, Evelyn Gonzalez, Donna Edmondson Fox Chase Cancer Center, Philadelphia, PA, USA

Abstract: The national smoking rate is $14 \% 1$, but in Philadelphia the smoking rate is $22-25 \%$, the second-highest among large cities 2 . The rate of new lung cancers in Pennsylvania is 64.4 , significantly higher than national rate of 59.63. The most vulnerable population segments have the highest rates of smoking (e.g., those with lower education and incomes) 4 . Social determinants such as poverty, health care access, and transportation, influence tobacco-related disparities5. Comprehensive smoking cessation services can reduce disparities among groups most affected by tobacco use. NCI Comprehensive Cancer Center, Fox Chase Cancer Center (FCCC) has a well-established tobacco treatment program (TTP) - for patients. A community health needs assessment revealed an opportunity to address the community's needs by expanding the TTP to the community. To reduce barriers to participation among medically underserved groups most affected, FCCC offered the comprehensive cessation services and social support to community members, at community sites, free of charge. Participants learn about nicotine addiction, nicotine replacement therapies, smoking triggers, and strategies to stay smoke free. Participants complete pre/post-tests to determine their readiness to quit and addiction level. Follow-up surveys were administered at 2 and 4weeks, and 3-months post program. In 3 years, the program reached 61 people at four community locations. Participant characteristics: $64 \% \mathrm{fe}-$ male, $36 \%$ male; $49 \%$ African American, $48 \%$ white; $46 \%$ had a 
household income of $\$ 21,000-\$ 50,999$. Participants smoked for an average of 36 years, indicating many were highly addicted smokers. Fourteen participants $(23 \%)$ reported they had stopped smoking by last follow-up and twelve reported decreasing smoking. While the number of participants is typically low, those that complete the program have had success. Improvement measures included changing program times and developing new outreach strategies. This program shows how a tobacco treatment program can be adapted to meet community needs and reduce smoking levels of at risk populations. Objectives: The participant shall be able to: Summarize how Fox Chase adapted a clinical tobacco cessation program for community settings. Explain how to leverage community health needs assessment data to support outreach programming. Describe the impact this program had on the smoking habits of participants. References: 1 . Current Cigarette Smoking Among Adults in the United States. Centers for Disease Control and Prevention. https://www.cdc.gov/tobacco/ data_statistics/fact_sheets/adult_data/cig_smoking/index.htm. 2. Eichel L \& Camp-Landis S. Philadelphia Ranks High for Smoking and Other Unhealthy Behaviors. The Pew Charitable Trusts. https:// www.pewtrusts.org/en/research-and-analysis/articles/2017/07/25/philadelphia-ranks-high-for-smoking-and-other-unhealthy-behaviors. 3 . Pennsylvania State Data. American Lung Association. https:// www.lung.org/research/state-of-lung-cancer/states/pennsylvania. 4. Garrett BE, Dube SR, Babb S, McAfee T. Addressing the Social Determinants of Health to Reduce Tobacco-Related Disparities. Nicotine Tob Res. 2015;17(8):892-897. 5. Centers for Disease Control and Prevention. Best Practices User Guide: Health Equity in Tobacco Prevention and Control. Atlanta: DHHS, CDC, NCCDPHP, Office on Smoking and Health, 2015.

\section{C-4: ÁRBOLES Familiares Trainee Action Projects: Bringing Awareness and Referrals for Hereditary Breast and Ovarian Cancer into the Latino Community}

Kristi Graves ${ }^{1}$, Katherine Lopez ${ }^{1}$, Sabrina Sawhey ${ }^{1}$, Laura Moreno ${ }^{2}$, Jennifer Garcia ${ }^{2}$, Jennifer Ulloa ${ }^{3}$, Lina Jandorf ${ }^{3}$, Susan T. Vadaparampil ${ }^{2}$ ${ }^{1}$ Georgetown University - Washington, DC, USA, ${ }^{2}$ Moffitt Cancer Center, Tampa, FL, USA, 'Icahn School of Medicine, New York, NY, USA,

\begin{abstract}
In the ÁRBOLES Familiares training program, we train people working with Latinx communities to identify and refer individuals at high risk of hereditary breast and ovarian cancer (HBOC) to genetics professionals. We sought to describe the content and format of trainees' "Action Projects" developed to bring information to their communities. ÁRBOLES Familiares is a training program for bilingual (English and Spanish) community health educators, community health workers (called promotores in Spanish) or patient navigators. We aim to increase trainees' knowledge, self-efficacy and skills related to identification and referral of high-risk Latinas to genetic services. Training includes a 2 day in person workshop followed by online training modules. We introduce the Action Projects during the in-person workshop and provide time for active discussion, identification of resources and review of examples. Action Projects are due upon completion of the online training modules. Trainees are permitted to collaborate with one another on projects. To date, 28 trainees have submitted Action Projects with $82.1 \%$ designed for the community and $14.3 \%$ for healthcare providers. Action Projects ranged from 1-21 pages in length; $42.9 \%$ were in both English and Spanish, 25\% in English and 32.1\% in Spanish. A total of $60 \%$ of projects included HBOC information, 50\% reviewed family history and most included referral information for genetic counseling (96.4\%) and genetic testing (75\%). Results complemented trainees' 12-month behavioral outcomes: $100 \%$ reported educating community members about HBOC, $47 \%$ identified a community member at risk of $\mathrm{HBOC}$ and $44 \%$ navigated 1 or more patients to genetic services. Action Projects submitted as part of the ÁRBOLES Familiares Training Program represent a tangible outcome from the training that demonstrates attendees' application of their
\end{abstract}

knowledge and skills in the Latinx community. We hope these Action Projects will provide a valuable resource for both trainees and community members in the future. Objectives: 1 . The participant shall be able to identify at least two content areas presented in materials developed by ARBOLES Familiares trainees to educate the Latinx community about hereditary breast and ovarian cancer. 2 . The participant shall be able to identify barriers to genetic services within the Latinx community and apply information from the ARBOLES Familiares training program to consider ways to overcome these barriers in their community. References: San Miguel-Majors SL, Whitaker DE, Davis BC, et al. (2020). Education on Cancer Risk Assessment and Genetic Counseling to Address Cancer Health Disparities Among Racial/Ethnic Groups and Rural Populations: Implementing Culturally Tailored Outreach Through Community Health Educators. Journal of Genetic Counseling, Apr;29(2):243-246. doi:10.1002/jgc4.1272.Epub 2020 Mar21. PMID:32198903

McGuinness JE, Trivedi MS, Silverman T et al. (2019). Uptake of Genetic Testing for Germline BRCA1/2 Pathogenic Variants in a Predominantly Hispanic Population. Cancer Genet . Jun;235-236:72-76. doi: 10.1016/j.cancergen.2019.04.063. Epub 2019 Apr 24.

1C-5: Development of multilevel interventions to increase colorectal cancer screening and follow-up: Accelerating Colorectal Cancer Screening and Follow-up through Implementation Science in Appalachia

Aaron Kruse-Diehr ${ }^{1}$, Jill Oliveri ${ }^{2}$, Robin Vanderpool $^{3}$, Mira Katz ${ }^{1}$, Bin Huang ${ }^{1}$, Greg Young, ${ }^{2}$, Michael Pennell ${ }^{2}$, Paul Reiter ${ }^{2}$, Darrell Gray ${ }^{2}$, Darla Fickle ${ }^{2}$, Mark Cromo ${ }^{1}$, Melinda Rogers ${ }^{1}$, David Gross ${ }^{4}$, Ashley Gibson $^{4}$, Sue Russell ${ }^{4}$, Electra Paskett ${ }^{2}$, Mark Dignan ${ }^{1}$

${ }^{1}$ University of Kentucky, Lexington, KY, USA, ${ }^{2}$ Ohio State University, Columbus, OH, USA, ${ }^{3}$ University of Kentucky College of Public Health, Lexington, KY, USA, ${ }^{4}$ St. Claire Regional Medical Center, Morehead, KY, USA

Abstract: Appalachian Kentucky and Ohio have lower colorectal cancer (CRC) screening rates than in non-Appalachian regions. Multi-level interventions (MLI) are effective at increasing CRC screening. Herein we describe the development of MLIs in two federally-qualified health centers (FQHCs) in Appalachian Kentucky and Ohio and present preliminary findings from pilot implementation. Guided by the Social Determinants of Health Model, the Model for the Analysis of Population Health and Health Disparities, and Proctor et al.'s implementation model, we conducted formative research in 12 Appalachian Kentucky and Ohio counties sharing similar CRC cancer disparities. We conducted key informant interviews, created community profiles, identified clinic/ community champions, and conducted clinic environmental scans and data inventories. Our qualitative findings revealed CRC screening barriers at patient-, provider-, clinic-, and community-levels, reinforcing our decision to implement MLIs. We developed a menu of evidence-based interventions (EBIs) at each level, encouraging clinics to choose locally relevant, feasible, and implementable EBIs. In May 2019, project activities began in two FQHCs (Lewis County, Kentucky and Guernsey County, Ohio). Project members conducted implementation meetings with clinic staff and providers to review CRC statistics, discuss formative research findings, review baseline screening rates, and present the menu of EBIs for each implementation level. The clinics selected the following EBIs: patient education and FIT reminders/follow-up (patient level); provider education and feedback/assessment (provider level); and developing a screening protocol and improving electronic health record (EHR) reporting to increase annual wellness visits (clinic level). Community events focused on providing interactive screening education at recognized community locations. ACCSIS Year 1 formative evaluation yielded lessons about staff/provider workload, variability among rural clinics, and strategies to maximize EHR functionality. Years 2-5 will evaluate our MLI approach with a group-randomized trial in all 12 counties. We will 
measure screening/follow-up using clinic EHRs and self-reported screening via random-digit-dialed telephone surveys. Objectives: The participant shall be able to: 1 . Describe the burden of colorectal cancer in the central Appalachian population. 2. List at least two barriers to colorectal cancer screening encountered by rural populations. 3. Describe the added value of multi-level interventions for community cancer prevention and control. References: Geller BM, Skelly JM, Dorwaldt AL, Howe KD, Dana GS, Flynn BS. Increasing patient/physician communications about colorectal cancer screening in rural primary care practices. Med Care. 2008;46(9 Suppl 1):S36-S43. doi:10.1097/MLR.0b013e31817c60ea Proctor EK, Landsverk J, Aarons G, Chambers D, Glisson C, Mittman B. Implementation research in mental health services: an emerging science with conceptual, methodological, and training challenges. Adm Policy Ment Health. 2009;36:24-34. Rosenwasser LA, McCallHosenfeld JS, Weisman CS, Hillemeier MM, Perry AN, Chuang CH. Barriers to colorectal cancer screening among women in rural central Pennsylvania: primary care physicians' perspective. Rural Remote Health. 2013;13(4):2504.

2A-1: A Literature Review of the Barriers to End-of-Life Care among Hispanics with Advanced Cancer: Negative Connotations and Cost Fears

Andrea Padilla, Veronica Cardenas, France Nguyen-Grozavu, Georgia Robins Sadler

UCSD Moores Cancer Center, La Jolla, CA, USA

Abstract: End-of-life care (EoLC) encompasses ministrations given to those coping with terminal stages of illness. Cultural and linguistic barriers to accessing EoLC have been well reported. This narrative review specifically examines whether economic barriers and the terms themselves, "end of life care" and "hospice," hinders Hispanics' use of EoLC. Peer-reviewed research articles accessible in full text and published from 2006 to May 2020 were eligible for inclusion in this review. PubMed, CINAHL, PsycINFO, and Google Scholar databases were searched using such terms as: advanced cancer, Hispanic/Latino, endof-life care, hospice, palliative care, and barriers. The reference lists of identified articles were reviewed to identify additional eligible articles. New terms identified in the review were added to the searches. Articles were scored by theme(s) addressed, the type of study being described, whether the studies used objective, subjective, or a combination of measures to arrive at conclusions, and findings' generalizability. Over 70 articles were reviewed. Very few focused on whether the potentially negative connotations of the terms themselves, EoLC and Hospice, impacted Hispanics' use of EoLC. Similarly, few articles explored Hispanics' knowledge of the costs associated with EoLC and the degree to which their perceptions would influence their willingness to use EoLC for themselves or a loved one. The real/perceived cost of EoLC is often overlooked as a potential barrier since EoLC is covered by health insurance policies (Medicare and Medicaid). However, Hispanics have the highest uninsured rates within the United States, creating a potential barrier to utilizing EoLC. Researchers' limited focus on these barriers to Hispanics' use of EoLC make it impossible to assess the degree to which those two factors impact Hispanic Americans' use of EoLC services. Research is warranted to understand whether those factors impact the use of EoLC services, and, if so, how. Objectives: The participant shall be able to identify at least one additional barrier to accessing end-of-life care by Hispanics with advanced cancer.

The participant shall be able to identify one benefit that could result from addressing the negative stigma attached to the terms "end of life care" and "hospice." References: Gardner, D. S., Doherty, M., Bates, G., Koplow, A., \& Johnson, S. (2018). Racial and Ethnic Disparities in Palliative Care: A Systematic Scoping Review. Families in Society, 99(4), 301-316. https:// doi.org/10.1177/1044389418809083. Rising, M. L., Hassouneh, D. S., Lutz, K. F., Lee, C. S., \& Berry, P. (2018). Integrative
Review of the Literature on Hispanics and Hospice. American Journal of Hospice and Palliative Medicine $\AA$, 35(3), 542-554. https://doi.org/10.1177/1049909117730555

2A-2: The Unique Challenges and Barriers to Cancer Treatment for individuals with Autism: A review of the Literature and a Proposal for Future Modifications

Paola Cancino ${ }^{1}$, Georgia Robins Sadler ${ }^{1}$, France Nguyen-Grozavu ${ }^{1}$, Trisha Katz ${ }^{2}$, Leslie Carver ${ }^{1}$

${ }^{1}$ UCSD Moores Cancer Center, La Jolla, CA, USA, ${ }^{2}$ ELC Lab, La Jolla, CA, USA

Abstract: Autism Spectrum Disorder (ASD) is a prevalent developmental disability characterized by impairments in social communication and restricted interests. ASD patients have difficulty with abstraction and tend to think concretely, which may impact their ability to understand cancer diagnosis and treatments. We propose modifications to improve cancer outcomes for ASD individuals. This narrative review searched for best communication practices for individuals coping with Autism and cancer. Literature in English or Spanish, published between 2000 and 2020 available in full-text were utilized. Articles were found using PubMed, Science Direct, PsycINFO, CINHAL, and Google Scholar, and by reviewing the reference lists of eligible articles. Key search words included ASD, Autism, neurological disorders, learning disabilities, cancer, healthcare, education, and disparities. NIH, Autisms Speaks, and the CDC websites were also reviewed. We were unable to find any articles relating to ASD and cancer. Fourteen articles on cancer in people with learning disabilities (LD) were identified. Because no studies were found that addressed ASD and cancer, we instead examined the experience of individuals with $\mathrm{LD}$ and cancer, which shares some features with ASD. The incidence of cancer in people with $\mathrm{LD}$ was comparable to the general population. There were discrepancies in rates for cancer screenings between people with and those without LD. Factors that are possibly linked to this discrepancy included the inability to understand the implications of their diagnosis or their prognosis, fear of procedure, healthcare provider discomfort and suboptimal access to healthcare. These concerns likely manifest in ASD as well. As in ASD, those with LD also experience difficulties in communication. Programs that enhance communication between healthcare worker and patient are necessary for discussing diagnosis, prognosis and treatment. Programs for oncologists need to be implemented in order to improve the approach providers take for treating cancer patients with ASD. Objectives: The participants shall be able to recognize prevalence rates of cancer in patients with ASD (and or learning disability) from the literature review. Identify and determine the possible factors that are due to discrepancies in cancer screenings between those with learning disabilities (ASD) and those without. Finally, recognize the need for future implantations of programs for oncologist (and other health care physicians) that will care for patients with both ASD and cancer. References: Osborn, D. P. J., Horsfall, L., Hassiotis, A., Petersen, I., Walters, K., \& Nazareth, I. (2012). Access to Cancer Screening in People with Learning Disabilities in the UK: Cohort Study in the Health Improvement Network, a Primary Care Research Database. PLoS ONE, 7(8). doi: 10.1371/journal.pone.0043841. Tuffrey-Wijne, I., Bernal, J., \& Hollins, S. (2010). Disclosure and understanding of cancer diagnosis and prognosis for people with intellectual disabilities: Findings from an ethnographic study. European Journal of Oncology Nursing, 14(3), 224-230. doi: 10.1016/j.ejon.2010.01.021

2A-3: Colorectal Cancer Risk Perception and Knowledge among Caribbean University Students: A Cross-Sectional Study

Kern Rocke ${ }^{1}$, Selby Nichols ${ }^{2}$

${ }^{I}$ The George Alleyne Chronic Disease Research Centre, A unit of the Caribbean Institute for Health Research, Bridgetown, Barbados, W.I., Bridgetown, Barbados; ${ }^{2}$ Department of Agricultural Economics and Extension, The University of the West Indies, St. Augustine Campus, Trinidad, W.I., St. Augustine, Trinidad and Tobago 
Abstract: Colorectal cancer (CRC) remains a serious public health global concern. Perception of disease risk has been shown to be associated with engagement and adoption of healthy behaviors. Thus this study seeks to examine the association between cancer risk perception and knowledge of $\mathrm{CRC}$ and its risk factors among Caribbean university students. This was a cross-section study among undergraduate university students attending a premiere Caribbean university $(\mathrm{n}=1056)$. All research protocols and data collection instruments received institutional ethical approval. Purposive sampling was used to mimic the gender and faculty student distribution of the university. Data was collected using a self-administered questionnaire that focused on sociodemographics, knowledge of CRC, and its risk factors, dietary behavior, and cancer risk perception. Differences in demographic, lifestyle, knowledge, and food frequency across cancer knowledge categories were determined using the Mann-Whitney $U$ test. Bivariable and multivariable logistic regression models were used to assess the association between cancer risk perception and knowledge controlling for known confounders. Overall, the prevalence of students who perceived themselves at risk for developing CRC was $82.6 \%$ (95\% CI: 80.2, 84.7). Additionally, the prevalence of low knowledge of CRC and its risk factors was $35.9 \%$ (95\% CI: 33.0, 38.8). Students who were knowledgeable of CRC had significantly higher intakes of a westernized dietary pattern, lower intake of fruits and vegetables, were frequent smokers and consumed high amounts of alcohol. No significant differences among risk factors were observed across risk perception categories. The logistic regression model showed controlling for sociodemographics and lifestyle risk factors that students perceiving themselves at risk the disease were those who were knowledgeable of CRC (OR 1.89; 95\% OR:1.36, 2.64; $\mathrm{p}<0.001)$. This study shows there is a need to understand the risk factors for cancer in particular colorectal cancer in the Caribbean at an early stage of adulthood. Studies have shown that improving cancer knowledge at younger age aids in the adoption of healthier preventative risk behaviors and improves the quality of life. Objectives: The participant shall be able to understand the link between cancer risk perception and knowledge. They should be able to identify the prevalence of perception of risk among Caribbean students and to examine the differences between key lifestyle risk factors across CRC knowledge levels. Furthermore, you will understand the association between CRC risk perception and knowledge controlling for key modifiable lifestyle risk factors. References: 1. Gebresillassie BM, Gebreyohannes EA, Belachew SA, Emiru YK. Evaluation of Knowledge, Perception, and Risk Awareness About Breast Cancer and Its Treatment Outcome Among University of Gondar Students, Northwest Ethiopia. Front Oncol. 2018;8:501. 2. Osazuwa-Peters N, Tutlam NT. Knowledge and risk perception of oral cavity and oropharyngeal cancer among non-medical university students. J Otolaryngol Head Neck Surg. 2016 Jan 28;45:5. 3. Robb KA, Miles A, Wardle J. Perceived risk of colorectal cancer: sources of risk judgments. Cancer epidemiology, biomarkers \& prevention: a publication of the American Association for Cancer Research, cosponsored by the American Society of Preventive Oncology. 2007;16(4):694-702.

\section{A-4: Acceptance of HPV Vaccination Myths Among Hispanics in a US/Mexico Border Region: Literature review of Potential Interventions}

Priscila Chagolla ${ }^{1}$, Jesse Nodora ${ }^{2}$, Maria Milla ${ }^{3}$, Samir Gupta ${ }^{2}$, Sandip Patel $^{2}$, Georgia Robins Sadler ${ }^{2}$, Maria Martinez ${ }^{2}$, Corinne McDanielsDavidson $^{3}$

${ }^{1}$ San Diego State University, San Diego, CA, USA, ${ }^{2}$ UCSD Moores Cancer Center, La Jolla, CA, USA, ${ }^{3}$ UCSD Institute for Public Health, San Diego, CA, USA

Abstract: Human Papilloma Virus (HPV) vaccine series completion is lower in Hispanics than non-Hispanic whites (NHW). We validated an HPV vaccine myth scale and assessed whether the nature of misperceptions differed between the two groups in a large border county.
Separately, a literature search identified interventions that might correct these misperceptions. Spanish and English health surveys were mailed to a random sample of 5,000 households in San Diego County, California. Surveys included 12 items to assess the acceptance of HPV vaccination myths. Factor analysis was used to create a multidimensional scale and three subscales (age-related concerns, vaccine safety/efficacy, and association with sexual behaviors). Independent samples t-tests were then conducted to assess subscale differences by ethnicity (NHW vs Hispanics). In preparation for an intervention, databases (MEDLINE, PsycINFO, ERIC, and CINAHL) were searched using key terms (HPV vaccine, Hispanic, Beliefs, and intervention) to identify interventions to address myths and increase HPV vaccination uptake. Among returned surveys, 419 had complete responses for the myths scale. Overall, Hispanics had higher vaccine myth acceptance than NHW (p The literature review results reinforced our findings. Interventions were identified that specifically addressed identified misperceptions. Intervention components typically included outreach, education, and HPV vaccine access. Specifically, mother-daughter interventions have the potential to improve sexual health communication, which is an opportunity to provide accurate and culturally and linguistically appropriate information about HPV vaccination. Community-based interventions were also shown to significantly improve uptake. Hispanics demonstrated greater acceptance of HPV vaccination myths compared to NHW. Our literature review yielded several effective and culturally and linguistically appropriate interventions that can be implemented in the San Diego County Hispanic community to reduce myth endorsement, improve vaccine uptake, and ultimately reduce the burden of HPV-related cancers. Objectives: The participant shall be able to identify knowledge barriers to HPV vaccination as well as Identify culturally appropriate interventions to reduce myth endorsement of HPV vaccine. References: Barnack-Tavlaris, J. L., Garcini, L., Sanchez, O., Hernandez, I., \& Navarro, A. M. (2013). Focus group discussions in community-based participatory research to inform the development of a human papillomavirus (HPV) educational intervention for Latinas in San Diego. Journal of Cancer Education, 28(4), 784-789. Molokwu, J., Dwivedi, A., Mallawaarachchi, I., Hernandez, A., \& Shokar, N. (2019). Tiempo de Vacunarte (time to get vaccinated): Outcomes of an intervention to improve HPV vaccination rates in a predominantly Hispanic community. Preventive Medicine: An International Journal Devoted to Practice and Theory, 121, 115-120.

2A-5: Primary care provider perceptions of multi-level barriers to colorectal cancer screening and follow-up in Appalachian Kentucky

Aaron Kruse-Diehr ${ }^{1}$, Angela Carman ${ }^{1}$, Mark Cromo ${ }^{1}$, Melinda Rogers ${ }^{1}$, David Gross $^{2}$, Sue Russell ${ }^{2}$, Ashley Gibson ${ }^{2}$, Mark Dignan ${ }^{1}$

${ }^{1}$ University of Kentucky, Lexington, KY, USA, ${ }^{2}$ St. Claire Regional Medical Center, Morehead, KY, USA

Abstract: Screening rates are lower, and mortality rates higher, for colorectal cancer (CRC) in Appalachian Kentucky. As a foundation for implementing a multi-level intervention (MLI) in four primary care clinics in eastern Kentucky to address CRC screening disparities, we conducted key informant interviews to identify patient-, provider-, and clinic-level barriers. Members of the research team conducted a series of telephone interviews with 7 key informants from 4 primary care clinics in eastern Kentucky using a researcher-designed semi-structured interview guide. Questions explored clinic CRC screening practices, including patient education; perceived patient-, provider-, and clinic-side barriers to CRC screening; and clinic capacity to participate in research. Interviews lasted between 30 and 45 minutes, were audio-recorded, and transcribed verbatim. Two researchers independently coded transcripts to identify overarching themes and subthemes using a combination of deductive and inductive approaches. Coding discrepancies were resolved through a series of investigator debriefings. Overarching themes that emerged from thematic data analysis focused on patient, provider, and clinic level barriers to CRC screening. Patient-level barriers included fatalism, 
embarrassment and stigma, and fear of cancer outcomes, including a belief that treatment for CRC is "worse" than the diagnosis itself. Others included insurance concerns, rural transportation barriers, lack of patient knowledge about CRC, and competing medical priorities. Provider- and clinic-level barriers included physician preference for colonoscopy as preferred screening modality, unreliable educational material availability, inconsistencies tracking patient follow-up for a positive CRC screen via electronic health record (EHR), and difficulties integrating screening recommendations into clinic workflow. Patients have limited awareness of benefits of early CRC detection, and providers do not consistently suggest every screening modality. Strategies to improve screening must be integrated into clinic workflows. These findings reinforce the need for an MLI to address CRC disparities and will inform our implementation efforts in these clinics. Objectives: 1. Describe recommendations for colorectal cancer screening. 2. List factors in Appalachia that are barriers to screening References: Bardach SH, Schoenberg NE, Fleming ST, Hatcher J. Relationship between colorectal cancer screening adherence and knowledge among vulnerable rural residents of Appalachian Kentucky. Cancer Nurs. 2012;35(4):288-294. doi:10.1097/NCC.0b013e31822e7859. Attarabeen OF, Sambamoorthi U, Larkin KT, Kelly KM. Colon Cancer Worry in Appalachia. J Community Health. 2018;43(1):79-88. doi:10.1007/s10900-017-0390-Z.

\section{B-1: Difficult Conversations in Health Literate Care}

Tina Papadakos, Tylar Stringer, Naa Kwarley Quartey, Meredith Giuliani, Janet Papadakos

Princess Margaret Cancer Centre, Toronto, ON, Canada

Abstract: Many factors influence the patient-healthcare provider (HCP) relationship including the need to deliver complex information in an understandable manner, and breaking bad news with compassion. An HCP's competency in these domains can have a significant impact on patient outcomes as well HCP outcomes including increased resilience and reduced burnout. A course was developed to equip HCPs to use the principles of health literacy in difficult conversations. This study evaluated the impact of the course. The course aims to provide HCPs with health literacy and communication strategies including how to negotiate a mutual agenda with patients, avoiding using medical terms in communication with patients, and using communication-related protocols to support patients through difficult conversations. The course format is blended, includes simulation with standardized patient actors and ran as a pilot with an intact multi-professional team in a high acuity hematology unit for the evaluation. Participants were asked to complete an online survey at 3 time points: before the course, immediately after the course, and 3months post. The survey included the validated patient selfefficacy communication questionnaire (SE-12) as well as questions to evaluate participants' perceived competence and confidence in handling difficult conversations, and the program's perceived effect on their clinical practice. Thirty-one HCPS completed the course. Fifteen participants completed surveys at all three time points. This course provides a unique opportunity for HCPs to practice communication skills with an emphasis on health literacy and difficult conversations in the context of cancer in realistic scenarios with feedback from facilitators, peers, and the patient actors themselves. Objectives: A unique communications skills training program focussed on health literacy practices and communication. The impact of the course on a multi-professional team in a high acuity unit References: Warnock, C., Buchanan, J. \& TOD, A.M. ( 2017) The difficulties experienced by nurses and healthcare staff involved in the process of breaking bad news. Journal of Advanced Nursing 73( 7), 1632- 1645. doi: 10.1111/ jan.13252. Hider, G., \& Hoepfer, D. (2018). Ethical Competency in Nursing and Allied Health. Kendall Hunt Pub Co.
2B-2: Health Literacy Assessment of Cancer-Related Whiteboard Animations for Patients

Naa Kwarley Quartey, William Boateng, Kalsoom Shahzad, Eleni Giannopoulos, Lauren Huff, Meredith Giuliani, Janet Papadakos Princess Margaret Cancer Centre, Toronto, ON, Canada

Abstract: Whiteboard animations, which are videos where images are drawn on screen with accompanying narration [1], may leave patients feeling overwhelmed and distracted with the amount of information on the screen. This assessment will determine if publicly available cancerrelated patient whiteboard animations follow health literacy best practices. Videos were gathered from two commonly used search engines: Google and YouTube. The first thirty videos were retrieved from each search engine [2]. Twelve searches were conducted according to our search strategy to retrieve videos that met our inclusion criteria (English language, patient audience, cancer related). Videos were evaluated using the Patient Education Materials Assessment Tools for Audiovisual Materials (PEMAT-A/V) to assess understandability and actionability [3]; reading grade level was assessed using three readability calculators. A score of $80 \%$ or greater for understandability and actionability is acceptable in PEMAT-A/V. Reading grade level of 4-6 is acceptable according to best practices. 720 videos were retrieved and filtered based on inclusion criteria. Sixteen videos were included for analysis and were assessed using the PEMAT-A/V. PEMAT-A/V scores for understandability range from $42 \%-83 \%$ while actionability ranged from $0-100 \%$; readability grade levels ranged from 6-14. Only one video met the acceptable score for understandability, three videos met the acceptable score for actionability and only one video was at the acceptable grade 6 reading level. The majority of cancer-related whiteboard animation videos analyzed were below the acceptable level for understandability, actionability and readability. Whiteboard videos may be a creative method for knowledge dissemination, however those evaluated did not meet minimum best practice standards. This demonstrates that publicly available whiteboard animations may not adhere to health literacy best practices and thus may not be accessible to the populations they are designed to serve. Objectives: The participant will gain a greater appreciation for the importance of assessing publicly available resources for patients and the general population to ensure that resources adhere to best practices in health literacy. References: [1] Turkay S, et al. (2016). The educational impact of whiteboard animations: An experiment using popular science lessons. 7th Conference Proceedings: Digital Inclusions: Transforming Education Through Technology. Pgs 283-291. [2] Richards, Luke. "Video and Search: YouTube, Google, the Alternatives and the Future." Search Engine Watch, 9 Aug. 2019 https:// searchenginewatch.com/2018/06/27/video-and-search-youtube-googlethe-alternatives-and-the-future/ [3] PEMAT Tool for Audiovisual Materials (PEMAT-A/V). Content last reviewed October 2013. Agency for Healthcare Research and Quality, Rockville, MD. https://www.ahrq. gov/professionals/prevention-chronic-care/improve/self-mgmt/pemat/ pemat-av.html.

2B-3: Evaluating the Feasibility and Utility of Machine Translation for Patient Education Materials Written in Plain Language to Increase Accessibility for Populations with Limited English Proficiency

Ben Umakanthan ${ }^{1}$, Janet Papadakos ${ }^{1}$, Maria Anna Calamia ${ }^{2}$, Sarah McBain ${ }^{3}$, Jenney Wang ${ }^{3}$, Meredith Giuliani ${ }^{1}$, Shawn Amadasun ${ }^{1}$, Tina Papadakos ${ }^{1}$

${ }^{1}$ Princess Margaret Cancer Centre, Toronto, ON, Canada, ${ }^{2}$ Toronto Western Hospital, Toronto, ON, Canada, ${ }^{3}$ Cancer Care ON, Toronto, ON, Canada

Abstract: Populations with limited English proficiency (LEP) face comprehension barriers with health information that utilizes complex medical 
jargon and unfamiliar terms. The healthcare system is resourceconstrained with limited funding for translation. Evidence suggests that machine translation (MT) tools might hold promise for accurate translations of materials written in plain language. A sample of patient education (PE) materials with reading grade levels that meet plain language best practices were selected. The materials were translated into French, Italian, Spanish, Simple Chinese \& Traditional Chinese by medical interpreters (MIs) and by machine translation. Blinded quality review of translated materials was conducted by a different set of MIs using a standardized rubric that covers domains of fluency, adequacy, meaning, and severity. Ten PE materials were evaluated by MIs and data analysis is underway. Five sets of means will be calculated to represent the scores in each domain across the five target languages. To analyze the relationship between grade level readability score of the existing materials and translation accuracy scores, a Pearson correlation coefficient will be calculated. A multivariate analysis of variance (MANOVA) will be calculated to investigate if there is a statistically significant difference between MI and MT translated materials on all domains. It's well documented MT's performance in translating complex medical information is poor. MTs may improve translating materials that are written in plain language. The successful utilization of MTs for plain language text could increase accessibility of translated PE materials for vulnerable populations and improve health disparities that arise from miscommunication. Objectives: 1 . The reader will be able to assess the feasibility and utility of Google Translate to make patient education materials more accessible when created in plain language principles. 2 . The reader will be able to identify the difference in the quality of patient education materials translated by Medical Translators versus Google Translate. References: Chen, X., et al. (2016). Evaluating the accuracy of Google translate for diabetes education material. JMIR diabetes, 1(1), e3. Dew, K. N., et al. (2018). Development of machine translation technology for assisting health communication: A systematic review. Journal of biomedical informatics, 85, 56-67. Khoong, E. C. et al. (2019). Assessing the use of Google Translate for Spanish and Chinese translations of emergency department discharge instructions. JAMA internal medicine, 179(4), 580-582. Wu, Y., et al. (2016). Google's neural machine translation system: Bridging the gap between human and machine translation. arXiv preprint arXiv:1609.08144.

\section{B-4: COVID-19 \& Cancer Communication: An Evaluation of the Literacy Demand of Consumer Health Resources}

Violetta Reznikov ${ }^{1}$, Eleni Giannopoulos ${ }^{1}$, Meredith Giuliani ${ }^{1}$, Christine (Tina) Papadakos ${ }^{3}$, Janet Papadakos ${ }^{1}$

${ }^{1}$ Princess Margaret Cancer Centre, Toronto, ON, Canada, ${ }^{2}$ University Health Network, Toronto, ON, Canada

\footnotetext{
Abstract: The internet is widely used for accessing COVID-19 information and while abundant, it is also complex, inconsistent, and contradictory. This "infodemic" has repeatedly revealed poor health literacy to be an underestimated public health problem. This study aims to evaluate commonly visited COVID-19 and cancer websites against health literacy best practices. "Coronavirus and cancer" and "COVID-19 and cancer" were entered in Google Canada and the first 10 results were recorded. Inclusion criteria were: English language, targeted to cancer patients. Organization type was classified by industry. Each website was assessed for readability, understandability, actionability and accuracy. Readability was assessed using two calculators (SMOG and FRE) with an acceptable grade level of $80 \%$. The World Health Organization (WHO) guidelines were used to evaluate accuracy of knowledge and action statements with a score of $100 \%$ considered acceptable. Nine websites met inclusion criteria. The majority of websites were hosted by charitable organizations $(n=4)$ followed by medical societies $(n=2)$. All websites had reading grade levels above grade $6(\mathrm{M}=11.6, \mathrm{SD}=1.7)$. All websites scores below $80 \%$ for understandability $(M=68 \%$, range: $33-100, S D=14)$ and actionability $(\mathrm{M}=75 \%$, range: $33-100, \mathrm{SD}=22)$. None of the
}

websites had acceptable accuracy of knowledge statement scores $(\mathrm{M}=$ $41 \%, \mathrm{SD}=31)$ and only two websites had acceptable action statement scores $(M=65 \%, S D=34)$. It is challenging to cope with cancer, but these are exceptional times where patients are coping with the added stress of COVID-19. Our findings indicate that much of the consumer health information online does not meet health literacy best practices and therefore may not be accessible to patients seeking information. Objectives: The participant will be able to explain how to evaluate literacy demands of consumer websites. The participant will be able to describe how to conduct a comparative analysis of accuracy and consistency of messaging. The participant will be able to examine the availability of online consumer health information about COVID-19 and cancer that meets health literacy best practices. References: Paakkari, L., \& Okan, O. (2020). COVID-19: health literacy is an underestimated problem. The Lancet Public Health. doi: 10.1016/s2468-2667(20)30086-4. Papadakos, J.K., Charow, R.C., Papadakos, C.J., Moody, L.J., \& Giuliani, M.E. (2019). Evaluating cancer patient-reported outcome measures: readability and implications for clinical use. Cancer. 125 (8): 1350-1356.

2B-5: Establishing a framework for incorporating regular assessments of Organizational Health Literacy in cancer centres across Ontario

Janet Papadakos ${ }^{1}$, Eleni Giannopoulos ${ }^{1}$, Priyanka Jain ${ }^{2}$, Sarah McBain ${ }^{2}$, Meredith Giuliani ${ }^{1}$, Tina Papadakos ${ }^{1}$, Colleen Fox ${ }^{2}$

${ }^{I}$ Princess Margaret Cancer Centre, Toronto, ON, Canada, ${ }^{2}$ Cancer Care ON, Toronto, ON, Canada

Abstract: The Institute of Medicine describes 10 attributes of health literate healthcare organizations (HLOs) that make it easier for people to navigate, understand and use information and services to take care of their health. HLO measurement tools have been developed but which ones are most useful in practice is unknown. The purpose of this evaluation was to establish a framework for implementing a Province-wide evaluation of cancer centres as HLOs across Ontario. To identify HLO tools, and to determine which tools to use in the provincial evaluation, the grey and published literature were reviewed and fourteen HLO measures were found. Candidate measures were evaluated based on the following criteria: (1) the breadth of attributes addressed; (2) target population; (3) the setting; (4) HLO-relevant domains addressed; (6) test-retest reliability and; (7) whether the measure adhered to plain language standards. The validated Communication Climate Assessment Tool (C-CAT) was selected and piloted at one cancer centre to assess feasibility for Provincewide implementation. Five hundred and twenty-eight patients were approached over eight weeks and two hundred and fifty-one agreed to complete the survey, with a $48 \%$ response rate. The C-CAT electronic survey was e-mailed to four hundred and sixty-one frontline staff. Ninety-five staff surveys were completed, with a $21 \%$ response rate.. An electronic survey was e-mailed to fifteen executive leadership members and only one survey was completed with a $7 \%$ response rate. Findings indicated that over a short recruitment period, an adequate number of patient responses were obtained, likely due to clear survey design, use of plain language principles and quick time to complete. A different approach will be used in the Province-wide evaluation of frontline staff and leadership groups. Objectives: 1 . The participant shall be able to describe the process used to identify and review measures used to assess organizational health literacy. 2 . The participant shall be able to develop a framework for evaluating organizational health literacy in their organization. References: 1. Brach C. The Journey to Become a Health Literate Organization: A Snapshot of Health System Improvement. Stud Health Technol Inform. 2017;240:203-37. 2. Halverson JL, Martinez-Donate AP, Palta M, Leal T, Lubner S, Walsh MC, et al. Health Literacy and Health-Related Quality of Life Among a Population-Based Sample of Cancer Patients. J Health Commun. 2015;20(11):1320-9. 
2C-1: Inuusinni Aqqusaaqtara: Using e-learning modules to engage Inuit patients and healthcare providers

Tracy Torchetti ${ }^{1}$, Savanah Ashton ${ }^{2}$, Sipporah Enuaraq ${ }^{2}$

${ }^{1}$ Canadian Cancer Society, Toronto, ON, Canada, ${ }^{2}$ Pauktuutit Inuit Women of Canada, Ottawa, Canada, Canada

\begin{abstract}
Pauktuutit Inuit Women of Canada and the Canadian Cancer Society are working in collaboration to develop meaningful ways to support better health, reduce cancer incidence and increase prevention and early detection in the Inuit population. They have developed culturally appropriate cancer awareness tools and a toolkit to support community health representatives, healthcare providers and Inuit cancer patients. Inuusinni Aqqusaaqtara - My Journey - is a collection of resources developed for Inuit patients,caregivers and healthcare providers. The goal is to increase knowledge about cancer, improve communication between Inuit cancer patients and non-Inuit healthcare providers, enhance culturally appropriate care and improve overall quality of care for Inuit. Two new e-learning modules have been created to support these goals and to increase awareness of the resources. One e-learning module engages patients and caregivers through videos, what to expect during the cancer journey and other helpful information. The other engages healthcare providers around cultural sensitivity, how to include the patient resources into their practice and other key learnings. Both modules have been reviewed by target audiences and by experts and regional representatives through an advisory committee. The healthcare e-module will be accredited. An ongoing evaluation plan is in place for release. Cancer is a leading cause of death among Inuit populations. Compared to the general population of Canada, Inuit have a higher incidence of cancers. The resources, toolkits and e-modules will help to address the gap of culturally appropriate resources, low health literacy and lack of cultural competency among healthcare providers. Objectives: Participants will learn how Pauktuutit and CCS collaborated to produce e-modules to enhance and promote existing resources for patients and to educate and inform healthcare providers. They will also learn about the cultural and practical barriers Inuit face in seeking cancer care. References: Canadian Partnership Against Cancer. First Nations Cancer Control in Canada Baseline Report. Toronto, Ontario, Canada: Canadian Partnership Against Cancer; 2013. Health Council of Canada. Empathy, dignity and respect. Creating cultural safety for Aboriginal people in urban health care. Toronto, ON; 2012.
\end{abstract}

\section{C-2: Educating Massage Therapists to Engage in Skin Cancer} Primary Prevention Conversations with Clients

Lois Loescher, Kelly Heslin, Graciela Silva, Myra Muramoto

University of Arizona, Tucson, AZ, USA

\begin{abstract}
Skin cancer is an important focus of education efforts, affecting millions of Americans. Partnering with non-healthcare practitioners such as massage therapists (MTs) has potential for skin cancer risk reduction. Our objective was to evaluate 1) the feasibility educating MTs via online electronic training and 2) preliminary efficacy of the training. We adapted existing skin cancer education content for applicability to MTs and strategies from previous research on helping conversations as brief behavioral interventions. We assessed feasibility using data in Research Electronic Data Capture (REDCap), assessing preliminary efficacy of the training using established self-report surveys at baseline, immediately post-training, and 3 and 6 months post training. We collected and assessed data between 2018 and 2020. Ninety-five participants enrolled in the study; 73 (77\%) completed assessments at 6 months (overall attrition 23\%). Project satisfaction and e-training acceptability were high. Skin cancer risk reduction knowledge, personal behaviors (SSE, clinical skin examination; sun protection frequency), and practice attitudes (appropriateness and comfort with client-focused communication) all improved significantly and were sustained throughout the study. The e-training was feasible and
\end{abstract}

could be delivered online successfully to MTs. Participants were highly satisfied with, and accepting of, the e-training. The training has potential as an intervention in larger trials of MTs and skin cancer risk reduction. Objectives: The participant shall be able to describe the utility of partnering with the massage therapy community in skin cancer risk reduction efforts. The participant shall be able to describe the feasibility of recruiting massage therapists for an online training focused on skin cancer risk reduction activities with their clients. References: Trotter SC, LouieGao Q, Hession MT, Cummins D. Skin cancer education for massage therapists: a novel approach to the early detection of suspicious lesions. J Cancer Educ. 2014;29(2):266-269. Neufeld A, Anderson SK. Massage therapists and the detection of skin cancer in clients. Massage Today. 2013;February. LaPlante C. Early detection of skin cancer by massage therapists can save lives. American Massage Therapy Association Articles. 2008;March.

\section{C-3: Princess Margaret Global Oncology Leadership Development Program}

Meredith Giuliani ${ }^{1}$, Anna Dare ${ }^{2}$, Sandra Clarke ${ }^{1}$, Tylar Stringer ${ }^{1}$, Christine (Tina) Papadakos ${ }^{2}$, Danielle Rodin ${ }^{1}$

${ }^{1}$ Princess Margaret Cancer Centre, Toronto, ON, Canada, ${ }^{2}$ University Health Network, Toronto, ON, Canada

Abstract: Leadership skills are teachable yet rarely taught in oncology training programs. The Princess Margaret Global Oncology Leadership Development (GOLD) program was introduced to equip fellows with the skills, networks and opportunities to develop as leaders. This project evaluated participant satisfaction and change in perceived leadership confidence for the GOLD program. An interprofessional blended learning program was delivered over 18 weeks (10/2019-2/2020) including an introductory webinar, 2 online eLearning modules on negotiation and conflict management, and 4 days of live workshops. Thirteen speakers from 7 organizations delivered sessions focused on inter and intrapersonal, systems and organizational leadership skills. Immediate post-session participant satisfaction was measured through online surveys. Participants' change in confidence in 24 areas of leadership was measured through pre and post-program online surveys using a 7-point Likert scale. Average score across all areas and individual scores for the 5 largest differences were compared pre and post-program using paired t-tests. 31 participants from 21 countries participated. $100 \%$ of participants would recommend the program to a colleague and felt the program will help build global cancer leadership capacity. There was a significant increase in participants' mean change in confidence across the 24 areas of leadership from 4.1 pre to 5.2 post $(\Delta 1.1 ; \mathrm{p} \Delta 1.6 ; \mathrm{p} \Delta 1.6 ; \mathrm{p} \Delta 1.4 ; \mathrm{p}<$ $0.001]$. The GOLD program was positively received by participants and demonstrated early effectiveness in increasing participants' confidence across 24 different areas of inter and intrapersonal, systems and organizational leadership skills. This cohort will be followed to determine the long-term impacts of the program. Objectives: The participants should be able to articulate frameworks from conceptualizing leadership training and development. References: 1. Turner S, Seel M, Trotter T, Giuliani M, Benstead K, Eriksen JG, Poortmans P, Verfaillie C, Westerveld H, Cross S, Chan MK, Shaw T. Defining a Leader Role curriculum for radiation oncology: A global Delphi consensus study. Radiother Oncol. 2017 May;123(2):331-336. doi: 10.1016/j.radonc.2017.04.009. Epub 2017 Apr 25. 2. Lieff SJ, Yammarino FJ.How to Lead the Way Through Complexity, Constraint, and Uncertainty in Academic Health Science Centers. Acad Med. 2017 May;92(5):614-621.

2C-4: The HPV Vaccine Project ECHO Program: A pilot study of a telehealth program aimed at building clinician capacity to increase HPV vaccine coverage among adolescents

Michelle Williams ${ }^{1}$, Beth Dickson-Gavney ${ }^{2}$, Amy Ellis ${ }^{2}$, Taylor Christian $^{3}$, Shawn Rossi ${ }^{3}$, Letitia Thompson ${ }^{2}$ 
${ }^{1}$ George Mason University, Fairfax, VA, USA, ${ }^{2}$ American Cancer Society, Jackson, MS, USA, ${ }^{3}$ University of Mississippi Medical Center, Jackson, MS, USA

Abstract: The HPV vaccine is an effective tool for the primary prevention of HPV-related cancers. Geographic disparities in HPV vaccine coverage persist in states, such as Mississippi, that also have elevated HPV-related cancer incidence and mortality rates. The purpose of this pilot project was to assess barriers and facilitators to implementing a Project ECHO (Extensions for Community Healthcare Outcomes) program aimed at enhancing healthcare providers' capacity to increase HPV vaccine uptake among adolescent patients. We recruited healthcare providers in pediatrics clinics in Mississippi to participate in an HPV Vaccine Project ECHO program. The program consists of eight 1-hour sessions, during which experts lead didactic lessons focused on topics such as HPV vaccine coverage in Mississippi, strong provider recommendations, and using data from the Mississippi Immunization Information eXchange to track HPV vaccine coverage in their practices. Participants completed a pre-program survey and evaluations of each of the 8 sessions. The program began with 16 participants ( $n=3$ nurse practitioners; $n=1$ office manager; $n=3$ nurses, and $n=9$ physicians) enrolled. Participants completed a pre-program survey and an evaluation of each of the 8 sessions. Results of the session evaluations revealed that the program provided participants with the opportunity to develop skills that can be used to change their clinical practices. The teleconference format was a major facilitator of the program. Barriers to maintaining participant attendance over the course of the eight-month program included time constraints and redundancy of information. The HPV Vaccination Project ECHO program was effective at disseminating knowledge about the HPV vaccine, and enhancing providers' skills for delivering strong recommendations for the vaccine. The lessons learned from this pilot project will be used to revise the program curriculum and develop strategies for sustaining program participation. Objectives: The participant shall be able to identify facilitators to implementing a Project ECHO program. The participant shall be able to identify barriers to implementing a Project ECHO program. References: Brewer, N. T., Hall, M. E., Malo, T. L., Gilkey, M. B., Quinn, B., \& Lathren, C. (2017). Announcements Versus Conversations to Improve HPV Vaccination Coverage: A Randomized Trial. Pediatrics, 139(1). doi:10.1542/peds.20161764. Malo, T. L., Hall, M. E., Brewer, N. T., Lathren, C. R., \& Gilkey, M. B. (2018). Why is announcement training more effective than conversation training for introducing HPV vaccination? A theory-based investigation. Implementation Science : IS, 13(1), 57. doi:10.1186/s13012-018-0743-8

\section{C-5: Transition from In-Person workshops to Online Learning for Patient Navigation Training for Appalachia}

Mark Dignan ${ }^{1}$, Sharon Dwyer ${ }^{1}$, Elizabeth Rohan ${ }^{2}$, Dana White ${ }^{2}$, Georgina Castro $^{2}$, Reda Wilson ${ }^{2}$

${ }^{1}$ University of Kentucky, Lexington, KY, USA, ${ }^{2}$ Centers for Disease Control and Prevention, Atlanta, GA, USA

Abstract: The Appalachian region includes large areas with high rates of chronic disease, including cancer. Patient navigation $(\mathrm{PN})$ is a patientcentered intervention designed to address barriers to timely cancer screening and treatment. Results of a prior project indicated existing PN training programs did not adequately address issues specific to Appalachia. To address the gap in patient navigation training, we developed a culturallyrelevant patient navigation training program to be delivered in day-long workshops across the Appalachian region. We developed and delivered 20 in-person workshops to over 300 participants from 2015 - 2018, averaging 15 attendees per session. We evaluated the workshops using student evaluations, pre-post testing, and email follow-up. Although we provided multiple trainings in various locations throughout Appalachia, barriers to attendance remained. Process evaluation suggested that many barriers could be addressed through online learning. With support from an online learning vendor we developed an 8-module online learning program based on the in-person workshops. The modules require an average of 45 minutes to complete and include evaluation items to assess knowledge Modules include videos and exercises to maintain learner attention and provide Appalachian content. The content and evaluation data from implementation of the online learning program will be presented. Overall, the results suggest that participants valued access to the patient navigation training, the focus on Appalachian culture and that they found the content relevant and useful for their practice. An additional benefit was the ability to provide continuing education credits. Over 1500 hours of continuing education credit was provided Objectives: Participants at this presentation will be able to describe the Appalachian region and list factors associated with health disparities.Participants will be able to list guiding concepts for developing a patient navigation training for navigators working in Appalachia. Participants will be able to describe the benefits and challenges involved in providing training online. References: Freund KM, Battaglia TA, Calhoun E, et al. National Cancer Institute Patient Navigation Research Program: methods, protocol, and measures. Cancer. 2008;113(12):3391-3399. doi:10.1002/cncr.23960. Wells KJ, Battaglia TA, Dudley DJ, et al. Patient navigation: state of the art or is it science?. Cancer. 2008;113(8):1999-2010. doi:10.1002/cncr.23815

3A-1: CCS Cancer Information and Support Roadmap: identifying and addressing the information and support needs of underserved communities

Tracy Torchetti, Laura Burnett

Canadian Cancer Society, Toronto, ON, Canada

Abstract: The Canadian Cancer Society (CCS) is a national charity that offers support, information and practical programs for people affected by cancer. In 2019 CCS initiated the development of an evidence-based roadmap to identify the information and support needs of underserved or barriered populations including patients and caregivers in rural/remote communities, diagnosed with rare forms of cancer, diagnosed with advanced disease, LGBTQ2+, First Nations, Inuit and Metis, Adolescents and Young Adults, and non-English and non-French speaking individuals, and older adults. The roadmap used qualitive and quantitative data collection including literature review, environmental scan and consultation with key stakeholders. The Roadmap aims to provide a targeted, person-centred approach to the delivery of cancer information and support services to all Canadians. The roadmap methodology, process and outcomes that inform service adaptation will be presented. This includes the way engagement and co-design with patients and caregivers informed changes to CCS' current programs and services. Addressing barriers to better serve underserved communities requires dedicated resources, partnerships and time. CCS prioritized clients living with advanced disease, undertaking a review of its advanced cancer publication and support services to better meet their needs. But it's also implemented tactics that benefit multiple groups. Objectives: Participants will learn how CCS created its underserved roadmap, high-level outcomes of the research and how it plans to implement those tactics most likely to reduce barriers in accessing information and support services. References: Unmet care needs in people living with advanced cancer: a systematic review. Moghaddam N, Coxon H, Nabarro S, Hardy B, and Cox K. Supportive Care in Cancer, ISSN 0941-4355, 8/2016, Volume 24, Issue 8, pp. 3609 3622. Assessment of information needs and decision- making preferences in patients with metastatic cancer. Joseph D, Narasimhulu BC, Malik M, Ahmed SF and Valiyaveettil D. Annals of Oncology, ISSN 0923-7534, 11/2017, Volume 28, Issue suppl_10

3A-2: Making Educational Materials about Clinical Trials Accessible to Blacks and African Americans

Claire Saxton ${ }^{1}$, Kirstin Fearnley ${ }^{1}$, Maria Gonzalo ${ }^{1}$, Richa Ruwala ${ }^{1}$, Rachelle Theodore $^{2}$, Alyssa Jaisle ${ }^{1}$

${ }^{I}$ Cancer Support Community, Washington, DC, USA, ${ }^{2}$ CLT Strategic Solutions, Miami, FL, USA 
Abstract: Use of culturally sensitive approaches to enhance racial minorities' participation in clinical trials is important for eliminating health disparities. With the impact of low clinical trials participation among Blacks and African Americans in mind, the Cancer Support Community (CSC) culturally adapted its clinical trials educational materials to make information about cancer clinical trials more accessible to Blacks and African Americans affected by cancer. In 2019, CSC facilitated three in-person focus groups with African American cancer survivors $(\mathrm{N}=19)$ and their caregivers $(\mathrm{N}=10)$. The focus groups were used to determine if educational materials developed by CSC met the clinical trial information needs and were relatable to Blacks and African Americans affected by cancer. Participants gave feedback about the ease of understanding, applicability to Black and African American culture, and reactions to photos and images. Focus group transcripts were analyzed using inductive coding. Participant feedback was placed into categories aimed at answering pre-existing questions. Qualitative analysis of group recordings showed that: The booklets had useful information and helped participants understand clinical trials. There is an overall mistrust of the medical community, and unethical medical practices from the past should be addressed in the preface of the booklets. The images reflect Black and African American cultural themes (e.g. importance of family, religion, and food) but more images of people of color need to be included in professional roles including doctors and nurses. Findings revealed the booklets were perceived to be optimal tools to enhance knowledge and perceived importance about clinical trials. Additional barriers to clinical trial participation were also discussed in the groups. The booklets may lead to fruitful conversations about clinical trials among Black cancer patients, family members, and clinicians. Objectives: Identify culturally sensitive methods that can be applied to education materials about clinical trials. Understand the barriers to clinical trial participation among Blacks and African Americans. Identify two reasons why educational materials about clinical trials must address unethical clinical practices from the past. References: Hamel LM, et al. Barriers to Clinical Trial Enrollment in Racial and Ethnic Minority Patients With Cancer. Cancer Control. 2016 Oct;23(4):327-337. doi: 10.1177/107327481602300404.

Muñoz-Antonia T, et al. African Americans' and Hispanics' Information Needs About Cancer Care. J Cancer Educ. 2015 Jun;30(2):327-32. doi: 10.1007/s13187-014-0721-z. Nazha B, et al. Enrollment of Racial Minorities in Clinical Trials: Old Problem Assumes New Urgency in the Age of Immunotherapy. Am Soc Clin Oncol Educ Book. 2019;39:3-10. doi:10.1200/EDBK 100021. Wujcik D, et al. Recruitment of African Americans to National Oncology Clinical Trials through a Clinical Trial Shared Resource. J Health Care Poor Underserved. 2010 Feb;21(1 Suppl):38-50. doi:

\section{A-3: Information Needs about Cancer Clinical Trials among Black Patients and Caregivers}

Claire Saxton ${ }^{1}$, Kirstin Fearnley ${ }^{1}$, Maria Gonzalo ${ }^{1}$, Richa Ruwala ${ }^{1}$, Rachelle Theodore ${ }^{2}$, Alyssa Jaisle ${ }^{1}$, Owen Renault ${ }^{1}$

${ }^{1}$ Cancer Support Community, Washington, DC, USA, ${ }^{2}$ CLT Strategic Solutions, Miami, FL, USA

\begin{abstract}
Increased understanding about the most effective way to communicate with Black patients and caregivers, can raise awareness about cancer clinical trials, provide context about the importance of clinical research and how clinical trials impact outcomes both on a personal and societal levels. In 2019, Cancer Support Community (CSC) facilitated three regional in-person focus groups with predominantely female Black cancer survivors $(N=19)$ and their caregivers $(N=10)$ to gather insights about perceptions, knowledge, attitudes, and beliefs about clinical trials. Participants shared their information needs and opinions about clinical trials. Results were derived via qualitative analysis of group recordings. All discussions were transcribed and summarized under key themes. Findings assisted in the cultural adaptation of educational booklets about clinical trials to ensure that Black communities understand what
\end{abstract}

clinical trials are and how they can be accessed. Key findings suggest that educational materials about clinical trials should: Explain why is important for racial minorities to participate in clinical trials. Address barriers to participation such as: mistrust of the medical community, fear of side effects, disparities in access to healthcare, and lower socio-economic status. Provide information related to clinical trials that involved Black patients and the outcomes of those studies on the lives of participants. Provide information about cost of participation, financial support, and access to support services. These findings suggest the need to construct culturally tailored messaging around cancer clinical trials for Black patients and caregivers. Many participants were previously unaware of many of the topics discussed during the sessions but became more open to the idea of clinical trials after the discussion. Objectives: Understand the information needs about clinical trials among Blacks and African Americans. Identify perceptions, attitudes, and beliefs about clinical trials among Blacks and African Americans. Identify two barriers to participation in clinical trials among Blacks and African Americans. References: Hamel LM, et al. Barriers to Clinical Trial Enrollment in Racial and Ethnic Minority Patients With Cancer. Cancer Control. 2016 Oct;23(4):327-337. doi: 10.1177/107327481602300404. MuñozAntonia T, et al. African Americans' and Hispanics' Information Needs About Cancer Care. J Cancer Educ. 2015 Jun;30(2):327-32. doi: 10.1007/ s13187-014-0721-z. Nazha B, et al. Enrollment of Racial Minorities in Clinical Trials: Old Problem Assumes New Urgency in the Age of Immunotherapy. Am Soc Clin Oncol Educ Book. 2019;39:3-10. doi:10.1200/EDBK_100021. Wujcik D, et al. Recruitment of African Americans to National Oncology Clinical Trials through a Clinical Trial Shared Resource. J Health Care Poor Underserved. 2010 Feb;21(1 Suppl):38-50. doi: 10.1353/hpu.0.0251.

\section{A-4: Breast and Cervical Cancer Screening Outcomes in an Education and Patient Navigation Program in Rural and Border Texas}

Derek Falk ${ }^{1}$, Kristie Foley ${ }^{1}$, Kathryn Weaver ${ }^{1}$, Barbara Jones ${ }^{2}$, Catherine Cubbin $^{2}$

${ }^{1}$ Wake Forest School of Medicine, Winston Salem, NC, USA, ${ }^{2}$ The University of Texas at Austin, Austin, TX, USA

Abstract: This study examines breast and cervical cancer screening uptake for participants in a cancer education and patient navigation (PN) program for residents of rural and border counties in Texas by level of participation (education only, PN only, or education and PN). Data collected from March 1, 2012 to November 5, 2016 included 6,663 followup surveys. Logistic regression models assessed program participation on the odds of completing breast or cervical cancer screening. Analyses for breast cancer screening for women aged 40-74 and cervical cancer screening for women aged 21-64 were completed separately. Interaction effects between race/ethnicity/language and program participation were also tested. For women aged 40-74 $(\mathrm{N}=4,942), 58.4 \%$ reported a mammogram. Women who only received PN (OR: 6.06, CI: 4.87-7.53) or who participated in both the education plus PN program (OR: 3.33, CI: 2.77-4.02) had higher odds of mammogram screening compared to women who only received education. For women aged 21-64 (N=6,169); $37.7 \%$ received a Papanicolaou (Pap) test. Both education and PN (OR: 3.23, CI: 2.66-3.91) and PN only (OR: 2.35, CI: 1.88-2.93) groups had higher odds of Pap screening compared to those only receiving education. Significant interactions were observed between race/ethnicity/language and program and graphed for both screenings $(\mathrm{P}<0$.0001). $\mathrm{PN}$, alone or in combination with education, is an effective strategy to increase screening for breast and cervical cancer, beyond educational outreach efforts alone, among un-/underserved, racially/ethnically diverse women in rural and border Texas counties. PN serves as a resource to connect clinicians to rural and border populations. Objectives: The participant will be able to assess the variation in breast and cervical cancer screening uptake by level of program participation. References: Bernardo, B.M., 
et al., The efficacy and cost-effectiveness of patient navigation programs across the cancer continuum: A systematic review. Cancer, 2019. 125(16): p. 2747-2761. DuBenske, L.L., et al., Key elements of mammography shared decision-making: A scoping review of the literature. $\mathrm{J}$ Gen Intern Med, 2018. 33(10): p. 1805-1814.

3A-5: Mindfulness, Self-Compassion, and Quality of Sleep for Family Caregivers of Patients with Cancer: An Interventional Study

Laila Al-Daken

Sultan Qaboos University / College of Nursing, Al Khoudh, Muscat, Oman

Abstract: Family caregivers (FCs) spend more time providing care and assume more responsibilities for the patient, resulting in a higher level of physical, mental and emotional challenges. Purpose: to examine the effects of brief Mindfulness-Based Interventions Interventions (MBIs) and Educational Intervention (EI) on enhancing mindfulness, selfcompassion and quality of sleep for FCs' of patients with cancer. A quasi-experimental, pre-test-post-test study was used. Two interventions were conducted, the first intervention is brief MBIs and the second intervention group attended an EI The outcome variables were measured using: 1) the Arabic version of Mindful Attention Awareness Scale (MAAS); 2) the Arabic version of Self-Compassion Scale-Short Form (SCS-SF); 3) the Arabic version of Pittsburg Sleep Quality Index (PSQI). All participants completed the measures in the pre-test and post-test time. At the end of the interventions, the results of the paired samples t-test indicated that FCs in the mindfulness group demonstrated significant improvements in measures of mindfulness, self-compassion and quality of sleep with a medium to large effect size (Cohen d between 0.36 and $2.01, \mathrm{P}<.001)$. The independent samples t-test indicated that these improvements in the educational group were much less than improvements in the mindfulness group. No significant improvement in the quality of sleep was found at the end of EI. The findings provide preliminary support for effectiveness of MBIs and EIs as a supportive care for FCs of patients with cancer. Objectives: By the end of this presentation participants shall be able to: Identify the effect of mindfulness based interventions on enhancing mindfulness, self-compassion, and quality of sleep among FCs of patients with cancer. Identify the effect of educational interventions on enhancing mindfulness, self-compassion, and quality of sleep among FCs of patients with cancer References: Van den Hurk DG, Schellekens MP, Molema J, Speckens AE, van der Drift MA (2015). Mindfulness-based stress reduction for lung cancer patients and their partners: results of a mixed methods pilot study. Palliative Medicine:0269216315572720. Al Daken LI, Ahmad MM (2018). The implementation of mindfulness-based interventions and educational interventions to support family caregivers of patients with cancer: A systematic review. Perspectives in psychiatric care.

\section{B-1: The cost of a pamphlet: a provincial evaluation of cancer edu- cation resource production}

Janet Papadakos ${ }^{1}$, Diana Samoil ${ }^{1}$, Eleni Giannopoulos ${ }^{1}$, Sarah McBain ${ }^{2}$, Nicole Mittman ${ }^{3}$, Tina Papadakos ${ }^{1}$, Colleen Fox ${ }^{2}$, Lesley Moody ${ }^{2}$, Robin McLeod $^{2}$

${ }^{1}$ Princess Margaret Cancer Centre, Toronto, ON, Canada, ${ }^{2}$ Cancer Care Ontario, Toronto, ON, Canada, ${ }^{3}$ Sunnybrook Research Institute, Sunnybrook Health Sciences Centre, Toronto, ON, Canada

Abstract: Ontario Health (Cancer Care Ontario) is responsible for ensuring the quality and distribution of patient education resources in Ontario, Canada. A financial analysis was therefore conducted by the organization to evaluate the costs associated with developing and reviewing pamphlet patient education materials across provincial cancer centres. While patient education often produces a positive return on investment; this market remains highly unregulated, such that the personnel, time and capital allocated to the production of educational materials varies significantly across the health-care system. Patient education leads at fourteen cancer centres completed a survey measure, reporting estimates of the number of hours spent on pamphlet development and review activities at their respective centre and identifying the personnel responsible for the execution of each activity. Follow-up interviews were conducted to clarify the estimates and derive consensus regarding personnel involvement. The number of hours expended on each activity, reported on a per centre basis, was then combined with average salary data for the personnel involved to derive an estimate of the total costs (2019CAN\$) associated with pamphlet materials development and review. The number of hours spent developing and reviewing resources varies substantially between cancer centres. The greatest per pamphlet costs are attributed to writing content $(\overline{\mathrm{x}}=\$ 850.00)$ in the development stage and reviewing content with subject matter experts $(\overline{\mathrm{x}}=\$ 340.00)$ in the review stage. Each cancer centre spends on average $\$ 5,600.00$ developing $(\bar{x}=\$ 4,500.00)$ and reviewing $(\overline{\mathrm{x}}=\$ 1,100.00)$ one pamphlet resource. This cumulates to an average per annum spending of $\$ 85,200$ for pamphlet development $(\overline{\mathrm{x}}=\$ 65,400.00)$ and review $(\overline{\mathrm{x}}=\$ 19,800.00)$ at each centre across the province. Variations between centres in the costs and time allocated to patient education resource production can result in the duplication of efforts, an oversaturated resource market, and inconsistencies in patient access to quality educational materials as a function of their geographic location and closest cancer centre. Results will be used to produce recommendations to maximize scare fiscal and human resources in the health-care setting and to optimize patient education efforts across the province of Ontario, Canada. Sharing mechanisms will be employed across cancer centres to streamline the process of patient education development and review and to promote uniform resource quality. Objectives: 1 . The participant shall be able to identify at least three key personnel involved in the development and review of patient education resource production. 2. The participant shall be able to synthesize at least two suggestions regarding the optimization of the production of patient education materials in the cancer context. 3 . The participant shall be able to synthesize at least two implications that optimizing the production of patient education materials would have regarding equitable access to cancer education materials. References: 1 . Masters R, et al. Return on investment of public health interventions: a systematic review. J Epidemiol Community Health. 2017; 71(8):827-834. 2. Stenberg U, et al. Health economic evaluations of patient education interventions a scoping review of the literature. Patient Education and Counseling. 2018;101(6):1006-1035. 3B-2: Reinventing the Wheel: The Incidence and Cost Implication of
Duplication of Effort in Patient Education Materials Development

Janet Papadakos ${ }^{1}$, Eleni Giannopoulos ${ }^{1}$, Leta Forbes ${ }^{2}$, Diana Samoil ${ }^{1}$, Priyanka Jain ${ }^{2}$, Sarah McBain ${ }^{2}$, Nicole Mittman ${ }^{3}$, Colleen Fox ${ }^{2}$, Lesley Moody $^{2}$ Robin McLeod ${ }^{2}$

${ }^{1}$ Princess Margaret Cancer Centre, Toronto, ON, Canada, ${ }^{2}$ Ontario Health (Cancer Care Ontario), Toronto, ON, Canada, ${ }^{3}$ Sunnybrook Research Institute, Sunnybrook Health Sciences Centre, Toronto, ON, Canada

Abstract: Development of patient education (PE) materials can be a costly and resource-intensive endeavor, and there is currently no formal process or mechanism for sharing. This may lead to potential duplicated efforts if this is not realized. This study explored the incidence and economic impact of redundant PE efforts. PE leaders from cancer centres across Ontario were asked to submit their collections of systemic therapy PE materials to Ontario Health (Cancer Care Ontario). Materials were reviewed if they were in print format, were English and were related to systemic therapy. Materials were categorized by topic and were coded as Redundant (more than one other material exists on the topic), Adapted (material was adapted from an existing material) or Unique (no other material addresses the topic). Descriptive statistics were used to describe the proportion of each category. A separate analysis was conducted to 
determine the cost of redundant content. 304 materials were included in the analysis. Findings indicated that more than half of the materials were Redundant ( $\mathrm{n}=166,55 \%)$, a small proportion were Adapted $(\mathrm{n}=27,9 \%)$, and less than half were Unique $(\mathrm{n}=111,37 \%)$. The majority of PE materials are amenable to adaptation meaning that the content was not dependent on a specific context $(n=283,93 \%)$. The most common Redundant topic was safe handling of chemotherapy $(n=27)$. The Unique topics included management of brain fog, and treatment induced menopause. The opportunity for cost savings among cancer centers is estimated at approximately $\$ 700,000.00$ for systemic therapy materials alone. Our findings indicate that there is a need to standardize the process for developing patient education materials. Creating mechanisms of sharing can help facilitate equal access by different cancer centres to educational materials, and can result in significant resource and cost savings. Objectives: 1 . The participant shall be able to describe the economic impact of redundancy and duplicated efforts in systemic therapy patient education materials developed and utilized in Ontario cancer centres. 2. The participant shall be able to identify several redundant and common versus unique systemic therapy patient education topics. 3 . The participant shall be able to support the need for implementation of a widespread, standardized sharing mechanism among Patient Education teams. References: Palumbo, R. Examining the impacts of health literacy on healthcare costs. Health Services Management Research. 2017. 30(4): p. 197-212. Stenberg, U., et al., Health economic evaluations of patient education interventions a scoping review of the literature. Patient Education and Counseling, 2018. 101(6): p. 1006-1035.

3B-3: Intervention suggestions to improve uptake of breast, cervical, and colorectal cancer screening among patients living with serious mental illness

Aleigha Binda ${ }^{1}$, Janna Gordon ${ }^{1}$, Elizabeth Alpert ${ }^{1}$, Kristina Greenwood ${ }^{2}$, Rosa Cobian Aguilar ${ }^{1}$, Caroline Atterton ${ }^{2}$, Cristian Garcia-Alcaraz ${ }^{1}$, Nicholas Lucido ${ }^{1}$, Vanessa Arellano ${ }^{1}$, Michael Plopper ${ }^{3}$, Kristen Wells ${ }^{1}$ ${ }^{1}$ UCSD Moores Cancer Center, La Jolla, CA, USA, ${ }^{2}$ Sharp HealthCare Outcomes Research Institute, San Diego, CA, USA

Abstract: Individuals living with serious mental illness (SMI) have a higher risk of dying from cancer and are less likely to engage in recommended cancer screening. This study examined the perspective of individuals living with SMI and their mental health care providers regarding the design of a cancer screening promotion intervention. This study used Intervention Mapping guided by the Social Ecological Model (SEM). Purposeful sampling yielded 25 people with SMI (mean age: 71.4 years; $60 \%$ female) and 15 stakeholders (mean age: 45.3 years; $80 \%$ female), who provide mental health services to people with SMI in San Diego, California. Participants completed semi-structured in-depth interviews to assess the needs and assets of a potential intervention to facilitate adherence to recommended breast, cervical, and colorectal cancer screening. Interviews were recorded, transcribed verbatim by research staff, and imported into NVivo. Content analysis and the constant comparison method were used to analyze interview data. Participants suggested various individual, interpersonal, and community-wide intervention strategies, including educating patients about screening and the importance of screening, providing a reassuring and comforting environment, and providing assistance for coping with exacerbation of mental health symptoms during screening. Participants most commonly recommended mental health providers, medical providers, family members, friends, and other caregivers to provide cancer screening information. A wide range of places were suggested for the provision of the intervention, the most common place being a health care provider's office or providing the intervention via various media outlets, with TV ads being the most commonly suggested. Cancer screening interventions should be delivered in a reassuring way to people living with SMI to facilitate coping with distress related to the screening experience. Study findings could be used to develop interventions that can potentially improve uptake of breast, cervical, and colorectal cancer screening among individuals living with SMI. Objectives: The audience will be able to identify intervention preferences as suggested by individuals living with SMI and their care providers to create an intervention to improve and facilitate uptake of breast, cervical, colorectal cancer screening. References: Happell B, Scott D, Platania-Phung C. Provision of preventive services for cancer and infectious diseases among individuals with serious mental illness. Archives of psychiatric nursing. 2012;26(3):192-201. Howard LM, Barley EA, Davies E, et al. Cancer diagnosis in people with severe mental illness: practical and ethical issues. The lancet oncology. 2010;11(8):797-804.

\section{B-4: Interdisciplinary Program Development and Evaluation: HCT Discharge Class for Caregivers Focused on Infection Prevention and Self-care}

Dhruti Ramchandani, Ranee Kang, Kathleen Kelly, Jackelene Valdez, Gabriela Flores, Artis Rivera

City of Hope, Duarte, CA, USA

Abstract: Caregivers experience significant stress, especially caring for the Hematopoietic Cell Transplant (HCT) patient. Many hospitals require a caregiver to be with a patient post-transplant. A focus on caregivers is critical because their distress can match or surpass the distress levels of the HCT patient. Caregivers report a lack of support and skills in self-care and higher rates of depression and anxiety. This negatively impacts caregivers' physical and mental health and the patient's recovery. An interdisciplinary team (health educator, RNs, CSWs, MDs, dietitians, and other consultative members) developed a class that is co-lead by an RN and CSW. The CSW provides education on self-care practices, sexuality, relationship adjustments, and caregiver resources. The RN discussed the home environment, pets, socializing, food safety, nutrition, infection precautions, medication management, daily health assessment, graft versus host disease, and communicating with the medical team. PowerPoint presentations were supported by print materials, caregiver toolkit, and open discussion of caregiver concerns. The class evaluation assessed caregiver readiness for the care for HCT patient at home and self-care awareness. Among 48 caregivers, ages 18-65+; $71 \%$ female and $43 \%$ caring for a spouse/partner. Pre and post evaluations were analyzed using a paired t-test. Using a $0-10$ point Likert scale, caregiver readiness to care for the patient was significantly higher in the postevaluation $8.63+2.21$ than the pre-evaluation $6.81+2.83$, t $48=-3.50$, $\mathrm{p}$ pre-evaluation $7.33+2.48, \mathrm{t} 48=-3.21, \mathrm{p}<0.05$. Findings from this evaluation study suggest that this would be feasible and scalable to other centers. These findings support the integration of an inperson educational class as an effective means to support the confidence and well-being of caregivers of HCT patients. Objectives: 1 . The participant will be able to identify the two primary components of the HCT Discharge class that prepares caregivers to take home an HCT patient. 2. The participant will be able to list at least two benefits of the interdisciplinary approach between clinical social work, nursing, health educators, and a multidisciplinary team to provide accurate information at appropriate levels of health literacy, and effective delivery of self-care and infection prevention education. 3 . The participant will be able to share at least two reasons based on caregiver research to support educational program development for caregivers. References: Kareem Jamani, Lynn E. Onstad, Merav Bar, Paul A. Carpenter, Elizabeth F. Krakow, Rachel B. Salit, Mary E.D. Flowers, Stephanie J. Lee. Quality of Life of Caregivers of Hematopoietic Cell Transplant Recipients, Biology of Blood and Marrow Transplantation, Volume 24, Issue 11, 2018, Pages 22712276, ISSN 1083-8791, https://doi.org/10.1016/j.bbmt.2018.06.015. Bartolomei, Sonja et al. Transplant Discharge Education: Technology Versus People. Biology of Blood and Marrow Transplantation, Volume 25, Issue 3, S441, March 01, 2019. 
3B-5: Patient satisfaction of the mychoice ${ }^{\mathrm{TM}}$ tool to improve informed decision making regarding clinical trial participation

Cassidy Kenny ${ }^{1}$, Linda Fleisher ${ }^{1}$, Sarah Bass ${ }^{2}$, Jingwei $\mathrm{Wu}^{2}$

${ }^{1}$ Fox Chase Cancer Center, Philadelphia, PA, USA, ${ }^{2}$ Temple University, Philadelphia, PA, USA

Abstract: Mychoice ${ }^{\mathrm{TM}}$ is a web-enabled communication tool designed to improve informed decision making about clinical trials, especially among underrepresented racial/ethnic groups. A randomized control trial (RCT) was conducted at 4 cancer centers and tested the impact of the tool on patients' perceptions of self-efficacy, activation, knowledge, and decisional conflict specifically among white/non-white cancer patients. The mychoice $^{\mathrm{TM}}$ tool was developed using a mixed methods approach, including interviews, surveys, perceptual mapping analysis, and user prototype testing, resulting in an interactive communication aid which was evaluated in a multi-site RCT. Participants completed a baseline survey, were randomized to either mychoice tool or standard materials on clinical trials. Immediately upon using the tool, patients were asked to provide feedback on its decision making value (Ottawa Preparation for Decision Making Scale-PREP-DM). Impact on self-efficacy, knowledge, patient activation and satisfaction were also measured at one-month follow-up. Descriptive and bivariate analyses were conducted comparing the experimental and control groups. Participants $(\mathrm{N}=253)$ were diverse (39\% African American, $63 \%$ female, $30 \%$ less than a HS education). Almost all $(92 \%)$ who received mychoice ${ }^{\mathrm{TM}}$ indicated it was somewhat/very helpful and would recommend it (95\%). African Americans stated it helped them recognize that a decision needs to be made (83\%), and it prepared them to make a better decision $(88 \%)$. Most stated it helped them to personalize the pro's and con's $(82 \%)$, organize their thoughts (90\%), think about their involvement in decision making (93\%) and identify questions for their doctor $(86 \%)$. Similar results were seen in the total sample and with no racial differences. Rigorous formative evaluation using mixed method approaches ensured the salience to culturally diverse patients. Preliminary satisfaction results indicate that the mychoice ${ }^{\mathrm{TM}}$ communication tool was well received by patients in our RCT. Future plans include broader dissemination of the tool. Objectives: The participant shall be able to identify two attitudes related to satisfaction of the mychoice tool among black and non-black cancer patients at two leading cancer centers in Philadelphia. The participant shall be able to identify two barriers to informed decision making in minority populations. The participant shall be able to discuss how digital health can be used to promote informed decision making. References: Kessel KA, Vogel MM, Kessel C, et al. Mobile Health in Oncology: A Patient Survey About App-Assisted Cancer Care. JMIR Mhealth Uhealth. 2017;5(6):e81. Published 2017 Jun 14. doi:10.2196/mhealth.7689. Kim H, Goldsmith J, Sengupta S, Mahmood, Powell P, Bhatt J, Chang C, Bhuyan S. (2017). Mobile Health Application and e-Health Literacy: Opportunities and Concerns for Cancer Patients and Caregivers. Journal of Cancer Education. 10.1007/s13187-017-1293-5.

\section{C-1: Aberrant epigenetic alterations among African Americans: a review of their contribution to cancer disparities}

Andres Espinoza ${ }^{1}$, Georgia Robins Sadler ${ }^{1}$, France Nguyen-Grozavu ${ }^{1}$, Sandra Leibel ${ }^{1}$, Rachael McVicar ${ }^{2}$, Evan Snyder ${ }^{2}$

${ }^{1}$ UCSD Moores Cancer Center, La Jolla, CA, USA, ${ }^{2}$ Sanford Burnham Prebys Medical Discovery Institute, La Jolla, CA, USA

Abstract: African Americans (AA) experience the highest cancer mortality rates for most cancers. Studies indicate epigenetic modifications impact cancer progression and survival. This narrative literature review aims to identify differences in epigenomic profile between African American and Non-Hispanic White (NHW) cancer patients and potential causes for discrepancies in epigenomic pattern. This literature review used the databases PubMed, PsycINFO, CINAHL, and Google Scholar to search for evidence of epigenetic modifications among AA in association to cancer development and outcomes. Eligible articles were published between 2008 and 2020 and available as full-texts. The reference lists of the identified articles were also reviewed for additional eligible articles. The following key search terms were included in the review: African American, Black, cancer, disparities, DNA methylation, epigenetics, ethnic, histone modification, miRNA, and racial. While the scientific literature identified specific epigenetic alterations that promote oncogenic expression among AA, only a few articles explored these differences in comparison to other ethnic groups. Some studies revealed AA expressed higher frequency of DNA methylation abnormalities of prostate and breast cancer patients than NHW. Genes TRPC5, S100A14, and MIR662 were prevalently expressed among AA cancer patients due to hypomethylation and were associated with chemotherapy resistance. Evidence shows lifestyle factors such as diet, stress, smoking levels, alcohol consumption, and environmental toxin exposure change the epigenomic conformation. These long-term stressors can contribute to burdens in AA health outcomes, including cancer. AA experience high epigenetic abnormalities in genes associated with some cancers. Biological, socioeconomic, or environmental conditions may affect the methylation status of the genome, leading to the observed cancer mortality disparities among AA. This research also confirmed the critical need for more racial and ethnic diversity in epigenetic studies. Objectives: The participant shall be able to identify cancer types associated with prevalent methylation abnormalities among African American cancer patients. The participant shall also be able to identify socioeconomic and lifestyle factors that change the epigenetic conformation and could potentially promote oncogenic expression References: Lara, O. D., Wang, Y., Asare, A., Xu, T., Chiu, H.-S., Liu, Y., Hu, W., Sumazin, P., Uppal, S., Zhang, L., Rauh-Hain, J. A., \& Sood, A. K. (2020). Pan-cancer clinical and molecular analysis of racial disparities. Cancer, 126(4), 800-807. https://doi.org/10.1002/cncr.32598. Watson, K. S., Hulbert, A., Henderson, V., Chukwudozie, I. B., Aponte-Soto, L., Lerner, L., Martinez, E., Kim, S., \& Winn, R. A. (2019). Lung Cancer Screening and Epigenetics in African Americans: The Role of the Socioecological Framework. Frontiers in Oncology, 9, 87. https://doi.org/10.3389/fonc. 2019.00087.

3C-2: Biobehavioral correlates of high-grade, precancerous cervical lesions and invasive cervical cancer among women living with HIV: A Systematic Review

Olufunto Olusanya ${ }^{1}$, Aishatu Yusuf ${ }^{1}$, Aditi Tomar $^{1}$, Ibraheem Karaye $^{1}$, Onoriode Kesiena ${ }^{1}$, Jessica Wells ${ }^{2}$, Lisa Tisdale Wigfall ${ }^{1}$

${ }^{1}$ Texas A\&M University, College Station, TX, USA, ${ }^{2}$ Emory University, School of Nursing, Atlanta, GA, USA

Abstract: Cervical cancer is the fourth most prevalent malignancy among women globally. Immunosuppression from human immunodeficiency virus (HIV) is associated with persistence of human papillomavirus (HPV) infection, and progression to high-grade cervical lesions (cervical intraepithelial neoplasia $2+[\mathrm{CIN} 2+])$. We systematically reviewed literature to describe virologic, behavioral, and sociodemographic correlates of CIN2+ among women living with HIV (WLHIV). Using the Preferred Reporting Items for Systematic Reviews and Meta-Analyses (PRISMA) guidelines, articles published from January 2014 till October 2019 were searched in MEDLINE, CINAHL, Cochrane CENTRAL, and EMBASE electronic databases. Inclusion criteria included peer-reviewed, original studies focused on examining the predictors for CIN2+ among WLHIV ages 18 to 64 years. The electronic database search generated 583 de-duplicated articles. Preliminary screening of 100 titles/abstracts and 41 full-text articles yielded 16 studies which met eligibility criteria for inclusion in the analysis. Selected articles were collectively appraised by three reviewers (A.T.,A.Y.,\&O.O.) in two independent screenings. Studies' methodological quality was assessed using the NIH Quality Assessment Tool for Observational Cohort and Cross-Sectional Studies. 
WLHIV were significantly more likely to have CIN2+ if they were $=200$ cells $/ \mathrm{mm} 3$ were $43 \%(\mathrm{p}=0.001)$ and $19 \%(\mathrm{p}=0.002)$ associated with lower risk of progression to CIN2+, respectively. Preliminary findings depict disproportionate impacts of CIN2+ on WLHIV due to HPV/HIV coinfection and inform efforts to reduce cervical cancer disparities. Population-based interventions and community partnerships which address risk factors for $\mathrm{CIN} 2+$, as well as, mitigate barriers to cervical cancer prevention services i.e., HPV testing, quadrivalent/bivalent HPV vaccine are warranted among high-risk, susceptible populations. Objectives: The participant shall be able to identify biological and behavioral factors associated with the progression to precancerous cervical lesions and invasive cervical cancer among WLHIV. References: 1. Kelly, H. A., Ngou, J., Chikandiwa, A., Sawadogo, B., Gilham, C., Omar, T., ... \& Delany-Moretlwe, S. (2017). Associations of Human Papillomavirus (HPV) genotypes with high-grade cervical neoplasia (CIN2+) in a cohort of women living with HIV in Burkina Faso and South Africa. PloS one, 12(3). 2. Wang, Q., Ma, X., Zhang, X., Ong, J. J., Jing, J., Zhang, L., \& Wang, L. H. (2019). Human papillomavirus infection and associated factors for cervical intraepithelial neoplasia in women living with HIV in China: a cross-sectional study. Sexually transmitted infections, 95(2), 140-144

\section{C-3: Immune Regulation of Breast Tumor Progression and Metastasis}

Ryan Park, Jing Yang

UCSD Moores Cancer Center, La Jolla, CA, USA

Abstract: African American women have higher proportions of aggressive breast cancer subtypes and breast cancer mortality. The components of why specific cancers are therapeutically unresponsive is difficult to determine and for patients to understand. Thus it is important to understand how immune components of the tumor microenvironment (TME) regulate tumor progression. In order to elucidate how the specific immune cell, the CD8+ T cell, regulates cancer progression, we analyze changes in the cell RNA. We use a mouse model of HER2+ and triple negative breast cancer and track the tumor's progression. We then investigate the immune cell's effect on the tumor by isolating breast tumor cells and the nearby immune environment cells for RNA analysis between mice with and without this immune cell. We examine composition and tumor expression differences in their TME related to progression, anti-tumor response, and important oncogenic pathways using databases of important markers. We found that this specific type of immune cell, the CD8+ T cell, plays a major role in primary tumor cell initiation, proliferation, and metastasis. In addition, these cells regulate cell state and RNA changes in the TME leading to increased tumor cell metastasis. The interactions between cancer cells, CD8+ T cells, and nearby immune cells can ultimately result in an environment that fosters tumor growth and metastasis. Understanding the mechanisms underlying the relationship between cancer and immune cells will allow for further research into novel immunotherapeutics and understanding of cancer prognosis factors. Better knowledge of these mechanisms improves clinician and patient understanding of the factors leading to their specific outcomes and therapeutics that target multiple TME components. Objectives: The participant will be able to understand how the immune system regulates cancer progression and metastasis which can help in treating aggressive triple negative breast cancers that disproportionately affect African American women. The participant will also learn how to use scientific cancer research to educate audiences with varying levels of scientific and medical expertise. References: Binnewies $M$, et al. Understanding the tumor immune microenvironment (TIME) for effective therapy. Nat Med. 2018;24:541-550. doi: 10.1038/241591-0180014-x. Lawson DA, et al. Tumour heterogeneity and metastasis at single-cell resolution. Nat. Cell Biol. 2018;20:1349-1360. doi: 10.1038/s41556-018-0236-7.
3C-4: Pesticide exposure: A risk factor for childhood leukemia among Hispanic Americans

Eduardo Gonzalez, Briana Chronister, France Nguyen-Grozavu, Aleigha Binda, Jose R. Suarez-Lopez, Georgia Robins Sadler

UCSD Moores Cancer Center, La Jolla, CA, USA

Abstract: Whitehead and colleagues' 2016 study showed that from 1988 to 2012 childhood leukemia in California increased among Latino children, more than all races combined and Non-Latino Whites. We hypothesize that the evidence linking pesticide exposure to childhood leukemia is sufficient to warrant mandates to limit their pesticide exposure. A literature search for articles of childhood acute lymphocytic leukemia (ALL) or acute myeloid leukemia (AML) and pesticide exposure among Hispanics was conducted using PubMed and Google Scholar. Eligible articles were full-text accessible, written in English, and published since 2015. Eligible articles included systematic review articles, meta- and pooled analyses, and observational studies. The keywords used included combinations and variations of pesticides, cancer, agriculture, leukemia, Hispanic, Latino, children, adolescents, and exposure. Citation lists of eligible articles were reviewed to identify other eligible articles and search terms. Websites of reputable organizations were also searched for relevant information. Twelve out of fourteen articles linked pesticide exposure to childhood leukemia. Children of Latino fathers who were exposed to organic solvents had an increased risk for ALL (OR: 1.48 [95\% CI: $1.01,2.16])$. A Costa Rica study found maternal exposure to insecticides during breastfeeding a risk to ALL in boys $(1.75$ [1.12, 2.73]). Metaanalyses showed a risk for ALL associated with any pesticide exposure prenatally, during pregnancy, and postnatally were $1.39[1.25,1.55], 1.43$ $[1.32,1.54]$, and $1.36[1.23,1.51]$, respectively. For AML the associated risk was 1.49 [1.02, 2.16], 1.55 [1.21,1.99], and 1.08 [0.76, 1.53]. High rates of childhood leukemia in Hispanics are possibly due to primary, secondary, and tertiary exposure to toxic agricultural chemicals. Reducing exposure to pesticides in these communities can be achieved by advocating for occupational personal protective equipment (PPE) mandates and guidelines, and by teaching workers the proper use of PPE. Objectives: The participant shall be able to discuss disparities in childhood leukemia rates. The participant shall be able to identify at least three potential causes of childhood leukemia disparities. The participant shall be able to discuss at least two possible strategies for reducing those disparities. References: Bailey HD, Infante-Rivard C, Metayer C, et al. Home pesticide exposures and risk of childhood leukemia: Findings from the childhood leukemia international consortium. Int J Cancer. 2015;137(11):2644-2663. doi:10.1002/ijc.29631. Metayer C, Scelo G, Kang AY, et al. A task-based assessment of parental occupational exposure to organic solvents and other compounds and the risk of childhood leukemia in California. Environ Res. 2016;151:174-183. doi:10.1016/ j.envres.2016.06.047. Schüz J, Erdmann F. Environmental Exposure and Risk of Childhood Leukemia: An Overview. Arch Med Res. 2016;47(8):607-614. doi:10.1016/j.arcmed.2016.11.017. Whitehead TP, Metayer C, Wiemels JL, Singer AW, Miller MD. Childhood Leukemia and Primary Prevention. Curr Probl Pediatr Adolesc Health Care. 2016;46(10):317-352. doi:10.1016/j.cppeds.2016.08.004

\section{C-5: Developing Educational Materials for Patients Entering CAR T Cell Therapy and their Caregivers}

Claire Saxton ${ }^{1}$, Susan Ash Lee ${ }^{1}$, Kirstin Fearnley ${ }^{1}$, Elizabeth Franklin ${ }^{1}$, Maria Gonzalo ${ }^{1}$, Jenny Karubian ${ }^{2}$, Richa Ruwala ${ }^{1}$, Alex Swales ${ }^{1}$, Alexandra Zaleta ${ }^{3}$

${ }^{1}$ Cancer Support Community, Washington, DC, USA, ${ }^{2}$ Ready to Launch Research, Los Angeles, CA, USA, ${ }^{3}$ Cancer Support Community, Philadelphia, PA, USA

Abstract: To inform the development of educational materials for patients and caregivers facing CAR T therapy, Cancer Support Community 
(CSC) explored the challenges faced by CAR T patients and their caregivers, attempting to capture educational, logistical, and support needs for these patients and caregivers. CSC conducted a qualitative needs assessment, consisting of 14 in person and telephone interviews with a total of 5 patients, 3 caregivers and 11 HCPs at 8 institutions administering CAR T therapy. The interview questions were designed to identify the educational, support, and logistical needs of patients and caregivers at key milestones in the patient experience. The interviews also captured variations in administration across multiple institutions offering CAR T and insights from HCPs, patients, and caregivers about what resources would be most useful to those facing CAR T therapy. Findings from this analysis illustrated targeted areas of patient and caregiver unmet needs. In particular: Patients highlighted the need for print materials that explain the CAR T treatment process and the most common side effects. Caregivers emphasized the need for education about the severity of side effects, and how to respond effectively. While HCPs indicated the importance of developing concise materials that explain how CAR T works and give clear instructions for caregivers. As well as a list of existing financial resources to help pay for treatment and ancillary costs. CSC\&rsquo;s needs assessment indicate that for both patients and providers, treatment-related side effects are top of mind. This underscores the importance of developing turnkey education, resources, and tools to help patients and caregivers understand and manage CAR T-related side effects. Objectives: Understand the informational needs of patients and caregivers facing CAR $T$ therapy. Identify the specific challenges faced by CAR T patients and their caregivers as well as health care provider, patient, and caregiver perceptions of needs. Identify three educational, logistical, and support needs for CAR patients and caregivers. References: McConville, H., Harvey, M., Callahan, C., Motley, L., Difilippo, H., \&amp; White, C. (2017). CAR Tcell therapy effects: Review of procedures and patient education [Online exclusive]. Clinical Journal of Oncology Nursing, 21(3), E79\&ndash;E86. https://doi.org/10.1188/17.CJON.E79-E86

\section{A-1: Development of a 360-degree, asynchronous, on-line under- graduate cancer curriculum in rapid response to the COVID-19 pan- demic}

Miklos Fogarasi, Miriam DiMaio, Jennifer Dreiling, Lynn Copes, Naveen Kumar Reddy, Courtney Mullen, Linda Durhan

Frank H Netter MD, School of Medicine, Quinnipiac University, Hamden, CT, USA

Abstract: The COVID-19 pandemic has posed unprecedented challenges in all aspects of life, including the provision of medical education. M4 students normally on hospital electives had to transition back to on-line courses created by pre-clinical faculty. Some of our students dispersed to out-of-state locations in different time zones or were in sub-optimal educational environments, while faculty faced the challenge of creating new educational materials under extreme time pressures. All this essentially created inequitable circumstances for learning. A $360^{\circ}$ clinical cancer course was rapidly developed utilizing our library's reference collection and our professional experiences. Task-based, multi-specialty integration facilitated critical thinking and constructivist learning. Adapting to a fluid COVID situation, students could receive 2-3-4 weeks of credit, by completing 6-9-12 modules. Course content included modules on cancer epidemiology, health disparities, cost/value-based care issue, clinical/ anatomical correlations, genetics, pathology, and imaging. After reviewing surgical, radiation and systemic therapy, learners studied AYA/elderly survivorship and end-of-life communication. In two sequential Spring 2020 blocks twelve Y4 students registered. Of the nine M4s enrolling for the course for full credit, one needed extra time to complete the course, one has earned only partial credit and one withdrew from class, due to major family challenges. Three students taking other classes in parallel, were all able to earn partial credit here. Surveying adequacy of time allocated (72\%), efficiency of on-line coursehub on Blackboard (90\%), task-based assignments (72\%), perceived improvement in knowledge (100\%) were all encouraging. Less than $20 \%$ favors a fully asynchronous design, with most students preferring a hybrid (live or zoom-based) construction. In summary, it was feasible to construct a multi-specialty cancer curriculum in rapid response to the COVID-19 pandemic. Delivered with flexible asynchronous design, we were able to mitigate pandemic-induced educational challenges serving our students in diverse settings of learning. The course may have utility in supplementing our pre-existing didactic cancer curriculum. Objectives: The participant shall be able to list three challenges in medical education experienced by learner (student) and faculty during the COVID-19 pandemic. The participant shall be able to describe five key components of a $360^{\circ}$, fully on-line clinical undergraduate cancer course, when designing/ updating their curriculum. The participant shall be able to recall at least five course-design elements used successfully to mitigate inequitable learning environments of students during the pandemic. References: 1/ Dittrich C, Kosty M, Jezdic S et al: ESMO/ASCO Recommendations for a Global Curriculum in Medical Oncology Edition 2016 ESMO Open 2016;1:e000097. doi:10.1136/esmoopen-2016- 000097. 2/ McRae RJ: Oncology Education in Medical Schools: Towards an Approach that Reflects Australia's Heath Care Needs. Journal of Cancer Education, 31, 621-625(2016) and also https://wiki.cancer.org.au/ oncologyformedicalstudents/Ideal_Oncology_Curriculum. 3/ Shapiro, CL; Jacobsen, PB; Henderson, T; Hurria, A. et al: ASCO Core Curriculum for Cancer Survivorship Education, J Onc Practice Feb 2016, 12(2); e108-e117, DOI: 10.1200/JOP.2015.009449.

\section{A-2: Democratizing statistics education using free interactive appli- cations}

Sujata Patil, Axel Martin, Jaya Satagopan

Memorial Sloan Kettering Cancer Center, New York, NY, USA

Abstract: Statistics literacy is crucial for pre-clinical laboratory researchers engaged in anticancer drug development. We developed a short course in statistics for biomedical researchers using didactic lectures and data examples from published research. Yet, foundational concepts often remain abstract to most researchers. Therefore, we developed computerbased apps that allow biomedical researchers to explore and visualize fundamental ideas at their own pace, thus empowering them to understand and use statistics in their research. We implemented interactive computer apps to train biomedical researchers in various concepts, including the central limit theorem, experimental designs, type I error, power, p-value, hypothesis testing, and multiple comparisons. Our apps, based on the R statistical programming language, facilitate inquiry-led activities to increase motivation and promote statistical thinking. Since $\mathrm{R}$ is a free-ware, our apps can be easily installed and used by interested colleagues from anywhere in the world, thus democratizing statistics education. We evaluated the computer apps using a pre-post format to assess how well participants were able to comprehend statistical concepts from the beginning to the end of the course. We included questions about the app in the post-course evaluation. There were two determinate questions: (i) Did the apps keep you adequately engaged?; and (ii) Did the apps help you learn the design and analysis concepts better? There was one open-ended question: Please provide suggestions on ways to improve the app. We will present the results of these evaluations and planned strategies for more effective future evaluation of the apps. By providing interactive learning that can be used via a freely available statistical software throughout the world, our apps have the potential for delivering equitable statistics education to all societies. Objectives: The audience will be able to list at least six statistical concepts that are amenable to interactive learning via computer apps. The audience will be able to download the $\mathrm{R}$ programming language and use our computer apps on their laptop for interactive statistics learning. References: Fawcett, L (2018). Using interactive Shiny applications to facilitate researchinformed learning and teaching. Journal of Statistics Education, 26(1):2-16. 
4A-3: Adapting an in-person summer internship program to a virtual format: Experience of a quantitative sciences undergraduate internship program during COVID-19

Kay See Tan, Mengmeng (Margaret) Du

Memorial Sloan Kettering Cancer Center, New York, NY, USA

Abstract: The Quantitative Sciences Undergraduate Research Experience (QSURE) is an in-person summer internship program designed to meet increasing demands for experiential learning. QSURE aims to enhance undergraduate students' knowledge of cancer and prompt interest in education and careers in cancer-related quantitative sciences. However, with mandatory closures of many organizations and "shelter-in-place" orders due to the COVID-19 pandemic, the summer 2020 program could not be completed on-site. Instead, a Virtual Internship Platform for QSURE (QSURE-VIP) was created. Key components of the 8-week program, such as the mentored cancer-related quantitative research project, were converted to a virtual platform facilitated by virtual collaboration and teleconference systems such as Microsoft Teams and Zoom. To ensure the virtual format is educational and effective, QSURE-VIP is designed to meet the following virtual internship guidelines: (1) defined learning goals, (2) thorough onboarding and orientation, (4) timely feedback, (5) consistent communication, (6) pre-arranged schedules, and (7) organizational involvement. Daily virtual group check-ins with the program directors allow students to share about the challenges they are facing. In addition, pre- and post-program quantitative evaluations and mid-project qualitative evaluations were developed and administered to elicit students' feedback on their readiness for and perceived success of the virtual format. Students indicated above-average baseline proficiency in communicating virtually, poor to moderate levels conducting team meetings online, using remote desktop for analyses, and managing work and productivity, and a desire to become highly proficient in all these skills. Post-program evaluations will be obtained shortly. We will present an analysis of all success measures. By adapting closely from the in-person internship program, QSURE-VIP can provide students with a safe, work-based collaborative learning experience, while developing their skills in online project management and communications. QSURE-VIP continues to inspire undergraduates to pursue graduate studies and seek career opportunities in quantitative methods in oncology and population sciences. Objectives: The participant will be able to identify the advantages and disadvantages of an online internship program. The participant will be able to describe the steps taken to adapt an in-person summer cancer-related quantitative research experience program into a virtual program and detail the guidelines to conducting an effective virtual program. The participant will be able to prepare a project assessment tool to evaluate the success of a virtual internship program. References: Fancher A. Virtual Internship Guidelines. University of Portland Career Center, https://www.up.edu/career/files/virtual-internships-guidelinesfor-employers.pdf. The Center for Research on College-Workforce Transitions. What to do about internships in light of the COVID-19 pandemic? A short guide to online internships for colleges, students, and employers. University of Wisconsin-Madison, 2020, http://ccwt. wceruw.org/documents/CCWT_report_COVID-19\%20Internships.pdf

\section{A-4: Éxito! Latino Cancer Research Leadership Training:} Motivating Latino students to achieve their ultimate success

Daniel Hughes ${ }^{1}$, Arely Perez ${ }^{1}$, Stacy Cantu-Pawlik ${ }^{2}$, Rena Pasick ${ }^{3}$, Amelie Ramirez

${ }^{1}$ University of Texas Health Science Center-Institute for Health Promotion Research, San Antonio, Texas, USA, ${ }^{2}$ University of Texas Health San Antonio, San Antonio, TX, USA, ${ }^{3}$ University of Calfornia, San Francisco, San Francisco, CA, USA

Abstract: The Latino cancer burden will rise to $142 \%$ by 2030 . Cancer is the leading cause of death among Latinos who represent $18 \%$ of the population and the majority minority population in the US. Yet, the lowest underrepresented group with a doctoral degree. This disproportionate representation and Latino health disparities is a multifaceted issue that includes cultural factors related to social determinants of health. This disparity requires increasing the pipeline of Latino health science researchers and professionals. Éxito! annually selects 25 master's-level participants to an intense five-day Summer Institute (SI). The interactive sessions promote collaboration between trainees, presenters, and mentors. Sessions are led by successful Latino leaders in a culturally congruent atmosphere that enhances academic self-efficacy in overcoming barriers, provides social support, networking and mentoring. To enhance research skills, competitive internships are awarded to 10 of the alumni of the SI working with a mentor focused on researching Latino health disparities. Program success is measured by changes in academic self-efficacy, e.g. "accepted into program of choice" ; confidence to overcome academic barriers, e.g. "applying to a program"; improvement in research skills (internships); and, successful acceptance into a doctoral degree program. Since 2010, 200 individuals have completed SI training and 49 internships have been awarded. Éxito! has improved academic self-efficacy (p improved confidence toward obtaining a doctoral degree ( $\mathrm{p}$ and improved research skills (p Of the 200 Éxito! alumni, 26\% (n=53) have successfully enrolled in doctoral programs; with, 12 receiving their doctoral degrees. Despite the successes to date, we are creating a multi-variate predictive model based on analyses from the alumni that have succeeded and not succeeded. The results will inform enhancements to our program as we seek funding to improve the Éxito! training program to further increase the research/health professional diversity pipeline. Objectives: The participant shall be able to : 1) Detail how the Éxito! program uses cultural congruence to enhance participant self-efficacy, confidence, research skills and confidence for Latino students desiring to continue their education focusing on Latino health disparities. 2) To describe the importance of cultural congruence between those that seek health care and those that research and provide that care. 3) To describe how the Éxito! model and results to date can be applied to other ethno-cultural under-represented groups. References: Tate, K. A., Fouad, N. A., Marks, L. R., Young, G., Guzman, E., \& Williams, E. G. (2015). Underrepresented first-generation, low-income colleague students' pursuing of a graduate Cancer education: investigating the influence of self-efficacy, coping efficacy, and family influence. J Career Assess, 23(3), 427-441. American Cancer Society. American Cancer Society. Cancer Facts \& Figures for Hispanics/Latinos 2018-2020. American Cancer Society. Rubio DM, Hamm ME, Mayowski CA, et al. Developing a Training Program to Diversify the Biomedical Research Workforce. Acad Med. 2019.

\section{A-5: Summer pediatric oncology education and research experi- ences for pre-doctoral biomedical science students}

Suzanne Gronemeyer, James Marmion, Gerard Zambetti St. Jude Children's Research Hospital, Memphis, TN, USA

Abstract: Our Pediatric Oncology Education (POE) Program http:// www.stjude.org/poe at St. Jude Children's Research Hospital is in its forty-third year. It is an NCI-funded (5R25CA023944-37) R25 summer research internship for pre-doctoral US students in the biomedical sciences. More than 500 students apply each year via CollegeNET. To date, 1,363 individuals have participated, 305 (22.4\%) of whom identified as under-represented minority (URM). Ongoing long-term follow-up utilizes web-based resources such as Google, LinkedIn, and Facebook. We focus on highly qualified applicants with a serious career interest in clinical or laboratory cancer research. Faculty mentors select applicants who share their research interests to participate in their ongoing research projects. Participants attend a daily cancer-focused Lunch \& Learn series designed specifically for them. All present their research at Lunch \& Learn. All submit a manuscript on their research written in the style of a journal in which their mentor publishes. Ongoing evaluation of the program is provided by pre- and post-experience testing of the student's 
knowledge of pediatric cancer and related areas, and by post-experience surveys completed by all students and mentors. Experienced cancer educators from prominent cancer centers review the program on site. We have follow-up for 1,354 (99.3\%) of 1,363 alumni. Of the 1,151 who have finished their academic degree work, $991(86.1 \%)$ hold a doctorate, including 211 (79.9\%) of the 264 URM in the cohort. Alumni include 55 $\mathrm{PhDs}, 29 \mathrm{MD} / \mathrm{PhDs}$, $5 \mathrm{PharmD} / \mathrm{PhDs}$, and some 800 physicians. Another 172 are currently in a doctoral program. Twenty-two (9 URM) have participated or are participating in the NIH IRTA or MRSP program. Alumni include 34 pediatric oncologists, 18 medical oncologists, 34 surgical, radiation, or gynecological/urological oncologists, 12 medical physicists, and 59 PharmDs. The 1188 alumni from years 1997-2019 are co-authors on some $440 \mathrm{St}$. Jude peer-reviewed publications. Objectives: The participant shall be able identify components of a successful long-running cancer education program, accomplishments and outcomes of a successful long-running cancer education program, reasons why knowledge gained about pediatric cancer is helpful to future physicians and their patients. References: Desmond RA, Venkatesh R, Padilla LA, Daniel CL, Litton AG, Heimburger DC, Michael Brooks C, Waterbor JW. Twenty-five-Year Follow-up of Short-term Cancer Research Trainees at the University of Alabama at Birmingham: a Brief Report. J Cancer Educ (2019) 34:1010-1013. Hein, DW, Kidd LR. Design and Success of a 21 st Century Cancer Education Program at the University of Louisville. J Cancer Educ (2018) 33:298-308. Korczak JF, Chung DW, Rosemond E, Von Hoff DD, Haspel RL, Waterbor JW, Chang S, Ramirez AG, Perkins S, Wiest J, Lei M. The National Cancer Institute R25 Cancer Education Grants Program: A Workshop Report. J Cancer Educ (2017) 32:3-10.

\section{B-1: Withdrawn}

\section{B-2: Does Race and Medical Mistrust matter in Breast Cancer} Survivors' Health Beliefs?

Megan Edmonds ${ }^{1}$, Bassam Dahman ${ }^{1}$, Sun Jung Kim ${ }^{1}$, Vanessa Sheppard ${ }^{2}$

${ }^{1}$ Virginia Commonwealth Univeristy, Richmond, VA, USA, ${ }^{2}$ Virginia Commonwealth University School of Medicine, Richmond, VA, USA

\begin{abstract}
Breast cancer beliefs are widely studied in women to improve mammography screening; however, limited research has examined breast cancer beliefs among breast cancer survivors. The purpose of this study was to investigate the influence of race and medical in survivors' beliefs in disease severity and susceptibility to a recurrence. This study is a secondary analysis of data from the Narrowing Gaps in Adjuvant Therapy Study (2006-2011), where Black $(\mathrm{N}=210)$ and White women $(\mathrm{N}=149)$ were interviewed 12 months following their breast cancer diagnosis and primary surgery in Washington D.C and Detroit, MI. Outcomes were perceived susceptibility to a recurrence and perceived severity of breast cancer. Bivariate analyses were conducted to assess racial differences in sample characteristics. Multiple regression analysis examined correlates between independent variables (e.g., sociodemographic/clinical) and women's total scores measuring breast cancer attitudes about susceptibility of a recurrence and disease severity. Most of the sample were Black women (58\%), diagnosed at Stage I $(46 \%)$, and privately insured (67\%). Black women reported higher perceived severity attitudes than White women. Higher perceived severity attitudes were associated with lower education, having public insurance and greater levels of medical mistrust. Women with later stage cancers vs. earlier-stage had significantly greater perceived susceptibility to a recurrence. In our adjusted multiple regression model women with lower satisfaction with financial aspects of their healthcare were more likely to have higher perceived severity attitudes. Survivors' appraisals of their breast cancer may, in part serve as adherence determinants to follow-up care regimens (e.g., surveillance mammography). Results concluded Black-White differences and identified subgroups to target in future
\end{abstract}

studies. Improved understanding of survivors' health beliefs informs navigation strategies in the context of psycho-oncology and follow-up cancer care. Objectives: 1)Participants will be able to identify different social determinant factors relation to breast health beliefs among breast cancer survivors. 2)Participants will be able to identify important subgroups of survivors for opportunities to intervene. 3)Participants will also be able to identify steps in applying a theoretical framework as a guide in research. Additionally, participants will identify at least two implications from this study. References: Miller AM, Champion VL. Attitudes about breast cancer and mammography: racial, income, and educational differences. Women Health. 1997;26: 41-63. Rutledge DN, Hartmann WH, Kinman PO, Winfield AC. Exploration of factors affecting mammography behaviors. Prev Med. 1988;17: 412-422.

\section{B-3: Adult Cancer Survivorship eLearning: Adapting high quality} nursing education for patients and families

Karen Lawrie ${ }^{1}$, Janet Papadakos ${ }^{1}$, Nicole Liscio ${ }^{1}$, Christine (Tina) Papadakos $^{2}$

${ }^{1}$ Princess Margaret Cancer Centre, Toronto, ON, Canada, ${ }^{2}$ University Health Network, Toronto, ON, Canada

Abstract: Cancer survivorship in Canada is expected to increase by about $70 \%$ in the next two years. 1 Despite this, there are limited resources to support people post-treatment. The Cancer Education program at the Princess Margaret Cancer Centre partnered with the Canadian Association of Nurses in Oncology (CANO) to translate updated nursing education on survivorship into a series of eLearning modules to provide patient and caregivers with relevant, timely information to prepare for life after cancer treatment. Using updated nursing education on survivorship by CANO, five patient/caregiver eLearning modules were created following patient education best practices. Two plain language specialists reviewed the CANO education modules to extract the most salient information based on the $3 \mathrm{Ws}$ and an $\mathrm{H}$ framework for developing quality patient education resources. 2 Throughout the process, subject matter experts and authors of the CANO resources were consulted to ensure accuracy of the plain language review and content relevancy. In order to monitor the impact of the eLearning series, a number of indictors are being monitored including the number of views, geolocation of users and at the end of each eLearning module, there is an embedded survey to ensure ongoing QI evaluation is in place in order to monitor the quality and acceptability of the modules. The survey consists of a series of questions to measure patient and caregiver satisfaction and to collect any new questions that may have emerged as a result of the module. On average, it takes an estimated 17 years for research to be adopted into practice. 3 Employing knowledge translation strategies to adapt current nursing education to patient content, as quickly as appropriate, ensures that patients benefit from advances in clinical knowledge without unnecessary delays. Objectives: The participant will learn and see examples of how complex clinical information can be conveyed to patients/caregivers in a timely manner that allows for better comprehension, and increased ability to self-manage post-treatment needs. References: 1) Canadian Cancer Statistics Advisory Committee. Canadian Cancer Statistics 2019. Toronto, ON: Canadian Cancer Society; 2019. Available at: cancer.ca/Canadian-Cancer-Statistics2019-EN (accessed 31 August, 2020). 2) Papadakos CT, Papadakos J, Catton P, Houston P, McKernan P, Jusko Friedman A. From theory to pamphlet: the $3 \mathrm{Ws}$ and an $\mathrm{H}$ process for the development of meaningful patient education resources. J Cancer Educ. 2014;29(2):304310. doi:10.1007/s13187-013-0600-z. 3) Morris ZS, Wooding S, Grant J. The answer is 17 years, what is the question: understanding time lags in translational research. J R Soc Med. 2011;104(12):510520. doi:10.1258/jrsm.2011.110180 
4B-4: Blending Cancer Prevention Science and Professional Development: Communication Skills for Scientists-in-training

\section{Lotis Ann Batan, Maria Monberg Monberg, Shine Chang} UT MD Anderson, Houston, TX, USA

\begin{abstract}
For long-term success, trainees preparing for careers in research must be competent in multiple modes of communication - written, rehearsed presentation, and spontaneous dialogue. Mastery of these modes has always been important for career advancement, but trainees do not always get opportunities for formal instruction and practice. Further, in the 21st century, new formats for communication have entered the cannon of communication needed by scientists, including the popular TED talk. The semester graduate course, "Bio-Behavioral Research Methods in Cancer Prevention and Addiction," prepares students for team science by showcasing cross-disciplinary collaborative research. Students practice introducing speakers and facilitating Q\&A sessions, and explore the roles of communication in career advancement, including for social support from non-scientist family and friends. Instruction in giving TED talks is led by a course co-coordinator with four years experience as a speaker team lead for a Big10 TEDx initiative. For each 2-hour session, evaluations were conducted for speakers and for students assigned to introduce speakers and facilitate. Students were often eager to improve their skills based on ratings and written feedback. Engagement activities were also evaluated for effectiveness as were the course coordinators. The culminating course activity was a TED talk presentation by each student, building upon feedback from all classmates provided in a "round-robin" format and in individual coaching. Finalists for live presentation and a "People's Choice Award" were selected from classmate votes. A winner was selected using predefined criteria by knowledgeable guest judges. Over one semester, students practiced a variety of communication modes, from small to large stakes. The simultaneous emphasis on multiple communication skills was self-reinforcing. Creating TED Talks is an excellent career tool to develop communication and engagement skills across disciplines, form relationships, gain support for ideas, and build professional confidence. Objectives: Initially, the participant shall be taught the nature and goals of TED talks as a communication tool. Through courses and interactive feedback sessions, the participant will be trained to transform their own research objectives into a TED-style talk. To evaluate the efficacy of the TED-style talk training program, the participants will engage in a "talk competition", wherein they will present their talks to Public Speaking judges who will assess the ability of each student to deliver a meaningful talk that adequately describes the focus of their research (as it pertains to the social determinants of health and cancer education) and presents a solution to the issues presented. References: Andersen C. TED TALKS, The official TED Guide to Public Speaking. Boston: Mariner Books; 2017
\end{abstract}

\section{B-5: Diamond Anniversary for the AACE}

George Hill

Hilltree Farm Press, Baltimore, MD, USA

\begin{abstract}
The American Association for Cancer Education will be seventy-five years old in 2021. This paper will discuss some of the highlights of the organization, with a concluding statement of its unique place in health care organizations. The AACE was founded as the Coordinators of Cancer Teachers in 1946, when a group of surgeons met to discuss how best to teach the subject of cancer to medical students. Dr. Samuel Clark Harvey (1886-1953) accepted the leadership of the organization that they formed. In the forty years between 1946 and 1986 many discoveries were made of the cause of cancer and of new methods to treat the disease. Other branches of medicine developed special interests in cancer, and each was represented in the education of students of the health professions. The original leaders specialized in surgery, pathology, radiation, internal medicine, and hematology. Oncology specialties developed in
\end{abstract}

many diverse fields, and they were represented in the leadership of the AACE. The new fields included medical oncology, radiation oncology, surgical and urological oncology, pediatric oncology, gynecological oncology, and preventive oncology. The AACE developed close relationships with medical schools, Cancer Centers, National Cancer Institute, American Cancer Society, American College of Surgeons, the American Society of Clinical Oncology, and the Oncology Nursing Society. By the mid-1980s, the AACE had begun to build bridges to other professions that were involved in health care. The AACE welcomed leaders from dentistry, nursing, rehabilitation, laboratory research, and public education, as well as cancer specialists in Canada, Europe, and elsewhere in the world. Objectives: Members of the AACE can be proud of what their organization has achieved. There is arguably no other organization in the field of health care that at this time has a broader base of members and leaders than the American Association for Cancer Education. References: https://www.aaceonline.com/AACE/About Us/ Awards_Pages/Samuel_Harvey_Lecturers.aspx. Hill GJ. The American Association for Cancer Education: 40 Years of civic engineering in cancer. President's remarks to the AACE, November 12, 1986, Montreal, Quebec, Canada. J Canc Educ. 1987;2:83-8. Bakemeier RF, Kupchella CE, Chamberlain RM, Gallagher RE, O'Donnell JF, Parker JA, Hill GJ, Brooks CM. Cancer education in United States medical schools: Cancer Education Survey II - Survey design and observations relating to cancer education funding. J Cancer Educ 7(2):85-93, 1992. Hill GJ. In Memoriam: Margaret Hay Edwards, MD, MPH (1914-1994). J Cancer Ed. 1994;9:188-9.

\section{C-1: Development and Testing of an Adjuvant Radiotherapy Decision Aid for Older Women Diagnosed with Stage I Breast Cancer: A Pilot Study}

Ewa Szumacher ${ }^{1}$, Matthew Neve ${ }^{1}$, Nayanee Henry-Noel ${ }^{2}$, Rajin Mehta ${ }^{1}$, Maureen Trudeau ${ }^{2}$, Ines Menjak ${ }^{1}$

${ }^{1}$ Sunnybrook Health Sciences Centre, Toronto, ON, Canada, ${ }^{2}$ University of Toronto, Department of Medicine, Toronto, ON, Canada

Abstract: Develop and pilot a decision aid (DA), among women aged 65 years and older with Stage I breast cancer post breast conserving treatment, to examine its utility as a resource in increasing patient knowledge, lowering decisional conflict and increasing patient preparedness so they are fully informed about RT options post-lumpectomy. The DA was piloted among 40 patients 65 years and older post breast conserving treatment for low-risk breast cancer. The women completed a pre-DA Decisional Conflict Scale (DCS) and post-DA DCS, Knowledge, Preparation for Decision Making and Acceptability questionnaires. We then used descriptive statistics to analyse the results of the patient reported assessments and we compared the DCS scores pre- and post-DA utilization. The freamwork for development of this DA was the freamwork for development of decision aids form the Ottawa Research Institute(ORI) . We used step by step approach that has been suggested by the ORI. Median pre-DA DCS score was 31.2 (range 31.2-90.6) and median post-DA DCS score was 23.4 (range $0-75.0$ ). Of the 40 participants, six scored zero on the DCS after reviewing the DA and 13 participants scored less than 15.6. Median Knowledge score was 70.0\% (range 15.0-95.0\%) and median Preparation for Decision Making score was $90.0 \%$ (range 55.0-100\%). The Acceptability questionnaire demostrated high rates of satisfaction amongst participants with $99.0 \%$ of participants stating that the DA was clear and of potential value for future patients. Our findings demonstrated the utility of the DA as a resource for patients to understand their treatment options and in preparing patients to communicate with their radiation oncologist during their treatment decision-making process by lowering perceptions of uncertainty in deciding options and factors conductive to uncertainty. Objectives: The participant shall be able to identify at least three ways that Decision Aids may help a patient actively participate, to the extent they would like, in their treatment decisionmaking process. References: Correa, C., Harris, E. E., Leonardi, M. C., 
Smith, B. D., Taghian, A. G., Thompson, A. M., ... Harris, J. R. (2017). Accelerated partial breast irradiation: executive summary for the update of an ASTRO evidence-based consensus. Practical Radiation Oncology, 7(2), 73-79. Kunkler, I. H., Williams, L. J., Jack, W. J. L., Cameron, D. A., Dixon, J. M., \& PRIME II investigators. (2015). Breast-conserving surgery with or without irradiation in women aged 65 years or older with early breast cancer (PRIME II): a randomised controlled trial. Lancet Oncology, 16(3), 266-273. The Ottawa Hospital Research Institute, Patient decision aids: evaluation measures. (2019). Retrieved from https://decisionaid.ohri.ca/eval.html

\section{C-2: Caregiver skills training programs in cancer care: a scoping review}

Janet Papadakos, Diana Samoil, Rouhi Fazelzad, Ben Umakanthan, Rebecca Charow, Aman Sayal, Meredith Giuliani

Princess Margaret Cancer Centre, Toronto, ON, Canada

Abstract: In light of an increasing prevalence of cancer, an oncology workforce shortage, and a resource-stretched health system; oncology care is evolving towards the provision of complex treatment regimens in the outpatient setting. Patients' informal caregivers are therefore required to take on expanded care roles, subjecting them to burnout and distress. A scoping review was conducted to understand and summarize what is known about training programs for unpaid, family caregivers of cancer patients in the literature. The review was conducted using the Arksey \& O'Malley framework. A total of 10 databases were searched from January 2000 - May 2019. Of the 34,906 articles identified in the literature search, 119 met eligibility criteria. Articles were included in the review if they focused on a training program or educational intervention, had a target audience of unpaid adult caregivers (Age 18+), and were specific to the cancer context. Results indicate that existing training programs are predominantly focused on providing cancer caregivers with information on psychological well-being $(n=92,77 \%)$ and physical symptom management $(n=71,60 \%)$ to assist patients in the treatment $(n=66,55 \%)$ or palliative/end-of-life $(n=20,17 \%)$ stage of their disease. Most existing caregiver programs additionally require in-person attendance $(\mathrm{n}=83,70 \%)$ at a specific time, in a specific place. No comprehensive skills training programs were identified in the literature to holistically meet the needs of the cancer caregiver population. The cancer care system needs proactive and practical interventions to support caregivers, and skills training programs have demonstrated efficacy. The results of this review indicate an urgent need to create remotely accessible training programs for caregivers that are tailored beyond the psychological and physical aspects of cancer care. Objectives: 1 . The participant shall be able to identify at least two opportunities for improvement in the content and dissemination of cancer caregiver skills training programs. 2. The participant shall be able to understand the pressing need for the development of new remote implementation strategies for caregiver interventions. References: 1 . Kent EE., et al. Caring for caregivers and patients: research and clinical priorities for informal cancer caregiving. Cancer, 2016. 122(13): p.1987-95. 2. Wang, T., et al. Unmet care needs of advanced cancer patients and their informal caregivers: a systematic review. BMC Palliative Care, 2018. 17(1): p. 96.

4C-3: An inner-city smoking cessation program for adults with low socioeconomic status: a closer look at factors that predict successful tobacco cessation

Rachel Levine ${ }^{1}$, Siyu (Sylvia) Chen ${ }^{2}$, Sabrina Gonzalez ${ }^{1}$, Magnolia Contreras ${ }^{1}$

${ }^{1}$ Dana-Farber Cancer Institute, Boston, MA, USA, ${ }^{2}$ Tuft's University, Boston, MA, USA

Abstract: Dana-Farber Cancer Institute's education and counseling Tobacco Treatment Program (TTP) is offered at a Boston community health center and serves patients with extreme socioeconomic conditions and multiple co-morbidities. Given higher smoking rates among lowincome populations, this quality improvement study investigated factors predicting successful cessation in service of strengthening TTP. From 2015 to June 2019, 169 people participated in TTP. Data was analyzed for 105 participants with complete outcome and predictor variables. Based on self-report, forty-one individuals successfully quit their tobacco usage. Sixty-four individuals were not yet successful at quitting, defined as those who had not quit smoking and those who were in the process of quitting. Logistic regression was conducted with TTP success or not as the dependent variable and six predictor variables: current quit aid usage, spouse/partner smoking status, home smoking rules, alcohol consumption, having depression and/or anxiety, and number of TTP sessions attended. Three predictors were significantly related to the TTP outcome: spouse/partner smoking status, home smoking rules and number of TTP sessions. Participants whose spouse/partner also smoked had on average $66 \%$ lower odds of quitting compared to participants whose spouse/ partner did not smoke or did not have a spouse/partner (adjusted OR:0.34, 95\% CI:0.11-0.91, p-value:0.041). Participants who allowed smoking at home had on average 59\% lower odds of quitting, compared to participants who prohibited smoking at home (adjusted OR:0.41, 95\% CI:0.18-0.92, p-value:0.032). The odds of quitting increased on average $45 \%$ for each additional TTP session attended (adjusted OR:1.45, 95\% CI:1.10-1.95, p-value:0.011). Findings suggest that low-SES individuals would benefit from joint tobacco education with their spouse/partner and from smoke-free homes. The strong relationship between number of TTP sessions and cessation underscores the importance of ongoing TTP participation. Findings will be incorporated in outreach and educational materials to incentivize participation in these ways. Objectives: Participants should be able to identify two factors associated with difficulty in smoking cessation. Participants should be able to identify one factor associated wth successful smoking cessation. Participants should be able to articulate language to help encourage low SES individuals to enroll and continue participating in a smoking cessation intervention. References: CDC. Health Effects of Cigarette Smoking. [updated Jan_17_2018; accessed Mar 6 2019]. https://www.cdc.gov/tobacco/data statistics/ fact_sheets/health_effects/effects_cig_smoking/index.htm 2. CDC. Current Cigarette Smoking Among Adults in the United States. [updated Feb_4_2019; accessed Mar_6_2019]. https://www.cdc.gov/tobacco/ data_statistics/fact_sheets/adult_data/cig_smoking/index.htm. Stead LF et al. Combined pharmacotherapy and behavioural interventions for smoking cessation. Cochrane Database Syst Rev. 2016;3:CD008286. 4. Lüscher $\mathrm{J}$ et al. A daily diary study of joint quit attempts by dual-smoker couples: the role of received and provided social support. Nicotine Tob Res. 2017;20(1): 100-7. Kruger J et al. Smoke-free home and vehicle rules by tobacco use status among US adults. Prev Med [Internet]. 2015; 78:9-13 http://dx.doi.org/10.1016/j.ypmed.2015.06.004

\section{C-4: Group-Based Smoking Cessation Treatment Program for Incarcerated Smokers}

Pamela Valera ${ }^{1}$, Nicholas Acuna ${ }^{2}$, Ismary Vento ${ }^{2}$

${ }^{1}$ Rutgers, State University of New Jersey, Newark, NJ, USA, ${ }^{2}$ Rutgers University School of Public Health, Newark, NJ, USA

Abstract: Introduction. Group-based tobacco dependence treatment has been known to help smokers to quit in general adult populations, but the feasibility and efficacy of this type of smoking cessation treatment in correctional settings remain uncertain. Methods. A six-week group-based smoking cessation treatment with nicotine patches was implemented in seven prison facilities, in the Northeast, among male-incarcerated smokers. Exhaled breath carbon monoxide (CO) levels were collected from participants at each session to confirm smoking status. Participants were evaluated at the one-month follow-up to determine abstinence. Those who were lost to follow-up were recorded as continuing to smoke and not using nicotine patches. The goal of the study was to explore the 
feasibility and preliminary efficacy of conducting a smoking cessation treatment program for incarcerated smokers. Results. A total of 350 inmates were screened, 178 inmates were enrolled across the prison sites for the six-week program and 102 inmates completed the six-week program. Less than half $(21.3 \%)$ reported ever using electronic cigarettes at baseline. At Session 1, 116 individuals attended with a median CO level was 18.0 parts per million (ppm) and at the one-month follow-up there were 43 inmates who attended and had a median CO level of 5.00ppm. Discussion. Our study demonstrated preliminary efficacy and feasibility of group-based smoking cessation treatment with pharmacotherapy among incarcerated smokers. Objectives: The participant shall be able to describe how to implement group-based smoking cessation treatment in correctional settings. References: Djachenko, A., St John, W., \& Mitchell, C. (2015). Smoking cessation in male prisoners: a literature review. Int J Prison Health, 11(1), 39-48. doi:10.1108/IJPH-10-20140035

Kauffman, R. M., Ferketich, A. K., \& Wewers, M. E. (2008). Tobacco policy in American prisons, 2007. Tob Control, 17(5), 357-360. doi:10.1136/tc.2007.024448

\section{C-5: Developing and Implementing an Effective New Cancer Patient Resource}

Lauren Huff, Naa Kwarley Quartey, Nicole Liscio, Tina Papadakos, Janet Papadakos

Princess Margaret Cancer Centre, Toronto, ON, Canada

\begin{abstract}
The Cancer Journey resource was developed to engage patients in self-management. The first iteration was launched in the form of a binder. A formal evaluation ( $\mathrm{n}=228$ patients and caregivers) found that although highly valued, it was heavy and cumbersome to carry and participants reported that they had not received it as part of their care. In response, the binder was redesigned as a pair of light-weight books and a robust distribution plan was developed. Provision of the CJ resources was incorporated into nursing statements of work as an expectation of their role and we developed a digital ordering system to track inventory as a proxy for distribution statistics. The resources were then promoted through presentations to stakeholders to make all staff aware. Staff awareness was also raised through a social media marketing campaign, and posters were put up through the hospital to promote the resources to patients. Prior to print, we user-tested the CJ resources with five patients. Patients were given the books to read and use for two weeks. In-depth interviews were conducted to gather feedback. Patients suggested structural revisions and whole-heartedly endorsed the books. 5000 copies of each book were printed for a pilot launch. Evaluations with nursing staff show that they are easily able to integrate the provision of the CJ resources into patient care. Since launch there have been approximately 6,000 new patients to the cancer centre and between print and digital distribution (app downloads, webpage views), 8,731 have been provided to patients. The books provide patients with muchneeded information and an interactive medium to encourage engagement. This equips patients to take an active role in their care and can lead to the well-documented benefits of self-management. Through a carefully designed implementation strategy, these benefits are realized in a great number of patients. Objectives: Participants will learn about an approach to implementing a patient self-management tool across their organization. Participants will learn tips on how to improve satisfaction with their patient self-management tool. References: McCorkle R, Ercolano E, Lazenby M, et al. Self-management: Enabling and empowering patients living with cancer as a chronic illness. CA Cancer J Clin. 2011;61(1):50-62. doi:10.3322/ caac.20093. Bailo L, Guiddi P, Vergani L, Marton G, Pravettoni G. The patient perspective: investigating patient empowerment enablers and barriers within the oncological care process. Ecancermedicalscience. 2019;13:912. Published 2019 Mar 28.
\end{abstract} doi:10.3332/ecancer.2019.912

\section{Poster Presentations}

\section{P1: Disparities in the Cancer-Related Pain Experience}

Nicolette Olivia Le, Georgia Robins Sadler, France Nguyen-Grozavu UCSD Moores Cancer Center, La Jolla, CA, USA

Abstract: Pain is a feared companion of cancer. It influences people's beliefs and behaviors. It is known to deter adherence to screening guidelines, early evaluation of early warning signs, and initiation of/adherence to treatment. Cultural beliefs, which vary widely across racial and ethnic groups, can influence people's pain-related perceptions and beliefs This scientific literature review included articles that reported disparities in pain perception, measurement, and management across ethnic, racial, and cultural communities in the United States. Articles published from 2010 to 2020 that were accessible in English and examined pain perception related to any health issue among distinct demographic groups were eligible for inclusion. PubMed, GoogleScholar, and ScienceDirect databases were searched using variations of such terms as: pain experience, cancer pain, chronic pain, patient perceived barriers, professional-related barriers, pain assessment, and assessment instruments. Other terms were used in combination with those key words, such as, differences, disparities, race, culture, and ethnicity. Disparities in the pain experience were found to be well-documented among patients of African, Asian, Hispanic, Jewish, and Non-Hispanic White descent. However, authors identified inadequate pain assessment instruments, lack of instrument validation and norming across diverse communities, and wide variation of healthcare providers' pain management education as challenges to assessing pain-related disparities. In particular, the lack of medical guidelines related to prescribing opioids for cancer patients contributed to inconsistent practice patterns. Additionally, differences in patient care, pain self-reporting, and level of patient-provider trust based on sociodemographic characteristics and related historic disenfranchisement created inconsistencies in chronic oncological and non-oncological pain management. Effective pain management practices that take into account the influences of culture, ethnicity, and race, can help patients cope better with cancer, while also prompting changes in public opinion at the interface between cancer and pain. Culturally competent training programs and validated pain assessment instruments will also advance patient care. Objectives: The participant shall be able to identify at least two barriers to equitable cancer pain management. References: Crombez P, Bron D, Michiels S. Multicultural approaches of cancer pain. Curr Opin Oncol. 2019;31(4):268-274. doi:10.1097/CCO.0000000000000547. Krupić F, Čustović S, Jašarević M, et al. Ethnic differences in the perception of pain: a systematic review of qualitative and quantitative research. Med Glas (Zenica). 2019;16(1):108-114. doi:10.17392/966-19

\section{P2: Genetic Analysis of Mutation in cell cycle checkpoint gene:} Association with Breast Carcinoma

\section{Saima Saleem, Shiakh Umair Ali}

The Karachi Institute of Biotechnology and Genetic Engineering (KIBGE), University of Karachi., Karachi, Sindh, Pakistan

Abstract: Breast carcinoma is the most frequent cancer in women around the world. The risk factors which play a crucial role in development of breast carcinoma like gender, lifestyle, age, reproductive health, family history and hormonal levels. Studies have shown the regulatory mechanisms of cell cycle checkpoint gene(s) in others cancers. The aim of the study is to analyze the association of single nucleotide polymorphism (SNP) in the cell cycle regulatory gene (CHEK2) for breast cancer progression. A total of 250 female breast cancer patients and 200 healthy individuals as controls were included. Blood samples were collected with informed consent. The extraction of DNA was done by standard phenolchloroform method followed by tetra-primers Amplified Refractory Mutation System (t-ARMS) PCR for the genotyping of rs200928781 of 
CHEK2 gene. The Statistical Analyses was done using SPSS software 22. The Odds and risk ratio was calculated through an online calculator tool. Genotypic analysis reveals that the homozygous mutant allele $(G)$ was significantly higher in BC patients. Pearson Chi-Square value 219 with pvalue $<0.0001$ showed that SNP (rs200928781) is significantly associated with breast carcinoma. Odds ratio value 21.02 indicates that this SNP might be playing a role in cancer progression. These results of the current study might be helpful to improve the genetic diagnosis of breast cancer. The major causes of breast cancer is genetic. In this study, mutation has been investigated one of the six breast cancer specific genes called CHEK2 which codes for a protein known as checkpoint kinase 2, involved in regulating $\mathrm{G} 1$ and $\mathrm{G} 2$ checkpoints in the cell cycle. Objectives: To analyze the association of single nucleotide polymorphism (SNP) in the cell cycle regulatory gene (CHEK2) for breast cancer progression. References: Prognostic Significance of CHEK2 Mutation in Progression of Breast Cancer. Ansari N, Shahrabi S, Khosravi A, Shirzad R, Rezaeean H.Lab Med. 2019 Jul 16;50(3):e36-e41. doi: 10.1093/ labmed/lmz009.Germline CHEK2 Gene Mutations in Hereditary Breast Cancer Predisposition - Mutation Types and their Biological and Clinical Relevance. Kleiblová $\mathrm{P}$, Stolařová L, Kř́žová K, Lhota F, Hojný J, Zemánková $\mathrm{P}$, Havránek $\mathrm{O}$, Vočka M, Černá M, Lhotová K, Borecká M, Janatová M, Soukupová J, Ševčík J, Zimovjanová M, Kotlas J, Panczak A, Veselá K, Červenková J, Schneiderová M, Burócziová M, Burdová K, Stránecký V, Foretová L, Macháčková E, Tavandzis S, Kmoch S, Macůrek L, Kleibl Z.Klin Onkol. 2019 Summer;32(Supplementum2):36-50. doi: 10.14735/amko2019S36.

P3: Genetic Variation(s) in Hypoxia-Associated Gene Carbonic Anhydrase 9 (CA9) in Squamous Cell Carcinoma of Lungs and Esophagus

Saima Saleem, Ramsha Khan

The Karachi Institute of Biotechnology and Genetic Engineering (KIBGE), University of Karachi., Karachi, Sindh, Pakistan

\begin{abstract}
Squamous cell carcinoma of the esophagus and lungs pose a serious threat to health. High energy and metabolic demands within the rapidly proliferating tumor cells lead to hypoxia, causes a homeostatic imbalance in the tumor microenvironment. Expression of key hypoxiaassociated genes like CA9 has a prominent role in tumor survival. Approval for the use of human subjects from ethical review committees and written informed consent was taken from all subjects. The genomic DNA of 200 patients and control subjects was extracted through the standard phenol-chloroform method. To assess the targeted variant, $\mathrm{t}$ ARMS PCR was used. Statistical analyses were done using SPSS 22 software. The odds ratio and risk ratios were calculated by using online tools. This study found the association of missense polymorphism rs2071676 (+201 G>A), which changes valine to methionine and lies in the signal peptide domain of CAIX. Pearson's chi-square and odds ratio value of for LSCC and ESCC, showed a statistically significant association, at $\alpha=0.05$ and $p<0.0001$. It could be inferred that rs2071676 may be involved in disease progression. Furthermore, the effect of smoking and chewing addiction was observed, smoking habit gave no significant association, while chewing addicts were found to be significantly associated with ESCC with $\mathrm{p}<0.001$, influencing disease progression in combination with the polymorphism. Objectives: To find the expression of key $\mathrm{pH}$ regulatory proteins in tumor-associated hypoxia might be a prominent causative factor for the survival and invasiveness of different types of malignant tumors. To detect the CA9 gene polymorphism encodes for a membrane-bound enzymatic protein called Carbonic Anhydrase 9 that is released in high concentration by the cancer cells in response to hypoxia-mediated metabolic shift. To find the CA9 protein has been reported through various studies, to play pivotal roles in maintenance of the intracellular $\mathrm{pH}$ and might help in metastasis of tumor cells by facilitating angiogenesis. To analyze genetic variation(s) in CA9 gene.
\end{abstract} References: MN/CA9 as a novel molecular marker for the detection of cancer. Li G, Feng G, Achour Y, Genin C, Tostain J. Expert Opin Med Diagn. 2007 Sep;1(1):91-7. doi: 10.1517/17530059.1.1.91.PMID: 23489271.

017Expression of vascular endothelial growth factor, hypoxia inducible factor 1alpha, and carbonic anhydrase IX in human tumours. Jubb AM, Pham TQ, Hanby AM, Frantz GD, Peale FV, Wu TD, Koeppen HW, Hillan KJ.J Clin Pathol. 2004 May;57(5):504-12. doi: 10.1136/ jcp.2003.012963.

\section{P4: Clinical Aspects Provide Essential Components of a Translational Approach to Undergraduate Cancer Education}

Bret Hassel $^{1}$, Rena Lapidus ${ }^{1}$, Lauren Schnaper ${ }^{2}$, Kevin Cullen ${ }^{1}$, Heather Ezelle $^{1}$

${ }^{1}$ University of Maryland Greenebaum Cancer Center, Baltimore, MD, USA, ${ }^{2}$ Greater Baltimore Medical Center, Baltimore, MD, USA

Abstract: The Nathan Schnaper Intern Program in Translational Cancer Research has grown from a laboratory-focused undergraduate experience to include an intensive curriculum that integrates diverse aspects of translational research. Comprised largely of aspiring physician-scientists, intern cohorts expressed interest in incorporating clinical experiences into the curriculum that would illustrate the therapeutic applications of their research and provide exposure to the human face of cancer. Developing these clinical components is the goal of this project. In response to intern input, the clinical mentor pool grew by over $500 \%$ and the number of oncology specialties for interns to shadow increased 8-fold in the past decade. Two translational research modules that emphasize the link between laboratory research and clinical applications were developed: i) interns dissect pioneering work to develop aromatase inhibitors for breast cancer therapy; ii) Oncology Fellows serve as preceptors to prepare interns for patient cases discussed in Leukemia Boards. Outcomes enhanced cancer education in the internship: i) the clinical mentor pool and oncology specialties increased providing expanded shadowing opportunities; ii) a module in which interns dissect Dr. Angela Brodie's work to develop breast cancer therapeutics was piloted; iii) a module in which Oncology Fellows serve as preceptors to prepare interns for participation in Leukemia Boards was implemented. Intern evaluations revealed that clinical components provide valuable insights into diverse professional paths and enhance their understanding of cancer biology and treatment. These new components have been incorporated as regular sustainable features of the internship curriculum. Clinical modules and experience developed through this project inspire interns from diverse backgrounds to pursue careers in cancer-focused research and oncology and inform their decisions on this path to fulfill major goals of the program. Objectives: The participant shall be able to provide examples of how clinical experiences function in cancer education to foster interns' perspective of translational research. The participant shall be able to articulate the benefits of clinical experiences to undergraduate interns' educational and career path decisions. The participant shall be able to discuss ways in which patient exposure is critical for interns to appreciate the impact of social determinants on clinical outcomes. References: Howell, LP, Wahl, S, Ryan, J, Gandour-Edwards, R and Green, R 2019. Educational and Career Development Outcomes Among Undergraduate Summer Research Interns: A Pipeline for Pathology, Laboratory Medicine, and Biomedical Science. Acad. Pathol. Dec 13;6:2374289519893105. doi: 10.1177/2374289519893105. Kolber BJ, Jancic JM, Pollock JA, Tidgewell KJ. 2016 Summer research undergraduate research: a new pipeline for pain clinical practice and research. BMC Med Educ. 16:135. doi:10.1186/s12909-016-0648-7.

P5: Meeting the information and support needs of people with advanced cancer using a co-design methodology

Tracy Torchetti, Clodagh McCarthy

Canadian Cancer Society, Toronto, ON, Canada 
Abstract: In 2019 CCS developed an evidence-based roadmap to identify the information and support needs of underserved populations including patients diagnosed with advanced disease. The roadmap set the groundwork for conducting an in-depth study of audience needs that would result in a co-designed publication about advanced cancer. The research on the needs of people with advanced cancer used a variety of methods, including literature review, expert interviews, ethnographic interviews and a heuristic review of current CCS resources. These findings were leveraged for a digital workshop with people with advanced cancer, their caregivers and experts. The workshops validated the initial findings and tested copywriting and visual stimulus to inform the design and content direction of the publication. The research outcomes included writing and design principles to inform the final product, a table of contents and prototypes for structure and content. The research also identified gaps and opportunities for CCS to address both within the publication and through support programs to better engage with this population. CCS prioritized clients living with advanced disease, undertaking a review of its advanced cancer publication and support services to better meet their needs. The generative research for the publication will be an input into other resources and a second phase of research that specifically focuses on co-designing support services. Objectives: Participants will learn about a co-design approach to publication development, the outcomes of generative research and how CCS plans to implement the findings for both product development and support services. References: Unmet care needs in people living with advanced cancer: a systematic review. Moghaddam N, Coxon H, Nabarro S, Hardy B, and Cox K. Supportive Care in Cancer, ISSN 0941-4355, 8/2016, Volume 24, Issue 8, pp. $3609-$ 3622. Triadic treatment decision-making in advanced cancer: a pilot study of the roles and perceptions of patients, caregivers, and oncologists. LeBlanc TW, Bloom N, Wolf SP, Lowman SG, Pollak K, Steinhauser KE, Ariely D, and Tulsky JA. Supportive Care in Cancer, ISSN 09414355, 4/2018, Volume 26, Issue 4, pp. 1197 - 1205.

P6: Sociodemographic and Contextual Factors Associated with Trust in Physicians among Hispanic Caregivers of Children with Newly-Diagnosed Cancer

Joseph Morales ${ }^{1}$, Carolyn Rocha ${ }^{1}$, Shilpa Nataraj ${ }^{1}$, Bianca Perdomo ${ }^{1}$, Nassim Durali ${ }^{1}$, Nikhil Kumar ${ }^{1}$, France Nguyen-Grozavu ${ }^{1}$, Georgia Robins Sadler $^{1}$, Paula Aristizabal ${ }^{2}$

${ }^{1}$ UCSD Moores Cancer Center, La Jolla, CA, USA, ${ }^{2}$ Peckham Center for Cancer and Blood Disorders, Rady Children's Hospital San Diego, San Diego, CA, USA

Abstract: Caregivers' trust in physicians can help achieve optimal outcomes in pediatric cancer. Research shows that African-Americans report lower trust in physicians. Data on trust in physicians among other minority caregivers of children with cancer is lacking. This study explored factors associated with trust among Hispanic caregivers of children with cancer. The Pediatric Trust in Physician Scale (Pedi-TIPS) was completed prospectively in a convenience sample of 140 caregivers of children with newly-diagnosed cancer within six months of the cancer diagnosis at Rady Children's Hospital-San Diego. To assess contextual and sociodemographic factors associated with trust in physicians, health literacy was assessed with the Newest Vital Sign, acculturation with the Short Acculturation Scale for Hispanics, and socio-demographic characteristics with a socio-demographic questionnaire (age, income, number of children in household, insurance, ethnicity, preferred spoken/written language for medical information). Associations were assessed with linear regression models. Forty-nine percent of the participants $(n=69)$ were Hispanic, 51\% ( $\mathrm{n}=71)$ were non-Hispanic Whites (NHW) and $67 \%$ were the children's mother. The mean difference of the Pedi-TIPS scores between Hispanics and NHW was $0.081(\mathrm{p}=0.934)$. Disparate levels of trust in physicians were only correlated with caregivers' number of children: caregivers with one child scoring higher in trust than those who had two or more children $(p=0.006)$ with a mean score of 46.6 . Results suggest trust levels in Hispanic caregivers of children with cancer may not play the same role as in African-Americans. Trust in physicians was comparable for Hispanic and NHW caregivers but lower among caregivers with larger. Future research is needed to assess trust in a larger, diverse sample and understand the influence of family size when establishing trust with a physician and variations among different minority racial/ethnic groups. Objectives: The participants shall be able to understand that there are not statistically different data indicating that race, income level, language spoken, whether or not the participants lived in a rural area, insurance type, parents age, child age, education level, employment status, religion, marital status did not play a factor in the levels of trust they had with their child's physician. The participant shall be able to notice that larger families have a tendency to be more distrustful with the child's physician. References: Moseley, K. L., Clark, S. J., Gebremariam, A., Sternthal, M. J., \& Kemper, A. R. (2006). Parents' Trust in Their Child's Physician: Using an Adapted Trust in Physician Scale. Ambulatory Pediatrics, 6(1), 58-61. doi: 10.1016/ j.ambp.2005.08.001. Corbie-Smith, G., Thomas, S. B., \& St. George, D. M. (2002). Distrust, Race, and Research. Archives of Internal Medicine, 162. doi: 10.1001/archinte.162.21.2458

\section{P7: Systematic Review of Colorectal Cancer Screening using Annual Stool Blood Testing focused on African Americans}

Siddhartha Roy ${ }^{1}$, Sabrina Dickey ${ }^{2}$, Hsiao-Lan Wang ${ }^{3}$, Alexandria Washington ${ }^{4}$, Randy Polo ${ }^{5}$, Tierah Edwards ${ }^{4}$, Clement Gwede ${ }^{7}$, John Luque $^{4}$

${ }^{1}$ Penn State College of Medicine, Hummelstown, PA, USA, ${ }^{2}$ College of Nursing, Florida State University, Tallahassee, FL, USA, ${ }^{3}$ College of Nursing, University of South Florida, Tampa, FL, USA, ${ }^{4}$ Florida A\&M University, Tallahassee, FL, USA, ${ }^{5}$ University of South Florida Health Libraries, Tampa, FL, USA, ${ }^{6}$ Moffitt Cancer Center, Tampa, FL, USA

Abstract: African Americans experience colorectal cancer (CRC) related disparities compared to other racial groups in the United States. African Americans are frequently diagnosed with $\mathrm{CRC}$ at a later stage, screening is underutilized, and mortality rates are highest in this group. This systematic review focused on intervention studies using stool blood CRC screening among African Americans in primary care and community settings. Given wide accessibility, low cost, and ease of dissemination of stool-based CRC screening tests, this review examined the effectiveness of interventions to improve participation rates. This systematic review included intervention studies published between January 1, 2000 and March 16, 2019. After reviewing an initial search of 650 studies, 11 studies were included in this review. The included studies were studies conducted in community and clinical settings, using both inreach and outreach strategies to increase CRC screening. For each study, an unadjusted odds ratio (OR) for the CRC screening intervention compared to the control arm was calculated based on the raw data in each study to determine effectiveness. The eleven studies together recruited a total of 3,334 participants. The five studies using two-arm experimental designs ranged in effectiveness with ORs ranging from 1.1 to 13.0 using interventions such as mailed reminders, patient navigation, and tailored educational materials. Effective strategies to increase stool blood testing included mailed blood stool tests augmented by patient navigation, tailored educational materials, and follow-up calls or mailings to increase trust in the patient-provider relationship. More studies are needed on stool blood testing interventions to determine effectiveness in this population. Objectives: The participant shall be able to weigh the effectiveness of different types of inreach and outreach strategies that may be effective to increase colorectal cancer screening in African American communities. References: Carethers, J. M. 2018. Clinical and Genetic Factors to Inform Reducing Colorectal Cancer Disparitites in African Americans. Front Oncol 8:531. doi:10.3389/fonc.2018.00531. Coughlin, S. S., D. S. Blumenthal, S. J. Seay, and S. A. Smith. 2016. Toward the Elimination of Colorectal Cancer Disparities Among African Americans. J Racial Ethn 
Health Disparities 3 (4):555-564. doi:10.1007/s40615-015-0174-z. Williams, R. M., T. Wilkerson, and C. L. Holt. 2018. The role of perceived benefits and barriers in colorectal cancer screening in intervention trials among African Americans. Health Educ Res 33 (3):205-217. doi:10.1093/her/cyy013.

\section{P9: The Relationship between Masculinity Traits and Men's Health Behaviors}

Bidemi Adedokun, Isela Garcia, Anne Hubbell, Rebecca Palacios New Mexico State University, Las Cruces, NM, USA

\begin{abstract}
Empirical studies suggest that men are more likely than women to engage in risky health behaviors which increases their risk of injury, disease, and death. Researchers suggest that socialization of male gender roles contributes to higher frequencies of risk behaviors and poor health outcomes. A literature review of studies examining the relationship of masculinity traits to men's health behaviors was conducted using PsycINFO, PubMed, PsycArticles, and PsycTest. The final review retained peer-reviewed articles that examined the influence of masculinity traits on health behaviors in men and which were published between 2000 and 2019. Thirty-one articles were identified, listing 6 different inventories of masculinity traits. Fifteen unique masculinity constructs were identified across the 6 inventories and assessed. These included Winning, Risk Taking, Dominance, Primacy of Work, Self-Reliance, Emotional Control, Sexual Importance, Hypermasculinity, Optimistic Capacity, Strength, Responsibilities/Family, Reputation, Action Approach, Conservative Masculinity, and Bias against sexual minorities. Three masculinity constructs were associated with high engagement, two constructs with low engagement, and two constructs with both high and low engagement in risky behaviors. Five constructs were associated with low engagement, three constructs with high engagement, and five with both high and low engagement in healthy lifestyles. Although the literature typically portrays masculine traits as having negative influence on men's health behavior, these findings suggest that masculine traits can predict both positive and negative health behaviors in men. These findings can help direct the development of cancer prevention interventions for men. Objectives: The participant shall be able to identify the masculinity traits that predict high-risk behaviors. The participant shall be able to identify the masculinity traits that predict poor lifestyle. The participant shall be able to identify the masculinity traits that predict low engagement in risk behaviors. The participant shall be able to identify the masculinity traits that predict healthy lifestyle. References: Chambers, S. K., Hyde, M. K., Oliffe, J. L., Zajdlewicz, L., Lowe, A., Wootten, A. C., \& Dunn, J. (2016). Measuring masculinity in the context of chronic disease. Psychology of Men \& Masculinity, 17(3), 228. https://doi.org/10.1037/ men0000018. Ramon, A. E., Guthrie, L., \& Rochester, N. K. (2019). Role of masculinity in relationships between mindfulness, selfcompassion, and well-being in military veterans. Psychology of Men \& Masculinities. https://doi.org/10.1037/men0000240.
\end{abstract}

\section{P10: Childhood Cancer Survivorship among Minority Populations}

Juan Andrew Leal, Anthony Cirilo, Gavrila Ang, France NguyenGrozavu, Suzi Hong, Georgia Robins Sadler UCSD Moores Cancer Center, La Jolla, CA, USA

Abstract: Non-Hispanic white children aged zero to 19 have a higher cancer survival rate than their minority counterparts. This narrative literature review explored social factors reported to positively and negative impact childhood cancer survivorship and long-term health outcomes impacting adulthood. Evidence-based methods were explored to identify strategies to reduce those disparities. A search of scientific literature, written in English or Spanish, related to childhood and adolescent cancer and its impact on health later in life was conducted. The following databases were searched for articles published since 2011: PubMed,
PsycINFO, CINAL, BIOSIS, Embase, Web of Science, and ERIC. Major search terms included: cancer, childhood, children, teen, adolescent, survivorship, disparity, African, Hispanic, Asian, Native American, low income, minority, and the names of common childhood cancers. Reference lists of eligible articles were reviewed to identify additional published articles with no date restriction. Only articles with full-text accessibility were eligible for inclusion. This review reconfirmed the lower childhood cancer survival rate among minority children compared to non-Hispanic white children. Low socio-economic status, increased distance from National Cancer Institute-designated cancer center and limited access to information were negatively correlated with cancer mortality rates among minority children. Childhood cancer survivors also experience consequential health issues later in life. This review showed that adult minority survivors suffered from poorer health outcomes in adulthood than their non-Hispanic white counterparts. However, a greater appreciation for life was observed in minority survivors, though a lack of follow up care and adherence to healthy lifestyles persists throughout adulthood. Targeted interventions and increased surveillance must be implemented to increase cancer knowledge and promote healthy lifestyles in minority childhood cancer survivors to help reduce the identified disparities. Systematic procedures supporting rural populations' access to healthcare and streamlining the dissemination of health information is crucial in increasing cancer survivorship. Objectives: The participant shall be able to identify at least three barriers impacting the survivorship of childhood cancer among minority populations and provide evidencebased methods to mitigate those barriers. References: Delavar, A., Barnes, J. M., Wang, X., \& Johnson, K. J. (2020). Associations Between Race/Ethnicity and US Childhood and Adolescent Cancer Survival by Treatment Amenability. JAMA Pediatrics, 174(5), 428. doi:10.1001/jamapediatrics.2019.6074. Salchow, J., Mann, J., Koch, B., Grundherr, J. V., Jensen, W., Elmers, S.,... Stein, A. (2020). Comprehensive assessments and related interventions to enhance the long-term outcomes of child, adolescent and young adult cancer survivors - presentation of the CARE for CAYA-Program study protocol and associated literature review. BMC Cancer, 20(1). doi:10.1186/s12885-0196492-5.

P11: Strategies to Improve Prostate Cancer Mortality Rates among Native Americans and Alaska Natives

Hao Pham, Christina Jamieson, Georgia Robins Sadler, France NguyenGrozavu

UCSD Moores Cancer Center, La Jolla, CA, USA

Abstract: The ratio between the prostate cancer incident rate and death rate was higher for Native Americans and Alaska Natives compared to other races. Multiple causes have been reported for this survival disparity. The next step is to identify the various intervention strategies applicable to these communities. A narrative review of scientific literature was undertaken to identify and evaluate strategies that have been reported for improving prostate cancer survival rates among NA/AN. Articles published in PubMed, CINAHL, SAGE journals, and Google Scholar between 2010 and 2020 were included. Websites were reviewed from such organizations as, the National Cancer Institution, American Cancer Society, and Centers for Disease Control and Prevention. Search terms included: prostate cancer, incidence, mortality, survival, risk factors, lifestyle, culture, genetic, genome, Alaska Native, American Indian, Native American, access to care, trust, mental, early detection, and treatment. Reference lists of eligible articles were also reviewed This review identified three promising new strategies with potential for improving prostate cancer survival rates among NA/AN. First, the Prostate Urine Risk Test (PUR), a preliminary self-administered prostate cancer detection test that may overcome modesty, cost, discomfort, and geographic barriers to office screening. Second is the Patient Reported Information Multidimensional Exploration framework that helps doctors evaluate patients' emotional fluctuations through online screening and referral to 
online cancer support groups to help patients become better informed and cope better with intense negative emotions. Third, partnering NA/AN prostate cancer survivors with newly diagnosed men can educate and support newly diagnosed cancer patients. These three strategies offer a chance for reducing prostate cancer deaths among NA/AN. It is imperative that researchers specifically evaluate their benefits for those communities. Cultural humility participant recruitment strategies and trusted community leaders' endorsement will be essential to evaluate these strategies. Objectives: The participant shall be able to learn about a new home collection protocol for prostate cancer that may overcome cost, discomfort, and geographic barriers for Native Americans and Alaska Natives (NA/AN). In addition, the participant shall be able to learn new mental support techniques that a doctor can use to indicate patience's negative emotions without a physical examination. Lastly, the participant shall be able to see the way to educate and support newly diagnosed cancer patients from NA/AN community. References: Webb, M., Manley, K., Olivan, M., Guldvik, I., Palczynska, M., Hurst, R., ... Clark, J. (2020). Methodology for the at-home collection of urine samples for prostate cancer detection. BioTechniques, 68(2), 65-71. doi: 10.2144/btn-20190092. Bastian, T. D., \& Burhansstipanov, L. (2020). Sharing Wisdom, Sharing Hope: Strategies Used by Native American Cancer Survivors to Restore Quality of Life. JCO global oncology, 6, 161-166. https:// doi.org/10.1200/JGO.19.00215.

\section{P12: Exploring the Age-Related Role of NUP210 in ER+ Breast Cancer}

Dalia Koujah ${ }^{1}$, Georgia Robins Sadler ${ }^{1}$, Maximiliano D'Angelo ${ }^{2}$, Marcela Raices ${ }^{2}$

${ }^{1}$ UCSD Moores Cancer Center, La Jolla, CA, USA, ${ }^{2}$ Sanford Burnham Prebys Medical Discovery Institute, La Jolla, CA, USA

Abstract: Disparities in breast cancer morbidity and mortality rates exhibit a differential increase with age. NUP210 is a nucleoporin that has been linked to cancer metastasis, particularly in Estrogen Receptor (ER+) breast cancer. This study explores how NUP210 expression varies with age and correlates with poor prognosis in patients $65+$. A narrative review of scientific literature explored interactions between NUP210 and breast cancer metastasis. Next, data on patient information and outcomes were compiled from The Cancer Genome Atlas (TCGA) and cBioPortal. Patient data from cBioPortal were categorized into age groups, and samples were further narrowed down to select for ER+ breast cancer, where NUP210 has been found to regulate metastasis $(n=1825)$. NUP210 expression was quantified using normalized RSEM (RNA-seq by Expectation-Maximization) values, which represent protein expression for each patient. Survival curves were generated for different age groups, and a p-value below 0.05 was used to determine statistical significance. The literature identifies NUP210 as a metastasis susceptibility gene. Data from TCGA confirms that NUP210 is highly expressed in breast cancer, relative to cancer in other tissues. Expression of NUP210 mRNA showed a statistically significant increase in patients $65+$ compared to patients below 65 . This overexpression correlates with such factors as higher tumor cellularity and staging in older patients. The literature indicates that older patients are undertreated and underrepresented in clinical trials, due to age restrictions and the presence of comorbidities. This study suggests the need for age-specific breast cancer clinical trials in order to improve outcomes for patients $65+$. Breast cancer research can be enhanced through interdisciplinary collaborations of clinicians and research scientists. Collaborations interested in studying population subgroups will encourage the accrual of well-diversified samples of participants. This study demonstrated the value of studying a subsample of older women with the potential to inform personalized treatment plans. Objectives: The participant shall be able to identify a genetic risk factor contributing to high breast cancer morbidity and mortality rates in elderly patients. The participant shall be able to identify barriers to improved health outcomes for elderly breast cancer patients. References: Amin,
R. et al. Targeting nuclear pore protein, NUP210, reduces metastasis through heterochromatin-mediated silencing of mechanosensitive genes. (2020). Johansson, A. L. et al. Breast cancer-specific survival by clinical subtype after 7 years follow-up of young and elderly women in a nationwide cohort. International Journal of Cancer 144, 1251-1261 (2018).

\section{P13: Understanding how early adolescents think about the HPV vac- cine}

Katherine Ayers ${ }^{1}$, Heather Brandt ${ }^{2}$, Robyn Pennella ${ }^{1}$

${ }^{1}$ St. Jude Children's Research Hospital, Memphis, TN, USA, ${ }^{2}$ University of South Carolina, Columbia, SC, USA

Abstract: HPV vaccination uptake in the United States (U.S.) has lagged, and uptake in Tennessee is among the lowest in the U.S. This exploratory qualitative study examined how early adolescents think about the HPV vaccine to identify patterns and misconceptions that can be used to better tailor future educational efforts. During a 5-day summer cancer education program in Tennessee, rising 6th-grade students $(n=168)$ completed written, open-ended questions regarding their thinking around the HPV vaccine as part of a larger workbook. Specifically, students were asked: 1) What excites you about the HPV vaccine? What's the upside? 2) What worries you about the HPV vaccine? What's the downside? 3) What do you still need to know about the HPV vaccine? 4) What is your stance or opinion on the HPV vaccine? Responses were transcribed and summarized using qualitative content analysis. About two-thirds of students expressed a positive opinion of the HPV vaccine while only a few expressed a negative opinion. Prevention was the most commonly cited upside to getting the vaccine while worries about administration of the shot, efficacy, and side effects were seen as potential downsides. Some students also expressed worries about low vaccination rates in the community and their risk of potential infection and/or developing HPVrelated cancers. Students wanted more knowledge on a variety of topics related to the vaccine, including vaccine composition, the body's response to the vaccine, and immunization duration. Student attitudes were mostly positive towards the HPV vaccine. Several themes emerged highlighting specific questions early adolescents have about the vaccine that can be used to enhance HPV educational efforts. In addition, the potential for early adolescents to serve as community advocates for the vaccine should be considered for future Objectives: The participants shall be able to identify strategies for developing HPV-related health education for young adolescents. References: Thompson, E.L., Rosen, B.L. \& Maness, S.B. Social Determinants of Health and Human Papillomavirus Vaccination Among Young Adults, National Health Interview Survey 2016. J Community Health 44, 149-158 (2019). https://doi.org/10.1007/s10900-018-0565-2. Emilie Karafillakis, Clarissa Simas, Caitlin Jarrett, Pierre Verger, Patrick Peretti-Watel, Fadia Dib, Stefania De Angelis, Judit Takacs, Karam Adel Ali, Lucia Pastore Celentano \& Heidi Larson (2019) HPV vaccination in a context of public mistrust and uncertainty: a systematic literature review of determinants of HPV vaccine hesitancy in Europe, Human Vaccines \& Immunotherapeutics, 15:7-8, 1615-1627, DOI: 10.1080/21645515. 2018.1564436

\section{P14: Suggestions for Interventions to Promote Cervical Cancer Screening in Surat, India}

Dina Koes ${ }^{1}$, Apurva Barve ${ }^{2}$, Vipul Chaudhari ${ }^{3}$, Janna Gordon ${ }^{4}$, Alap Mehta $^{5}$, Jayendrakumar Kosambiya ${ }^{2}$, Ambuj Kumar ${ }^{6}$, Kristen Wells ${ }^{1}$, Sukesha Gambit ${ }^{3}$

${ }^{1}$ San Diego State University, San Diego, CA, USA, ${ }^{2}$ University of California, Los Angeles, CA, USA, ${ }^{3}$ Government Medical College, Surat, Surat, Gujarat State, India, ${ }^{4} S D S U / U C$ San Diego Joint Doctoral Program in Clinical Psychology, San Diego, CA, USA, ${ }^{5}$ New Civil Hospital, Surat, Surat, Gujarat State, India, ${ }^{6}$ University of South Florida, Tampa, FL, USA 
Abstract: Cervical cancer is the second leading cause of death for women in India. Women living with HIV are recommended to have more frequent cervical cancer screening, yet, few interventions have been developed. This qualitative study assessed suggestions for screening intervention approaches among women living with HIV, and among healthcare providers. The Social-Ecological Model (SEM) and the process of Intervention Mapping guided the study. We conducted semi-structured individual in-depth interviews with 25 women living with HIV (mean age: 37.2 years; $48 \%$ completed secondary school education) and 15 health care providers (mean age: 36.1 years; $66.6 \%$ female). All participants were affiliated with the New Civil Hospital, a public hospital in Surat, India. Interviews were audio-recorded, transcribed verbatim, deidentified, translated, if necessary, into English by multilingual study staff, and analyzed using content analysis. Patients suggested an intervention should provide detailed information about cervical cancer, what will happen during cervical cancer screening, and why it is important. Most study participants stressed the importance of developing an intervention that makes it easier to obtain cervical cancer screening and care, such as through improved communication. Healthcare providers and patients indicated that female physicians and counselors should deliver the intervention. Participants suggested both community and clinical settings were appropriate for providing cervical cancer screening interventions. This study is one of the first to obtain the preferences of both Indian women living with HIV and their health care providers for developing an intervention facilitating recommended $\mathrm{CC}$ screening. These data can be used to develop a CC screening intervention in either a clinic or community setting. Objectives: The participants will be able to identify the preferences of Indian women living with HIV and health care providers in developing an intervention to facilitate recommended cervical cancer screening. References: Gordon, J., Barve, A., Chaudhari, V., Kosambiya, J., Kumar, A., Gamit, S., \& Wells, K. (2019). "HIV is not an easily acceptable disease": The role of HIV-related stigma in obtaining cervical cancer screening in India. Women \& Health, 59(7), 801-814. Kung, T., Gordon, H., Abdullahi, J., Barve, R., Chaudhari, A., Kosambiya, A.,... Wells, K. (2019). "My husband says this: If you are alive, you can be someone...": Facilitators and barriers to cervical cancer screening among women living with HIV in India. Cancer Causes \& Control, 30(4), 365-374.

P15: Hepatitis B testing among Vietnamese immigrants in Atlanta: The role of healthcare- and acculturation-related predictors

Milkie Vu, Victoria Huynh

Emory University, Atlanta, GA, USA

Abstract: Hepatitis B infection is a major risk factor for liver cancer. The prevalence of Hepatitis B infection among Vietnamese-Americans is between $8.8 \%-13.8 \%$ (compared to $0.36 \%$ in general U.S. populations) [14]. Increased testing could reduce this disparity. This study sought to examine potential predictors of Hepatitis B among VietnameseAmericans. We administered surveys to 100 Vietnamese adults recruited through community health fairs and programs in Atlanta in 2017. Healthcare-related predictors included insurance and health checkup within the past 2 years. Acculturation-related predictors included Vietnamese enculturation scores and American acculturation scores (measured through the Vancouver Acculturation Index), the percentage of lifetime in the U.S., and Vietnamese and English fluency. Additional sociodemographic variables included age, sex, highest education attained, and annual household income. Multiple imputation was used for missing data. A multivariable logistic regression was conducted to examine the association between predictors and the outcome of Hepatitis B testing. Of 100 participants, $61.6 \%$ were female and $44.3 \%$ reported an annual household income of $\$ 50,000$ or above. The mean age was 37.5 . The average percentage of lifetime in the U.S. was $56.8 \%$. Of the sample, $70.0 \%$ reported having ever tested for Hepatitis B. In a multiple logistic regression, having ever tested for Hepatitis $\mathrm{B}$ was associated with having health insurance $(\mathrm{aOR}=2.61,95 \% \mathrm{CI}=[1.05-6.47], \mathrm{p}=.04)$. No other acculturation-related or sociodemographic predictors were associated with Hepatitis B testing. Compared to Vietnamese-Americans with no insurance, those with insurance were more likely to have ever been tested for Hepatitis B. Effective strategies to increase Hepatitis B testing and reducing liver cancer burden among Vietnamese-Americans may require addressing access to health insurance. Objectives: Describe the gaps in existing literature concerning testing for Hepatitis B among VietnameseAmericans. Identify predictors of Hepatitis B testing among a high-risk population.

Describe the significance of access to health insurance for Hepatitis B testing among a high-risk population. References: 1 . https://doi.org/10. 1093/cid/ciz001. 2. https://doi.org/10.1007/s10903-013-9975-5. 3. https://doi.org/10.1007/s10903-014-0015-x. 4. https://doi.org/10.1136/ bmjopen-2019-029616.

\section{P16: Benefits for using Kazakh population for detecting Esophageal Squamous Cell Carcinoma}

Esau Estrada, Georgia Robins Sadler, France Nguyen-Grozavu, Miguel Lopez-Ramirez

UCSD Moores Cancer Center, La Jolla, CA, USA

Abstract: Esophageal squamous cell carcinoma (ESCC) occurs more commonly outside the United States. The Kazakhs, a small, nomadic Turkish ethnic group, have disproportionately high rates of ESCC. When a group has a disproportionate burden of illness, it is easier to study within-group differences in genetic profiles, living habits, and environmental influences. Cancer education programs translate scientific discoveries into recommended community actions and promote research participation. A basic science literature review demonstrates why diverse study participants are critical. Databases used for this literature review about ESCC among Kazakhs include PubMed, PsychINFO, EBSCO, ScienceDirect, and CINAHL. Full-text accessible articles written in English or Spanish between 2010 and 2020 were included. The search used such keyword variations as Kazakhs, Esophageal Squamous Cell Carcinoma, genetics, risk factors, environment, and lifestyle. It also focused on the investigation of specific mRNA (protein pathways) that scientist have shown contribute to tumor inhibition and proliferation among the Kazakhs population. Engaging Kazakhs in research enabled verification of hypothesized exacerbators of tumor etiology. Research demonstrated that ESCC tumors' aggressiveness increased with smoking, alcohol, and such cultural habits as consumption of a heavily-based red meat diet. In the presence of risk factors, aggressive vasculature formation increases through VEGF-C, a pathway where genes, such as SATB1, may play a role. These oncogenic gene insights were more readily discoverable by studying the highly-impacted, Kazakhs population. While ESCC initiation and treatment remain unclear, interventions based on these discoveries may help to slow tumor growth, while also demonstrating the importance of research with diverse populations. Within-group analysis of Kazakhs facilitated etiological understanding and verified participating signaling pathways of ESCC. While such investigations can advance ESCC's therapeutic management and suggest possible prevention strategies for investigation, this review also offers cancer educators an excellent example for explaining the importance of research participation by people of diverse characteristics. Objectives: The participant should be able to identify two current challenges of etiology for esophageal squamous cell carcinoma and consider the importance of preventive actions in health education. References: Li, J., Xie, Y., Wang, X., Jiang, C., Yuan, X., Zhang, A., ... Hu, J. (2019, December 3). Overexpression of VEGF-C and MMP-9 predicts poor prognosis in Kazakh patients with esophageal squamous cell carcinoma. Retrieved December 30, 2019, from https://www.ncbi.nlm.nih.gov/pmc/articles/ PMC6896941/. Song, G., Liu, K., Yang, X., Mu, B., Yang, J., Hu, X., ... Feng, G. (2017, January 27). SATB1 plays an oncogenic role in 
esophageal cancer by up-regulation of FN1 and PDGFRB. Retrieved December 19, 2019, from https://www.oncotarget.com/article/14849/ text/.

\section{P17: Diverse Biobank Contributions Unlock Potential Biological Disparities in Colorectal Cancer and Beyond}

Ethan Yi Zhu ${ }^{1}$, Maximiliano D'Angelo ${ }^{2}$, Georgia Robins Sadler ${ }^{1}$

${ }^{1}$ UCSD Moores Cancer Center, La Jolla, CA, USA, ${ }^{2}$ Sanford Burnham

Prebys Medical Discovery Institute, La Jolla, CA, USA

Abstract: The research utility in donating biological samples to a clinical data or specimen bank, known as biobanking, is often unclear to patients, clinicians, and the general public. This study shows the potential for biobank research in discovering cancer disparities and stresses the importance of building well-diversified databanks for effective research. Recent publications correlate increased POM121 expression with heightened colorectal cancer (CRC) aggressiveness. POM121 is one of $30+$ protein forming the nuclear pore complex, which controls movement between the nucleus and cytoplasm of a cell, and can regulate passage of proteins associated with increased cancer aggression. Expression of POM121 was evaluated in CRC using the racially-diverse TCGA patient sample database. POM121 expression data were retrieved and analyzed by race, patient survival, and T1-4 stages. Statistical testing was performed using unpaired t-test for two-group comparisons, and 1-way ANOVA with Tukey correction for multiple groups. African Americans demonstrate statistically significant increased POM121 expression compared to White patients among all CRC patients, and this difference increases when evaluating only deceased patients. However, analysis of data by race and stage showed no statistically significant increase for POM121 expression in African Americans for stages T2-4; T1 lacked sufficient African American sample size. Among deceased T4 patients, African Americans demonstrate statistically significant greater POM121 expression compared to Whites. Increased POM121 expression in African Americans can contribute to increased CRC aggression, and could be contributing to the overall disparity in African Americans' poorer CRC disease prognosis. These findings of potential biological differences in CRC by race can inform future individualized treatment while demonstrating the strength of, and disparities in basic biological biobank research due to sampling diversity. Patients and clinicians can facilitate the creation of well-diversified databanks, helping to achieve both wider generalizability and nuanced discoveries. Objectives: The participant shall be able to tangibly identify one example of how information obtained from clinical sample databanks can be analyzed to reveal potential molecular-level factors of race-associated differences in disease outcome. The participant shall also be able to recognize how promoting and building a well-diversified sample database can strengthen disease research in uncovering racial disparities in disease. The participant shall finally recognize the lack of patient background diversity in present-day clinical sample databases, preventing this potentially powerful research from taking place. References: Ansa, B., Coughlin, S., Alema-Mensah, E., and Smith, S. (2018) Evaluation of Colorectal Cancer Incidence Trends in the United States (2000-2014). Journal of Clinical Medicine, 7(2):22. doi:10.3390/jcm7020022. Tengqi Wang, Haibin Sun, Yinshengboer Bao, Riletu En, Yongjing Tian, Wei Zhao \& Lizhou Jia (2019) POM121 overexpression is related to a poor prognosis in colorectal cancer, Expert Review of Molecular Diagnostics, DOI: 10.1080/14737159.2020.1707670. Eggly, S., Barton, E., Winckles, A., Penner, L.A. and Albrecht, T.L. (2015), A disparity of words: racial differences in oncologist-patient communication about clinical trials. Health Expect, 18: 1316-1326. doi:10.1111/hex.12108.

P18: Cervical Cancer Screening, Practices, and Barriers among LGBTQ+ Community: A Literature Review

Mariah Romero, Georgia Robins Sadler, France Nguyen-Grozavu UCSD Moores Cancer Center, La Jolla, CA, USA
Abstract: The Centers for Disease Control rank cervical cancer as one of the most preventable cancers. LGBTQ+ people have disproportionately higher rates of being diagnosed with cervical cancer and at a later stage. This literature review explored remediable factors contributing to this excess disease burden. The scientific literature was examined to evaluate the barriers and policies that LGBTQ+ community members encounter that compromise the community's attainment of optimal cervical cancer control. The National Cancer Institute, Centers for Disease Control and Prevention, and the American Cancer Society websites were used along with such databases as PubMed, CINAHL, PsycInfo, and Google Scholar. Among the terms used for this search were: LGBTQ+, cervical cancer, screening, pap smear, women, health disparities, HPV vaccinations, accessibility, discriminatory policies, insurance, community outreach, fear of discrimination, and healthcare providers. Articles published since 2010 were eligible for inclusion. Limited access to quality healthcare is a major contributor to cervical cancer disparities within the LGBTQ+ community. Thirty percent of LGBTQ+ adults delay or don't seek healthcare services and lack a regular provider. They have a higher rate of participating in behaviors that increase the risk of cervical cancer, such as multiple partners and a high rate of sexual infections and diseases. There's a higher risk of poverty, lack of insurance coverage, and a lack of trust and understanding when disclosing sexual orientation or gender identity to a provider. Discriminatory feelings can occur when providers do not use correct pronouns. LGBTQ+ members face unique health challenges. Research shows that clinician-patient interactions strongly impact whether LGBTQ+ members seek routine healthcare. Research specific to sexual minorities is limited. Optional collection of sexual orientation in patients' charts and health registries could improve patient care and research. Community and provider education are also needed. Objectives: The participant shall be able to identify at least two barriers of routine cervical cancer screening for sexual minorities. One of the barriers that can be evaluated is that sexual minorities often times delay or do not seek healthcare services for fear of discrimination. Another barrier that can be evaluated is that sexual minorities have a higher risk of poverty and lack of insurance coverage. References: Boehmer, Ulrike. "Cancer Related Clinical Care of Sexual and Fender Minorities." CURE, 24 Feb. 2020, www.curetoday.com/advocacy/lgbtcancer/cancer-related-clinical-careof-sexual-and-fender-minorities-. Kates, Jennifer, et al. "Health and Access to Care and Coverage for Lesbian, Gay, Bisexual, and Transgender (LGBT) Individuals in the U.S." Kaiser Family Foundation, 3 May 2018, www.kff.org/disparities-policy/issue-brief/ health-and-access-to-care-and-coverage-for-lesbian-gay-bisexual-andtransgender-individuals-in-the-u-s/view/print/.

P19: Ductal Carcinoma in Situ (DCIS): An overlooked risk factor for invasive breast cancer(BC) in African American Women(AAW)

Nicole Lee, Thomas O'Keefe, Olivier Harismendy, Anne Wallace, Georgia Robins Sadler

UCSD Moores Cancer Center, La Jolla, CA, USA

Abstract: African American women (AAW) have disproportionately higher rates of breast cancer (BC) morbidity. Many factors are known to correlate to a higher risk of developing invasive $\mathrm{BC}$. This literature review examines one of those factors, ductal carcinoma in situ (DCIS) diagnosis, and its relationship to invasive $\mathrm{BC}$ in $\mathrm{AAW}$. An extensive literature review was conducted to explore whether there is any evidence that correlates race or ethnicity with higher BC mortality following an initial diagnosis of DCIS. The search was done on PubMed, PsychInfo, and Google Scholar using combinations and variations of the following terms: DCIS, African American, disparity, mortality, risk factors, barriers, distrust, and quality and promptness of treatment. Eligible articles ranged from November 2002 to February 2020. Reference lists from the eligible articles from the primary search were also examined. Those studies used databases gathered from DCIS patients in the United States, the United Kingdom, and Australia. DCIS, a noninvasive precancerous stage, was 
found to increase the risk of invasive BC. Many articles explained how AAW and white women typically had similar rates of invasive recurrence following a DCIS diagnosis. However, the AAW were typically diagnosed after the age of 65 with larger tumors than their white counterparts. This was attributed to the economic barrier of not being able to afford a mammogram until the age restrictions of Medicare could cover the costs. While DCIS alone does not contribute to higher mortality rates, it does increase the likelihood of developing a more aggressive illness. There are limited studies focused on whether DCIS contributes to AAW's higher risk of developing invasive $\mathrm{BC}$ and higher mortality rates. Further studies are needed to develop a better understanding of whether the differential mortality rates for AAW are associated with late stage detection of DCIS. Objectives: The participant will be able to identify one association between DCIS diagnosis and higher BC mortality rates in AAW. References: Bertrand KA, Bethea TN, Adams-Campbell LL, Rosenberg L, Palmer JR. Differential Patterns of Risk Factors for Early-Onset Breast Cancer by ER Status in African American Women. Cancer Epidemiol Biomarkers Prev. 2017 Feb;26(2):270-277. Narod SA, Iqbal J, Giannakeas V, Sopik V, Sun P. Breast Cancer Mortality After a Diagnosis of Ductal Carcinoma In Situ. JAMA Oncol. 2015;1(7):888-896.

P20: Are human trafficking victims also victims of increased cancer risk?

Jocelyn Quiroz, Georgia Robins Sadler

UCSD Moores Cancer Center, La Jolla, CA, USA

Abstract: Forty million people are modern-day slaves, trafficked for sexual, drug, and labor exploitation. The prevalence of mental, sexual, and physical health issues present among human trafficking victims make this cohort of grave concern. Yet, the health implications of human trafficking and their relation to cancer risk have received little attention. PubMed, CINAHL, PsycInfo, and Google Scholar were searched for relevant, accessible, full-text articles published since 2010 in English or Spanish on cancer statistics among victims of human trafficking, what cancer risk factors have been linked to victims of trafficking, and evidence-based studies undertaken to reduce victim's cancer risk. Relevant articles' reference lists were explored for additional relevant articles. Combinations of key search words used included: human, trafficking, sexual violence, child abuse, cancer, risk, mental health, learned helplessness, substance abuse, illegal abortions, drug mule, sex worker, domestic worker, HPV, HIV, AIDS, chronic inflammation, venereal disease, and oral, anal, and vaginal abuse. No studies linking cancer risk and human trafficking were found. One article investigated the likelihood of a relationship between human trafficking and cancer development through a literature search and identified the possibility of adulthood cancer resulting from 1) human trafficking or 2) behavior that developed in response to being trafficked. A multitude of health implications associated with human trafficking (often simultaneously experienced), including substance abuse, learned helplessness, immune system suppression, inadequate hygiene, illegal abortions, anal abuse, and oral abuse, could potentially increase cancer risk. Minorities and immigrants experience more extreme forms of exploitation, likely further raising cancer risk. The literature supports the likelihood of finding a correlation between elevated cancer risk and being a human trafficking victim with all of its associated cancer-inducing negative consequences. Direct causative studies are urgently needed to evaluate this possible correlation and, if found, to develop appropriate interventions to reduce survivors' cancer risk. Objectives: The participant should be able to identify two ways human trafficking may potentially increase cancer risk. References: Holman, D. M., et al. "The Association Between Adverse Childhood Experiences and Risk of Cancer in Adulthood: A Systematic Review of the Literature." Pediatrics, vol. 138, no. Supplement, 2016, doi:10.1542/peds.201542681. Stoklosa, Hanni, et al. "Medical Education on Human Trafficking." AMA Journal of Ethics, vol. 17, no. 10, 2015, pp. 914 921., doi:10.1001/journalofethics.2015.17.10.medu1-1510.
P21: Examining the effects of the Ketogenic Diet on the Nutritional and Psychosocial Status of Cancer patients in a Small Island Developing State: A case control study

Eden Augustus

The University of the West Indies, Cavehill Campus, Barbados, WI, Arima, Arima, Trinidad and Tobago

Abstract: Cancer cells rely on glucose to survive. Although the ketogenic diet (KD), high fat, low carbohydrates, have been deemed an adjuvant therapy. Sparse research exists on differential effects. This study aims to stregthen the understanding of the effects of the KD diet on factors of quality of life among cancer patients. Case control study. Twenty (20) stage $2 / 3$ cancer patients receiving chemotherapy and 20 cancer-free persons were recruited from an online survey testing KD awareness. Interested adults, that met the eligibility criteria were stratified and randomized by age, gender and medical history. A diet encompassing $10 \%$ carbohydrates, $15 \%$ proteins and $75 \%$ fats (2000 calories) was administered during a 16-week period. Anthropometrics and blood were collected by trained nurses after an overnight fast at baseline and weeks 2, 6, 12 and 16. Independent samples t-tests and repeated measures were used and presented as means and standard deviations.Ethical approval was gained. No significant differences ( $p>0.05$ ) were observed for nutritional and biochemical measures at baseline between groups. Differences were seen at baseline for psychosocial status scores with the cancer patients having significantly lower scores $(22.65 \pm 4.99)$ compared to the control group (26.40 \pm 5.59$)$.Significant differences in trends with control group versus the cancer patients were observed across the study time for BMI $[\mathrm{p}=0.042 ; \mathrm{d}=0.11$; small effect size $)$ and psychosocial health $(\mathrm{p}=<$ $0.001, d=0.733$, large effect size) Findings suggest that the KD may not have negative nutritional effects on cancer patients, in fact it may improve nutritional status, and mental health status (psychosocial). The study revealed significant improvement in BMI and psychosocial scores in cancer patient. It also revealed a keto-adaption period of seven to ten days. Objectives: The participant shall be able to determine the effects that the ketogenic diet has on the nutritional status of cancer patients. The participant shall be able to determine the effects that the ketogenic diet has on the psychosocial (mental health) status of cancer patients. References: Bray, F., and M. Pineros. 2016. "Cancer patterns, trends and projections in Latin America and the Caribbean: a global context." Salud Publica Mex no. 58 (2):104-17. Vergati, M., E. Krasniqi, G. D. Monte, S. Riondino, D. Vallone, F. Guadagni, P. Ferroni, and M. Roselli. 2017. "Ketogenic Diet and Other Dietary Intervention Strategies in the Treatment of Cancer." Curr Med Chem no. 24 (12):1170-1185. doi: 10.2174/0929867324666170116122915.

P22: The Lippincott Resource and Education Center: An Innovative Approach to Providing Effective Cancer Education and Awareness

\section{Nina Galpern, Leah Powers, Stephanie Raivitch, Evelyn Gonzalez Fox Chase Cancer Center, Philadelphia, PA, USA}

Abstract: The Lippincott Resource and Education Center (REC) was established at Fox Chase Cancer Center in 2000 to address the need for access to reliable information across the cancer continuum. The REC is a multi-media patient and family learning center providing education and referrals to credible sources. A program of the Office of Community Outreach, the REC has consistently evolved to meet the diverse needs of over 66,000 patients, caregivers, and the public who have visited. REC staff are trained educators with backgrounds in health education and research. Interactions are personally tailored to meet individual requests. They focus their efforts on the program's objectives to provide: evidencebased cancer information in a health literate manner, internal and community referrals, support to cancer patients and caregivers through the Patient to Patient Network (P2PN), and participation in research endeavors. The REC also supports community outreach activities by 
developing plain language, bilingual resources. Over 300 people a month visit the REC. The program addresses social determinants of health by acting as a hub to connect patients (50\%), caregivers and family (42\%) and the public to critical resources and referrals to healthcare, financial, insurance, transportation, housing and psychosocial services. One such service is the P2PN. This peer mentoring program has trained 200 volunteers and matched over 100 patients and caregivers. In response to the Community Health Needs Assessment (CHNA), the REC added monthly education sessions on topics including nutrition, genetics, and spirituality, led by faculty and staff. The 2016 Community Health Needs Assessment (CHNA) revealed a need for preventive cancer education, access to care, supportive services, and health literacy issues. REC satisfaction surveys show that $82 \%$ of visitors found REC information helpful. We will share information regarding the evolution of programs and data collection practices. Objectives: Following this presentation, the participant shall be able to describe three key functions to developing a Resource Center to address social determinants of health. References: Peer Mentors for People with Advanced Cancer: Lessons Learnt from Recruiting and Training Peer Mentors for a Feasibility Randomized Controlled Trial. Walshe C, et al. J Cancer Educ. 2020. A Prospective \& PopulationBased Inquiry on the Use and Acceptability of Peer Support for Women Newly Diagnosed With Breast Cancer. Legg, M et al. Support Care Cancer, 2019 Feb;27(2):677-685. What information do cancer patients want and how well are their needs being met? Chua GP et al. Ecancermedicalscience. 2018 Sep 25;12:873. Patients and caregivers' unmet information needs in the field of patient education Truccolo I Support Care Cancer. 2019 Jun;27(6):2023-2030.

P23: Standardizing Patient Education to Increase Distribution and Use, Inform Patients and Caregivers, and Reduce Unplanned Use of Clinic and Emergency Department for Symptom Management

Karen Arnold-Korzeniowski ${ }^{1}$, Christina Bach ${ }^{1}$, Carolyn Vachani ${ }^{1}$, Marisa Healy ${ }^{1}$, Tammi Fisher ${ }^{2}$, Gina Wierzbowski ${ }^{3}$, Amy Kyriakos ${ }^{3}$, Margaret Hampshire ${ }^{1}$, Benjamin Goldsmith ${ }^{2}$, Christine Hill-Kayser ${ }^{1}$, James Metz $^{11}$, Isabella Amaniera ${ }^{1}$

${ }^{1}$ OncoLink, Philadelphia, PA, USA, ${ }^{2}$ University of Pennsylvania Health System, Cherry Hill Radiation Oncology, Cherry Hill, NJ, USA, ${ }^{3}$ Doylestown Hospital, Department of Radiation Oncology, Doylestown, PA, USA

Abstract: Many centers lack resources/ infrastructure to provide standardized educational materials to cancer patients; in addition, many public cancer education resources are no longer kept current and freely available. Here, we evaluate the role of a standardized nursing-led approach to patient education, including detailed written materials, in care of radiation oncology patients. Patients were recruited from two radiation oncology centers affiliated with the same academic center. Center 1 used a standardized education approach of a nurse giving written materials to the patient at start of treatment; Center 2 did not use standardized education materials / delivery. Patients enrolled consecutively at each center were asked to complete a survey regarding impact of educational materials. Center 1 used materials from the OncoLink Treatment Binder Builder (OncoLink.org). All procedures approved by the IRB. Patients: 150 from C1 (Center 1) (median age 67y, 35\% male); 81 from C2 (Center 2) (med age $67,49 \%$ male). Diagnoses included breast ( $45 \%$ vs $26 \%$ ), prostate (9\% vs 30\%), lung cancer (14\% vs $12 \%$ ). Most patients read the materials $(\mathrm{p}=0.4)$. $\mathrm{C} 1$ patients always received materials from providers ( $\mathrm{p}$ Provision of standardized cancer patient education results in increased use, reducing need to contact HCT and unplanned/ emergency visits. In a non-standardized center, most patients obtain and read educational materials, suggesting value perceived by providers and patients. Future efforts should focus on standardization of patient education during cancer treatment. Objectives: The participant shall be able to identify two measurable outcomes (such as calls to providers and unplanned clinic visits) of implementing standardized education processes for patients receiving treatment for cancer. References: Arya, R., Zhu F., \& Golden, D. W. Patient Educaion - We Know We Need It, But Now What? International Journal of Radiation Oncology. 2017. 99(2) 121. Olivier H., Mukeshimana V., Ahishakiye A., Makuza P., Hategekimana V., Muhayimana C, et al. Standardization of Education of Patients With Cancer in a Low- and Middle-Income Country: A Quality Improvement Project Using the Cancer and You Booklet. Journal of Global Oncology. 2019. 5, 1-6. Rooney, M.K., Sachdev, S., Byun J., Jagsi, R., \& Golden, D. W. Readability of Patient Education Materials in Radiation Oncology Are We Improving? Practical Radiation Oncology. 2019. 9(6)

\section{P24: Cost-Effectiveness Analysis of Noninvasive Screening Tests for} Colorectal Cancer according to Race and Ethnicity

Xiao Zhang, Sandra Suther, Matthew Dutton, Askal Ali

Florida A\&M University college of pharmacy-economic, social, and administrative pharmacy (ESAP), Tallahassee, FL, USA

Abstract: Noninvasive screening tests for colorectal cancer (CRC) have limited complications and high adherence rates. This study aimed to simulate the clinical and economic benefits of two noninvasive screeing tests, the fecal immunohistochemical test (FIT) and multitarget stool DNA test (Mt-sDNA), among different races and ethnicities in the United States. A Markov model from a US payer (Medicare) perspective was constructed to compare the cost-effectiveness of the noninvasive tests for 50-year-old hypothetical cohorts of non-Hispanic white, non-Hispanic black, Hispanic, or Asian. The model continued 25 years. Markov health states categorized into: normal, polyp, local to distant CRC and death. The strategies included annual FIT, 3-yearly Mt-sDNA and necessary colonoscopy. Prevalence rates, transition probability, cost, qualityadjusted life years (QALYs) were collected from publications. Survival rates, adherence on follow-up and sensitivity of screening were estimated from the public database. A 3\% discount rate, deterministic and probabilistic sensitivity analysis were performed. Over the 25 -year period, the incremental cost-effectiveness ratios (ICERs) for the annual FITstrategy versus the 3 yearly Mt-sDNA strategy were the following: \$26,313/ QALY (non-Hispanic white), \$30,003/QALY (non-Hispanic black), $\$ 31,810 /$ QALY (Hispanic) and \$33,513/QALY (Asian). At willingness-to-pay threshold $\$ 50,000 / \mathrm{QALY}$, the annual FIT strategy was cost-effective for non-Hispanic white, non-Hispanic black, Hispanic and Asian. In the deterministic sensitivity analysis, the model was sensitive to the cost of Mt-sDNA, the cost of local cancer treatment, the transition rate from local to regional cancer, and sensitivity of MtsDNA test. Probabilistic sensitivity analysis showed the robustness of the result. Although the annual FIT was more expensive than the three-yearly Mt-sDNA, the annual FIT was more effective than the three-yearly MtsDNA in lowering colorectal cancer cases. The annual FIT could be used as the primary method for CRC screening in non-Hispanic whites, nonHispanic blacks, Hispanics and Asians aged 50 years. Objectives: Identify the economic and clinical benefits of using noninvasive colorectal cancer (CRC) screening among different races and ethnicities. Through analyzing cost-effectiveness of noninvasive CRC screening tests, high-risk races and ethnicities would be able to acknowledge costs and quality-adjusted life years (QALYs) of adhering the tests. Additionally, policy makers or third-party payers would be informed the primary CRC strategy for the specific race or ethnicity. A Markov simulation model would be constructed to estimate the incremental costeffectiveness of two noninvasive screening strategies (annual FITstrategy versus the 3 yearly Mt-sDNA strategy). References: Wilson, F. A., Villarreal, R., Stimpson, J. P., \& Pagán, J. A. (2014). CostEffectiveness Analysis of a Colonoscopy Screening Navigator Program Designed for Hispanic Men. Journal of Cancer Education, 30(2), 260 267. doi: 10.1007/s13187-014-0718-7. Sharma, T. (2020). Analysis of the effectiveness of two noninvasive fecal tests used to screen for colorectal cancer in average-risk adults. Public Health, 182, 70-76. doi: 10.1016/j.puhe.2020.01.021 
P25: Electronic Cigarette Marketing's Influence over the LGBTQ Community's Tobacco Usage

Michael Skipworth, Georgia Robins Sadler, France Nguyen-Grozavu, Matthew Stone

UCSD Moores Cancer Center, La Jolla, CA, USA

Abstract: The LGBTQ community has been targeted by tobacco advertising. Early initiation and increased prevalence of tobacco use contribute to prolonged consumption in this population, thereby increasing morbidity and mortality risks. Using the structural model of health, this literature review explores the influence of electronic cigarette advertisements on LGBTQ communities. The scientific literature was searched for publications that evaluated the influence of electronic nicotine delivery systems (ENDs) marketing on product awareness and tobacco use behavior among young adult LGBTQ communities. PubMed, CINHAL, and PsycINFO databases were searched using combinations of such major search terms as marketing, electronic nicotine delivery systems, sexual and gender minorities. Reference articles were also reviewed to identify additional articles related to the content and theme areas such as mode of ad delivery, product awareness, and behavior influence. Papers were eligible if published in peer-reviewed journals from 2012 to 2020 and excluded if inaccessible in full text. The Masters Settlement Agreement which placed significant restrictions on tobacco advertising, marketing, and promotional programs, should have reduced the tobacco companies' targeted promotion of ENDS to the LGBTQ community. However, twenty-two articles were identified in which END products appeared on both television and social media sites, including Twitter, Facebook, and Snapchat. Compared to their straight counterparts, LGBTQ users of these mediums were more likely to be exposed to pro-tobacco content, while also less likely to be exposed to anti-tobacco content. Additional literature discovered that advertisements on these platforms increased both awareness and use of ENDS among the LGBTQ community. Despite the 2018 deeming rule updating regulatory policies to include advertising restrictions for ENDS, marketing for these products continues. ENDS advertising targeting LGBTQ communities is ongoing, and violations should be monitored with punishments enforced. LGBTQ populations would benefit from public health advertising promoting tobacco-free lifestyles and cessation interventions. Objectives: The participant will be able to identify current and potential ENDS marketing towards the LGBT community. References: Emery, Sherry L., et al. "Wanna Know about Vaping? Patterns of Message Exposure, Seeking and Sharing Information about eCigarettes across Media Platforms." Tobacco Control, vol. 23, no. suppl 3, Ju Emory, Kristen, et al. "Lesbian, Gay, Bisexual, and Transgender (LGBT) View It. Emory, Kristen, et al. "Lesbian, Gay, Bisexual, and Transgender (LGBT) View It Differently Than Non-LGBT: Exposure to Tobacco-Related Couponing, E-Cigarette Advertisements, and AntiTobacco Messages on Social and Traditional Media." Nicotine \& Tobacco Research, vol. 21, no. 4, Mar. 2019, pp. 513-22. DOI.org (Crossref), doi:10.1093/ntr/nty049.

\section{P26: Providing Education to Patients Virtually: The Digital Education Prescription}

Naa Kwarley Quartey, Vincenzo Addario, Jeffrey Cardozo, Meredith Giuliani, Janet Papadakos

Princess Margaret Cancer Centre, Toronto, ON, Canada

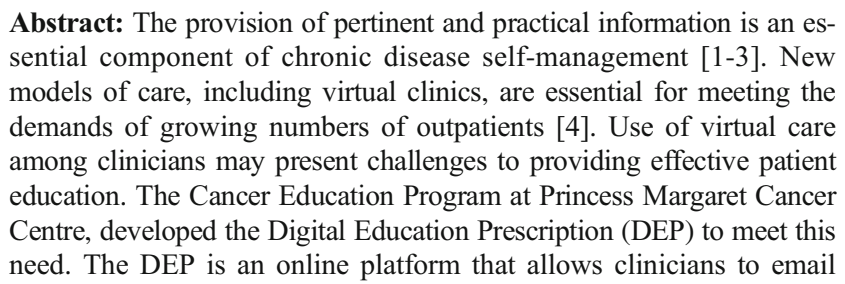

patients educational resources safely and securely. Approximately 2,000 resources, including pamphlets, links to online classes, videos and chemotherapy patient information sheets normally handed out to patients during in person clinic appointments are available in the DEP. The DEP consists of over 30 clinic profiles with resource collections that are tailored for each clinic to meet the unique information needs of patients within the clinic. Over 700 individual emails, consisting of more than 1,300 resources, have been sent to patients and caregivers through the DEP during the 4-month period since launch. To ensure resources are current and patients receive up to date information, the DEP is integrated within a content management system and links to external resources are checked using a link validator. To date, 5 virtual cognitive interviews have been conducted with clinicians who prescribe education through the DEP to gain insight into how the DEP has been integrated as part of virtual care and to gather feedback to improve the overall user experience. The DEP has increased the ability for clinicians to deliver education to patients through virtual means and has become an essential part of virtual care. Clinician access to the DEP ensures patients can continue to receive education to increase disease self-management and improve overall health outcomes. Objectives: The participant shall be able to describe how to effectively integrate digital education into virtual clinic visits. References: [1] Institute of Medicine. 2003. Priority Areas for National Action: Transforming Health Care Quality. Washington, DC: The National Academies Press. https://doi.org/10.17226/10593. [2] van de Bovenkamp HM, Dwarswaard J. The complexity of shaping selfmanagement in daily practice. Health Expect. 2017;20:952-960. [3] Ellis J, Boger E, Latter S, et al. Conceptualisation of the 'good' selfmanager: a qualitative investigation of stakeholder views on the selfmanagement of long-term health conditions. Soc Sci Med. 2017;176: 25-33. [4] Mehrotra A, et al. 2020. Rapidly Converging to "Virtual Practices": Outpatient care in the Era of Covid-19. NEJM Catalyst. April 1, 2020.

\section{P27: Publication Impact of a Short-term Summer Cancer Research Internship Program}

Luz Padilla $^{1}$, John Waterbor ${ }^{1}$, C. Michael Brooks ${ }^{3}$, Haley Greene ${ }^{4}$ ${ }^{1} U A B$ School of Public Health, Birmingham, AL, USA, ${ }^{2} U A B$ Schools of Medicine \& Health Professions, Birmingham, Alabama, USA, ${ }^{3} U A B$, Birmingham, Alabama, USA

Abstract: Published work that results from research conducted during short-term research training programs serves as an outcome measure for the author's success and the program's value. Little is known about the scientific impact one should expect from publications that result from such students and programs. The purpose of this report is to compare the impact of publications from research conducted during a short cancer research program to papers published by the same students after they have completed the training program and earned an academic or professional degree. Bibliometrics and altmetrics from NIH, ResearchGate, Google Scholar and Scopus ${ }^{\circledR}$ determined to be the best measures of publication impact were collected on publications from trainees and alumni of the CaRES (Cancer Research Experiences for Students) program from 19992019. Publication characteristics and impact measures for papers by CaRES interns and alumni were described and compared using SAS 9.4. Of 558 publications, $206(37 \%)$ resulted from CaRES internships. These publications were cited more frequently and held a higher research interest score than cancer articles published later by CaRES alumni yet appeared in journals with lower impact factors $(p<0$.05). A higher proportion of alumni were first author of their publication when compared to interns ( $\mathrm{p}$ 0.0213). Although publication characteristics vary between CaRES interns and alumni, research conducted during short-term cancer research experiences can be scientifically impactful and is comparable to that of program alumni who engage in cancer research careers. Objectives: The participant shall be able to identify at least two metrics that measure impact for cancer publications. References: Alamri, Y., 
Currie, W., Magner, K., Al-Busaidi, I. S., Wilkinson, T. J., \& Beckert, L. (2019). Publication rates of, and attitudes toward, summer research projects: 10-year experience from a single institution in New Zealand. Advances in medical education and practice, 10, 263-271. DOI:10.2147/AMEP.S198789. Desmond, R. A., Padilla, L. A., Daniel, C. L., Prickett, C. T., Venkatesh, R., Brooks, C. M., \& Waterbor, J. W. (2016). Career Outcomes of Graduates of R25E Short-Term Cancer Research Training Programs. Journal of Cancer Education, 31(1), 93100. DOI:10.1007/s13187-014-0786-8.

\section{P28: Implementing a pediatric resident curriculum to improve con-} fidence with delivering bad news to patients and families

Hatai Kraushaar ${ }^{1}$, Douglas Ross ${ }^{2}$, Nan Garber $^{3}$

${ }^{1}$ Sinai Hospital, Gig Harbor, WA, USA, ${ }^{2}$ University of Maryland, Baltimore, MD, USA, ${ }^{3}$ University of Maryland Medical Center, Baltimore, MD, USA

Abstract: The ability to effectively and compassionately deliver bad news is an essential skill for all physicians, yet many pediatric residency programs do not provide formal instruction on the subject. Trainees are often expected to develop communication skills independently through observation and trial and error, with varying results. The goal of this study is to pilot a curriculum for senior pediatric residents rotating in the pediatric intensive care unit to improve confidence with delivering bad news. Residents received an interactive didactic and implemented what they learned by disclosing bad news in a real family meeting. Changes in comfort with the above task were assessed by pre- and post-curriculum questionnaires. To evaluate the impact of adding simulation training, residents were randomized to one of two arms, one with and one without simulation. The simulation residents practiced delivering bad news to an actor parent prior to leading a real family meeting. Confidence with delivering bad news increased significantly across all residents as a result of the curriculum ( $\mathrm{p}=0.001)$. Residents who also received simulation training $(n=9)$ trended towards a greater increase in confidence with respect to the control group $(n=7 ; p=0.1)$. Confidence improved the most in residents who were the least confident at the start of their rotation $(\mathrm{p}=0.002)$. Resident feedback on the curriculum and simulations was overwhelmingly favorable, and both groups felt simulation training was important and should be a part of the final curriculum. Delivering bad news can be intimidating and stressful, but this curriculum significantly increased resident confidence with this important skill. Participant feedback suggests the curriculum was meaningful and addressed an important unmet educational need. A larger sample size is needed to clarify the effect of simulation on confidence. Objectives: The participant should be able to recognize the need for formal residency training in delivering bad news and the potential positive impact of this training on resident confidence. References: Wolfe AD, Denniston SF, Baker J, Catrine K, HooverRegan M. Bad news deserves better communication: a customizable curriculum for teaching learners to share life-altering information in pediatrics. MedEdPORTAL. 2016;12:10438. Servotte JC, Bragard I, Szyld D et al. Efficacy of a short role-play training on breaking bad news in the emergency department. West J Emerg Med. 2019;20(6)893-902. McCallister JW, Gustin JL, Wells-Di Gregorio S, Way D, Mastronarde JG. Communication curriculum for pulmonary and critical care fellows. AnnalsATS. 2015;12(4)520-525.

\section{P29: Does Radioactive Diagnostic Testing in Infants Increase their} Risk for Developing Adolescent Cancer?

Anthony Cirilo, Juan Andrew Leal, Gavrila Ang, Suzi Hong, France Nguyen-Grozavu, Georgia Robins Sadler

${ }^{1}$ UCSD Moores Cancer Center, La Jolla, CA, USA

Abstract: Causes of cancer in adolescents are less investigated compared to other age groups. Scientific literature has implicated that ionizing radiation from diagnostic tests causes tissue damage. Infantile tissue is delicate and highly susceptible to damage. This review investigated diagnostic tests conducted during infancy as a contributor to adolescent cancer development. This review examined evidence from the scientific literature that compared rates of radiation exposure, absorbance, and cancer rates to understand the role radioactive diagnostic testing plays in adolescent cancer development. It also aimed to uncover any disparities in rates of cancer development in adolescents who underwent diagnostic radiologic tests during infancy compared to adolescents who did not. Key databases searched included PubMed/Medline, PsycInfo, CINAHL, Embase, and Google Scholar for the articles published in and after 1980. Keywords used for the search included: diagnostic detection, infant, adolescent, young adult, CT scans, cancer, cancer prevalence, radiation absorbance, dosage, and childhood cancer. The literature provided indirect evidence for the cancer-infant radiation link. The cancer rates of adolescents and young adults, roughly 13-26 years of age, have increased by $25 \%$ over the last three decades. Radiation exposure through recent medical practices have also increased, accounting for $50 \%$ of American's average annual radiation exposure. In CT scans, a key medical radiation source, parameters are typically unadjusted between adults and children. With a higher radio-sensitivity, infantile tissue has an increased susceptibility to irreparable tissue damage and DNA mutations. This susceptibility is hypothesized to predispose these infants to cancer development in comparison to non-radioactively tested infants. While the literature suggests a correlation between radioactive testing and cancer development in children, no direct evidence corroborates this link. Studies are needed to fully understand this phenomenon. Proper radiation dosage provides a feasible method to encourage safer testing during childhood, and parents need this information to guide clinical decision-making. Objectives: The participant shall be able to understand the risks that come with radiation exposure for infants, including cancer development into adolescence and young adulthood. References: Bernier, M., Rehel, J., Brisse, H. J., WuZhou, X., Caer-Lorho, S., Jacob, S.,... Laurier, D. (2012). Radiation exposure from CT in early childhood: A French large-scale multicentre study. The British Journal of Radiology, 85(1009), 53-60. oi:10.1259/ bjr/90758403.

Miglioretti, D. L., Johnson, E., Williams, A., Greenlee, R. T., Weinmann, S., Solberg, L. I.,... Smith-Bindman, R. (2013). The Use of Computed Tomography in Pediatrics and the Associated Radiation Exposure and Estimated Cancer Risk. JAMA Pediatrics, 167(8), 700. doi:10.1001/ jamapediatrics.2013.311

P30: Head and Neck Cancer Registry of an Underserved Urban Population Generated from Multi-Disciplinary Tumor Board Presentation

James Hamilton $^{1}$, Charles Moore ${ }^{2}$, Oswaldo Henriquez ${ }^{2}$

${ }^{1}$ Emory University School of Medicine, Atlanta, GA, USA, ${ }^{2}$ Emory University Department of Otolaryngology, Atlanta, GA, USA

Abstract: Multidisciplinary tumor boards (MDTB) are associated with changes in staging and management, higher rates and shorter time to treatment after diagnosis, and adherence to clinical guidelines1. Evidence shows they improve survival outcomes and inter-disciplinary cooperation2. Details of MDTB decisions are recorded inconsistently, and often miss salient discussion points3.The majority of providers have insufficient access and infrastructure to accurately assess the number of patients discussed annually, the total tumor caseload, and the proportion of newly diagnoses 2 . We aim to structure a secure, web-based, customizable instrument for head and neck cancer MDTB presentation within a large, urban, publicly-funded hospital. It will prompt users for mandatory datapoints regarding demographics, staging, histopathology, MDTB discussion/decision, and treatment/compliance. Our goal is uniform presentation, direct Electronic Medical Record integration for rapid access to data, and a repository for oncologic data for research and audit specific to an underserved population consisting largely of racial/ethnic minorities 
and underinsured patients3. The proportion of patients receiving guidelinebased care has decreased, and patients with lower socioeconomic status who receive care in safety-net settings regardless of insurance status or ability to pay are most vulnerable4. This model provides capability for audit of aspects of MDTB decisions, compliance /outcomes tracking, and comparison with other national/international databases to ensure standard of care delivery. There is a fundamental need for oncologic data banks particularly with a focus on low-income and racial/ethnic minority populations. This population is disproportionately cared for in safety-net settings and is at increased risk for delays in care delivery and lapses in monitoring. Complete, uniform data can augment the ability to assess whether MDTB recommendations align with best practice and identify areas for improvement. The growing registry will aggregate data for new research. Data can be easily exported into statistical software for audit or research. This platform can be translated across oncologic specialties. Objectives: The participant shall be able to recognize the utility of the implementation of an electronic, web-based MDTB framework that generates a uniform comprehensive tumor board presentation for each patient and its value as a resource in the aggregation of accurate oncologic data collection for research and audit purposes specific to a high-risk underserved population. References: Pillay B, Wootten AC, Crowe H, et al. The impact of multidisciplinary team meetings on patient assessment, management and outcomes in oncology settings: A systematic review of the literature. Cancer Treatment Reviews. 2016;42:56-72. Rankin NM, Lai M, Miller $\mathrm{D}$, et al. Cancer multidisciplinary team meetings in practice: Results from a multi-institutional quantitative survey and implications for policy change. Asia-Pacific Journal of Clinical Oncology. 2018;14(1):74-83. Rangabashyam M, Wee HE, Wang W, et al. Electronic tumor board presentations as the basis for the development of a head and neck cancer database. Laryngoscope Investigative Otolaryngology. 2020.

\section{P31: Web Design in Cancer Care: Rethinking how the web can best serve the needs of diverse audiences}

\section{Nicole Liscio, Janet Papadakos, Meredith Giuliani, Tina Papadakos Princess Margaret Cancer Centre, Toronto, ON, Canada}

Abstract: The website of a cancer centre is often a primary point of contact for patients or people interacting with the centre in any capacity. The Princess Margaret Cancer Centre's website is 7 years old and due for a significant update. The average life span of a website should be no more than 2-3 years to ensure that it is keeping up with current standards, user needs, and the goals of the organization $[1,2]$. A current state assessment is planned to identify the main audiences (user groups), the needs of users, and how the website is/is not meeting those needs. Goals of the assessment: Establish baseline metrics to benchmark annual website performance. Assess gaps in content and design from a health literacy and accessibility perspective. Establish a long-term web improvement strategy, including recurring evaluation and reassessment of look and feel The Current State Assessment includes: Environmental scan to better understand the current landscape of websites from healthcare and other industries, as well as web best practices [1,2]. Baseline assessment of Princess Margaret website. Data will be collected using Google Analytics and surveys. Health literacy scoring tools will establish the website's suitability for those with low health literacy [3]. User experience and usability tests will be performed to learn how different users interact with the website and to find opportunities for improvement. Findings will be used to plan and implement large-scale improvements to improve the overall experience of the website. A key resource for the public, the Princess Margaret website must meet its users' needs. The website can be used to promote trusted information and improve health outcomes. Assessment data will help clarify user demographics, focus improvements, and ensure that users can find what they need when they need it. Objectives: The participant shall be able to: Describe a process for performing an environmental scan and baseline assessment for the purposes of improving digital products (like websites). Describe high level findings from a needs assessment and reflect on potential actions that could be taken to address any relevant gaps or findings. References: [1] What is the average website lifespan? Orbit Media Studios. [2] Why Companies Redesign Websites Every Three Years on Average - Or Do They? Walker Sands Digital. [3] Shoemaker SJ, et al. Development of the Patient Education Materials Assessment Tool (PEMAT): a new measure of understandability and actionability for print and audiovisual patient information. Patient Educ Couns 2014;96(3):395-403. [4] Ngyuen MH, et al. Optimising eHealth tools for older patients: Collaborative redesign of a hospital website. Eur J Cancer Care. 2019;28(1):e12882. [5] Faller H, et al. Satisfaction with information and unmet informaiton needs in men and women with cancer. CJ Cancer Surviv. 2016;10(1):62-70.

\section{P32: Cancer Classes Online: A New Site for Digital Patient Education}

Tina Papadakos, Tylar Stringer, Matt Turczyn, Sandra Clarke, Vincenzo Addario, Sarah Storer, Meredith Giuliani, Janet Papadakos Princess Margaret Cancer Centre, Toronto, ON, Canada

Abstract: eLearning as mode of education has advantages including increased access, standardized content, and options to personalize pace. eLearning also permits the widespread distribution of patient education content to users with geographic, economic, or logistical barriers. Prior to the COVID-19 pandemic, in-person classes at Princess Margaret were made available via eLearning. During the pandemic, with efforts to reduce in-person visits, including cancellation of in-person classes, eLearning usage before and after lockdown in Toronto, increased by $160 \%$. Cancer Classes Online (PMCancerClasses.ca) was launched in September 2019 to host multimedia classes for cancer patients and public to access anytime, anyplace and on any device. Over 45 classes are available in plain language and plain design to maximize understanding and user experience. Since launch, 400 responses collected from live quality improvement surveys embedded in each class show rates of satisfaction ranging from $96-100 \%$ on the ease of understanding content, content relevance and usability. The platform is continuously monitored and evaluated through four main mechanisms to meets user's needs. These include: 1. Usage data and analytics including number of accounts, courses completed by users, and geographic reach of the platform, 2. Mini quality improvement surveys embedded into each class to track patient satisfaction and ideas for patient-led improvements, 3. A salient belief question at the end of the class that asks users what is on their mind now in order to predict new or emergent questions or needs. 4. An research study comparing in-person classes with online classes on a number of factors including access and equity. Cancer Classes Online allows patients and public to access health literate patient education content anytime, anywhere, alleviating geographic and logistical barriers of coming to in-person classes, this has been particularly important during the COVID pandemic. Objectives: The participant will be able to describe the current impact of a new digital education platform designed to provide health literate cancer education for patients and the public to access anytime, anywhere. The participant will be able to describe evaluation mechanisms for online learning. References: Samantha Ayoub, Edmund Tsui, Taariq Mohammed \& Joseph Tseng (2019) Readability Assessment of Online Uveitis Patient Education Materials, Ocular Immunology and Inflammation, 27:3, 399-403, DOI: 10.1080/09273948.2017.1413396. Win, K.T., Hassan, N.M., Oinas-Kukkonen, H. et al. Online Patient Education for Chronic Disease Management: Consumer Perspectives. J Med Syst 40, 88 (2016). https://doi.org/10.1007/s10916-016-0438-0.

P33: Reducing cervical cancer health disparities among females living with HIV: Is there an app for that?

Lisa Tisdale Wigfall ${ }^{1}$, Otis (Shaun) Owens ${ }^{2}$, Aditi Tomar ${ }^{1}$, Erica Collins ${ }^{1}$, Tian Chen ${ }^{1}$

${ }^{I}$ Texas A\&M University, College Station, TX, USA, ${ }^{2}$ University of South Carolina, Columbia, SC, USA 
Abstract: Females living with HIV (FLWH) are 3-8 times more likely to be diagnosed with cervical cancer. Although HPV vaccination and cervical cancer screening apps exist that can reduce these health disparities, little is known about their use among FLWH. We describe FLWH's mHealth use and cervical cancer prevention app preferences. Eightyeight females at community-based HIV/AIDS service organizations (ASOs) completed our 76-item self-administered, web-based survey in August 2020. In addition to assessing app use and preferences, we also assessed age, race/ethnicity, sexual orientation, and cervical cancer risk factors. Smartphone or tablet owners were asked about their current app use (tracking health-related goals, making medical decisions), and preferences for a comprehensive cervical cancer prevention app that would target HPV vaccination and Pap/HPV testing. Sample characteristics, current app use, and willingness to use an app to increase HPV vaccination and cervical cancer screening rates among females at ASOs are described. Our sample was $35.2 \pm 12.6$ years (range: $18-72$ ); $34 \%$ Black, $31 \%$ Hispanic/LatinX, 23\% Asian, 12\% White; $83 \%$ straight/heterosexual, $5 \%$ lesbian, $10 \%$ bisexual, $6 \%$ asexual/aromantic/pansexual/queer. Cervical cancer risk factors included HIV infection (18\%), long-term oral contraception use (31\%), and high parity (25\%). Smartphone/tablet users $(96 \%)$ used an app to track health-related goals $(71 \%)$ and/or make medical decisions (25\%). Many would use an app to learn about HPV vaccination $(54 \%)$ and cervical cancer risk factors $(59 \%)$, track cervical cancer symptoms (63\%), track when HPV shots (49\%) and Pap/HPV test(s) were due (63\%), and receive appointment reminders (51\% HPV vaccination, $69 \%$ cervical cancer screening). Many FLWH are using healthrelated apps on their smartphone/tablet. More research is needed to improve our understanding of how to design an app that will increase HPV vaccination and cervical cancer screening rates among this vulnerable population. To this end, an app can reduce cervical cancer health disparities among FLWH. Objectives: The participant shall be able to: Describe app use among FLWH. Identify at least one HPV vaccination or cervical cancer screening app preferences of FLWH. References: Ruiz-López T, et al. FightHPV: Design and Evaluation of a Mobile Game to Raise Awareness About Human Papillomavirus and Nudge People to Take Action Against Cervical Cancer. JMIR Serious Games. 2019 Apr 8;7(2):e8540. Lam JU, et al. Human papillomavirus selfsampling for screening nonattenders: Opt-in pilot implementation with electronic communication platforms. Int J Cancer. 2017 May 15;140(10):2212-2219. Bert F, et al. Tecnologia e prevenzione nell'era della Mobile Health: quali applicazioni per gli screening oncologici? [Technology and prevention in the era of mobile health: applications for cancer screening programs]. Ig Sanita Pubbl. 2017 JanFeb;73(1):13-27. Italian.

\section{P34: Food safety videos: Keeping immunocompromised patients safe}

Karen Lawrie, Natasha Icliates, Daniela Fierini, Megan Morrison, Connie Ziembicki, Janet Papadakos

Princess Margaret Cancer Centre, Toronto, ON, Canada

\begin{abstract}
People with weakened immune systems are at higher risk of contracting food-borne illnesses, and face hospitalization and even death.1 Even when patients with weakened immune systems are aware of their susceptibility to infection, they are not aware of the risks to their health through routine food handling practices. 2 The aim of this project was to create a comprehensive video series to educate patients about the actions they need to take to prevent food-borne infections. We created nine micro-videos that provide practical information and visual demonstrations about safe food handling. These videos cover safe food handling practices from preparing to grocery shop to storing leftover foods and how to read "best before" labels. All videos followed patient education best practices, including providing compelling reasons for following food safety recommendations, use of plain language and clear design. Evaluations are currently underway with a sample of five immunecompromised patients to collect their thoughts on the videos using a
\end{abstract}

semi-structured interview guide. In addition, YouTube analytics data will be collected: number of views, watch time (how long the viewer watched the video), audience retention (what parts of the video were most watched) and playback location (whether your content is being watched within the organization's YouTube channel or elsewhere). Analysis of this data, along with the results of the patient interviews, will allow us to measure audience engagement and inform future video strategies. This video series is designed to empower patients and their family with the knowledge necessary to protect themselves from food borne infections, and lower the risk of serious complications. This series was also designed to deliver content in a manner that addresses is available to individuals with low functional health. Objectives: The participant will learn how to convey large amounts of information in a compelling manner that avoids cognitive overload in patients. The particpant will learn how to create patient education videos that follow the principles of health literate design. References: 1) Paden H, Hatsu I, Kane K, et al. Assessment of Food Safety Knowledge and Behaviors of Cancer Patients Receiving Treatment. Nutrients. 2019;11(8):1897. Published 2019 Aug 14. doi:10.3390/nu11081897. 2) Medeiros LC, Chen G, Hillers VN, Kendall PA. Discovery and development of educational strategies to encourage safe food handling behaviors in cancer patients. J Food Prot. 2008;71(8):1666-1672. doi:10.4315/0362-028x-71.8.1666

\section{P35: EDUCATION AND HEALTH: The Mobile Unit of Health Education}

\section{Gerson Lúcio Vieira ${ }^{1}$, Joana de Jesus de Andrade $^{2}$ \\ ${ }^{1}$ Cancer Hospital Barretos, Barretos, São Paulo, Brazil, ${ }^{2}$ University of São Paulo, Ribeirão Preto, São Paulo, Brazil}

Abstract: The study analyzed the Scientific Dissemination project contributions carried out through a Mobile Unit equipped with serious games "Missão Gênese" (UMMG) from Barretos Cancer Hospital. It sought to identify if there was any motivation, engagement and learning about cancer prevention and if the knowledge was shared in the participants community. It was an analytical observational cohort study with a exploratory descriptive quali-quantitative approach, with data from a group of twenty-nine students in eighth grade from a public school participating in the "Missão Gênese" experience. The data construction was carried out in two stages: the first was through twenty-nine semi-structured questionnaire and recording of 15 -second videos at the end of the experience with questions about the impressions and learning provided by the experience. The second stage was carried out one year after the first, through semistructured interviews with the same students and two teachers. Obtaining data from different sources has allowed its triangulation, enabling an investigation object multifaceted study. The analysis was carried out with theoretical support of the historical-cultural approach, generating the six units of analysis construction: Motivation - identified emotions and reasons caused by the experience ;Tension - created challenges for students to feel compelled to learn; Engagement - created or rescued self-care and quality of life values; Sharing - promoted the dissemination and knowledge reporting; Articulation with School Contents - articulated with the Elementary Education legislation in force in Brazil; Appropriation of Technology - used a language and method closer The UMMG experience has the potential to promote development and learning among the participating adolescents by positively interfering in their conduct. In addition, it contributes to the appropriation of concepts and construction of knowledge about self-care, health promotion, promoting the engagement and experience sharing with the people around them. Objectives: Identify whether the Mobile Genesis Mission Unit (UMMG) is able to raise knowledge and practices capable of cancer education dissemination to the less favored population. Analyze whether the participants (adolescents) after participating in the UMMG experience are able to identify concepts of quality of life, health promotion and cancer prevention in their realities. It seeks to identify whether the participants are able to act as multipliers of the appropriate information and concepts in order 
to positively interfere in the social determinants of the communities where they live. References: 1. BRAZIL. Ministry of Health. Health Care Secretariat. National Cancer Institute. Coordination of Prevention and Surveillance. The cancer situation in Brazil. Rio de Janeiro: INCA. 2016, p.114. 2. VYGOTSKY Obras Escogidas: Tomo 3 (Spanish Edition) (Spanish) Paperback - January 2, 2016

P36: A psychoeducational intervention to cancer patients, cancer survivors and caregivers to prevent adverse psychosocial outcomes related to natural disasters.

Gina Sanchez-Cruz ${ }^{1}$, Melissa Marzán-Rodríguez ${ }^{2}$, Julio Jimenez ${ }^{2}$, Liliana Hernandez-Martinez ${ }^{1}$, Arline Vela-Vidal ${ }^{1}$, Mary RodriguezRabassa $^{2}$, Lynnette Ruiz-Ortiz ${ }^{2}$, Eida Castro-Figueroa ${ }^{2}$

${ }^{1}$ PSHU-MCC Partnership, Ponce, PR, USA, ${ }^{2}$ Ponce Health Sciences University, Ponce, PR, USA

Abstract: Since 2017, Puerto Rico has experienced natural disasters such as Hurricane Maria, earthquakes, and, currently, the impact of the COVID-19 pandemic. Cancer patients are significantly affected by natural disasters, due to individual and structural barriers. These stressful conditions can increase risk of psychosocial disorders, including cancerrelated Post-Traumatic Stress Disorder. The objective of this study is to explore the acceptability and feasibility of providing mental health support via remote to community members affected by cancer who exposed to disaster events. Using the Community-based Disaster Mental Health Intervention, culturally adapted for the Puerto Rican population, a series of psychoeducational workshops were developed through remote modality. A total of 52 participants (cancer/survivors and caregivers) fromthe southwest area received the intervention (two sessions/each participant). Through Google Form links via email and text messages, all the participants answered: informed consent, sociodemographic questionnaire, the Psychosocial Disaster-Related Screening Test (Spanish version), as well as workshops' evaluation. Ninety percent $(90.2 \%)$ of participant were females; $45.1 \%$ were from the $45-54$ age group, and $55.0 \%$ reported a monthly income of $\leq \$ 500.00$. All participants completed the intervention, and $97 \%$ completed the questionnairesprimarily using their smartphones. The acceptability of workshops was rated as high and very high on all assessed dimensions. Most of the participants mentioned the importance of having these educational experiences to increase their awareness and identify coping strategies for prevent adverse psychosocial outcomes. The remote modality of psychoeducational support showed to be acceptable intervention for people affected by cancer and impacted by disaster experiences. It is especially, a feasible option for hard to reach population. Changes in symptoms of anxiety and depression are currently under study. Objectives: Identify strategies to reach cancer patients/survivor/caregivers using a remote modality to provide emotional support in disaster situations. References: James, L. E., Welton-Mitchell, C., Noel, J. R., \& James, A. S. (2020). Integrating mental health and disaster preparedness in intervention: a randomized controlled trial with earthquake and flood-affected communities in Haiti. Psychological medicine, 50(2), 342-352. https://doi.org/10. 1017/S0033291719000163. Beaglehole, B., Mulder, R. T., Frampton, C. M., Boden, J. M., Newton-Howes, G., \& Bell, C. J. (2018). Psychological distress and psychiatric disorder after natural disasters: Systematic review and meta-analysis. The British Journal of Psychiatry, 213(6), 716-722. https://doi.org/10.1192/bjp.2018.210

P37: E-learning Training Adaptation: SHADE UP! Brazil: Education about sun protection and skin cancer prevention

Rosa Aparecida ${ }^{1}$, Ester Regina ${ }^{1}$, Beatriz Carvalho ${ }^{1}$, Sara de Mattos ${ }^{1}$, Joilson Rodrigo ${ }^{1}$, Vinícius Vazquez ${ }^{1}$, Stephan Bognar ${ }^{2}$, Tanzib Hossain $^{3}$, Marya Ghazipura ${ }^{2,3}$, Gerson Lúcio Vieira ${ }^{1}$

${ }^{1}$ Cancer Hospital Barretos, Barretos, São Paulo, Brazil, ${ }^{2}$ New York Dermatology Group Foundation, New York, NY, USA, ${ }^{3}$ New York University Langone Health, New York, NY, USA
Abstract: Skin cancer is a public health crisis in Brazil. In 2019, almost 200,000 Brazilians were diagnosed with a skin cancer. To address this, the Hospital de Cancer de Barretos and the New York Dermatology Group Foundation created a partnership and developed SHADE UP! Brazil. The aim has been to deliver one of the largest public health programs in Brazil focused on skin cancer prevention and promotion of healthy sun protection habits. However, due to Covid-19 restrictions, the program has had to adapt its focus from the classroom to a virtual learning environment. SHADE UP! was designed to be delivered through in-classroom activities to 31 public schools in the region of Barretos, San Paulo in the 2020-2021 academic year. During the Covid-19 pandemic, SHADE UP! went from an in-classroom program to a virtual e-learning environment. Targeted schools selected 5 students and two teachers (one science and one physical education teacher) to represent their school online. Once all targeted schools were trained via Zoom on the 7 SHADE UP! training modules over a 7-week period, students continued their learning through a virtual world called Game School. Game School consists of three components: 1) games and quizzes, 2) social media campaigns (videos, podcasts, and art on different project themes), and 3) science videos focused on how to apply SHADE UP! in the real world from the concepts learned. Participants must complete each stage before moving on to the next one. Quantitative and qualitative tools (including online testing, data analysis, surveys, and virtual interviews) will be used to measure program impact. Once physical distancing restrictions are lifted, SHADE UP! will be brought back into the classrooms to directly engage with students. The two program delivery modalities (in-person and e-learning) will be evaluated against each other to determine if the program obtains the same desired outcomes through e-learning during the Covid-19 pandemic as it would during in-person education. The pilot project for the e-learning phase is still ongoing. SHADE UP! is directly reaching 240 SHADE UP! participants serving as ambassadors and thousands of additional students in the targeted schools. SHADE UP! Ambassadors will then take their knowledge on sun protection and skin cancer prevention to their communities to save lives. Objectives: The participant must be able to identify an appropriate and adapted project application methodology for e-learning, so that health education about skin cancer and sun protection is carried out. In addition, the participant must be able to identify methodologies for evaluating the project in two formats.. In addition, the participant must be able to identify methodologies for evaluating the project in two formats. References: Instituto Nacional de Câncer José Alencar Gomes da Silva. Estimativa 2020: câncer de câncer no Brasil. Rio de Janeiro, 2019. Ministério da Educação. Base Nacional Comum Curricular (Terceira Versão). Ministério da Educação, Brasília, DF: MEC, 2016a. Disponível em: $\mathrm{http} / /$ historiadabncc.mec.gov.br/documentos/bncc-2versao.revista.pdf. Acesso em: 2020-08-31.

P38: Virtual continuing health professional education: a scoping review

Courtney Cheng, Janet Papadakos, Rouhi Fazelzad, Ben Umakanthan, Meredith Giuliani

Princess Margaret Cancer Centre, Toronto, ON, Canada

Abstract: Virtual continuing medical education (CME) has become increasingly popular, but limited data exist regarding how this approach can mitigate disparities faced by health professionals. Therefore, we aim to synthesize the advantages and disadvantages of virtual CME and to establish the impact of this strategy on inequities that health professionals face. This scoping review was conducted according to the Joanna Briggs Institute protocol. A comprehensive literature search for papers published in any language from January 1991 to June 2020 was performed in 6 databases in consultation with an information specialist. Three investigators screened publications for eligibility, and an independent investigator resolved any conflicts. Eligibility criteria included empirical studies and editorials focused on physicians and related to post-certification medical 
education events delivered in any virtual format. Demographic data, including healthcare specialty, virtual education modality, reported advantages and disadvantages, and location, gender, and race of participants, were extracted from included publications and summarized. The literature search yielded 31,485 studies, of which 9,723 duplicates were removed. The remaining 21,762 articles underwent title and abstract screening and 21,199 were excluded. 563 articles remained for full-text review. Full-text screening is ongoing. The most commonly reported advantages and disadvantages of this intervention will be reported. From an equity perspective, our findings with respect to gender, location, race, and profession will be summarized. Additionally, findings regarding the disparities and inequities faced by cancer health professionals will be highlighted. As various organizers look to virtual methods for delivering CME postCOVID-19, it is an ideal opportunity to re-evaluate CME standards and explore the possibilities of an equitable future stage for virtual CME. By exploring this topic, we hope to improve CME programs and eliminate disparities faced by cancer health professionals. Objectives: The participant shall be able to identify at least two advantages and two disadvantages of virtual CME on inequities that health professionals face regarding gender, location, race, and profession. References: Salinas GD. CME effectiveness: utilizing outcomes assessments of $600+$ CME programs to evaluate the association between format and effectiveness. J Contin Educ Health Prof. 2015;35 Suppl 1:S38-9. Jeong D, Presseau J, ElChamaa R, Naumann DN, Mascaro C, Luconi F, et al. Barriers and Facilitators to Self-Directed Learning in Continuing Professional Development for Physicians in Canada: A Scoping Review. Acad Med. 2018;93(8):1245-54

\section{P39: Pivoting to a Virtual Summer Cancer Research Program For Underrepresented High School and College Students}

Brittany C. Michel, Karen Burns White

Dana-Farber Cancer Institute, Boston, MA, USA

\begin{abstract}
Early intervention programs have been shown to increase retention of underrepresented minorities (URMs) in STEM, with student self-efficacy and confidence positively associated with activities in research preparation, professional development, and mentorship. Given how critical these programs are to the retention of URMs in STEM, we sought to continue and enhance our research training program during the pandemic by rapidly pivoting to a virtual platform. The YES for CURE, a 3 -year cancer-related research training program, serves URM high school and college students. With onsite research environments temporarily closed, this summer's program included several virtual enhancements: informational interviews to provide exposure to the cancer research community, and literature reviews under faculty mentors and facilitated curriculum on experimental design in lieu of onsite research. Additionally, virtual scientific seminars were provided with URM speakers from across the nation focused on health inequities. Virtual programming met or exceeded the expectations of all surveyed students $(n=36)$, and students were satisfied with their overall experience $(4.5+0.8 ; 1=$ strongly disagree, $5=$ strongly agree). The virtual enhancements were rated as very useful career preparation for cancer-related research or health disparity fields. Students in their second summer compared their experience to traditional in-person programming, noting the online experience was "as good as" or "better" for learning to communicate in writing/ presentations and access to sessions with high caliber speakers, and "as good as" for generating interest in and learning about careers in cancer research. These data indicate it is possible to provide meaningful cancer research experiences for URMs on a virtual platform. This can be in the form of all-virtual programs or incorporating virtual elements to existing in-person programming, potentially increasing flexibility and access to opportunities to ultimately diversify the STEM and healthcare workforce. Objectives: The participant shall be able to identify strategies to enhance virtual research training programs targeted to high school and college
\end{abstract} students. References: Ghee, M., Keels, M., Collins, D., Neal-Spence,
C., \& Baker, E. (2017). Fine-tuning summer research programs to promote underrepresented students' persistence in the STEM pathway. CBE-Life Sciences Education, 15(3), ar28. Maton, K. I., Beason, T. S., Godsay, S., Sto Domingo, M. R., Bailey, T. C., Sun, S., \& Hrabowski, F. A., III (2016). Outcomes and processes in the Meyerhoff Scholars Program: STEM PhD completion, sense of community, perceived program benefit, science identity, and research self-efficacy. CBE_-Life Sciences Education, 15(3), ar48.

P40: Southeast Partnership for Improving Research and Training in Cancer Health Disparities (SPIRIT-CHD): Increasing Outreach and Engagement in Underserved Communities through the Cancer Research Education

Z'Kera Sims $^{1}$, Lakeshia Cousin ${ }^{1}$, Vivekka Suppiah ${ }^{1}$, Paula Gregory ${ }^{2}$, Ursula Pradeda-Martinez ${ }^{1}$, Cathy Meade ${ }^{1}$, Gwendolyn Quinn ${ }^{3}$, Vani Simmons ${ }^{1}$, Fern Tsien ${ }^{2}$, Arnold Zea ${ }^{2}$, Clement Gwede ${ }^{1}$

${ }^{1}$ Moffitt Cancer Center, Tampa, FL, USA, ${ }^{2}$ Louisiana State University Health Sciences Center, New Orleans, LA, USA, ${ }^{3}$ New York University, New York, NY, USA

Abstract: The Southeast Partnership for Improving Research and Training in Cancer Health Disparities (SPIRIT-CHD), an LSUHSCMoffitt Cancer Center collaboration, includes a Cancer Research Education Program (CREP) focused on training underrepresented undergraduate and medical students in biobanking, precision medicine, and cancer health disparities. One component includes a community engagement seminar together with tailored experiences where trainees relay cancer prevention information to lay audiences. These practices aim to increase trainee self-efficacy in delivering scientific information to underserved communities. The Science Teaching Efficacy Belief Instrument (STEBI), an established evaluation tool of science teaching self-efficacy, measured CREP scholars' perceived ability to relay scientific information to lay audiences. The STEBI was administered to CREP students at baseline and post-internship. Participant characteristics and STEBI data were summarized using descriptive statistics, including median and quantile 25th and 75th percentiles for continuous measures and proportions and frequencies for categorical measures from two cohorts. CREP trainees $(n=19)$ self-described their race/ethnicity as Black/African American (68\%), White (26\%), Asian (5\%), Hispanic/Latina (42\%). Trainees were primarily female $(78.9 \%)$. Paired t-test evaluated the STEBI scores difference between pre- and post-training by cohort and for the combined sample. For the combined sample, there were significant differences in scores between pre and post-training STEBI scores $(p=$ 0.002 ) with a pre-training mean score of 31.9 , a post-training mean score of 41.8 , and difference mean score 9.9 respectively. Our results show that trainees demonstrated significantly higher self-efficacy in relaying scientific information to diverse underserved communities. The CREP is an innovative program incorporating community outreach and engagement experiences in which trainees identify the impact of social determinants of health $(\mathrm{SDOH})$ on perpetuating health disparities within underserved communities. Through the CREP, trainees build communication skills and confidence in disseminating useful cancer information and resources to these communities. Objectives: The reviewer shall be able to: Describe the utility of integrating community outreach and engagement experiences in research training programs for underrepresented trainees. Recognize the value in building trainees' self-efficacy in relaying scientific information to underserved communities to address social determinants of health and health disparities. References: Alcaraz, K.I., et al., Understanding and addressing social determinants to advance cancer health equity in the United States: A blueprint for practice, research, and policy. CA: A Cancer Journal for Clinicians, 2020. 70(1): p. 31-46. Alicea-Alvarez, N., et al., Impacting health disparities in urban communities: preparing future healthcare providers for "Neighborhood-Engaged Care" through a community engagement course intervention. Journal of Urban Health, 2016. 93(4): p. 732-743. Gwede, C.K., Bringing a 
Spotlight to the Influences of Social Determinants of Health. Journal of Cancer Education, 2020. 35: p. 211-230.

\section{P41: Correlations between Undergraduate Success and Childhood Experiences}

Cindy Tsai, Lauryn Crudele, France Nguyen-Grozavu, Georgia Robins Sadler

UCSD Moores Cancer Center, La Jolla, CA, USA

Abstract: Research demonstrates that childhood experiences significantly impact adult life. Students' readiness to learn and resilience in college are two outcomes that are impacted by earlier life events. Identifying earlier influential negative/positive childhood experiences and measuring their individual impact may help educators develop innovations to promote personal and college success. The researchers generated a list of potential childhood and teenage factors that were potentially correlated with academic and personal success during college and used them to search the peer-reviewed literature using ERIC, PsycINFO, PubMed, CINAHL, and Google Scholar. Fulltext accessible, English-language articles published between 2015 and 2020 were eligible. Combinations of variations of the following terms were used: childhood, persistence, family, self-determination, peers, expectations, stress, trauma/abuse, food scarcity, shelter insecurity, low income, poverty, college readiness, measurement, psychosocial scales, grit, and motivation. Additional search terms used in the eligible literature were added, along with eligible citations from other eligible articles. Of the 23 factors identified for their potential influence on personal and academic success, the most commonly recognized were family support, financial stability, personal motivation, and grit/resilience. There was an inverse association between college success and on-going family trauma and one-time traumatic events. To explore these relationships further, researchers used survey instruments to quantify the impact of such factors including, completion of self-assessments and rating the factors' impact severity. Having instruments that can make quantitative assessments of these factors makes it possible to rigorously assess programs that provide training and skills acquisition, better financial stability, and enhanced cognitive coping skills. Knowing factors that facilitate students' college success is key to creating effective interventions; tools needed to assess changes in these identified factors are in development. Collaborations among educators can expedite the development of new interventions needed to help at-risk students succeed and the tools needed to rigorously evaluate those interventions. Objectives: The participants shall be able to identify positive and negative associations between early life events and academic success in college. The participants shall be able to recognize the need for interventions that address the specific factors that have an inverse association with college success. The participants shall be able to recognize the need for interventions that build upon the factors that have a direct association with college success. The participants shall be able to recognize the need for qualitative and quantitative assessment instruments that are necessary in the development of evidence-based interventions to promote college success. References: Elphinstone, B., \& Tinker, S. (2017). Use of the Motivation and Engagement Scale-University/ College as a Means of Identifying Student Typologies. Journal of College Student Development 58(3), 457-462. doi:10.1353/ csd.2017.0034. Pike, G.R. Students' Personality Types, Intended Majors, and College Expectations: Further Evidence Concerning Psychological and Sociological Interpretations of Holland's Theory. Res High Educ 47, 801-822 (2006). https://doi.org/10.1007/s11162006-9016-5. St Clair-Thompson, H., Giles, R., McGeown, S. P., Putwain, D., Clough, P., \& Perry, J. (2017). Mental toughness and transitions to high school and to undergraduate study. Educational Psychology, 37(7), 792-809. doi:http://dx.doi.org/10.1080/01443410. 2016.1184746
P42: Factors Influencing Undergraduate Students and Instruments Measuring Student Success in the United States: A Literature Review

Lauryn Crudele, Cindy Tsai, Georgia Robins Sadler, France NguyenGrozavu

UCSD Moores Cancer Center, La Jolla, CA, USA

Abstract: College-level academic science enrichment programs use traditional outcome measures such as graduation rates, employment, starting salaries, and graduate school admissions. This narrative review of the literature searched for alternative measures and possible mediators and moderators of underrepresented students' in a program preparing them for careers addressing cancer disparity. Eligible peer-reviewed literature was identified using ERIC, PubMed, CINAHL, PsycINFO, and Google Scholar and search terms related to undergraduate success in academic and non-academic realms. Eligible articles identified and measured outcomes, mediators, and moderators of academic and personal success among students who were exclusively members of underrepresented communities and interested in STEM, health science, and cancer-related fields. These evaluations were of programs intended to help students successfully transition into college and beyond. Accessible, full-text articles, written in English and published since 2015 were eligible for inclusion. Search terms and reference lists in eligible articles were reviewed to identify additional articles. While multiple undergraduate science enrichment programs were described in the literature, the evaluations predominantly described traditional outcome measures. Among the few nontraditional factors that programs identified as contributing to successful college outcomes were students' ability to establish a long-lasting sense of belonging, establishment of friendships, mental toughness (grit), academic resources and library skills, faculty encouragement, and academic advising. However, the evaluation process for this list of influential factors was not well described and none of the articles reported the use of standardized or non-standardized instruments, nor any efforts underway to validate and norm instruments for assessing these factors. Significant resources are being devoted to creating a more diverse workforce. Focusing on traditional academic outcomes may overlook underrepresented students' barriers to success. Creating a whole-person view of underrepresented students' barriers to academic success, complimented by a holistic approach to preparing them for careers in health and science, warrants exploration. Objectives: The participant shall be able to describe traditional college-level success outcomes and explain why they are incomplete measures of underrepresented students' academic and professional success. The participant shall be able to identify non-traditional college-level success outcomes, mediators, and moderators and explain why they are important to the evaluation of science enrichment education programs for underrepresented college students. The participants shall be able to explain why it is important to evaluate diverse factors impacting underrepresented students' academic and personal success and why validated and normed instruments to do so are critical to develop. References: Johnson SR, Stage FK. Academic Engagement and Student Success: Do High-Impact Practices Mean Higher Graduation Rates? J High Educ [Internet]. 2018 Sep 3 [cited 2020 Jun 25];89(5):753-81. Available from: https://doi.org/10.1080/00221546.2018.1441107. Stock R. Full article: Academic success: the role of mental toughness in predicting and creating success [Internet]. Taylor \& Francis Online. [cited 2020 Jun 25]. Available from: https://www.tandfonline.com/doi/full/10.1080/23752696. 2018.1507623

\section{P43: Utilizing Theory of Planned Behavior to Understand Cancer} Screening Intention among Recreational Sport Participants

Aldenise Ewing ${ }^{1}$, Emily Coughlin ${ }^{2}$, Alicia Best ${ }^{2}$, Joan Dorn ${ }^{3}$, Claudia Parvanta $^{2}$, Bruce Levin ${ }^{4}$, Cathy Meade ${ }^{1}$, Clement Gwede ${ }^{1}$

${ }^{1}$ Moffitt Cancer Center, Tampa, FL, USA, ${ }^{2}$ University of South Florida College of Public Health, Tampa, FL, USA, ${ }^{3}$ The City University of New York, School of Medicine, New York, NY, USA, ${ }^{4}$ University of South Florida, Tampa, FL, USA 
Abstract: Despite being physically active, recreational sport participants (RSPs) are more likely to engage in other behaviors that increase their risk for developing cancer, compared to the general population. Guided by the theory of planned behavior (TPB), the aim of this study was to identify factors associated with cancer screening intention. A cross-sectional, web-based survey including items from the Health Information National Trends Survey (HINTS) was utilized to assess TPB constructs and cancer screening intention among RSPs aged 18-49 years. Descriptive and bivariate analyses were conducted to describe the sample; and multiple linear regression analyses were conducted to assess the impact of demographic variables and TPB constructs on cancer screening intention in this population. Two TPB constructs were significantly associated with intention to screen for cancers including subjective norms (P $<.001)$ and perceived behavioral control $(\mathrm{P}<0$.001). The final analytic model, which included TPB constructs and relevant sociodemographic variables, accounted for nearly $32 \%$ of the variance in intention to screen for cancer. Findings suggest that the TPB could be used to tailor the development of interventions to promote cancer screening among adult RSPs. Consideration should be given to revising attitudinal questions used in HINTS and other national surveys to emphasize positive benefits of prevention rather than lack of individual control. Objectives: The participant shall be able to evaluate the constructs which are related to the intention for cancer screening among recreational sport participants 18-49 using the Theory of Planned Behavior. References: US Bureau of Labor Statistics. Spotlight on Statistics: Participation in Sports and Exercise by Age, 2003-15. National Cancer Institute. Cancer Statistics. 2018; https://www.cancer.gov/about-cancer/understanding/statistics. Accessed September 29, 2019, 2019. Roncancio, A. M., Ward, K. K., Sanchez, I. A., Cano, M. A., Byrd, T. L., Vernon, S. W., FernandezEsquer, M. E., \& Fernandez, M. E. (2015). Using the Theory of Planned Behavior to Understand Cervical Cancer Screening Among Latinas. Health education \& behavior : the official publication of the Society for Public Health Education, 42(5), 621-626. https://doi.org/ $10.1177 / 1090198115571364$

P44: Genetics and Lack of Participation in Clinical Trials Among Hispanic Americans May Negatively Affect Liver Cancer Rates

Naomi Corral, Georgia Robins Sadler, France Nguyen-Grozavu, Christina Jamieson

UCSD Moores Cancer Center, La Jolla, CA, USA

Abstract: Liver cancer (LC) is prevalent among Hispanic Americans (HA). Enough is known about prevention and early intervention strategies to create an education program to help reduce LC mortality among HA. This review of a scientific literature will facilitate the development of a liver cancer education program. To explore whether key elements of HA might correlate with elevated rates of liver cancer, the peer-reviewed scientific literature was explored using PubMed, EBSCO, and Google Scholar. UC San Diego's Roger catalog was also explored for relevant evidence-based information. To be eligible for inclusion, articles needed to be published from 2000-2020 in English or Spanish and accessible in full text. References of eligible articles were examined for other eligible articles. The keywords used included: Hispanics, fatty liver, microbiome, exercise, substance abuse, healthcare access, hepatitis, hepatitis $\mathrm{C}$, and diet. Over a dozen eligible articles were identified. It was found that risk factors of liver disease increase among HA and the efficiency of treatments against them are low. Genetic variation in PNPLA3 is being researched to understand if there is a genetically triggered disparity linked to LC. Hepatitis B can be prevented, while hepatitis C cannot, but effective treatment is available. Risk factors become more prominent when accompanied by a diet high in carbohydrates. HA participation in clinical trials is low, linked to a fear of discrimination, fear of deportation, and language barriers. High LC incidence among HA is due to an increase in liver disease risk factors in this community, attributed to genetics and lack of research on HA and LC. Increased education on clinical trials, treatment cost coverage and treatment options among the HA community may improve LC rates. Objectives: The participants shall be able to identify at least three causes of liver cancer disparities among Hispanic Americans. References: Wagenknecht, L. E., Palmer, N. D., Bowden, D. W., Rotter, J. I., Norris, J. M., Ziegler, J., Chen, Y.-D. I., Haffner, S., Scherzinger, A., \& Langefeld, C. D. (2011). Association of PNPLA3 with non-alcoholic fatty liver disease in a minority cohort: The Insulin Resistance Atherosclerosis Family Study. Setiawan, V. W., Wei, P. C., Hernandez, B. Y., Lu, S. C., Monroe, K. R., Marchand, L. L., \& Yuan, J. M. (2016). Disparity in liver cancer incidence and chronic liver disease mortality by nativity in Hispanics: The Multiethnic Cohort. Cancer, 122(9), 1444-1452.

\section{P45: Using an Ecological Model and FIT to Increase Colorectal} Cancer Screening Rates in an Urban Community

Luis Alzate-Duque ${ }^{1}$, Ashna Shome ${ }^{1}$, Pamela Valera ${ }^{2}$, Ana NatalePereira $^{1}$, Vivian Cueto ${ }^{1}$

${ }^{1}$ Rutgers New Jersey Medical School, Newark, NJ, USA, ${ }^{2}$ Rutgers, State University of New Jersey, Newark, NJ, USA

Abstract: Colorectal cancer screening reduces morbidity and mortality. In Essex County, $\mathrm{NJ}$ age-adjusted prevalence for completion of screening was $71.5 \%$ in 2017. Black and Hispanic/Latinx residents had decreased screening rates $(65.3 \%)$. This project implements an ecological model to increase screening rates in urban populations using community engagement, education, navigation and FIT. Study recruitment occurred at community-based events following a colorectal cancer education session. During the education session, community members received education on CRC risks and screening. These sessions were carried out in English, Spanish and Portuguese. Eligibility criteria included being between the ages of 45-85 and not being up-to-date with USPSTF recommended colorectal cancer screening. Eligible participants who agree to participate were provided a FIT kit and instructed on completion of FIT kits to be returned within 2 weeks. Patient navigators performed language concordant follow-up to address barriers to completion and facilitate timely FIT return. In total, 132 persons received a FIT kit. Fifteen participants $(11 \%)$ were male. Mean age of those receiving FIT kits was 60.6 years. $71 \%$ of persons reported no primary care provider (PCP), $72 \%$ reported no insurance, and $81 \%$ reported no prior CRC screening. Language preference included Spanish, Portuguese and English. Of 73 FIT kits returned, 9 (12\%) were positive for occult blood, and 2 diagnostic colonoscopies have been completed. Barriers to completion include misplaced or lost FIT kit, lack of time, and feeling uncomfortable handling fecal sample. We found no association between PCP status, insurance status, and prior screening. Our model can be used to target and improve colorectal screening rates for underserved populations. Our project creates opportunity for future research, especially FIT kit uptake/return, barriers to screening, with the opportunity to expand among marginalized populations such as individuals affected by the justice system, LEP individuals, and minority communities. Objectives: The participant shall be able to identify at least one interpersonal, intrapersonal, and community based interventions within this project as it pertains to improving colorectal cancer screening. The participant shall be able to identify at least 1 barrier to the completion of CRC screening via FIT. The participant shall be able describe a multidisciplinary approach to CRC screening for underserved populations. References: Paul G. Devereux, PhD, MPH, John Gray, MD, Susan Robinson, MBA, Janie Galvin, BS, and Jesse Gutierrez, MS. Using Community Engagement and Navigators to Increase Colon Cancer Screening and Patient Outcomes. Health Promotion Practice, February 9, 2018 https://doi.org/10.1177\%2F1524839918757485. Selby K, Baumgartner C, Levin TR, Doubeni CA, Zauber AG, Schottinger $\mathrm{J}$, et al. Interventions to Improve Follow-up of Positive Results on Fecal Blood Tests: A Systematic Review. Ann Intern Med. 2017;167:565-575. doi: 10.7326/M17-1361 
P46: A multilevel exploration of determinants of human papillomavirus vaccine initiation among U.S. Vietnamese

\author{
Milkie $\mathrm{Vu}^{1}$, Carla Berg ${ }^{2}$, Cam Escoffery ${ }^{1}$, Victoria Huynh ${ }^{1}$, Robert \\ Bednarczyk $^{1}$ \\ ${ }^{1}$ Emory University, Atlanta, GA, USA, ${ }^{2}$ George Washington University, \\ Washington, DC, USA
}

Abstract: U.S. Vietnamese experience higher cervical cancer incidence and have lower human papillomavirus vaccine (HPVV) uptake compared to other racial/ethnic groups. Limited research has examined multilevel determinants of HPVV uptake among this population. We used the P3 (practice-, provider-, and patient-level) model [1] to examine multilevel determinants of U.S. Vietnamese. In 2020, we conducted an online crosssectional survey of Vietnamese parents living in the U.S. who had $\geq$ one child aged 9-18. Parents were recruited through U.S. Vietnamese community-based organizations, U.S. Vietnamese-focused Facebook groups and listserv, and snowball sampling. Descriptive statistics and Chi-square tests were conducted to understand facilitators of and barriers to HPVV initiation (i.e. 1+ vaccine dose). The sample represented 331 Vietnamese parents in 35 states. Most were female $(84.6 \%)$, had $\geq$ Bachelor's degree (87.3\%), and income of $\$ 50,000+(65.6 \%) ; 98.8 \%$ were born outside of the U.S. Less than half of parents initiated HPVV for their children (43.5\%). HPVV initiation significantly differed by whether parents had seen HPVV materials at their children's clinics ( $62.3 \%$ vs $26.2 \%, \mathrm{p}<.001)$. The most common facilitator of adolescent HPVV initiation was a doctor's recommendation (80.0\%). HPVV initiation significantly differed by child's sex $(52.4 \%$ for female adolescents vs $33.3 \%$ for males, $\mathrm{p}<.001)$. Almost all parents $(97 \%)$ believed adolescent vaccines are safe. In this sample, adolescent HPVV initiation (43.5\%) was much lower than general U.S. populations (68.1\% in 2018 NISTeen data) [2]. Some findings were well-identified barriers (e.g., doctor's recommendation, child's sex) while others were novel (e.g., clinic-based materials about HPVV). Interventions can incorporate findings regarding high degree of trust in vaccines. Objectives: Compare HPVV initiation prevalence among U.S. Vietnamese to general U.S. populations. Identify multilevel facilitators of and barriers to HPVV initiation among U.S. Vietnamese References: [1] Bednarczyk RA, Chamberlain A, Mathewson K, Salmon DA, Omer SB. Practice-, Provider-, and Patientlevel interventions to improve preventive care: Development of the P3 Model. Prev Med Reports 2018;11:131-8. doi:10.1016/ j.pmedr.2018.06.009. [2] Walker TY, Elam-Evans LD, Yankey D, Markowitz LE, Williams CL, Fredua B, et al. National, Regional, State, and Selected Local Area Vaccination Coverage Among Adolescents Aged 13-17 Years - United States, 2018. MMWR Morb Mortal Wkly Rep 2019;68:718-23. doi:10.15585/mmwr.mm6833a2.

\section{P47: Testing the Effectiveness of Oral and Written Breast Cancer} Education Methods among Amish and Mennonite Women

\section{Melissa Thomas ${ }^{1}$, Doretta Thomas ${ }^{2}$ \\ ${ }^{1}$ Ohio University Heritage College of Osteopathic Medicine, Athens, $\mathrm{OH}$, USA, ${ }^{2}$ Center for Appalachia Research in Cancer Education, Newport, $\mathrm{OH}, \mathrm{USA}$}

\begin{abstract}
Ohio is home to the world's largest and fourth largest Amish settlements with disproportionately higher breast cancer mortality rates. The purpose of this study was to test the effectiveness of a culturally competent breast cancer education program that included a follow-up educational flyer mailed to participants at a rural screening. Amish and Mennonite Women participating in a screening day completed a pretest survey of key breast cancer knowledge questions. New participants received the standard, full-length education. Old participants who participated at least once in the past two years receive a tailored education intervention on the specific questions answered incorrectly. A randomized group of half of the participants received a mailing three months after
\end{abstract}

the education session that included the key breast health messages shared in the education program. All participants received a post-test of the same knowledge questions six months after the intervention. The mean age was 55.2 years. A total 163 posttests were returned between October 2017 February 2019 with a $50.15 \%$ response rate. There was a significant increase between pre- and post-test scores for both old and new education interventions at six months $(\mathrm{p}=.0005)$. However, receipt of the educational flyer had no impact on post-test scores. Regardless of the education received, there was no significant difference in knowledge levels between those who received the flyer and those who did not, even when controlling for age. $(\mathrm{p}=.551)$. One-on-one oral breast cancer education programs can be effective in Amish and Mennonite communities, but the use of a follow-up written educational flyer may not be effective for multi-literate population groups when English is a second language. Additional research is warranted on specific breast cancer beliefs and knowledge over time. Objectives: The participant shall be able to list two breast cancer knowledge facts that did not improve with the education intervention among Amish and Mennonite women who participated in a women's health screening program. The participant shall be able to identify the impact of an educational flyer in increasing breast cancer knoweldge among Amish and Mennonite women who participate in a women's health screening program. References: Geiger, SD \& GrigsbyToussaint, DJ. (2017). Mammography seeking practices of Central Illinois Amish women. Journal of Community Health, 42(2), 369-376. https://doi.org/10.1007/s10900-016-0265-8. McBridge, K., \& Gesink, D. (2018). Increasing cancer screening among Old Order Anabaptist women through specialized women's health and integrated cancer screening interventions. Journal of Immigrant and Minority Health, 20(2), 465-578.

P48: African-American Participation in Oncologic Clinical Trials: A Review of Barriers and Potential Solutions

\author{
Quinton Blount ${ }^{1}$, Benjamin Wibonele ${ }^{2}$, Charles Moore ${ }^{2}$ \\ ${ }^{I}$ Emory University School of Medicine, Columbus, GA, USA, ${ }^{2}$ Emory \\ University Department of Otolaryngology, Atlanta, GA, USA
}

Abstract: Diverse and inclusive clinical trial results benefit broad populations. African-Americans have disproportionately low participation in trials due to barriers, though strategies are available to overcome them. Our study outlines socioeconomic and medical implications of these barriers to expose inequities and eradicate difficulties African-Americans face prior to enrollment. We conducted a systematic rapid review of factors influencing African-American participation in cancer clinical trials. Our PubMed search generated 622 articles published this decade. We then refined our search to fit our exclusion/inclusion criteria by only incorporating articles generated from a search including oncology, clinical trial, participation, barrier and African American. This search produced 86 articles for review. From these, we identified barriers to clinical trials, etiology, and mechanisms to overcoming them. Many social determinants relate to wealth attainment or lack thereof. Findings as listed; communication, willingness to participate, trial eligibility, community awareness, comorbidity prevalence, SES, insurance, transportation, and trial compensation are directly or indirectly related to wealth. Barriers are influenced by systematic racism and social determinants of health. Socioeconomic factors influence logistic factors influencing willingness to participate. Communication is key linguistically and culturally, as is willingness to participate despite medical mistrust and negative attitudes towards research due to historical experimentation and exploitation of African-Americans. Comorbidity prevalence in African-Americans, influenced by socioeconomic status and access to healthcare, elucidate these barriers. Physicians should address biases toward African Americans to promote trial opportunities. Community-based approaches like leveraging churches and nonprofits improve awareness. Community programming mitigates medical mistrust. Patient navigator intervention coordinates care. Proposed strategies must consider and address newly presenting barriers. Objectives: 1 . The participant shall be able to identify 
key barriers to oncologic clinical trials in the African American community. 2. The participant shall be able to discuss proposed strategies for overcoming barriers to oncologic clinical trials. References: 1 . Sprague Martinez L et al. Clinical Trials in the Black Community: Implications for Care Coordination. 2. Ahaghotu $\mathrm{C}$ et al. African American Participation in Oncology Clinical Trials-Focus on Prostate Cancer: Implications, Barriers, and Potential Solutions. 3. Pelto DJ et al. Adaptation of a Cancer Clinical Trials Education Program for African American and Latina/o Community Members. 4. Green MA et al. Evaluating a community-partnered cancer clinical trials pilot intervention with African American communities. 5. Rivers D et al. A systematic review of the factors influencing African Americans' participation in cancer clinical trials. 6. Ghebre RG et al. State-of-the-science of patient navigation as a strategy for enhancing minority clinicaltrial accrual.

\section{P49: Difficulties Faced by Researchers in Pancreatic Cancer Clinical Trial Recruitment Within the African American Community}

Shawn Barrowcliff ${ }^{1}$, Georgia Robins Sadler ${ }^{2}$, Scott Kelley ${ }^{1}$, France Nguyen-Grozavu ${ }^{2}$

${ }^{1}$ San Diego State University, San Diego, CA, USA, ${ }^{2}$ UCSD Moores Cancer Center, La Jolla, CA, USA

Abstract: African Americans (AA) suffer disproportionately high burdens of pancreatic cancer, a cancer without early symptoms, early diagnostic options, or effective late-stage treatment options, and as a consequence, they suffer a high mortality rate. Discovering non-invasive screening methods will be of limited value if AA are underrepresented in these clinical trials. Scientists need recruitment strategies as innovative as their scientific ideas and should focus on communities that will benefit most from their discoveries. This literature review attempted to identify AAs' barriers to clinical trial participation, as well as socio-culturally aligned ways to help AA evaluate whether participation in particular clinical trials are appropriate. Articles published since 2015 were found in PubMed, CINAHL, and Google Scholar using combinations of such terms as cancer, pancreas*, AA, clinical trial, Black, recruit*, and distrust. Reference lists of articles were searched for additional articles. American Cancer Society and National Cancer Institute pancreatic cancer statistics were used. Over forty articles were used to identify key tasks. First, researchers must know where to find eligible participants, within the community and/or the medical system. Then socio-culturally aligned approaches are needed for disseminating study information, snowball contacting methods, outreach at community venues, one-on-one conversations, and meaningful relationships with community leaders. Researchers must also communicate all expectations of study participants and be aware that AA vividly recall repeated examples of unethical research practices that produced distrust, fear, and cultural opposition to clinical trials. Such stellar outreach education/recruitment programs require adequate staff and participant incentives, underscoring the need for adequate budget planning. Increasing AAs' representation in clinical trials begins with a well-developed, culturally aligned recruitment plan, accompanied by a well-justified budget that enables researchers to implement culturally competent education and recruitment strategies. Encouraging study participants to share their positive clinical trial experiences could also help increase community-wide interest in clinical trials participation. Objectives: The participant shall be able to recognize the importance of having African American participation in research studies for pancreatic cancer. The participant shall also be able to identify strategies and possible techniques to recruit African Americans into clinical trials. References: Hughes TB, Varma VR, Pettigrew C, Albert MS (2017) African Americans and Clinical Research: Evidence Concerning Barriers and Facilitators to Participation and Recruitment Recommendations. Gerontologist 57:348-358. https://doi.org/10.1093/geront/gnv118. Otado J, Kwagyan J, Edwards D, et al (2015) Culturally Competent Strategies for Recruitment and Retention of African American Populations into Clinical Trials. Clin Transl Sci 8:460-466. https://doi.org/10.1111/cts. 12285
P50: Cancer Screening Educational Interventions in Rural Farmworker Communities: A Systematic Literature Review

Laura Garcia-Torres ${ }^{1}$, Caitlyn Johnson ${ }^{1}$, Anni Liu ${ }^{1}$, Shannon M. Christy $^{2}$, Clement Gwede ${ }^{2}$

${ }^{1}$ University of South Florida Morsani College of Medicine, Riverview, FL, USA, ${ }^{2}$ Moffitt Cancer Center, Tampa, FL, USA

Abstract: There are approximately 3 million farmworkers in the United States (U.S.), of whom $83 \%$ identify as Hispanic/Latino. Multiple social determinants of health result in barriers to cancer screenings and preventive medicine for farmworker communities. This systematic literature review examined educational interventions aimed at increasing cancer screening behaviors in this population. A protocol was used to search articles published between 2002-2021 in the following databases: Ovid MEDLINE, CINAHL Complete, Embase, and Web of Science. Search terms included: "agricultural," "rural," "cancer prevention," and "screening." Studies were included if they reported screening adherence, were conducted in the U.S., published in English, and involved cancer education in the intervention. We then identified interventions designed to increase cancer screening adherence among rural farmworkers (with participants of any age, gender, or ethnicity). Preferred Reporting Items for Systematic Reviews and Meta-Analyses guidelines were followed. Two independent reviewers screened 703 articles; 136 articles met criteria for full-text screening and 26 articles were eligible for data extraction. Due to the heterogeneity of these articles, we focused on the six interventions targeted to farmworker communities. Two studies were randomized and three focused only on women. Common intervention strategies included reducing structural barriers and use of culturally-relevant educational materials. Interventions led by lay health educators showed the greatest efficacy with a significant increase in cancer screening behaviors. The randomized studies showed an increase in adherence ranging from 22.3\%-53.4\%, while non-randomized showed an increase of adherence ranging from $10 \%-33 \%$. Culturally-relevant interventions were effective in increasing cancer screening behaviors among those living in rural farmworker communities. Lay health educator-led educational interventions demonstrated especially promising results. Further research in the development and piloting of male-focused interventions is suggested to address current gaps in this community. Objectives: 1 . The participant shall be able to identify at least three concurrent barriers which rural farmworker communities face with access to quality cancer prevention and screenings. 2. The participant shall be able to define Promotoras and Charlas and their role in increasing adherence to cancer education. 3.The participant shall be able to identify future directions needed in order to fill current gaps within the farmworker community. References: Fleming K, Simmons VN, Christy SM, et al. Educating Hispanic Women about Cervical Cancer Prevention: Feasibility of a Promotora-Led Charla Intervention in a Farmworker Community. Ethn Dis. 2018;28(3):169176. Published 2018 Jul 12. doi:10.18865/ed.28.3.169. Furgurson KF, Sandberg JC, Hsu FC, Mora DC, Quandt SA, Arcury TA. Cancer Knowledge Among Mexican Immigrant Farmworkers in North Carolina. J Immigr Minor Health. 2019;21(3):515-521. doi:10.1007/ s10903-018-0771-0

P51: Facilitators and barriers to HPV vaccination among Young Latino Men who have Sex with Men: A systematic review

Melissa Marzán-Rodríguez ${ }^{1}$, Shannon M. Christy $^{2}$, Lorena Brito ${ }^{1}$, Cheryl Gonzalez ${ }^{1}$, Anthony Perez ${ }^{1}$, Cristina Perez ${ }^{1}$, Julio Jimenez, Susan Vandaparampil ${ }^{2}$

${ }^{1}$ Ponce Health Sciences University, Ponce, PR, USA, ${ }^{2}$ Moffitt Cancer Center, Tampa, FL, USA

Abstract: Human papillomavirus (HPV) is the most common sexually transmitted infection in United States (U.S.). Men who have Sex with Men (MSM) are at increased risk for acquiring an HPV infection and 
subsequently developing an HPV-related cancer. Despite their increased risk for HPV infection, only $13.7 \%$ of MSM report receiving the HPV vaccine. To understand the state of the science of HPV vaccine behavioral literature focused on young Latino MSM (YLMSM), we conducted a systematic review guided by PRISMA using PubMed and EBSCO databases. All articles were reviewed focusing on knowledge, awareness, attitudes, and risk perceptions of HPV vaccination in the YLMSM population ( $<29$ years of age) in U.S. and Puerto Rico and were published between January 2010-June 2020. A total of 64 articles were identified of which 9 were duplicates. Thirty-five articles were excluded in the screening process. A total of $n=18$ articles met criteria and were included for the systematic review analysis. Educational interventions focused on YLMSM and HPV vaccination were scarce, and were limited to English speakers in the U.S. Barriers to HPV vaccination for YLMSM identified were: lack of HPV vaccine knowledge, lack of access to healthcare providers willing to provide HPV vaccine, and stigma related to sexual orientation. Educational interventions are urgently needed to increase HPV vaccine knowledge and uptake among YLMSM. Specifically, Spanish language interventions are needed for Spanishpreferring YLMSM. Increasing HPV vaccine uptake among YLMSM can reduce HPV-related cancer and health disparities in the future. Objectives: The participants should be able to identify, at least, four barriers related to HPV awareness and HPV vaccine uptake among high risk group: Young Latino Men who have Sex with Men (YLMSM). References: Kellogg C; et al. 2019. Fontenot HB; et al. 2019. Feeney L; et al. 2019. Koskan AM; et al. 2018. Cooper DL; et al. 2018. Gorbach PM; et al. 2017. Cooper DL; et al. 2017. Fontenot HB; et al. 2016. Dionne P; et al. 2016. Fenkl, E.A.; et al. 2016. Cummings T; et al. 2015. Reiter PL; et al. 2015

P52: Increasing Breast and Cervical Cancer Screening Interventions in Rural Communities: A Systematic Literature Review

Caitlyn Johnson ${ }^{1}$, Laura Garcia-Torres ${ }^{1}$, Anni Liu ${ }^{1}$, Mary Katherine Haver $^{2}$, Clement Gwede ${ }^{2}$, Shannon M. Christy ${ }^{2}$

${ }^{1}$ University of South Florida Morsani College of Medicine, Riverview, FL, USA, ${ }^{2}$ Moffitt Cancer Center, Tampa, FL, USA

\begin{abstract}
Despite established breast and cervical cancer screening recommendations, rural Americans are less likely than non-rural to be screened which contributes to diagnostic delays, later stage at diagnosis, and higher mortality. This systematic review examines educational interventions aimed at increasing breast and cervical cancer screening in rural areas of the U.S. CINAHL Complete, Ovid MEDLINE, Embase, and Web of Science databases were searched using multiple terms (e.g., "rural", "agriculture", "cancer screening", "prevention"). Eligible studies met the following criteria: rural or agricultural U.S. population, had cancer screening adherence as an outcome variable, and publication date of 2002-2021. Resulting studies were categorized as farmworker communities and rural communities. PRISMA guidelines were utilized to perform data abstraction and coding. Methodological quality was assessed per Cochrane guidelines. For the current study, our focus was educational interventions targeting breast and cervical cancer screening in rural women. Twenty articles met "rural" inclusion criteria; ten targeted breast and/ or cervical cancer screening outcomes. All ten evaluated patient-level interventions. Two were randomized controlled trials (RCTs) and eight were non-randomized. The eight non-RCTs utilized pre/post-test, two-, or three-arm quasi-experimental designs. Eight of the ten studies reported statistically significant screening increases from baseline ( $p$-values ranged from $<0.0001$ to 0.05 ). Use of lay health advisors (LHA) demonstrated post-intervention screening increases of $6 \%-65.8 \%$. In the single study utilizing message framing, an $87 \%$ increase in mammography was reported. Effective educational intervention strategies included LHAs, targeted education/messaging, and financial subsidies to offset the cost of screenings. Results demonstrate the value of LHAs and targeted education to increase breast and cervical cancer screening in rural communities. These
\end{abstract}

intervention strategies successfully increased screening and should be considered essential in future studies. Prospective research should explore use of financial partnerships and provider-level or organizational-level interventions to increase overall impact. Objectives: The participant shall be able to define "lay health advisors" and identify their utility in increasing breast and cervical cancer screening and prevention. The participant shall be able to identify common components of efficacious educational interventions in a rural setting. The participant shall be able to identify gaps in literature pertaining to breast and cervical cancer screening and prevention in rural settings. References: Chandak A, Nayar P, Lin G. Rural-Urban Disparities in Access to Breast Cancer Screening: A Spatial Clustering Analysis. J Rural Health. 2019;35(2):229-235. doi:10.1111/ jrh.12308. Falk D. A Mixed Methods Review of Education and Patient Navigation Interventions to Increase Breast and Cervical Cancer Screening for Rural Women. Soc Work Public Health. 2018;33(3):173186. doi: $10.1080 / 19371918.2018 .1434583$

\section{P53: Educational Intervention Studies Aimed at Increasing Colorectal Cancer Screening Among Men and Women in Rural Communities: A Systematic Literature Review}

Anni Liu ${ }^{1}$, Laura Garcia-Torres ${ }^{1}$, Caitlyn Johnson ${ }^{1}$, Mary Katherine Haver $^{2}$, Clement Gwede ${ }^{2}$, Shannon M. Christy ${ }^{2}$

${ }^{1}$ University of South Florida Morsani College of Medicine, Riverview, FL, USA, ${ }^{2}$ Moffitt Cancer Center, Tampa, FL, USA

Abstract: Rural communities experience higher cancer incidence and mortality compared to non-rural communities due to multiple factors including social determinants of health. The goal of this systematic literature review was to examine the landscape of educational interventions designed to increase colorectal cancer (CRC) screening among rural communities in the United States. Using the Preferred Reporting Items for Systematic Reviews and Meta-Analyses (PRISMA) guidelines, our search employed the following databases: Ovid MEDLINE, CINAHL Complete, Embase, and Web of Science. Search terms included, but were not limited to: "rural," "cancer screening," and "cancer prevention." Eligible studies were published in English, between 2002-2021, implemented in agricultural or rural United States settings, involved male and/ or female participants, and featured an educational intervention with a cancer screening behavioral outcome. Eligible articles were then narrowed in scope to those targeting CRC screening. Article screening and data extraction were conducted by two independent reviewers. Eight articles targeted CRC screening in rural populations. Three studies were randomized-controlled trials (RCT) and five were non-RCT. Intervention strategies ranged from standard mailed CRC educational materials to tailored community-based educational sessions. Three studies reported statistically significant differences in CRC screening rates in intervention arms compared to control arms (range:17-39.4\%) or increase from baseline (range: $36-40 \%$ ). Half of these studies focused on screening with a stool blood test (SBT) explicitly, while the remainder measured either a combination or any CRC screening test. The most efficacious intervention strategies included educational materials mailed along with SBT and community-based educational presentations. Findings indicate that cancer education and access to testing play an important role in promoting CRC screening in rural communities. Future research should explore ways to raise uptake rates to the national $80 \%$ goal and improve repeat/ annual SBT screening adherence, and the impact of health system-level interventions needs further exploration. Objectives: 1 . The participant shall be able to identify the utility of "stool blood test" in colorectal cancer screening. 2. The participant shall be able to identify two efficacious interventions that led to improvements in colorectal cancer screening. 3. The participants shall be able to identify future directions needed to close the gap in colorectal cancer screening in rural communities. References: Arnold CL, Rademaker AW, Morris JD, Ferguson LA, Wiltz G, Davis TC. Follow-up approaches to a health literacy intervention to increase colorectal cancer screening in rural community clinics: A randomized 
controlled trial. Cancer. 2019;125(20):3615-3622. doi:10.1002/ cncr.32398. Preston MA, Glover-Collins K, Ross L, et al. Colorectal cancer screening in rural and poor-resourced communities. Am J Surg. 2018;216(2):245-250. doi:10.1016/j.amjsurg.2017.08.004

\section{P54: The disparity of cancer treatment for $\mathrm{HIV}+$ patients and the importance of HIV specific clinical trials}

Rowan Ustoy, Georgia Robins Sadler, France Nguyen-Grozavu UCSD Moores Cancer Center, La Jolla, CA, USA

\begin{abstract}
Anti-retroviral therapy extended the life of people with HIV and decreased the risk of AIDS. Although the number of AIDS-defining cancers (ADC) has decreased, the number of non-ADC increased, making it helpful to know what advances are being reported that could improve the future care of people with HIV/AIDS and cancer. This narrative literature review focused on identifying the latest advances in cancer treatment for patients with HIV and any disparities in their cancer outcomes. Full-text articles published in English since 2000 were eligible. The following search terms were used in combination: HIV, cancer, treatment, disparities, and clinical trials. The terms were entered into PubMed, Google Scholar, and CINAHL. All articles referred specifically to the United States HIV population. Twelve key articles were found as well as four key websites: The AIDS Malignancy Consortium (AMC), National Institute of Health/ National Cancer Institute (NIH/NCI), Patient Navigator Training Collaborative (PNTC), and Women's Interagency HIV Study (WIHS). Patients with cancer and HIV are often diagnosed in later stages and receive cancer treatment later or not at all, contributing to higher mortality rates. This is especially true for African Americans and Latinx. These disparities are thought to be a mixture of patient, physician, health systems, and socioeconomic barriers. Patients struggle with the long-term burden of treatments. Physicians do not receive sufficient education regarding the optimal care of patients with HIV and cancer. Clinical trials previously excluded participants with HIV and they remain underrepresented. Programs, like the AMC and WIHS, are currently focused on decreasing these disparities by conducting HIV-inclusive clinical trials. Increasing the representation of people with HIV in HIV-specific and non-specific cancer clinical trials will expedite the development of effective cancer treatments. Training programs, like PNTC, and easy access to consultation with an HIV specialist should be available to oncologists. This would help oncologists to treat patients with HIV and cancer using the latest discoveries. Objectives: The participant shall be able to identify at least two barriers that HIV+ cancer patients face to receive proper access to care. The participant shall be able to identify at least one way to improve treatment for HIV+ cancer patients. The participant shall be able to identify one online resource that works to decrease the disparity of cancer treatment for HIV+ patients. References: Coghill, Anna, and Gita Suneja. "Cancer Care Disparities in People with HIV in the United States." Current Opinion in HIV and AIDS, vol. 12, no. 1, 2017, pp. 63-68., doi:10.1097/coh.0000000000000320. Little, Richard F. "Cancer Clinical Trials in Persons with HIV Infection." Current Opinion in HIV and AIDS, U.S. National Library of Medicine, Jan. 2017, www.ncbi.nlm.nih.gov/pubmed/27559711. Suneja, Gita, et al. "Disparities in Cancer Treatment among Patients Infected with the Human Immunodeficiency Virus." American Cancer Society Journals, John Wiley \& Sons, Ltd, 17 May 2016, acsjournals.onlinelibrary.wiley.com/doi/abs/ $10.1002 /$ cncr.30052.
\end{abstract}

\section{P55: Genetic Screening for Cancer Risk and Cancer Disparities}

Richard Burnett, Georgia Robins Sadler

UCSD Moores Cancer Center, La Jolla, CA, USA

Abstract: The discovery of next-gen sequencing technologies has made genetic counseling an essential tool for preventative medicine. While in 2010 it would cost an individual $\$ 50,000$ to sequence their genome, currently, a whole genome can be sequenced for under $\$ 1,000$ and can be covered by most insurances. This drastic change in availability over time raises question of its potential inequitable distribution. Are genetic testing disparities directly contributing to cancer disparities? A literature review was conducted using the databases pubmed and CINAHL using the keywords: Genetic counseling, genetic testing, genetic screening, disparity, and cancer. Narrowed down to 50 articles all published between 2010 and 2020 that seemed relevant and with further review, 26 articles were selected. Only data from the United States was included and any other regions were excluded. Genetic testing allows for preventative measures to be used to greatly improve cancer outcomes and improves incidence rates; however certain populations disproportionately benefit from this tool. African Americans with a family history of cancer were less likely to use genetic testing for cancer risk than their white counterparts. It is still a financial barrier to very low-income individuals, and especially to those who are uninsured. Low awareness and knowledge of genetic testing for and counseling played a role in determining uptake amongst ethnic minority and racial groups within the United States. Literature is abundant for genetic testing disparities within ethnic minorities but there is a dearth in exploration among other underrepresented groups. Further research is needed to establish a direct link between genetic screening and cancer disparities, however it is likely that both are linked to similar underlying health inequities. Objectives: The participant shall be able to identify a barrier to genetic screening for cancer risk. References: Hann KEJ, Freeman M, Fraser L, Waller J, Sanderson SC, Rahman B, et al. Awareness, knowledge, perceptions, and attitudes towards genetic testing for cancer risk among ethnic minority groups: a systematic review. BMC Public Health. 2017 25;17(1):503. Kurian AW, Ward KC, Howlader N, Deapen D, Hamilton AS, Mariotto A, et al. Genetic Testing and Results in a Population-Based Cohort of Breast Cancer Patients and Ovarian Cancer Patients. J Clin Oncol. 2019 20;37(15):1305-15.

P56: Efficacy and quality of cancer research training provided by the ReTOOL program for its $\mathbf{2 0 2 0}$ cohort of underrepresented minority students

Parisa Fathi ${ }^{1}$, Folakemi Odedina ${ }^{1}$, Bereket Mochona ${ }^{2}$, Renee Reams ${ }^{2}$, Jennifer Nguyen ${ }^{3}$, Nissa Askins ${ }^{1}$, Ernest Kaninjing ${ }^{4}$, Linda BeharHorenstein ${ }^{1}$, Debra Lyon ${ }^{1}$, Merry Jennifer Markham ${ }^{1}$

${ }^{1}$ University of Florida, Orlando, FL, USA, ${ }^{2}$ Florida A\&M University, Tallahassee, FL, USA, ${ }^{3}$ Mercer University, Macon, GA, USA, ${ }^{4}$ Georgia College \& State University, Milledgeville, GA, USA

Abstract: The curriculum of the Research Training Opportunities for Outstanding Leaders (ReTOOL) Program at the University of Florida (UF) includes lab, discussion, and lectured-based training about cancer research and health disparities. The 2020 ReTOOL cohort included 17 underrepresented minority (URM) undergraduate students -5 Hispanic/ Latinx and 12 Black/African American - and is the 8th cohort of the program. Students completed pre- and post-program surveys to provide programmatic evaluation, primarily the program's ability to teach cancer research skills and understanding of relevant concepts. The pre- and postprogram surveys asked the students to reflect on their understanding of research, developing research, writing grants, writing proposals, averting scientific misconduct, and understanding the role of collaboration through 6 short-answer responses at the time of survey completion. Responses were compared and contrasted to determine if increases or improvements, decreases, or no changes in skills and knowledge occurred individually and cohort-wide. If the cohort increased skills or knowledge in any area, then the curriculum was effective. Overall, the 2020 ReTOOL students noticed an increase or an improvement in skills and knowledge in 5 of the 6 areas compared to when they began ReTOOL. Understanding research practices was the most improved area with 15 of 17 students $(88 \%)$ reporting change. Students attributed their improvement to the support and feedback from their assigned lab, the speakers' topics during Didactic Orientation, and required trainings. Grant writing 
was the least improved area with only 2 of the 17 students (18\%) noting improvement; several students reported little to no exposure to grant writing throughout the program. No decreases were reported. The 2020 ReTOOL students are prepared to continue cancer research dafter completing the program. The different methods of content delivery and strong support from others greatly attribute to an effective cancer research training program for URM undergraduate students. Training URM students will lead to more URM researchers reducing macro and micro level cancer health disparities. Objectives: The participant shall be able to complete a pre-program survey and a post-program survey. The participant shall be able to reflect upon their understanding of and experience with 6 areas of research (research practices, developing a research process, writing grants, writing proposals, averting scientific misconduct, role of collaboration). The participant shall be able to write responses to the 6 short-answer questions about the areas of research. The participant shall be able to respond to the questions based on their understanding of and experience with the 6 areas of research at the time of survey completion. References: Odedina, F.T., Reams, R.R., Kaninjing, E. et al. Increasing the Representation of Minority Students in the Biomedical Workforce: the ReTOOL Program. J Canc Educ 34, 577-583 (2019). https://doi.org/10.1007/s13187-018-1344-6. Fisher AJ, MendozaDenton R, Patt C, et al. Structure and belonging: Pathways to success for underrepresented minority and women $\mathrm{PhD}$ students in STEM fields. PLoS One. 2019;14(1):e0209279. Published 2019 Jan 9. doi:10.1371/ journal.pone.0209279

P57: HPV-associated anal cancer knowledge, attitudes, and health communication among non-clinical staff at HIV/AIDS service organizations in Southern United States region.

Aditi Tomar $^{1}$, Olufunto Olusanya ${ }^{2}$, Lisa Tisdale Wigfall ${ }^{1}$

${ }^{1}$ Texas A\&M University, College Station, TX, USA, ${ }^{2}$ University of Tennessee Health Science Center - Oak Ridge National Laboratory (UTHSC-ORNL) Center for Biomedical Informatics, Memphis, TN, USA

Abstract: Co-infection with HIV/HPV and bio-behavioral risk factors (e.g. immunodeficiency, un-protected sex etc.) increase likelihood for developing anal and other HPV-associated cancers among people living with HIV/AIDS (PLWH). We explored knowledge, attitudes, and health communication regarding HPV-associated anal cancer among HIV/AIDS service organizations (ASO) non-clinical employees who provided services to PLWH. Employees $(n=59)$ were recruited from six ASO locations in the Southern United States. Participants completed a structured, self-administered needs assessment survey. For the current investigation, variables were limited to knowledge, attitudes, and health communication regarding HPV-related anal cancer. Descriptive statistics including frequencies and percentages analyzed knowledge, attitudes, and health communication among ASO staff. Study measures were dichotomized into binary variables i.e. "high" or "low" knowledge, attitude, and health communication. Fisher's exact test analyzed for statistical differences in knowledge, attitudes, and health communication among ASO staff grouped by sex/gender into heterosexual females, heterosexual males, LGBTQ+ females, and LGBTQ+ males. ASO employees $(n=59)$ were mean age $45.5 \pm 13.5 \mathrm{SD}$, range $22-70$ years. Participants were heterosexual females $(47 \%)$, heterosexual males (14\%), LGBTQ+ females $(14 \%)$, LGBTQ+ males $(25 \%)$. Mean scores were: knowledge $(1.7 \pm$ $1.35 \mathrm{SD}$, range $0-4)$, attitude $(2.2 \pm 0.67 \mathrm{SD}$, range $0-3)$, and health communication $(4.03 \pm 10.6 \mathrm{SD}$, range $0-5)$. Higher scores signify high knowledge, positive attitudes, or more health communication engagement about anal cancer. Most demonstrated high knowledge $(51 \%)$, and positive attitudes (90\%). Less than half $(47 \%)$ depicted engagement in health communication about anal cancer. There were no statistically significant differences were observed in knowledge, attitude, or health communication. HIV/HPV co-infection disproportionately predisposes PLWH to anal cancer. More efforts towards fostering anal cancerrelated knowledge, positive attitudes, and improving health communication behaviors among ASO employees, are needed. This would facilitate dissemination and promotion of anal cancer preventive measures i.e. HPV vaccine uptake and and anal anal cancer screening among PLWH. Objectives: Examine knowledge, attitudes, and health communication regarding HPV-associated anal cancer among nonclinical staff at HIV/AIDS service organizations (ASO), and compare responses between heterosexual females and heterosexual males; and LGBTQ+ females, and LGBTQ+ males. References: 1. Poon, M., Wong, J., Li, A., Manuba, M., Bisignano, A., Owino, M., \& Vahabi, M. (2018). HIV-positive MSM's knowledge of HPV and anal cancer self-sampling: A scoping review. Current oncology (Toronto, Ont.), 25(1), e83-e89. https://doi.org/10.3747/co.25.3856. 2. Wigfall, L. T., Bynum, S. A., Brandt, H. M., Sebastian, N., \& Ory, M. G. (2018). HPV-Related Cancer Prevention and Control Programs at CommunityBased HIV/AIDS Service Organizations: Implications for Future Engagement. Frontiers in oncology, 8, 422. https://oi.org/10.3389/ fonc. 2018.00422

\section{P58: Survival Disparities and BRCA Mutations in Breast Cancer Among Women of African Ancestry (WAA)}

\section{Yilin $\mathrm{Xu}$, Angelique Richardson, Georgia Robins Sadler UCSD Moores Cancer Center, La Jolla, CA, USA}

Abstract: Women of African Ancestry (WAA) have the highest breast cancer mortality rate. This literature review was conducted to gather the latest scientific evidence regarding correlations among BRCA mutations, triple-negative breast cancer (TNBC), and adverse outcomes in WAA to suggest hypothetical clinical care innovations worthy of scientific evaluation.Women of African Ancestry. This narrative literature review was undertaken as the first step in identifying the latest scientific discoveries related to these three clinical characteristics when present in WAA. It was intended to guide the development of an innovative, clinical practice hypothesis designed to be tested for its capacity to improve breast cancer survival outcomes for WAA. PubMed, ScienceDirect, and Google Scholar databases were explored using variations of such terms as breast cancer, Black, African, African-American, genetic mutations, BRCA 1, BRCA 2, TNBC, and health disparities. Accessible full-text articles written in English and published between 2005 and 2020 were eligible for review. In spite of the national focus to create better breast cancer-related outcomes, WAA continue to have the poorest survival rate. They are more likely to be diagnosed with the highly aggressive TNBC and at an earlier age. WAA were reported to have a higher percentage of BRCA 1 and 2 mutations. Evidence suggests that the presence of those BRCA mutations may be contributing to the disproportionate diagnosis of TNBC in WAA. Underuse of genetic testing for BRCA and other genetic mutations may put WAA at risk of delayed diagnosis and prompt treatment, thereby contributing to low survival rates. While studies identified the need for innovative clinical discoveries, a more immediate solution for reducing breast cancer mortality is the use of genetic screening. That will identify women at increased risk of mortality, facilitate monitoring, and provide the option of early intervention to increase their likelihood of survival. Objectives: The participant will be able to identify the correlation between BRCA mutations, rates of triple-negative breast cancer, and the survival disparities in women of African ancestry. The participants will learn about two steps that the community can take to address the breast cancer disparities and improve the survival outcome for WAA. References: Churpek J.E., Walsh T., Zheng Y., Moton Z., Thornton A.M., Lee M.K., Casadei S., Watts A., Neistadt B., Churpek M.M., et al. Inherited predisposition to breast cancer among African American women. Breast Cancer Res. Treat. 2015;149:31-39. doi: 10.1007/ s10549-014-3195-0. Newman, L. A. Disparities in Breast Cancer and African Ancestry: A Global Perspective. Breast J 21, 133-139 (2015). Yedjou, C. G. et al. Health and Racial Disparity in Breast Cancer. in Breast Cancer Metastasis and Drug Resistance (ed. Ahmad, A.) vol. 1152 31-49 (Springer International Publishing, 2019). 
P59: Sense of belonging in first-year and Asian cancer research doctoral students during a global pandemic

Misty Pocwierz-Gaines, Joyce Solheim

University of Nebraska Medical Center, Omaha, NE, USA

Abstract: The COVID-19 pandemic closed the University of Nebraska Medical Center campus and isolated graduate students. Small group Zoom meetings between first-year cancer research doctoral students, cancer research doctoral students of Chinese descent and a program administrator were held twice a month during this time with the intention of facilitating a stronger sense of belonging. Racism and decreased sense of belonging are two prominent issues for students. This closure and a rise in racism against individuals of Chinese descent caused significant emotional stress on students. These student populations were selected to meet in small groups via Zoom for informal conversations. The students of Chinese descent were in grouped together to facilitate conversations about racism. Students were asked about stress coping mechanisms that they were utilizing and if they had experienced racism. Success was measured by the willingness of students to continue participating and feedback provided two months after campus opened and students were able to resume full research activities. Each student that provided feedback reported that these meetings helped ameliorate feelings of isolation and confusion during the closure and encouraged them to feel as if they were valued by the program. All students felt that some version of this program should be continued for all first-year students, either in-person or online. Based on this outcome, a first-year program has been proposed for all future students. This model has potential to increase the sense of belonging of all students; this is especially important in the success of women and minority students. It can serve as a guide for institutions that desire to create a supportive environment with limited resources. Objectives: The participant shall be able to identify the role a welldeveloped sense of belonging plays in cancer research graduate students positive outcomes. The participant shall be able to identify at least one threat to cancer research graduate student success. The participant shall be able to identify at least one education intervention program that can be enacted with limited resources. References: Butts, Ga.C., Hurd, Y., Palermo, A.S., Delbrune, D., Saran, S., Zony, C., Krulwich, T. (2012) Role of institutional climate in fostering diversity in Biomedical research workforce: A case study. Mount Sinai Journal of Medicine. 79;498-511. Fisher, A.J., Mendoza-Denton, R., Patt, C., Young, I., Eppig, A., Garrell, R.L...Richards, M.A. (2019). Structure and belonging: Pathways to success for underrepresented minority and women $\mathrm{PhD}$ students in STEM fields. PloS ONE, 14(01), 1-14. doi: 10.1371/journal.pone.0209279. Museus, S.D., \& Park, J.J. (2015). The Continuing Significance of Racism in the Lives of Asian American College Students. Journal of College Student Development 56(6), 551-569. doi:10.1353/ csd.2015.0059.

\section{P60: Are cancer patients aware of the existence of carcinogenic food contaminants?}

Eden Augustus ${ }^{1}$, Isabella Francis-Granderson ${ }^{2}$

${ }^{I}$ The University of the West Indies, Cavehill Campus, Barbados, WI, Arima, Trinidad and Tobago, ${ }^{2}$ The University of the West Indies, St. Augustine Campus, Trinidad, W.I., St. Augustine, Trinidad and Tobago

Abstract: Diet is perceived to have a major role in cancer etiology. Programs assess and publicly report the human carcinogenicity of several substances; however, studies have highlighted that most persons find it difficult to differentiate fact from myth. Our study aims to examine the knowledge of carcinogenic food contaminants among cancer A series of 24-hour recalls, and questions based on the identification of carcinogens was administered to cancer patients $(n=40)$, by a trained interviewer. This sample was randomly selected as part of a ketogenic clinical trial. Participants were $>18$ years old, residents of Trinidad and Tobago, with no formal nutrition education or training. Consent was given by participants at the beginning of the study. Data was stored in an aggregated form and mainly described using univariate analysis. The study was approved by the research Ethics Committee, the Faculty of Medical Sciences, the University of the West Indies, St. Augustine Campus (CEC304/09/17) A variety of foods were identified by each patient. The most common foods included bread, rice, eggs, sausages, chicken, pork, salted fish, fish, cheese, juices (natural), sweetened juices and beverages, roti (dish made from flour), pasta,and pastries. The most common cooking methods were frying, roasting and barbeque / grilling, with less common methods of steaming and boiling. The majority of the participants $(n=38,95 \%)$ were aware that carcinogens are present in some foods and food substances. However when asked to identify any potential carcinogens from the recall, most participants $(72.5 \%)$, stated that there were none while $(27.5 \%)$ identified canned, salted and fried foods. This study aimed to examine food carcinogen knowledge. Our results revealed that although most of the sample were aware that carcinogenic food contaminants exist, most of the participants failed to identify foods within their recall that had potential carciogenic properties. Hence it's important to sensitise the public and support ongoing research. Objectives: 1 . The participant shall be able to identify the most common (typical) food consumed by a cohort of cancer patients diagnosed at least 6 months prior to the interview. 2. The participant shall be able to learn the awareness of the exisitance of food carcinogens among the cancer patient cohort. 3 . The particiapnt shall be able to access whether this cancer cohort was capable of naming the potential dood carcinogens. References: 1. Abnet CC. Carcinogenic Food Contaminants. Cancer Invest. 2007;25(3):189-96. 2. Carcinogens NRC (US) $\mathrm{C}$ on $\mathrm{CT}$ of NO. Introduction [Internet]. Carcinogens and Anticarcinogens in the Human Diet: A Comparison of Naturally Occurring and Synthetic Substances. National Academies Press (US); 1996 [cited 2020 Aug 9]. Available from: https://www.ncbi.nlm.nih. gov/books/NBK232619/. 3. (4) (PDF) Assessment of awareness towards some carcinogens in Hail region, Saudia Arabia [Internet]. ResearchGate. [cited 2020 Aug 9]. Available from: https://www.researchgate.net/publication/260001030_Assessment_of_awareness_towards_some_carcinogens_in_Hail_region_Saudia_Arabia

\section{P61: Will the Promise of the HPV Vaccine Work on Prevention of HPV Oral Cancer? Be Patient...}

\section{Sally Trinh, France Nguyen-Grozavu, Georgia Robins Sadler} UCSD Moores Cancer Center, La Jolla, CA, USA

Abstract: HPV Oral cancer is caused by a strain of the human papillomavirus (HPV). This cancer is predicted to surpass cervical cancer rates in 2020. This narrative review of the scientific literature explores what is known about the factors contributing to these increasing rates and how oral cancer can be reduced. This exploration of the peer-reviewed, evidence-based literature used variations of such terms as oral cancer, oropharyngeal cancer, human papillomavirus, HPV, risk, vaccine, prevention, incidence, epidemiology, race, trends, and sexual behavior. The databases used were PubMed, CINAHL, and PsycInfo. The inclusion criteria consisted of publications from 1983 to 2020 written in English. Publications that focused on multiple countries were included as long as there was data from the United States. Only full-text articles were eligible. The citations of the articles were used to search for other eligible articles. The biggest risk factor for HPV infection is unprotected oral sex, possibly due to changes in sexual behavior. This suggests that HPV oral cancer is a sexually-acquired disease. There is no standardized cancer screening for HPV oral cancer. Without a means to screen for HPV infection, it is difficult to control its spread. Thus, prevention of infection is heavily stressed in the literature through the HPV vaccine. Identified HPV vaccine clinical trials have shown promise in protecting against HPV16 infection. HPV16 is used as a marker because it is the main driver of HPV oral cancer. The HPV vaccine, approved by the FDA in 2006, is the only vaccine proven to prevent cervical cancer. It is possible that it may 
also prevent HPV oral cancer, but it will take until 2040 to determine whether the HPV vaccine fulfills the hope for reducing HPV oral cancer. Objectives: The participant shall be able to identify the limitations in the use of the HPV vaccine in preventing HPV oral cancer. The participant shall be able to identify another possible prevention method of HPV oral cancer. References: Timbang, M. R., Sim, M. W., Bewley, A. F., Farwell, D. G., Mantravadi, A., \& Moore, M. G. (2019). HPV-related oropharyngeal cancer: A review on burden of the disease and opportunities for prevention and early detection. Human Vaccines \& Immunotherapeutics, 15(7-8), 1920-1928. https://doi.org/10.1080/ 21645515.2019.1600985. Pinto, L. A., Kemp, T. J., Torres, B. N., Isaacs-Soriano, K., Ingles, D., Abrahamsen, M., Pan, Y., LazcanoPonce, E., Salmeron, J., \& Giuliano, A. R. (2016). Quadrivalent Human Papillomavirus (HPV) Vaccine Induces HPV-Specific Antibodies in the Oral Cavity: Results From the Mid-Adult Male Vaccine Trial. The Journal of infectious diseases, 214(8), 1276-1283. https://doi.org/10. 1093/infdis/jiw359

\section{P62: An Interviewer's Reflections of Interactions with Breast Cancer Research Participants: What every interviewer should know}

\section{Yvonne Cummings \\ Virginia Commonwealth University, Richmond, VA, USA}

\begin{abstract}
Patient interviewing is an important part of cancer research; yet more information seems to be available about recruiting participants than effectively interacting with them. This analysis examined the role of the interviewer when administering surveys to breast cancer patients and survivors. One female interviewer over 1000 interviews with breast cancer survivors over 15 years. Women newly diagnosed and undergoing primary treatment for breast cancer as well as women more than 12 months from diagnosis were interviewed as participants of various clinical trials. We discuss the bi-directional nature of the data collection process and describe how personal experiences with cancer shaped how this interviewer sharpened her skills to make each interview patient- focused. The more comfortable research Over time, this interviewer gained insight into multiple tools to aid interviewers in conducting timely and thorough interviews. We review key lessons and recommendations for interviewers of people with chronic illnesses. Research Interviewers should be able to: 1) thoughtfully prepare for the interaction with the participant, 2) be familiar with the survey measures, and 3) understand the importance of building rapport with participants. Positive participantinterviewer communication can impact research participant's willingness to enroll in future research studies. These three skills may enhance and interviews' ability to collect quality data and improve participant retention. Encouraging research interviewers to honor the process and sacrifice of the participant may enhance patient comfort when participating in research. Objectives: The participant should be able to identiy three methods for improving research interviews with participants. The author will explain how the personal experiences of the patient interviewer informed her future interactions with cancer survivors. Recommenations are made to other interviewers as to how to prepare for interviews, be familiar with research methods, and build rapport with study participants. References: DeJonckheere M, Vaughn LM. Fundamentals of semistructured interviews. Fam Med Com Health 2019;7:e000057.
\end{abstract}

P64: Does information seeking/avoiding impact prostate cancer treatment regret

Jala Lockhart ${ }^{1}$, Levi Ross ${ }^{1}$, Torhonda Lee ${ }^{2}$, Michael Preston ${ }^{3}$

${ }^{1}$ University of Alabama, Tuscaloosa, AL, USA, ${ }^{2}$ Florida A\&M University, Tallahassee, FL, USA, ${ }^{3}$ Virginia Commonwealth University, Richmond, VA, USA

Abstract: Theories of information seeking behavior recognize that information can be consciously sought out or avoided during treatment care planning. This research explores how prostate cancer $(\mathrm{PCa})$ patients interact with the treatment information environment and how information seeking/avoiding behaviors influence treatment regret. Face-to-face interviews were conducted with 63 men who were treated for or actively monitoring localized PCa. The survey collected demographic information, treatment information seeking/avoidance behavior, psychosocial characteristics, and perceptions of treatment regret. Information seeking/ avoidance behaviors were assessed by responses to $21 \mathrm{PCa}$ details representing 3 domains ( $1=$ treatment/care goals, $2=$ patient needs, and $3=$ social support). Response options ranged from " $1=$ Planned to look for this information" to " $4=$ Did not look for information and was not interested in learning anything about it." Bivariate comparisons were conducted using Pearson's chi-square tests for categorical variables. All statistical analyses were conducted using SPSS 21. Most participants were White $(58.7 \%)$, married $(79.4 \%)$, employed fulltime $(38.1 \%)$ or retired $(50.8 \%)$, completed high school $(96.7 \%)$, had private insurance $(50.7 \%)$, and had household incomes of $\$ 45,000$ or greater $(49.2 \%)$. Fewer than one-third of men planned to seek out comprehensive decision-making information in either domain during treatment care planning. Men were most interested in seeking out information in the treatment goals and patient needs domains, while planning to avoid social support information. There were no differences in self-reported treatment regret and information seeking/avoiding behaviors across the three domains. Even though men's information seeking/avoiding behaviors are not associated with treatment regret, cancer health educators should consider men's needs for information across multiple domains that address the full range of surviorship issues. Objectives: 1 . The participant shall be able to describe how $\mathrm{PCa}$ patients interact with the treatment information environment. 2. The participant shall be able to discuss/explain how information seeking/avoidance may influence treatment regret. References: Kassianos, A. P., Raats, M. M., \& Gage, H. (2016). An exploratory study on the information needs of prostate cancer patients and their partners. Health psychology research, 4(1). Loiselle, C. G. (2019). Cancer information-seeking preferences linked to distinct patient experiences and differential satisfaction with cancer care. Patient education and counseling, 102(6), 1187-1193.

P65: Universal Cancer Predisposition Screening Protocol in the Pediatric Oncology Population

Sapna Mehta ${ }^{1,2}$, Diane Masser-Frye ${ }^{2}$, Mehrzad Milburn² ${ }^{2}$ David Dimmock $^{2}$, Veronica Hoyo ${ }^{1}$, Dennis Kuo ${ }^{2}$

${ }^{1}$ University of California San Diego, San Diego, CA, USA, ${ }^{2}$ Rady Children's Hospital San Diego, San Diego, CA, USA

Abstract: Growing availability of gene sequencing has increased awareness of pediatric oncology cancer predisposition syndromes (CPS's). Studies indicate that about $8.5 \%$ of pediatric oncology patients have a CPS. This study investigates facilitators and barriers of the pediatric oncology CPS testing plan while providing the opportunity to receive genetic counseling and testing. This study surveys parents and adolescent patients before, during and after optional CPS testing and genetic counseling to analyze their knowledge, attitudes, and behaviors regarding CPS screening and survey their health literacy, demographic characteristics and cancer diagnosis. After Survey 1, family history is assessed with genetic counseling. The patients are offered genetic testing with the Invitae ${ }^{\mathrm{TM}}$ Multi-Cancer Panel and Survey 2 is administered. If opting to conduct testing and a pathogenic mutation exists, first-degree relative testing is offered. Finally, Survey 3 evaluates factors including satisfaction and expectations with CPS testing from the patient and parent experiences. The study has 60 patients enrolled with a goal of accruing 100 patients. From the 52 individuals who have received their genetic test results, $56 \%$ had negative results and $38 \%$ had variants of uncertain significance. $5 \%$ of the participants (two individuals) tested positive for likely pathogenic CPS mutations. This study includes a racially and socioeconomically diverse participant demographic. Higher health literacy 
is found to be linked with higher knowledge and awareness of CPS testing. There is positive parent and patient interest in conducting testing and receiving CPS knowledge, exemplified by how most patients opted to receive testing. Awareness of and interest in screening for CPS's is high in pediatric oncology patients and their parents. Medical providers need to strengthen their ability to educate and counsel patients about CPS testing, as they will face increasing demand for screening for CPS in pediatric oncology patients. Objectives: The participant shall be able to discuss general findings regarding the knowledge and attitudes of children with cancer and parents of these children. The participant shall be able to describe the facilitators and barriers involved in cancer predisposition testing in the pediatric oncology population. References: Zhang, J. et al. Germline Mutations in Predisposition Genes in Pediatric Cancer. N. Engl. J. Med. 373, 2336-2346 (2015). Phillips, K. A., Deverka, P. A., Hooker, G. W., \& Douglas, M. P. (2018). Genetic Test Availability And Spending: Where Are We Now? Where Are We Going?. Health affairs (Project Hope), 37(5), 710-716. Fecteau H, Vogel KJ, Hanson K, Morrill-Cornelius S. The evolution of cancer risk assessment in the era of next generation sequencing. J Genet Couns 2014;23:633-9. Schiffman, J. D. (2011). Hereditary cancer syndromes: If you look, you will find them. Pediatric Blood \& Cancer, 58(1), 5-6.

P66: Identifying Adjuvant Endocrine Therapy Group-based Trajectories: A Method to Target Interventions

Arnethea Sutton, Yangyang Deng, Vanessa Sheppard

Virginia Commonwealth University School of Medicine, Richmond, VA, USA

\begin{abstract}
Nonadherence to adjuvant endocrine therapy (AET) is suboptimal and complex particularly amongst subgroups of women. The purpose of this study was to identify clusters of individuals with similar adherence trajectories over time. Women with hormone receptor positive $(\mathrm{HR}+)$ breast cancer who initiated AET were recruited from three healthcare centers. Data were collected via surveys, medical and pharmacy records. The outcome, AET adherence, was captured from pharmacy
\end{abstract}

data and measured as the proportion of days covered (PDC) per year over 36 months; PDC was dichotomized at the 0.8 threshold. Covariates included demographic (e.g. race), psychosocial (e.g. emotional support), cancer care delivery (e.g. communication) factors, and AET-related symptoms. ANOVA tested differences between each trajectory group and symptom scores. Multivariable logistic regression models tested the effect from covariates on adherence. Most women were White $(70.8 \%)$, taking aromatase inhibitors $(58.7 \%)$, and had Stage I disease $(58.4 \%)$. Three trajectory groups emerged -1$)$ chronic nonadherence $(42.2 \%), 2$ ) suboptimal (40.4\%), and 3) high adherence (17.4\%). Adherence declined after year one for most women. In adjusted analysis, women in the chronic non-adherence group were almost 9 times more likely than women in the high adherence group to be unemployed (OR: 8.95; CI: 1.2 to 66.95 ). Women in the suboptimal group were more likely to have had less education (2.45; CI: 1.6 to 4.08$)$. Group-based trajectory analysis provides opportunities to identify subgroups most at risk for non-adherence and identify when non-adherence may occur. Social determinants appeared to more robust in distinguishing subgroups than other factors. Women who are unemployed may be in need of financial resources to promote AET adherence. Objectives: The participant shall be able to: 1) Describe group-based trajectories with regard to adherence. 2) Identify two social determinants that related to different trajectory patterns. References: Winn, Aaron N, \& Dusetzina, Stacie B. (2016). The association between trajectories of endocrine therapy adherence and mortality among women with breast cancer. Pharmacoepidemiology and Drug Safety, 25(8), 953959. Lambert-Côté, L., Bouhnik, A., Bendiane, M. et al. Adherence trajectories of adjuvant endocrine therapy in the five years after its initiation among women with non-metastatic breast cancer: a cohort study using administrative databases. Breast Cancer Res Treat180, 777-790 (2020). https://doi.org/10.1007/s10549-020-05549-x

Publisher's Note Springer Nature remains neutral with regard to jurisdictional claims in published maps and institutional affiliations. 